Western\&Graduate\&PostdoctoralStudies

Western University

Scholarship@Western

Electronic Thesis and Dissertation Repository

8-29-2016 12:00 AM

\title{
The neuroprotective effects of endurance training on the aging brain
}

Katelyn Norton, The University of Western Ontario

Supervisor: Kevin Shoemaker, The University of Western Ontario

A thesis submitted in partial fulfillment of the requirements for the Doctor of Philosophy degree in Kinesiology

(C) Katelyn Norton 2016

Follow this and additional works at: https://ir.lib.uwo.ca/etd

Part of the Cardiovascular System Commons, Exercise Physiology Commons, Exercise Science Commons, Other Neuroscience and Neurobiology Commons, Sports Sciences Commons, and the Systems and Integrative Physiology Commons

\section{Recommended Citation}

Norton, Katelyn, "The neuroprotective effects of endurance training on the aging brain" (2016). Electronic Thesis and Dissertation Repository. 4054.

https://ir.lib.uwo.ca/etd/4054

This Dissertation/Thesis is brought to you for free and open access by Scholarship@Western. It has been accepted for inclusion in Electronic Thesis and Dissertation Repository by an authorized administrator of Scholarship@Western. For more information, please contact wlswadmin@uwo.ca. 


\begin{abstract}
Dysregulation of autonomic control often develops with advancing age, favoring a chronic state of heightened sympathetic outflow with parasympathetic withdrawal. However, the mechanisms of this age-related autonomic impairment are not known and may relate to alterations in brain structure (e.g. cortical atrophy) and/or altered neural function, particularly in regions related to the cortical autonomic network, namely, the medial prefrontal cortex (MPFC), insula cortex (IC), and hippocampus (HC). Exercise exerts beneficial effects on brain structure and, in the case of cognition, neurologic function; however, how exercise affects regions of the brain related to autonomic function are not known. This thesis tested the hypothesis that changes in autonomic outflow across the adult age-span are related to cerebral cortex atrophy and function, and are sensitive to the effects of physical fitness. Study 1 demonstrated that advancing age impairs the heart rate (HR) response and modifies the cortical patterns associated with cardiovascular control during isometric handgrip (IHG), and is further exacerbated with coronary artery disease. The utility of aerobic exercise to prevent these age-related changes is not known. Study 2 revealed that lifelong, sustained aerobic training builds cortical reserve early in life, and sustains this benefit over the 40-70 year age span, but did not alter the rate of age-related cortical or subcortical decline. Study 3 demonstrated that cardiorespiratory fitness correlated strongly with whole-brain cortical thickness, while markers of autonomic outflow were specifically associated with cortical mass at the MPFC. Importantly, the strength of the relationship between autonomic variables and cortical thickness was determined by age, and was not altered following adjustments for cardiorespiratory fitness. Study 4 revealed a positive effect of high fitness on MPFC activation, yet did not affect absolute HR responses to IHG in this age range. Therefore, this series of studies implicates cortical atrophy in the frontal lobe as a contributor to the dysregulation of autonomic outflow associated with advancing age, and suggests that high cardiorespiratory fitness delays the age-related decline in cortical circuitry associated with cardiovascular control.
\end{abstract}




\section{Keywords}

cortical autonomic network, cardiorespiratory fitness, age, handgrip exercise, Masters athletes, cortical thickness, insula, medial prefrontal cortex 


\section{Co-Authorship Statement}

Katelyn N. Norton was the first author on the four papers that compose the body of this thesis and J. Kevin Shoemaker was the senior author. The co-authors on Chapter 2 were Mark B. Badrov, Carly C. Barron, Neville Suskin, and Armin Heinecke. The co-author on Chapter 3 was Robert Nikolov. The co-author for Chapter 4 was Torri A. Luchyshyn.

Specific contributions to the papers are listed as follows:

Conception and design: Katelyn N. Norton and J. Kevin Shoemaker

Data Collection: Katelyn N. Norton, Oksana Opalevych, Torri A. Luchyshyn, Mark B.

Badrov and Carly C. Barron.

Data Analysis and Interpretation: Katelyn N. Norton and J. Kevin Shoemaker.

Writing and Revisions: Katelyn N. Norton with revisions and feedback from all co-authors. 


\section{Acknowledgments}

I would like to acknowledge the technical assistance of Arlene Fleischhauer for performing phlebotomy and Oksana Opalevych for performing magnetic resonance imaging for all studies. I am also very grateful to Armin Heinecke for his helpful instruction and ongoing guidance regarding cortical data analysis. 


\section{Table of Contents}

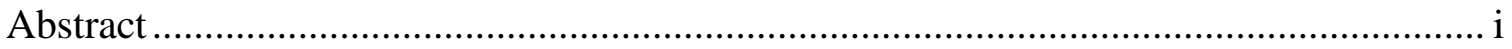

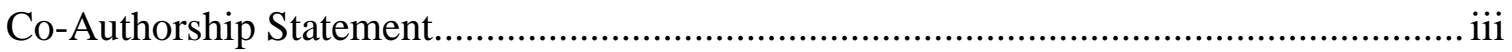

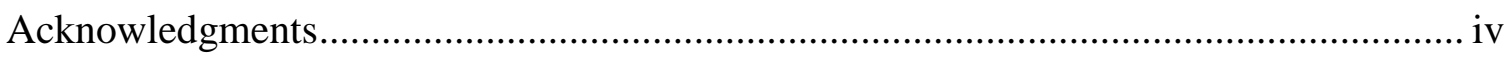

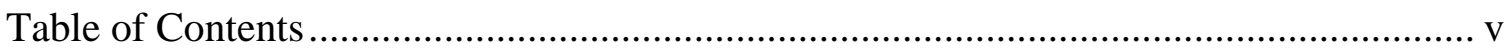

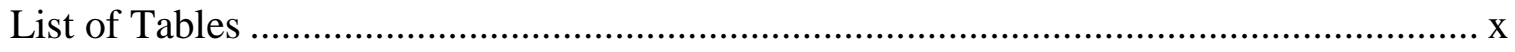

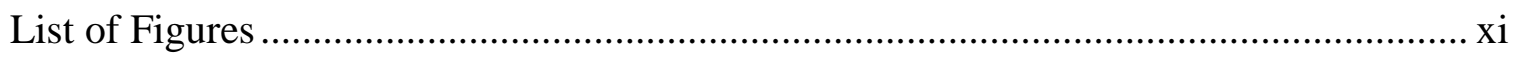

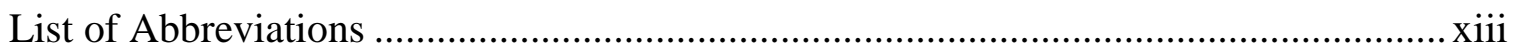

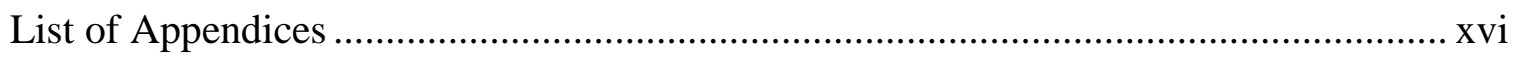

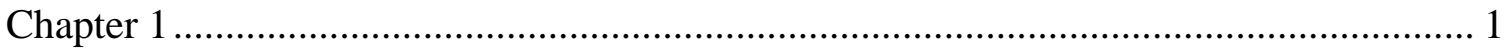

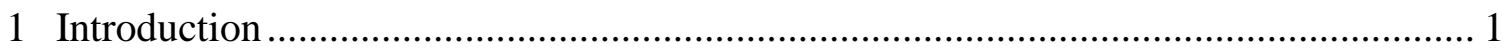

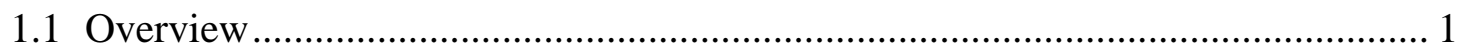

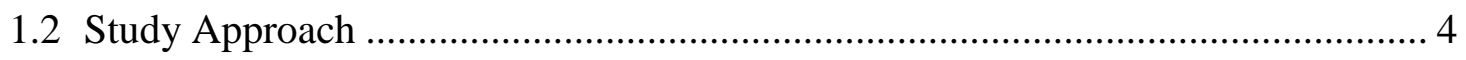

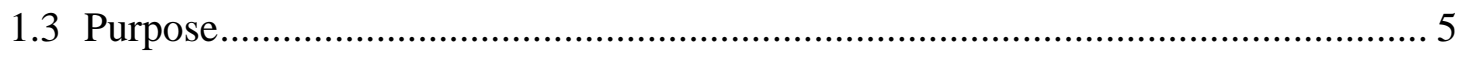

1.4 The Autonomic Nervous System .................................................................. 7

1.4.1 Sympathetic Nervous System ....................................................... 7

1.4.2 Parasympathetic Nervous System......................................................... 8

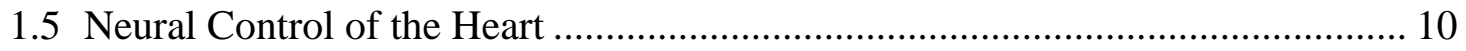

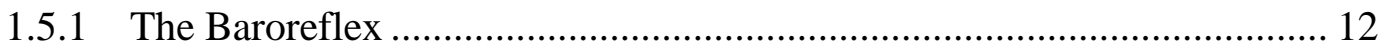

1.5.2 Heart Rate Variability ............................................................. 13

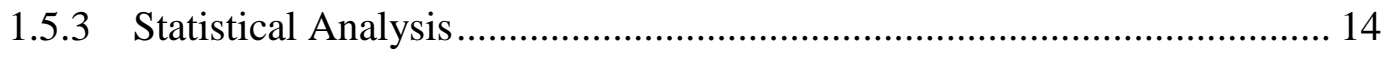

1.6 The Cortical Autonomic Network ................................................................ 15

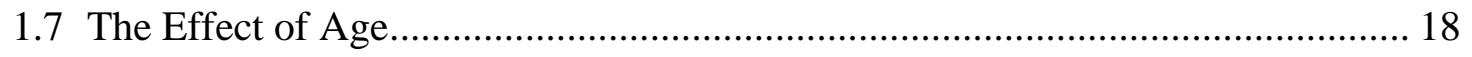

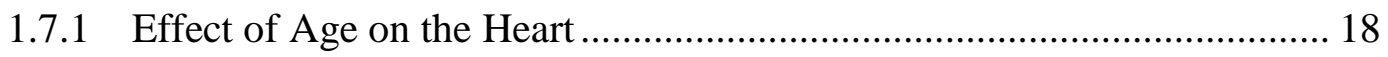

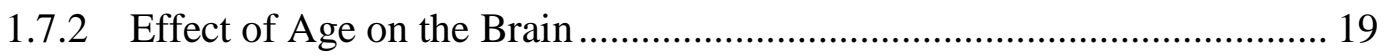




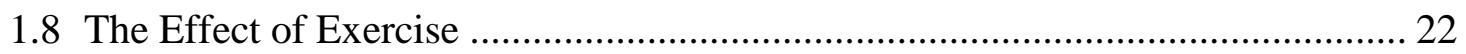

1.8.1 Effect of Acute Exercise on the Heart ..................................................... 22

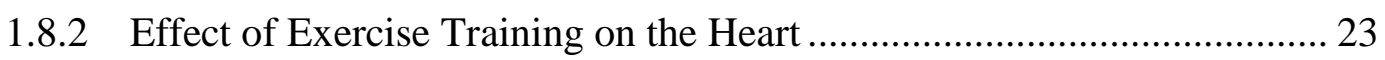

1.8.3 Effect of Exercise on the Brain ............................................................ 25

1.9 Determinants of Cortical Thickness and Brain Function..................................... 28

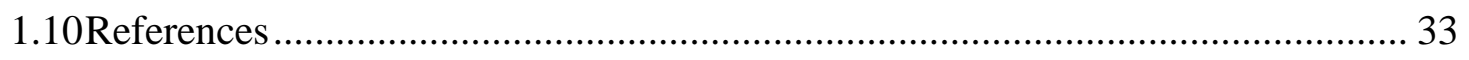

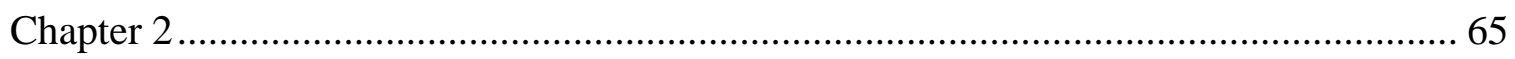

2 Coronary Artery Disease Affects Cortical Circuitry Associated with Brain-Heart

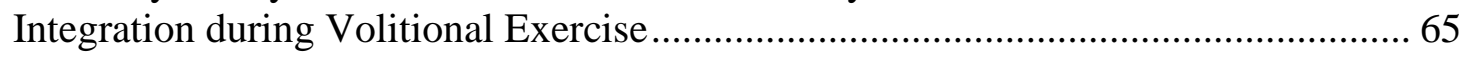

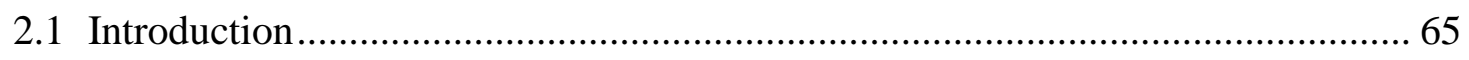

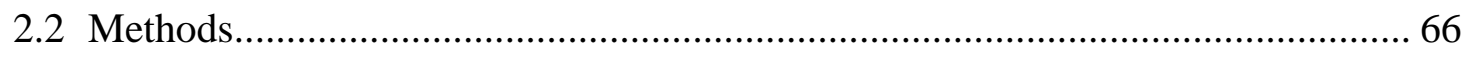

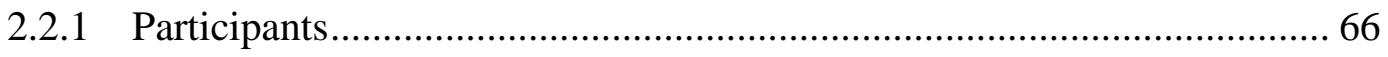

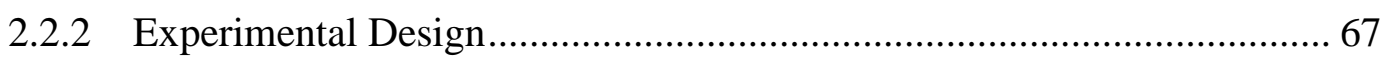

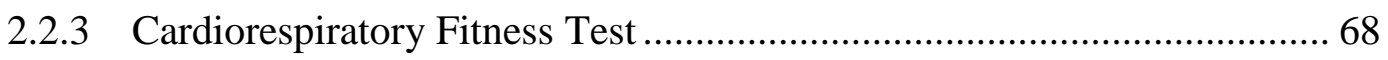

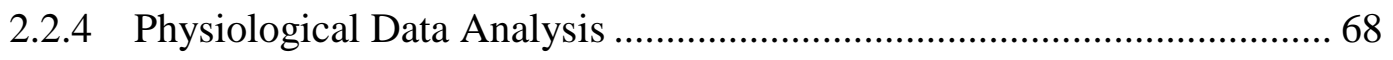

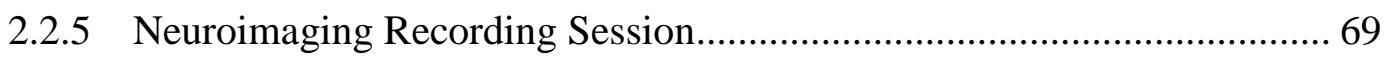

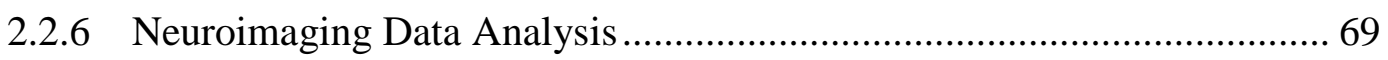

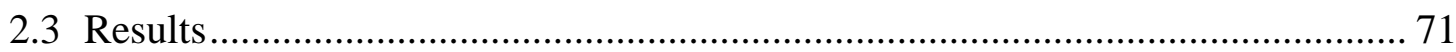

2.3.1 Physiological Responses .................................................................... 71

2.3.2 Functional (BOLD) Imaging Data: First-level (Individual) Response to 40\% IHG Task ................................................................................... 71

2.3.3 Functional (BOLD) Imaging Data: First-level (Individual) Response Correlated with Heart Rate ..................................................................... 72

2.3.4 Functional (BOLD) Imaging Data: Second-level (Group) Response to 40\% IHG Task ……........................................................................... 72

2.3.5 Contrasting BOLD Responses between Control and CAD to 40\% IHG

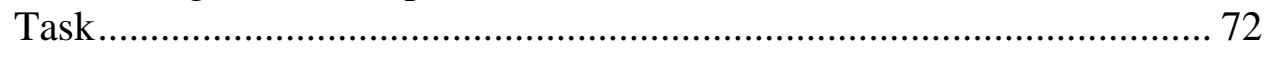

2.3.6 Functional (BOLD) Imaging Data: Second-level (Group) Response Correlated with Heart Rate 
2.3.7 Contrasting BOLD Responses between Control and CAD Correlated with Heart Rate …….............................................................................. 73

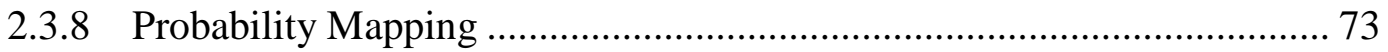

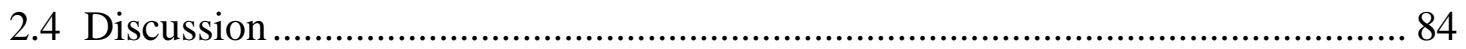

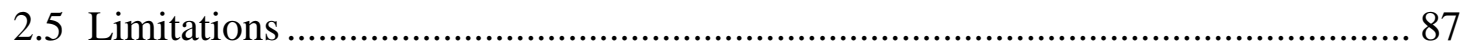

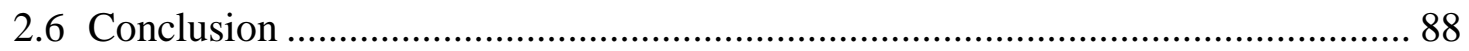

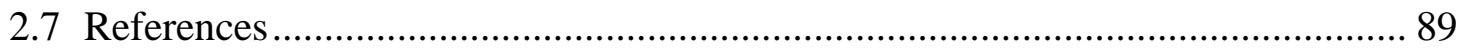

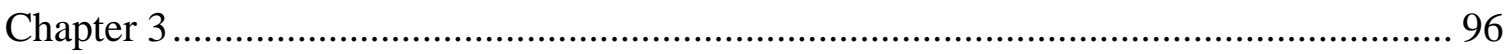

3 Impact of Long-Term Endurance Training Versus Guideline-Based Physical Activity on Brain Structure in Healthy Aging ..................................................................... 96

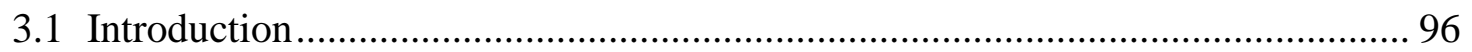

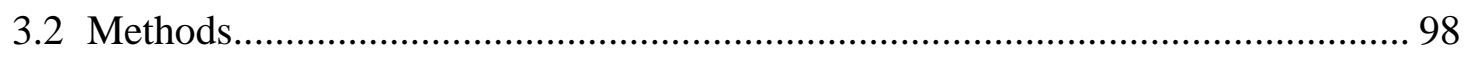

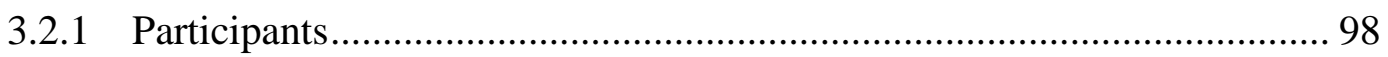

3.2.2 Assessment of Cardiorespiratory Fitness ................................................ 99

3.2.3 Physiological Data Acquisition................................................................ 99

3.2.4 Neuroimaging Data Acquisition ........................................................... 100

3.2.5 Neuroimaging Data Analysis ............................................................... 100

3.2.6 Statistical Analysis ............................................................................. 102

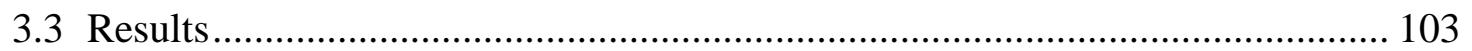

3.3.1 Subcortical Gray Matter.......................................................................... 103

3.3.2 Cortical Gray Matter ............................................................................ 104

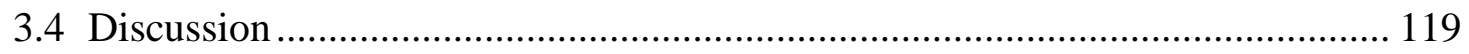

3.4.1 Subcortical Gray Matter and Lateral Ventricular Volume ..................... 120

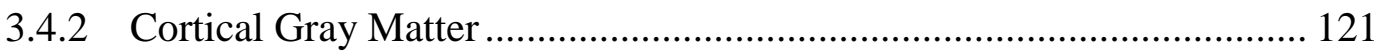

3.4.3 Negative Association with High Fitness and Brain Structure ................ 122

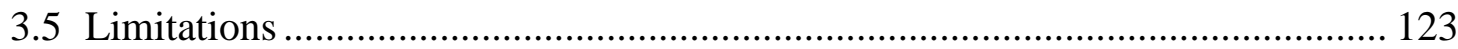

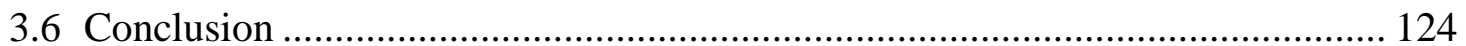




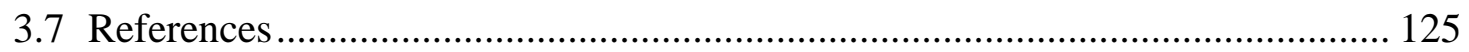

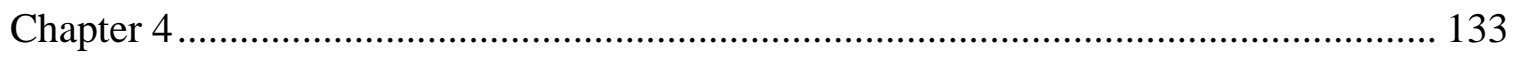

4 Cerebral Cortical Thickness Correlates with Autonomic Outflow ........................... 133

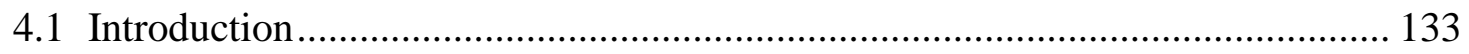

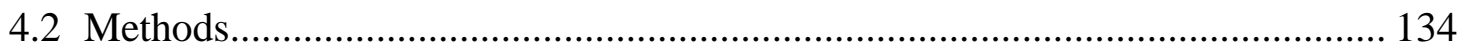

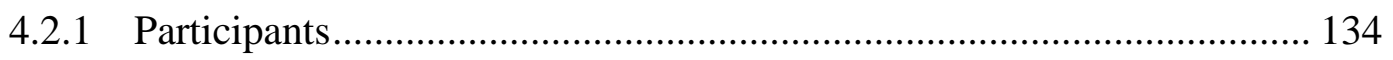

4.2.2 Assessment of Cardiorespiratory Fitness ................................................. 135

4.2.3 Physiological Data Acquisition............................................................. 135

4.2.4 Cardiovagal Baroreflex Sensitivity ...................................................... 135

4.2.5 Heart Rate Variability ....................................................................... 136

4.2.6 Sympathetic Neural Recordings …………......................................... 136

4.2.7 Neuroimaging Data Acquisition ………………..................................... 136

4.2.8 Neuroimaging Data Analysis .................................................................. 137

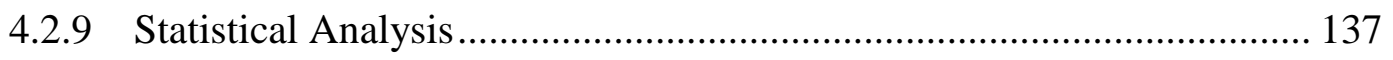

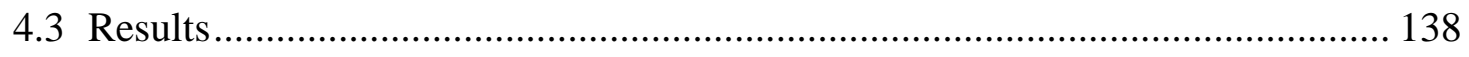

4.3.1 Whole-Brain Regression Results ...................................................... 138

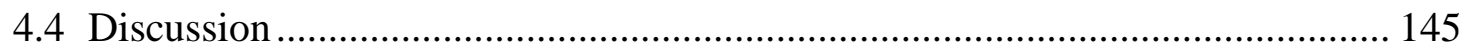

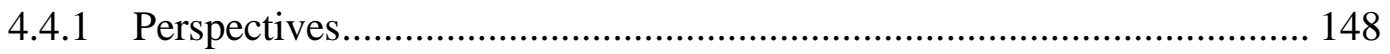

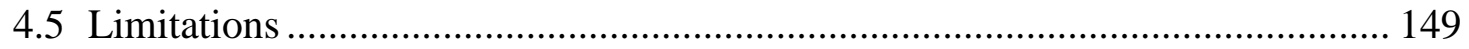

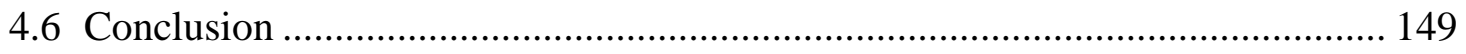

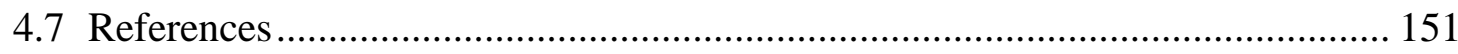

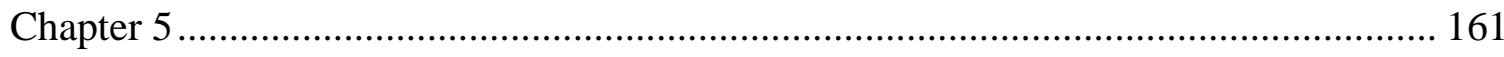

5 High Cardiorespiratory Fitness in Middle-Age Preserves the Cortical Circuitry Associated with Brain-Heart Integration During Volitional Exercise ........................ 161

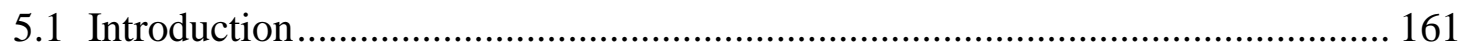

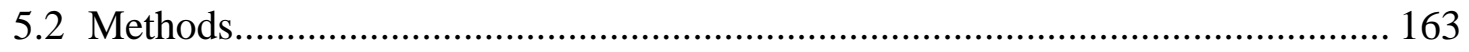




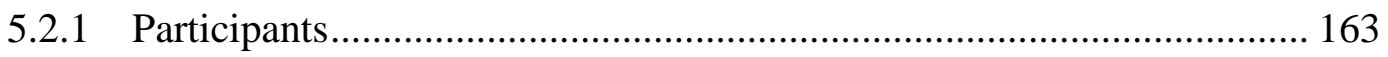

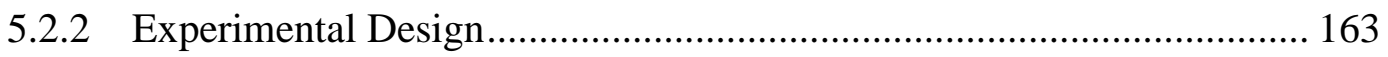

5.2.3 Assessment of Cardiorespiratory Fitness .............................................. 164

5.2.4 Physiological Data Acquisition................................................................ 164

5.2.5 Physiological Data Analysis ................................................................ 165

5.2.6 Neuroimaging Recording Session.......................................................... 165

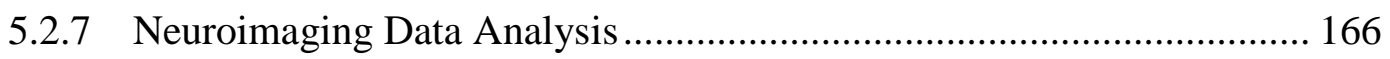

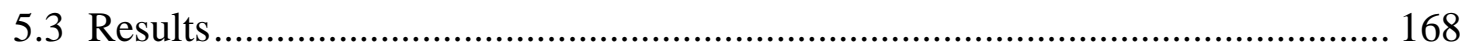

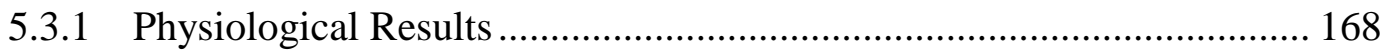

5.3.2 Functional (BOLD) Imaging Results: Handgrip Stimulus ...................... 168

5.3.3 Functional (BOLD) Imaging Results: Heart Rate .................................. 169

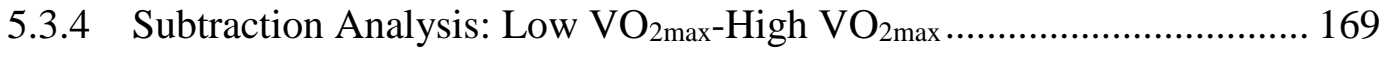

5.3.5 Psychophysiological Interaction Analysis: Heart Rate Time Course ..... 169

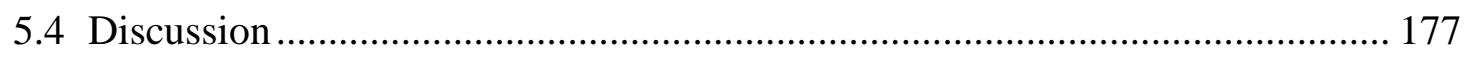

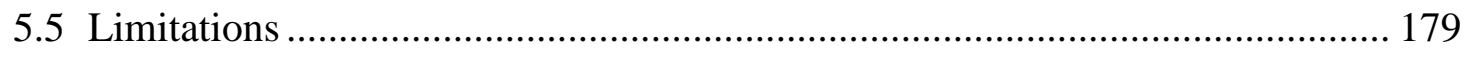

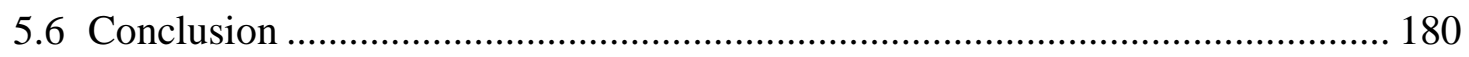

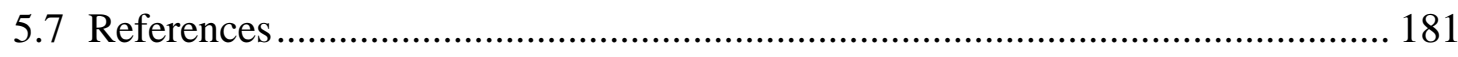

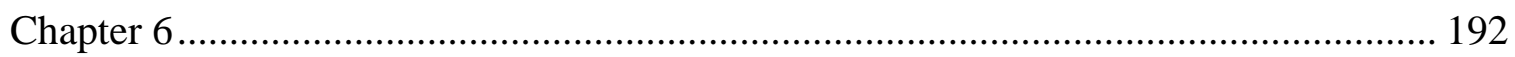

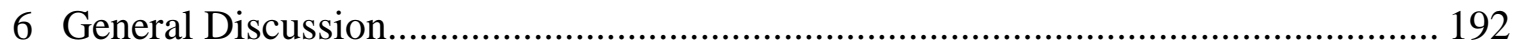

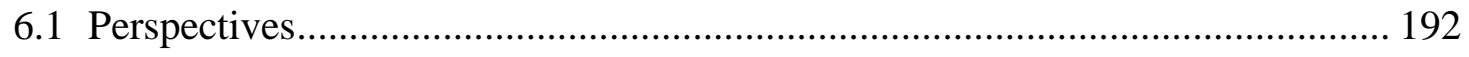

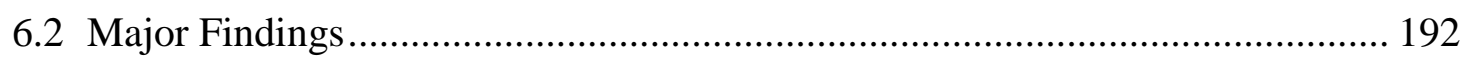

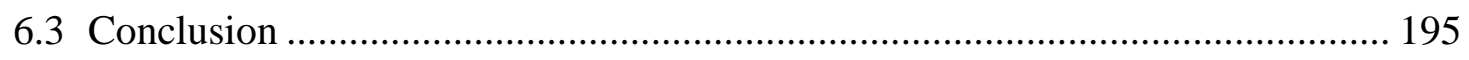

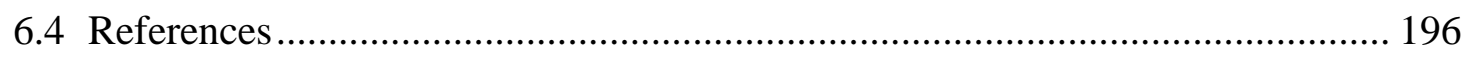

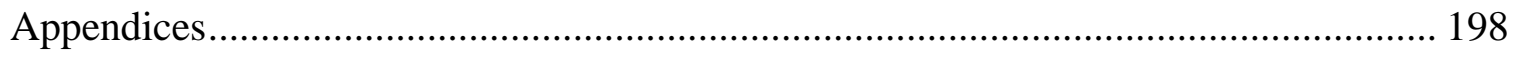

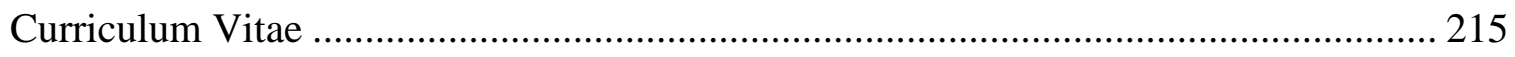




\section{List of Tables}

Table 2.1 Anthropometric and baseline cardiovascular data during baseline and isometric

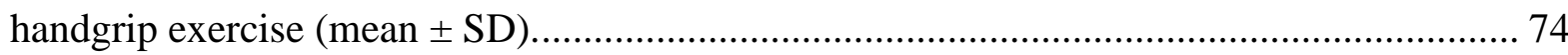

Table 2.2 BOLD signal changes to 40\% MVC handgrip in Control subjects...................... 75

Table 2.3 BOLD signal changes to 40\% MVC handgrip in CAD subjects........................ 76

Table 3.1 Anthropometric and baseline hemodynamic data (mean \pm SD). ...................... 106

Table 3.2 Whole-brain subcortical volumes.......................................................... 107

Table 3.3 Results of multiple linear regression of ROI subcortical volumes against

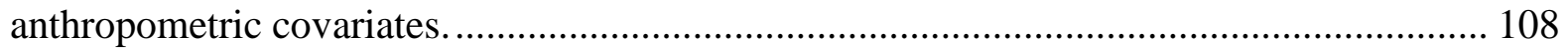

Table 3.4 Right hemisphere brain regions showing significant group differences in cortical

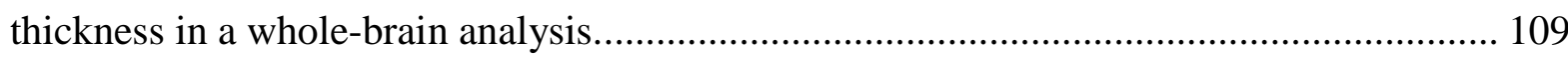

Table 3.5 Left hemisphere brain regions showing significant group differences in cortical

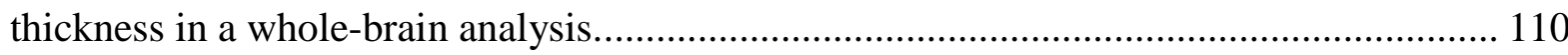

Table 3.6 Results of multiple linear regression of ROI cortical thickness against anthropometric covariates

Table 4.1 Anthropometric and baseline autonomic data (mean \pm SD)........................... 140

Table 4.2 Linear regression of autonomic variables on cortical thickness. ...................... 141

Table 4.3 Results of multiple linear regression based on simple linear regression............. 142

Table 5.1 Anthropometric and baseline cardiovascular data (mean \pm SD). ..................... 170

Table 6.1 Cortical and physiological results from all studies representing a continuum of risk

for advancing age and reduced fitness with associated autonomic outcomes. 197 


\section{List of Figures}

Figure 2.1 Graded heart rate response to isometric handgrip.

77

Figure 2.2 Cortical functional response to $40 \%$ IHG task in Control (CTRL; top three images) and CAD (lower three images)

Figure 2.3 Subtraction Result for Group 1 average (CAD) vs Group 2 average (Control) to $40 \%$ IHG boxcar analysis

Figure 2.4 Cortical functional response correlated to individual heart rate time course during $40 \%$ IHG task in Control (CTRL; top three images) and CAD (lower three images).

Figure 2.5 Subtraction result for Group 1 average (CAD) vs Group 2 average (Control) correlated to individual heart rate time course during $40 \%$ IHG task. 81

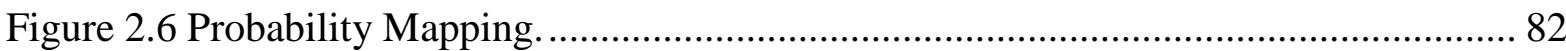

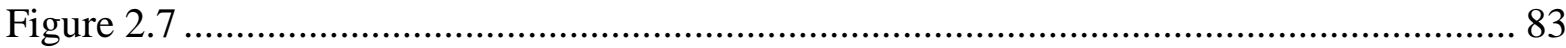

Figure 3.1 Regression analysis of region-of-interest subcortical volumes with age. .......... 112

Figure 3.2 Group differences in cortical thickness (MA > HA) ...................................... 113

Figure 3.3 Regression analysis of region-of-interest cortical thickness with age................ 114

Figure 3.4 Full cortex correlation analysis with age covariate. ..................................... 115

Figure 3.5 Full cortex negative correlation analysis with $\mathrm{VO}_{2 \max }$ covariate. .................... 116

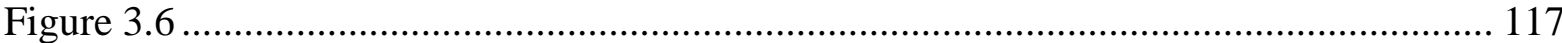

Figure 3.7 Full cortex correlation analysis with $\mathrm{VO}_{2 \max }$ covariate.................................. 118

Figure 4.1 Whole-brain correlation of selected autonomic variables and cortical thickness. 
Figure 4.2 Regression analysis of significant autonomic variables with cortical thickness at the left medial prefrontal cortex.

Figure 5.1 Average time course of the heart rate response for the isometric handgrip exercise trials of the neuroimaging session 171

Figure 5.2 Heart Rate (HR) response to isometric handgrip regressed with cardiorespiratory

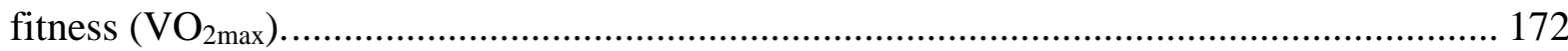

Figure 5.3 BOLD Imaging Results: Handgrip Stimulus............................................. 173

Figure 5.4 BOLD Imaging Results: Heart Rate Regressor.......................................... 174

Figure 5.5 Subtraction analysis of $\mathrm{Low} \mathrm{VO}_{2}-\mathrm{High} \mathrm{VO}_{2}$ representing the difference in activation patterns that correlate with heart rate. 175

Figure 5.6 PPI Results: Functional connectivity of the MPFC during handgrip stimulus regressed with HR time course. 


\section{List of Abbreviations}

\begin{tabular}{|c|c|}
\hline $\mathrm{ACC}$ & Anterior Cingulate Cortex \\
\hline $\mathrm{ACh}$ & Acetylcholine \\
\hline $\mathrm{AChE}$ & Acetylcholinesterase \\
\hline ANS & Autonomic Nervous System \\
\hline AV & Atrioventricular \\
\hline BDNF & Brain-derived neurotrophic factor \\
\hline BMI & Body Mass Index \\
\hline BOLD & Blood-oxygen-level-dependent \\
\hline BP & Blood Pressure \\
\hline BRS & Baroreflex Sensitivity \\
\hline CAD & Coronary Artery Disease \\
\hline $\mathrm{CO}$ & Cardiac Output \\
\hline DBP & Diastolic Blood Pressure \\
\hline ECG & Electrocardiogram \\
\hline fMRI & Functional Magnetic Resonance Imaging \\
\hline FDR & False Discovery Rate \\
\hline HA & Healthy Active \\
\hline $\mathrm{HC}$ & Hippocampus \\
\hline HR & Heart Rate \\
\hline
\end{tabular}




\begin{tabular}{|c|c|}
\hline HRV & Heart Rate Variability \\
\hline IC & Insula Cortex \\
\hline IGF-1 & Insulin-like Growth Factor 1 \\
\hline IHG & Isometric Handgrip \\
\hline $\mathrm{K}$ & Potassium \\
\hline LVEF & Left-ventricular Ejection Fraction \\
\hline MA & Master's Athletes \\
\hline MAP & Mean Arterial Pressure \\
\hline MPFC & Medial Prefrontal Cortex \\
\hline MSNA & Muscle Sympathetic Nerve Activity \\
\hline MVC & Maximal Voluntary Contraction \\
\hline NTS & Nucleus Tractus Solitarus \\
\hline PCC & Posterior Cingulate Cortex \\
\hline PNS & Parasympathetic Nervous System \\
\hline PPI & Psychophysiological Interaction \\
\hline RFX & Random Effects \\
\hline ROI & Region-of-Interest \\
\hline RVLM & Rostral Ventrolateral Medulla \\
\hline SA & Sinoatrial \\
\hline SBP & Systolic Blood Pressure \\
\hline
\end{tabular}


SDNN

SNS

VEGF

$\mathrm{VO}_{2 \max }$
Standard Deviation of Normal-to-Normal RR Intervals

Sympathetic Nervous System

Vascular Endothelial-derived Growth Factor

Maximal Oxygen Consumption 


\section{List of Appendices}

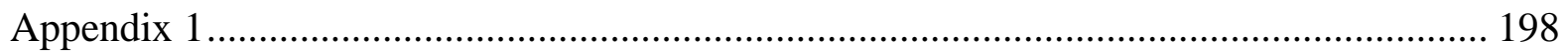

Appendix 2 Ethics Approval................................................................................... 214 


\section{Chapter 1}

\section{Introduction}

\subsection{Overview}

As a major risk factor for dementia and other neurologic impairments, the current trajectory of our aging population will produce an unsustainable health and economic burden for future generations. Advancing age is often associated with cortical atrophy (Raz, Lindenberger et al. 2005), changes in brain functional responses (Nyberg, Salami et al. 2010), and declines in cognitive performance (Ronnlund, Nyberg et al. 2005). Autonomic dysregulation represents a further insidious age-related neurological impairment that threatens morbidity and mortality (Nicolini, Ciulla et al. 2014) through detrimental effects on blood pressure control (Izzo, Smith et al. 1987, Izzo and Taylor 1999), exacerbation of atherosclerosis (Zhang and Faber 2001), cardiac tissue damage, and heightened risk of arrhythmias (Hachinski, Wilson et al. 1992). Nonetheless, substantial inter-individual differences exist with some individuals showing resistance to major age-related brain pathologies (Nyberg, Lovden et al. 2012, Pudas, Persson et al. 2013). The large heterogeneity in brain aging represents a major scientific challenge that has led researchers to examine what factors contribute to this protection against imminent age-related decline. In the last decade, it has frequently been suggested that physical activity may have positive global influences on brain health, including spared brain volume (Erickson, Prakash et al. 2009, Erickson, Voss et al. 2011, Niemann, Godde et al. 2014), improved task-related functional responses (Colcombe, Kramer et al. 2004, Voelcker-Rehage, Godde et al. 2010), increased white matter integrity (Johnson, Kim et al. 2012, Voss, Heo et al. 2013), and cognitive performance (Josefsson, de Luna et al. 2012). However, whether exercise has a similar impact on autonomic function has only recently been explored (Carter, Banister et al. 2003) showing the most benefit in aged individuals or those with pathological conditions that raise baseline sympathetic outflow, such as heart disease. Further, existing data are difficult to interpret in terms of the overall exercise benefit. Prospective training studies normally are of short duration $(<12$ months) and are employed in a narrow age range of senescent individuals with a starting 
point of cognitive impairment or cardiovascular disease. Therefore, there is need to understand the importance of intervention timing and the exercise dose response patterns across the adult age range during which brain atrophy occurs.

Coronary artery disease (CAD) increases the risk for stroke, cognitive impairment and autonomic dysregulation (Martins, Hone et al. 2006, Zulli, Nicosia et al. 2008, Roberts, Knopman et al. 2010, Barekatain, Askarpour et al. 2014). Impaired autonomic outcomes of $\mathrm{CAD}$ may exacerbate the disease pattern though tissue damage and/or a diminished ability to affect rapid adjustments in response to stress and, thereby, limit the benefits that can be derived from exercise rehabilitation. Moreover, our laboratory previously reported accelerated age-related cortical atrophy in CAD patients (Anazodo, Shoemaker et al. 2013). However, data are limited regarding the impact of CAD on the brain-heart connection and the utility of aerobic exercise to prevent these insidious changes.

Experimental models in rodents (Cechetto and Chen 1990, Dampney 1994, Verberne 1996), as well as clinical (Norris, Froggatt et al. 1978, Critchley, Mathias et al. 2003, Woo, Macey et al. 2003, Soros and Hachinski 2012, Woo, Yadav et al. 2014), and observational studies in humans (Critchley, Corfield et al. 2000, Cechetto and Shoemaker 2009, Thayer, Sollers et al. 2009, Shoemaker and Goswami 2015), indicate that several cortical sites modulate autonomic cardiovascular control. In fact, cognitive (Critchley, Mathias et al. 2003, Critchley, Wiens et al. 2004, Critchley, Rotshtein et al. 2005, Critchley, Nagai et al. 2011) and autonomic network regions (Cechetto and Shoemaker 2009, Shoemaker and Goswami 2015) appear to overlap in critical cortical sites including the medial prefrontal cortex (MPFC), hippocampus (HC), anterior cingulate cortex (ACC), and insula cortex (IC) (Barron and Chokroverty 1993, Soufer, Bremner et al. 1998, Critchley, Corfield et al. 2000, Gianaros, Van Der Veen et al. 2004, Gianaros, Derbyshire et al. 2005). The prefrontal areas of the brain, which are the last to fully mature in the early twenties, are the first to show signs of decline as early as age forty (Raz, Lindenberger et al. 2005) with degradation of fiber myelination (Northoff, Richter et al. 2000), and a loss of structural connectivity (O'Sullivan, Jones et al. 2001). Significant but more moderate declines are seen in the temporal, parietal and occipital cortices, respectively (Raz and Rodrigue 2006). In addition, the hippocampus, amygdala 
and cerebellum all show signs of age-related atrophy (Raz and Rodrigue 2006). Consequently, alterations in cerebral cortex structure may lead to generalized impairment across several neurological outcomes. Thus, prevention or treatment of cortical degradation with age may yield significant social and clinical outcomes. 


\subsection{Study Approach}

Moderate intensity isometric handgrip ( $\mathrm{IHG}$ ) exercise of short duration produces a rapid tachycardia in young and healthy individuals (Mancia, Iannos et al. 1978, Mark, Victor et al. 1985, Wong, Masse et al. 2007), offering a unique opportunity to explore the cortical representation of autonomic cardiac control. Pharmacologic evidence indicates that a decrease in parasympathetic dominance accounts for much of this rapid heart rate (HR) change (Hollander and Bouman 1975, Fagraeus and Linnarsson 1976, Mitchell, Reeves et al. 1989). In young individuals, the magnitude of this rapid increase in HR with IHG exercise is correlated with reduced activity within the MPFC (Gianaros, Van Der Veen et al. 2004, Wong, Masse et al. 2007) and the HC (Norton, Luchyshyn et al. 2013). Age and coronary artery disease (CAD) are associated with impaired autonomic outcomes including diminished parasympathetic modulation of HR (Mancia, Cleroux et al. 1991, Seals, Taylor et al. 1994, Ford 1999). It follows that these regions are among those associated with cardiovagal control and form our a priori cortical regions of interest including the IC and ACC.

T1-weighted structural acquisition at 3-Tesla will enable quantification of cortical thickness and subcortical gray and white matter volumes, while T2-weighted functional neuroimaging will examine blood-oxygen-level-dependent (BOLD) activation patterns in response to the IHG task.

Age, cardiorespiratory fitness, and markers of autonomic outflow will be treated as possible covariates. 


\subsection{Purpose}

The purpose of this study was to test the interaction between cardiorespiratory fitness and cortical structure and functional responses related to the neural control of the circulation. The working hypothesis is that differences in autonomic outflow across the adult agespan are related to cerebral cortex atrophy and function, and are sensitive to the effects of physical fitness. We suggest that brain structure and functional responses are modified by negative (pathology) and positive (cardiorespiratory fitness) behaviors and that such impairment, or improvement, is associated with HR control, particularly emphasizing activity patterns within discrete regions of the cortical autonomic network, namely the MPFC, IC, and HC.

\section{Study 1. Coronary artery disease affects cortical circuitry associated with brain- heart integration during volitional exercise.}

Purpose: To determine if CAD negatively impacts the cortical and HR response to volitional IHG.

Hypothesis: Coronary artery disease is associated with a reduced HR response to volitional exercise, which is accompanied by dysregulation of the cortical autonomic network.

\section{Study 2. Impact of long-term endurance training vs. guideline-based physical activity on brain structure in healthy aging.}

Purpose: To determine the maximal benefit of exercise training on cortical and subcortical gray matter.

Hypothesis: Maximal levels of aerobic exercise training will provide benefits to brain structure beyond those achieved through guideline-based fitness.

\section{Study 3. Regional cerebral cortical thickness correlates with autonomic outflow.}

Purpose: To examine the relationship between cortical structure and autonomic outflow in aging adults. 
Hypothesis: Cortical atrophy predicts age-related changes in autonomic outflow.

Study 4. High cardiorespiratory fitness in middle-age preserves the cortical circuitry associated with brain-heart integration during volitional exercise.

Purpose: To examine how cardiorespiratory fitness affects the cortical circuitry associated with cardiovascular arousal.

Hypothesis: High cardiorespiratory fitness prevents the age-related decline in cortical circuitry associated with isometric handgrip and cardiovascular arousal. 


\subsection{The Autonomic Nervous System}

The autonomic nervous system (ANS) is responsible for virtually every aspect of involuntary physiologic control. It is structurally and functionally positioned to interface between the internal and external environments, coordinating bodily functions to ensure homeostasis, and generate adaptive responses. Thus, the ANS has the crucial task of overseeing system regularity and ultimately ensuring our survival. The ANS is comprised of three divisions: the sympathetic and parasympathetic nervous systems, as well as the enteric nervous system. It is through the sympathetic and parasympathetic centrallyorchestrated neural systems that the body achieves regulation over the cardiovascular system. A dynamic balance occurs between these two effectors such that the development and control of cardiovascular regulation at any given level depends upon the balance between their respective activities or dominance of functional outcome.

\subsubsection{Sympathetic Nervous System}

Pre- and post-ganglionic adrenergic axons ensure transmission of signals through the sympathetic nervous system (SNS), which work to mobilize the body's resources for action under stress. The shorter preganglionic neurons of the SNS originate in the interomedial cell column of the thoracic and lumbar spinal cord (T1 - L2), and receive input signals from multiple transmitter systems in the hypothalamus, brainstem, and autonomic control centers within the cortex (Shields 1993). Axons from the preganglionic neurons project to a chain of ganglia located near the spinal cord (collectively referred to as the sympathetic trunk) where they synapse with much longer postganglionic neurons. At these synapses, preganglionic neurons release acetylcholine $(\mathrm{ACh})$, a neurotransmitter that binds to nicotinic cholinergic receptors on postganglionic dendrites. Upon stimulation of postganglionic neurons, action potentials descend along their axons to peripheral targets where various neurotransmitters are released, thereby activating receptors on the peripheral tissues. Targets of the SNS include the vascular smooth muscle, myocardial and pacemaker cells of the heart, as well as smooth muscle cells surrounding the vasculature in skeletal muscle, visceral organs, and skin (Shields 1993). It is the activation of these target tissue receptors that causes end-organ sympathetic effects on the blood vessels and the heart. 
Within the blood vessels, small enlargements along the nerve fibers, called varicosities, are the site of neurotransmitter storage and release. There are several neurotransmitters released from sympathetic nerve terminals during sympathetic discharge. Norepinephrine, neuropeptide $\mathrm{Y}$ and adenosine triphosphate all exert effects on vascular smooth muscle when they bind to their corresponding receptors. Generally, the overall effect of sympathetic innervation on the vascular system is vasoconstriction causing an increase in vascular resistance and cardiac output, and a decrease in peripheral blood flow (Robertson, Waller et al. 1988).

Direct recordings of sympathetic outflow in humans are made with the use of microneurographic techniques (Hagbarth and Vallbo 1968) from the populations of postganglionic sympathetic neurons innervating the skeletal muscle vasculature (muscle sympathetic nerve activity; MSNA). The measured bursts of activity reflect periods of efferent action potential synchronization (Delius, Hagbarth et al. 1972). The size of any given burst is determined by the proximity of the recording electrode to the individual neurons within the respective bundle of sympathetic axons (Tompkins, Melling et al. 2013), as well as the number (Ninomiya, Malpas et al. 1993) and size (Salmanpour, Brown et al. 2011) of action potentials.

Sympathetic activation of the heart through the cardiac nerves results in tachycardia and increased contractility. Neural activation of the sinoatrial (SA) and atrioventricular (AV) nodes acts to increase HR. When the SA node receives sympathetic stimulation, norepinephrine is released from the nerve endings and binds to adrenergic receptors on the pacemaker cell membrane. Innervation of the myocardial fibers causes an increase in the force of contraction at the atria and ventricles. As a result of these combined effects, enhanced sympathetic activity causes blood pressure and HR to rise and is particularly important during periods of excitement and stress.

\subsubsection{Parasympathetic Nervous System}

Similar to the SNS, the parasympathetic nervous system (PNS) follows a two-neuron efferent system that has both preganglionic and postganglionic neurons. The preganglionic fibers of the PNS originate from the craniosacral regions of the spinal cord, 
including the medial medullary sites of the nucleus ambiguus, nucleus tractus solitarius, and dorsal motor nucleus (Olshansky, Sabbah et al. 2008). In the medulla, the cranial nerves III (oculomotor nerve), VII (facial nerve), IX (glossopharyngeal nerve), and X (vagus nerve) form the preganglionic parasympathetic fibers (Guyenet 2006), which project to ganglia very close to their visceral targets. The most important cranial nerve responsible for cardiovascular control is the vagus nerve, containing nearly $90 \%$ of the preganglionic parasympathetic fibers in the body (Marieb E 2004). As indicated in the SNS, preganglionic innervation in the PNS is cholinergic with these terminals releasing $\mathrm{ACh}$ at the ganglion synapse onto nicotinic receptors.

The parasympathetic influence on cardiac function occurs predominantly through the binding of ACh to muscarinic receptors in the heart. There are three main types of muscarinic receptors that are well characterized: $M_{1}, M_{2}$ and $M_{3} . M_{2}$ is the isoform most frequently found in the heart. ACh binding here inhibits adenylate cyclase activity, resulting in a decrease in the excitability of the pacemaker cells, which reduces contractile forces of the atrial cardiac muscle, and decreases conduction velocity of the SA and AV nodes. The net effect of vagal stimulation is therefore a decrease in HR (Bebbington and Brimblecombe 1965). 


\section{$1.5 \quad$ Neural Control of the Heart}

In normal adults, the average HR at rest is approximately 70 beats per minute (bpm). During emotional excitement, muscular activity, or stress, it may accelerate to rates considerably higher than $100 \mathrm{bpm}$ (Berne 2001). The SA node is the pacemaker of the heart, responsible for setting rate and rhythm. The SA node is under the tonic influence of both the sympathetic and parasympathetic nervous systems, such that changes in HR involve a reciprocal action of the two divisions. In healthy resting individuals, parasympathetic tone predominates, which is why the average resting HR is $70 \mathrm{bpm}$ or less. Abolition of parasympathetic influences by administration of atropine increases HR substantially ( $40 \mathrm{bpm}$ ), whereas inhibition of sympathetic effects by administration of propranolol decreases HR only slightly ( $\sim$ bpm) (Katona, McLean et al. 1982). Levy and colleagues further proved that parasympathetic influences prevail at the SA node through constant, simultaneous vagal and sympathetic stimulation in the anesthetized dog (Levy and Zieske 1969). As the frequency of sympathetic stimulation was increased from 0$4 \mathrm{~Hz}$, HR increased by about $80 \mathrm{bpm}$ in the absence of vagal stimulation. However, when the vagus nerve was stimulated at $8 \mathrm{~Hz}$, increasing the sympathetic stimulation frequency had a negligible influence on HR. This vagal domination in the regulation of HR is mediated mainly by functional interactions between the SNS and PNS. Presynaptic interneuronal and postsynaptic intracellular mechanisms have been shown to exist between the terminal postganglionic vagal and sympathetic fibres, which lie in close proximity to one another within the heart. The release of ACh from neighbouring nerve endings causes the effective blockade of the release of norepinephrine from the sympathetic nerve endings thus facilitating the antagonizing activity of the vagus nerve on any concomitant sympathetic activity (Kulbertus 1988).

There is consistent evidence for a reduction in parasympathetic influence as the primary mediator of the increase in HR at the onset of exercise, however the characteristics of sympathetic activity have yet to be clarified. An early study by Robinson et al. (Robinson, Epstein et al. 1966) reported that at low-to-moderate exercise intensity, an increase in HR was mediated primarily by parasympathetic withdrawal, and that sympathetic activity increased initially at $60 \%$ of $\mathrm{VO}_{2 \max }$. In support of this finding, 
Nakamura et al. (Nakamura, Yamamoto et al. 1993) later reported that parasympathetic activity decreased significantly up to $60 \% \mathrm{VO}_{2 \max }$, and sympathetic activity increased significantly after $60 \% \mathrm{VO}_{2 \max }$. Yamamoto and Hughson (Yamamoto, Hughson et al. 1991) further reported parasympathetic activity decreased until exercise intensity reached $60 \%$ of ventilatory threshold. These authors also reported that sympathetic activity remained unchanged up to $100 \%$ of ventilatory threshold and then increased abruptly at $110 \%$. Arai et al. (Arai, Saul et al. 1989) reported a reduction in parasympathetic activity with increasing cycle ergometer intensity, but with an absence of change in sympathetic activity. Therefore, it is important to note that the time course kinetics and relative contribution of the two autonomic divisions differs substantially.

Vagal effects on the heart develop rapidly, often within one heartbeat, and they decay nearly as quickly. Hence, the vagus nerve can exert beat-by-beat control of cardiac function. Conversely, the onset and decay of sympathetic effects are much more gradual; only small changes are affected within the time of one cardiac cycle. For example, during sympathetic cardiac nerve stimulation, the HR and force of contraction increase after a latent period of about 1-3s, approaching an increased steady state level in about 30s (Robertson, Waller et al. 1988). Once stimulation has ended, the return back to baseline takes place much more gradually than at the onset owing to the relatively slow rate of norepinephrine metabolism by the cardiac tissue. Conversely, the changes in HR produced by vagal stimuli appear after a brief latent period (about 50-100ms), reach a steady state response within a few beats, and decay rapidly back to baseline levels (Warner and Cox 1962). As a result, PNS activation is associated with immediate reductions in arterial pressure and HR due to the characteristics of acetylcholinesterase (AChE) and special potassium $\left(\mathrm{K}^{+}\right)$channels in the cardiac cells. $\mathrm{AChE}$ is an enzyme that regulates synaptic ACh concentrations through the hydrolysis of ACh. The molecular mechanisms responsible for the rapid action of the enzyme have yet to be elucidated (Nair, Seravalli et al. 1994). However, it is well known that the release of ACh activates specialized $\mathrm{K}^{+}$channels causing them to open rapidly and effect changes in the pacemaker potential. Thus, very rapid changes in HR are mediated exclusively by the PNS due to the abundance of AChE and consequent rapid clearance of synaptic ACh. 
Taken into context of the current study, moderate intensity IHG exercise of short duration produces a rapid tachycardia in young and healthy individuals at the onset of exercise (Mancia, Iannos et al. 1978, Mark, Victor et al. 1985, Wong, Masse et al. 2007), which pharmacologic evidence indicates is mediated by a decrease in parasympathetic dominance (Hollander and Bouman 1975, Fagraeus and Linnarsson 1976, Mitchell, Reeves et al. 1989). Therefore, the characteristics of the vagal system, in relation to a task known to elicit large and rapid changes in HR, offers a unique opportunity to explore the cortical representation of autonomic cardiovascular control.

\subsubsection{The Baroreflex}

Arterial blood pressure (BP), the product of cardiac output and systemic vascular resistance, is regulated acutely and chronically through various local, humoral, and neural factors. In humans, the major neural pathway by which BP is rapidly and reflexively modulated is called the baroreflex. The baroreflex is a neurocardiovascular reflex that operates in a negative feedback loop to maintain BP homeostasis (Monahan, Dinenno et al. 2001). The purpose of the baroreflex is to regulate mean BP, preventing large transient changes that may arise from sudden stressors (Cowley, Liard et al. 1973). This loop anatomically begins at the level of the carotid and aortic baroreceptors, which are highly specialized stretch-sensitive receptors (a multitude of free nerve endings) located within the wall of the carotid sinus and the aortic arch. These arterial baroreceptors play a key role in the acute control of BP, initiating physiological responses including the modification of HR, vascular resistance, and myocardial contractility (Kaye and Esler 2008). In response to an increase in systemic BP, baroreceptors in the carotid sinus excite fibers in the sinus branch of the glossopharyngeal nerve which project to the nucleus tractus solitarius (NTS), resulting in the inhibition of efferent sympathetic outflow from the rostral ventrolateral medulla (RVLM; primary SNS generator) and excitation of the nucleus ambiguous which initiates vagal outflow. Preganglionic parasympathetic fibers then project to the ganglion cells of the posterior heart where they act to reduce pacemaker activity, thus reducing $\mathrm{HR}$ and therefore BP (Berne 2001). When BP decreases, disinhibition of the RVLM leads to an increase in pre- and postganglionic 
sympathetic activity to the peripheral arterioles, thus increasing the arterial resistance, and effectively increasing HR.

The study of the relationship between changes in BP and HR is used as a measure of the sensitivity of the arterial baroreflex (Monahan, Dinenno et al. 2000). Acute changes in BP reflexively elicit inverse changes in HR via the baroreceptors. This relationship is sigmoidal and the maximum slope of the curve is a key index of baroreflex sensitivity (BRS). Most importantly, the slope of this portion of the curve is principally mediated by PNS activity and accordingly is often termed cardiovagal BRS (Kaye and Esler 2008).

Thus, the baroreflex mechanisms finely tune HR, AV node conduction, myocardial contractility and electrophysiological properties, and peripheral resistance on a beat-bybeat basis, and dampen the effects of perturbations (Eckberg 1992).

\subsubsection{Heart Rate Variability}

As previously mentioned, variation in HR, whether at rest or in response to a stimulus, is mediated by the combined effects of cardiac vagal and sympathetic nerves acting upon the SA node. Heart rate variability (HRV) is a simple and non-invasive measure representing one of the most promising quantitative markers of autonomic balance. HRV describes the oscillations in the interval between consecutive heart beats (RR interval), as well as the oscillations between consecutive instantaneous heart rates. Sinus arrhythmia, or HRV, is enhanced during beta-adrenergic blockade (Coker, Koziell et al. 1984) and is abolished by atropine (Wheeler and Watkins 1973). Although the precise mechanisms responsible for increased variability are not fully understood, the efferent pathway is largely via cardiac vagal fibres.

HRV simultaneously reflects the status of the heart and the reaction of the ANS in compensating for hemodynamic changes and provides a sensible and advanced indicator of health. Thus, HRV can be used to assess the ANS modulation under physiological conditions such as wakefulness and sleep conditions, different body positions, physical training and pathological conditions. High HRV is a signal of efficient adaptation and characterizes competent autonomic mechanisms, while low HRV is frequently an 
indicator of abnormal and insufficient adaptation of the ANS, provoking poor physiological function. Low resting HRV has been related to an increased risk of allcause mortality (Tsuji, Venditti et al. 1994) and to the incidence of ventricular arrhythmias and coronary artery disease (Algra, Tijssen et al. 1993, Tsuji, Larson et al. 1996, Dekker, Schouten et al. 1997, Liao, Cai et al. 1997).

\subsubsection{Statistical Analysis}

The recognition that both the $\mathrm{HR}$ and $\mathrm{BP}$ responses to a variety of stressors exhibit variations on a beat-by-beat scale has led to their evaluation by mathematical analyses, most notably power spectral analyses (Akselrod, Gordon et al. 1985) and the sequence method (Bertinieri, Di Rienzo et al. 1988). These techniques provide an indirect guide to both sympathetic and parasympathetic control of HR and may also be applied to the autonomic control of BP. Using spectral analysis, the HR power spectrum can be divided into low- and high-frequency components. Previous studies using beta-blockade, and atropine demonstrated that the low-frequency oscillations $(0.05-0.1 \mathrm{~Hz})$ in $\mathrm{BP}$ reflect sympathetic modulation of vasomotor tone, and in HR reflect a combination of baroreflex-mediated sympathetic and parasympathetic influences (Smyth, Sleight et al. 1969, Pickering and Davies 1973). High-frequency portions of the power spectrum (0.15$0.5 \mathrm{~Hz})$ are under the primary influence of parasympathetic control; however, the high frequency variability of RR interval is mediated not only by direct modulation of vagal efferent activity resulting from baroreceptor responses to respiratory BP fluctuations (Piepoli, Sleight et al. 1997), but also by mechanical effects of sinus node stretch from respiration-related changes in venous return (Bernardi, Rossi et al. 1989).

The spontaneous cardiovagal baroreflex can also be calculated using the sequence method, which scans BP recordings for sequences of systolic blood pressure (SBP) that are characterized by either increases (or decreases) in SBP of at least $1 \mathrm{mmHg}$ during each of three or more BP waves, and pulse intervals lengthening (or shortening) at least 4 ms/beat. The sequences are then analyzed as linear regressions between the SBP values

and the subsequent pulse intervals. The coefficient of determination $\left(r^{2}\right)$ is taken as the measure of gain or sensitivity of the changes in HR induced by the BP changes, as it is done by the baroreceptor reflex (Bertinieri, Di Rienzo et al. 1988). 


\subsection{The Cortical Autonomic Network}

Although it has been established that the medulla oblongata is the primary cardiovascular control site (Guyenet 2006), studies in rodent models have highlighted the importance of certain forebrain structures involved with the modulation of efferent autonomic outflow (Cechetto and Saper 1987, Dampney 1994, Verberne and Owens 1998). In addition, clinical neuroimaging research has revealed that basal levels of autonomic tone may be disrupted in patients with stroke or epileptic seizures in higher regions of the brain such as the prefrontal and insular cortices, suggesting that cortical regions are involved in cardiovascular regulation (Oppenheimer, Gelb et al. 1992, Cheung and Hachinski 2000, Colivicchi, Bassi et al. 2004). Functional magnetic resonance imaging (fMRI) techniques have enabled the non-invasive assessment of cortical autonomic correlates in healthy, young conscious humans (King, Menon et al. 1999, Critchley, Corfield et al. 2000, Harper, Bandler et al. 2000, Henderson, Macey et al. 2002). A network of forebrain regions, termed the 'cortical autonomic network', has been shown to exert influence over autonomic outflow and cardiovascular control. These regions include the bilateral insular cortex (IC), amygdala, thalamus, medial prefrontal cortex (MPFC), anterior cingulate cortex (ACC), and most recently, the hippocampus (HC).

The IC and ACC are activated during a variety of cognitive maneuvers that elevate autonomic stimulation such as gambling (Critchley, Mathias et al. 2001), Stroop task (Gianaros, Derbyshire et al. 2005), mental arithmetic (Critchley, Corfield et al. 2000) and many more. The involvement of the ACC and IC in influencing sympathetic nerve activity are not only observed during cognitive tasks that induce mental arousal, but are

also reported in situations of physical stress such as baroreceptor unloading (Kimmerly, O'Leary et al. 2005), and isometric exercise (Wong, Masse et al. 2007). Also, neuronal responses have been recorded in the IC when HR and BP changes were elicited by stimulation of the vagus nerve (Barnabi and Cechetto 2001) and baroreflex afferents (Cechetto and Saper 1987). Furthermore, studies in humans have supported a lateralization of insula effects on autonomic cardiovascular control, which is consistent 
with evidence provided from anesthetized rodents. Specifically, HR and BP increase upon stimulation of the right IC, while left IC stimulation produces depressed cardiac and pressor responses (Oppenheimer, Gelb et al. 1992). Experimental studies indicate further that electrical stimulation of the posterior IC, increases HR and BP in anesthetized rats (Ruggiero, Mraovitch et al. 1987), with a large predominance of sympathoexcitatory neurons in the right posterior insula (Oppenheimer and Cechetto 1990, Zhang, Dougherty et al. 1999). Furthermore, during direct stimulation of the rodent brain, the superior IC is associated with tachycardia, whereas inferior portions produce bradycardia (Oppenheimer and Cechetto 1990).

The MPFC has been shown to have strong efferent connections with structures involved in autonomic function including the amygdala, hypothalamus, HC, periaqueductal gray, the NTS and the caudal and rostral ventrolateral medulla (Neafsey 1990, Chiba and Semba 1991, Hurley, Herbert et al. 1991, Verberne and Owens 1998, Vertes 2004). Recent studies conducted in animals and humans have revealed depressor sites within the ventral region of the MPFC that are heightened during periods of relaxation, including sleep and rest (Barnabi and Cechetto 2001, Critchley, Mathias et al. 2001). Furthermore, pharmacological blockade studies (Hollander and Bouman 1975, Mitchell, Reeves et al. 1989, Victor, Pryor et al. 1989) indicate that parasympathetic withdrawal represents the primary determinant of the rapid adjustments in HR that are initiated immediately at the onset of IHG exercise (Mitchell, Reeves et al. 1989, Wong, Masse et al. 2007), and has been shown to be associated with deactivation in the MPFC (Wong, Masse et al. 2007). These observations suggest that the MPFC is involved in mediating behavioural mechanisms that reduce the cardiovascular response to psychological or physical stress, while augmenting vagal efferent control of HR (Critchley, Wiens et al. 2004), thus supporting a direct relation between MPFC activity and cardiovagal control in young, healthy adults.

Emphasis on the role of the $\mathrm{HC}$ in cardiovascular dynamic responses to stress has received relatively little attention despite early studies which noted anatomical visceral sensory connections to the HC (Maclean 1952). More recently, retroviral tracing techniques have established the neuronal linkages and relays that connect brainstem 
autonomic nuclei with the HC (Westerhaus and Loewy 2001, Castle, Comoli et al. 2005). Previously, Ruit and Neafsey (Ruit and Neafsey 1988) illustrated the ability of electrical stimulation of the ventral $\mathrm{HC}$ to depress cardiovascular activation, exposing an inverse relationship between $\mathrm{HC}$ activation and cardiovascular arousal. Of note, the cardiovascular outcomes in this electrical stimulation model required an intact MPFC. These findings confirm early evidence that electrical stimulation of the $\mathrm{HC}$ in anesthetized rats elicits a variety of visceral or autonomic modifications, such as decreases in HR and increases in pulse pressure (Kaada 1951, Kaada and Jasper 1952, Andy and Akert 1955, Liberson and Akert 1955, Anand and DUA 1956). These earlier findings are supported further by recent electroencephalography results from conscious rats that point to entrainment of theta rhythms between the HC and MPFC (Hyman, Hasselmo et al. 2011). Moreover, the necessity of an intact MPFC for electrical stimulation of the HC to elicit cardiovascular changes (Ruit and Neafsey 1988) supports data from conscious humans where cardiac dynamics are inversely related to MPFC activation state in functional neuroimaging studies (Gianaros, Van Der Veen et al. 2004, Wong, Kimmerly et al. 2007, Wong, Masse et al. 2007, Goswami, Frances et al. 2011, Norton, Luchyshyn et al. 2013).

Overall, these observations confirm a network of regions involved in mediating the cardiovascular response to psychological or physical stress, thus supporting a direct relation between cortical activity and cardiovagal control in young, healthy adults. The modulation of this autonomic network through the influence of positive and negative lifestyle factors such as fitness and age, respectively, is not known. 


\subsection{The Effect of Age}

\subsubsection{Effect of Age on the Heart}

The aging process is accompanied by a complex series of changes favoring heightened cardiac sympathetic tone with reduced parasympathetic influence (Meredith, Broughton et al. 1991, Schlaich, Lambert et al. 2004). Within the context of cardiovascular health, chronic elevations in sympathetic outflow, and/or reductions in parasympathetic cardiac control, contribute to the pathogenesis and progression of atherosclerosis, vascular wall thickening, cardiac damage, and disturbed cardiac rhythms (Amiya, Watanabe et al. 2014, Chistiakov, Ashwell et al. 2015, Pellman and Sheikh 2015), which combine to increase the risk of morbidity and mortality (Schmidt, Muller-Werdan et al. 2005).

Age-related changes in maximal exercise $\mathrm{HR}$, and $\mathrm{HR}$ responsiveness, have been reported in the literature (Astrand 1960, Bruce, Blackmon et al. 1963, Lester, Sheffield et al. 1968, Dauchot and Gravenstein 1971, Kino, Lance et al. 1975, Seliger, Macek et al. 1978, Sheffield, Maloof et al. 1978, Yin, Spurgeon et al. 1979, Lalande, Sawicki et al. 2014), and represent a sensitive indicator of autonomic dysfunction. Substantial research has shown that HRV decreases with age (O'Brien, O'Hare et al. 1986, Schwartz, Gibb et al. 1991, Agelink, Malessa et al. 2001, Bonnemeier, Richardt et al. 2003, Russoniello, Zhirnov et al. 2013), which has been observed in both cross-sectional comparisons and longitudinal studies (Sinnreich, Kark et al. 1998). HRV is greater in subjects with the lowest HR and decreased when sympathetic activity increases and vagal activity decreases (Van Hoogenhuyze, Weinstein et al. 1991). It has been shown that the decline in HRV with age is mainly, but not exclusively, attributable to the decline in parasympathetic function (Pfeifer, Weinberg et al. 1983, Shannon, Carley et al. 1987). In addition, respiratory induced changes in HR are reduced in the elderly (Collins, ExtonSmith et al. 1980, Smith, Smith et al. 1981, Smith 1982, Wieling, van Brederode et al. 1982, Taylor, Myers et al. 2001), which may be due to altered vagal activity, since the chronotropic response to atropine is also reduced in older subjects (Dauchot and Gravenstein 1971). However, several other components involved in the genesis of HRV could also be specifically affected by aging including the beta-adrenergic modulation of cardiovascular function (Lakatta 1993, Seals, Taylor et al. 1994, Taylor, Myers et al. 
2001), activity of the renin-angiotensin system (Anderson 1997), and thermoregulation (Kerckhoffs, Blaak et al. 1998).

Advancing age has also been associated with diminished cardiovagal BRS (Robertson, Waller et al. 1988, Ebert, Morgan et al. 1992, Monahan, Dinenno et al. 2001, Monahan 2007, Kaye and Esler 2008), leading to functional changes in BP control and an impaired ability to reflexively engage the cardiovascular system. That is, the degree of HR slowing that occurs with increasing BP is blunted with aging. Traditional thinking has been that increased blood vessel stiffening impairs the function of the afferent baroreceptors in the carotid arteries and aortic arch, through either structural (atherosclerosis) or functional (reduced nitric oxide activity) changes (Robertson, Waller et al. 1988, Hunt, Farquhar et al. 2001). However, Robinson and colleagues found that BRS can be altered acutely by stroke, suggesting that altered central processing can also occur (Robinson, James et al. 1997).

In addition, advancing age often (Ebert, Morgan et al. 1992), but not always (Greaney, Schwartz et al. 2013), increases baseline MSNA. Decreased BRS accounts for some of the decrease in parasympathetic control of HR, as inferred by measures of cardiovagal BRS (Ebert, Morgan et al. 1992, Monahan, Dinenno et al. 2001). However, age exerts little effect on baroreflex control of MSNA whether levels of sympathetic burst activity are elevated (Ebert, Morgan et al. 1992) or not (Greaney, Schwartz et al. 2013).

Combined, these outcomes suggest that advancing age is associated with a chronic state of heightened peripheral sympathetic tone and parasympathetic withdrawal (Meredith, Eisenhofer et al. 1993, Monahan, Dinenno et al. 2001, Thayer and Lane 2007, Lambert, Dawood et al. 2008, Freeling and Li 2015). The mechanisms affecting this autonomic dysregulation are not clear; however, alterations in cerebral cortex structure may contribute to these age-related autonomic changes.

\subsubsection{Effect of Age on the Brain}

The human brain begins to lose tissue early in the third decade of life, with average losses estimated at roughly $15 \%$ of the cerebral cortex and $25 \%$ of the cerebral white matter 
between ages 30 and 90 (Jernigan, Archibald et al. 2001). Major findings include widespread ventricular enlargement (Bradley, Bydder et al. 2002, Fleisher, Sun et al. 2008) and whole brain atrophy (Grubb, Fox et al. 2000), as well as a loss of brain volume in both gray and white matter (Raz, Lindenberger et al. 2005). The prefrontal areas of the brain, which are the last to fully mature in the early twenties, are the first to show signs of decline as early as age forty (Raz, Lindenberger et al. 2005) with degradation of fiber myelination (Northoff, Richter et al. 2000), and a loss of structural connectivity (O'Sullivan, Jones et al. 2001). Significant but more moderate declines are seen in the temporal, parietal and occipital cortices, respectively (Raz and Rodrigue 2006). In addition, the HC, amygdala and cerebellum all show signs of age-related atrophy (Raz and Rodrigue 2006). It is, therefore, noteworthy that those regions associated with autonomic cardiovascular control (e.g. MPFC, IC, HC) exist within regions that display high sensitivity towards age-related cortical atrophy (Raz, Williamson et al. 2000, Anazodo, Shoemaker et al. 2013). In fact, age-related autonomic dysregulation represents one of the many consequences to declines in the integrity of the aged brain (Brody 1955, Cechetto and Shoemaker 2009, Hart, Joyner et al. 2009).

The link between brain structure and function has yet to be fully elucidated. However, advancing age is often associated with changes in brain functional responses (Nyberg, Salami et al. 2010), and declines in cognitive performance (Kramer, Erickson et al. 2006, Prakash, Voss et al. 2011, Voss, Heo et al. 2013) (Ronnlund, Nyberg et al. 2005). Atrophy of the prefrontal cortices in particular, predict a decrease in performance on agesensitive tests of executive function (Raz, Gunning et al. 1997, Gunning-Dixon and Raz 2003) and other cognitive operations dependent on executive control (Head, Rodrigue et al. 2008). This is in line with neuropsychological studies showing that executive functions, which are heavily dependent on frontal neural circuits, are among the cognitive functions most susceptible to advancing age (Connelly, Hasher et al. 1991, Schretlen, Pearlson et al. 2000).

However, although the current literature suggests a consistent age-related decline in cortical structure and function, the rate and extent to which individuals express this decline is highly variable. Erickson et al. (Erickson, Miller et al. 2012) suggest that, at 
least in the context of the $\mathrm{HC}$, exercise as a lifestyle may contribute to this variability. Therefore, despite the overlapping relationship between cognitive and cardiovascular control sites in the brain, the extent to which the inter-individual variation in cortical structure predicts age-related changes in autonomic outflow is not known. If elucidated, details related to cortical structure may form a preliminary basis for explaining agerelated autonomic dysregulation. 


\subsection{The Effect of Exercise}

Over the past several decades, numerous scientific reports have examined the relationship between physical fitness and cardiovascular health. A sedentary lifestyle is one of the five major risk factors for cardiovascular disease, along with high BP, abnormal blood lipids, smoking and obesity (Blair and Brodney 1999). Disturbances in autonomic function are associated with many of the potential mechanisms linked to physical inactivity, including increased BP (Duncan, Farr et al. 1985) and reduced cardiorespiratory fitness (Blair and Brodney 1999). Moreover, the vast majority of patients with autonomic disorders have a blunted or abnormal cardiovascular response to exercise. These patients have a low $\mathrm{VO}_{2 \max }$, indicating reduced physical fitness and exercise capacity (Hilsted, Galbo et al. 1979, Bottini, Tantucci et al. 1995); however, it is likely that the disorder prevents an active lifestyle making direct connections between exercise behavior and autonomic outcomes difficult to interpret and unclear. Physical activity has been shown to improve autonomic balance in older individuals and in patients with cardiovascular disease (Carter and Ray 2015) with altered baseline autonomic function as indicated by heightened SNS and diminished PNS. Thus, understanding how physical (in)activity impacts autonomic control is crucial for sustained cardiovascular health.

\subsubsection{Effect of Acute Exercise on the Heart}

The ANS plays a fundamental role in the cardiovascular response to acute (dynamic) exercise in animals and humans. Successful initiation and persistence of elevated physical activity requires neurally-mediated cardiovascular adjustments that elevate cardiac output, redirect blood flow to the working muscles, and counter the vasodilation in skeletal muscle to prevent hypotension. Input from three neural mechanisms are requisite to precisely match systemic oxygen delivery with metabolic demand: central command, the exercise pressor reflex and the arterial baroreflex (Rowell and O'Leary 1990).

The arterial baroreflex, as previously mentioned, is one of the body's homeostatic mechanisms that works to maintain BP at nearly constant levels. In acute exercise, the operating point of the baroreflex is "reset" to a higher operating systemic pressure 
(Rowell and O'Leary 1990), which allows the baroreflex to operate at the prevailing BP evoked by the exercise (Potts, Shi et al. 1993, Norton, Boushel et al. 1999, Fadel, Ogoh et al. 2001, Ogoh, Fadel et al. 2003, Ogoh, Fisher et al. 2005, Raven, Fadel et al. 2006). There is evidence that both central command and the peripheral feedback from muscle contribute to reset the baroreflex (Iellamo, Legramante et al. 1997, Raven, Fadel et al. 2006).

The term "central command" was coined by Krogh and Lindhard (Krogh and Lindhard 1913) who hypothesized that specific regions of the cortex provided coordinated parallel and concurrent drive to the skeletomotor, respiratory and autonomic neural systems to support muscular activity. At the onset of exercise, central command initiates vagal withdrawal to increase $\mathrm{HR}$ and cardiac output $(\mathrm{CO})$, causing an increase in arterial BP. As work intensity increases and HR approaches 100 beats/min, the demand from active skeletal muscle vasodilation is greater than the increase in $\mathrm{CO}$ and $\mathrm{BP}$, thus initiating a rise in sympathetic nerve activity. A cascade of events involving a further increase in BP and vasoconstriction of inactive vascular beds is initiated through the exercise pressor reflex from sensory afferents of active skeletal muscle (Coote, Hilton et al. 1971, McCloskey and Mitchell 1972, Kaufman, Longhurst et al. 1983, Mitchell, Kaufman et al. 1983).

Therefore, our current understanding of the mechanisms that enable the continuation of high intensity exercise involve central command as the primary regulator of baroreflex resetting, and the feedback mechanism of the exercise pressor reflex as a modulator of this resetting.

\subsubsection{Effect of Exercise Training on the Heart}

The effect of exercise training on cardiac autonomic regulation has been extensively studied in both humans and animals. Several studies provide strong evidence that, under conditions of altered baseline activity, endurance exercise training increases cardiac parasympathetic regulation and decreases sympathetic activation (Gregoire, Tuck et al. 1996, Carter, Banister et al. 2003, Zanesco and Antunes 2007, Billman 2009). These exercise training-induced changes in cardiac autonomic regulation are believed to be 
largely responsible for training-induced decreases in resting HR (Carter, Banister et al. 2003, Zanesco and Antunes 2007). However, the rationale for this exercise-induced resting bradycardia remains controversial. Most, but not all, studies have shown that exercise training consistently increases parasympathetic dominance at rest (Seals and Chase 1989, Scott, Eberhard et al. 2004, Billman and Kukielka 2006, Billman and Kukielka 2007, Billman 2009), whereas intrinsic HR, as revealed by pharmacological blockade, is similar in exercise-trained and sedentary humans or animals (Shi, Stevens et al. 1995, De Angelis, Wichi et al. 2004, Zanesco and Antunes 2007). However, in contrast to longitudinal studies that largely support a predominant autonomic component (Seals and Chase 1989, Shi, Stevens et al. 1995), cross-sectional studies comparing elite athletes with sedentary subjects tend to support an important role for intrinsic HR changes in training bradycardia (Lewis, Nylander et al. 1980, Katona, McLean et al. 1982, Smith, Hudson et al. 1989, Bonaduce, Petretta et al. 1998, Stein, Medeiros et al. 2002).

Endurance training-associated changes in baroreflex control of HR have also been proposed (Stegemann, Busert et al. 1974, Bedford and Tipton 1987, Mack, Shi et al. 1987, Jingu, Takeshita et al. 1988). However, the experimental findings to date have been inconsistent. Chronotropic responsiveness to arterial baroreflex perturbations have been reported to be greater (Monahan, Dinenno et al. 2000), not different (Hudson, Smith et al. 1987, Vroman, Healy et al. 1988, Sheldahl, Ebert et al. 1994, Bowman, Clayton et al. 1997, Davy, Willis et al. 1997), and blunted (Stegemann, Busert et al. 1974, Smith and Raven 1986, Smith, Graitzer et al. 1988) in endurance-trained vs. untrained humans. Importantly, however, regular aerobic exercise has been shown to increase cardiovagal BRS in previously sedentary middle-aged and older adults (Davy, Miniclier et al. 1996, Davy, DeSouza et al. 1998, Monahan, Dinenno et al. 2000). Furthermore, based on the results of a cross-sectional study (Monahan, Dinenno et al. 2000), moderate intensity exercise appears to be an adequate stimulus to attenuate the age-related decline in cardiovagal BRS - no additional benefit was demonstrated in middle-aged and older men who performed more strenuous and prolonged endurance training beyond that of moderate intensity exercise (Monahan, Dinenno et al. 2000). Therefore, there appears to be a dose of training at which the impact of aerobic exercise is transferred to 
improvements in BRS. Three previous intervention studies in middle-aged and older adults found no change in cardiovagal BRS in response to regular aerobic exercise (Sheldahl, Ebert et al. 1994, Bowman, Clayton et al. 1997, Davy, Willis et al. 1997). One potential explanation for this difference is the varied intensity of the exercise stimulus. Specifically, the total volume of the exercise performed in the study by Monahan et al. (5-6 days/week for $\sim 45 \mathrm{~min}$ session for 13.5 weeks at $>70 \%$ maximal HR), was greater than that performed in prior investigations ( $<3$ days/week for $\sim 20-45$ min session for 612 weeks at $70 \%$ maximal HR). Indeed, there is currently no formal training theory that quantitatively and accurately prescribes the pattern, duration and intensity of exercise required to elicit a specific physiological adaptation. In addition, research also indicates that there is individual variability in the response to exercise (Bouchard and Rankinen 2001), and that the training history of an individual will also influence their physiological response.

Considered together, individual differences in physical activity and training-related changes in autonomic outflow suggest that cardiorespiratory fitness is a critical mediator of autonomic adaptation.

\subsubsection{Effect of Exercise on the Brain}

The role of the cortex in the acute adjustment to exercise was first suspected by Krogh and Lindhard (1913) when an anticipatory rise in both HR and respiration was observed prior to the onset of exercise. Pharmacological blockade studies have since supported the idea that the cortex has an important role in adjusting autonomic cardiovascular variables in a manner related to the rapid HR adjustments at the onset of volitional work (McCloskey and Mitchell 1972, Mitchell, Reeves et al. 1989, Victor, Pryor et al. 1989, Mitchell 1990). Subsequently, neuroimaging methods have enabled more detailed studies using volitional exercise with evidence for involvement of the MPFC and IC in modulation of the autonomic nervous system (Williamson, Friedman et al. 1996, Williamson, McColl et al. 1999, Williamson, McColl et al. 2001, Williamson, McColl et al. 2002, Williamson, Fadel et al. 2006, Wong, Masse et al. 2007). 
Considerable change in cortical activation occurs at the onset of moderate intensity exercise where cardiovascular changes appear to be dominated by reductions in parasympathetic contributions. These changes emphasize increased activation within the IC and decreased activation relative to baseline in the MPFC and HC that correlate with HR (Wong, Masse et al. 2007, Norton, Luchyshyn et al. 2013). Importantly, as mentioned previously, these cardiovascular centers are found in regions of the brain most vulnerable to the effects of advancing age (Raz, Gunning et al. 1997, Raz, Lindenberger et al. 2005, Nyberg, Salami et al. 2010, Salami, Eriksson et al. 2012). However, substantial interindividual differences exist in the rate and extent of age-related cortical atrophy leading researchers to examine the potential modifiable risk factors for improved brain health into senescence.

Physical activity has emerged as a potent stimulus for improving neural health and has been shown to have positive global influences in aging, including spared brain volume (Erickson, Prakash et al. 2009, Erickson, Voss et al. 2011, Niemann, Godde et al. 2014), improved task-related functional responses (Colcombe, Kramer et al. 2004, VoelckerRehage, Godde et al. 2010), increased white matter integrity (Johnson, Kim et al. 2012, Voss, Heo et al. 2013, Voss, Weng et al. 2016), and cognitive performance (Josefsson, de Luna et al. 2012). Therefore, the possibility that physical fitness can impact cortical health is of particular importance given that the functional architecture of the cardiovascular control sites, in particular, are negatively altered in advancing age. However, interpretation of the literature remains difficult as studies have previously focused on individuals whose baseline condition, including cognitive impairment or a sedentary lifestyle, represent an effect of such conditions as much as an exercise effect. Further, whether a physically active lifestyle can affect cortical circuitry related to autonomic control and exercise outcomes remains unknown.

A critical aspect of understanding how aerobic activity is protective for brain aging is identifying the fundamental principles by which exercise positively affects brain health. In humans, several studies suggest that physical exercise leads to improvements at both the structural and functional levels in the aging brain (Kramer, Erickson et al. 2006, Hillman, Erickson et al. 2008, Erickson, Prakash et al. 2009) (Voelcker-Rehage, Godde et 
al. 2010, Liu-Ambrose, Nagamatsu et al. 2012). Using voxel-based morphometry, Colcombe et al. (Colcombe, Erickson et al. 2003) reported that a higher cardiorespiratory fitness $\left(\mathrm{VO}_{2 \max }\right)$ was associated with attenuation of cortical decay to both gray and white matter in the frontal, prefrontal, and temporal regions in older adults. In another study, Erickson et al. (Erickson, Prakash et al. 2009) performed a region-of-interest analysis in 165 non-demented older adults and found that higher fitness levels were associated with an increased volume of the bilateral HC. Moreover, Voss et al. (Voss, Prakash et al. 2010) observed that 12 months of aerobic training leads to increased functional connectivity in regional connections that support both the default-mode network and the frontal executive network, suggesting that physical exercise has a restorative effect on large-scale brain circuitry. The evidence supporting a positive influence of fitness on the maintenance and delayed decline of cognitive function is well documented (Hillman, Erickson et al. 2008, Josefsson, de Luna et al. 2012, Bherer, Erickson et al. 2013). Evidence from cross-sectional studies indicates that age-related differences in cognitive performance are observed when older adults are compared to younger adults, and are reduced if the comparisons involve higher-fit individuals rather than sedentary older adults (Spirduso 1975, Clarkson-Smith and Hartley 1989, Hillman, Weiss et al. 2002, Renaud, Bherer et al. 2010). In a meta-analytic review of randomized-control trials of aerobic exercise on neurocognitive functions, Smith et al. found that individuals who were randomly assigned to aerobic exercise training showed modest improvements in attention, processing speed, executive function, and memory (Smith, Blumenthal et al. 2010). Such exercise-induced structural and functional changes have been documented in various brain regions, but the plethora of data has supported alterations in the prefrontal cortex and medial temporal lobes. Thus, the regions of the cortex most vulnerable to the precipitous effects of aging are those same regions where physical activity promotes improvements in cognitive performance. It is noteworthy that these same regions are also responsible for cardiovascular control. Nonetheless, little is known about concurrent autonomic consequences, despite similar, and often times overlapping, regional specificity in the cortex. 


\subsection{Determinants of Cortical Thickness and Brain Function}

The cardiovascular fitness hypothesis suggests that cardiovascular (aerobic) fitness is the key physiological mediator that explains the positive relationship between physical exercise and brain health, with the implication that gains in cardiovascular fitness are necessary for gains in brain health to be observed (Dustman, Ruhling et al. 1984, Barnes, Santos-Modesitt et al. 2013, Brickman, Khan et al. 2014). However, the extent to which exercise can benefit the brain, its impact on autonomic neurologic outcomes, and the mechanisms through which it modulates brain structure and function, remain to be elucidated.

The latest research in both human and non-human animal research demonstrates that exercise targets direct and indirect aspects of neural health leading to broad-based effects on overall brain health. An increase in cell proliferation and cell survival in the dentate gyrus of the $\mathrm{HC}$ is one of the most consistently observed direct effects of exercise training (van Praag, Christie et al. 1999, van Praag, Kempermann et al. 1999, Trejo, Carro et al. 2001, Brown, Cooper-Kuhn et al. 2003, Eadie, Redila et al. 2005). In mice, voluntary wheel running enhanced neurogenesis in the dentate gyrus and was associated with an increased number of new hippocampal cells (van Praag, Kempermann et al. 1999). The functional significance of hippocampal neurogenesis and the survival of new neurons is not clear; however, dementias such as Alzheimer's disease are characterized by a marked reduction in the number of neurons in the $\mathrm{HC}$, which may be alleviated, in part, by increased neurogenesis resulting from aerobic activity (Hillman, Erickson et al. 2008). This proliferation of new cells in the brain is accompanied by an increased demand for nutrients, which is met by the stimulation of new blood vessel growth (angiogenesis) in the cortex (Kleim, Cooper et al. 2002), the cerebellum (Black, Isaacs et al. 1990), and the HC (Lopez-Lopez, LeRoith et al. 2004). A recent imaging study in humans (ages 21-45) showed that 12 weeks of aerobic training increased blood flow in the $\mathrm{HC}$, and this increase was correlated with an improved rate of learning (Pereira, Huddleston et al. 2007). Further, after completion of a 6-month exercise-based cardiac 
rehabilitation program, Anazodo et al. (Anazodo, Shoemaker et al. 2013) observed an increase in cerebral blood flow in the bilateral ACC of CAD patients; an area known to be involved in cognitive processing and regulation of cardiovascular autonomic control.

The predominant theory as to how exercise directly mitigates these effects in the brain is through an increased cortical availability of several classes of growth factors that modulate nearly all of the functional endpoints enhanced by exercise. At present, brainderived neurotrophic factor (BDNF), insulin-like growth factor 1 (IGF-1) and vascular endothelial-derived growth factor (VEGF) are the principal neurotrophins known to mediate the effects of exercise on the brain. These growth factors work together to produce complementary functional effects, modulating both overlapping and unique aspects of exercise-related benefits in brain plasticity, function, and health. Of all the neurotrophins, BDNF appears to be the most susceptible to regulation by exercise and physical activity (Cotman and Berchtold 2002, Knaepen, Goekint et al. 2010). BDNF has consistently been demonstrated to be necessary for long-term memory formation, and for the growth and survival of new neurons (Vaynman, Ying et al. 2004). In humans, BDNF concentrations are increased after an acute exercise bout (Ferris, Williams et al. 2007) in both young adults and patients with multiple sclerosis (Gold, Schulz et al. 2003). Increases in BDNF in response to an exercise stimulus could be clinically relevant, as serum and cortical concentrations of BDNF are reduced in Alzheimer's and Parkinson's disease, as well as depression, anorexia and many others (Adlard, Perreau et al. 2005, Adlard, Perreau et al. 2005). Therefore, aerobic activity may be neuroprotective through the regulation of growth factor secretion.

In addition, exercise-induced increases in microglia and astrocytes (Ehninger and Kempermann 2003), observed in several brain regions, also might help to maintain enhanced brain health and function with exercise. Astrocytes and associated neuroglia are involved in the maintenance and homeostasis of the central nervous system, and act as secretory cells releasing neurotransmitters and growth factors, which affect various aspects of plasticity and information processing in the central nervous system (Malarkey and Parpura 2008, Reichenbach A. 2010, Parpura, Grubisic et al. 2011, Kettenmann, Kirchhoff et al. 2013, Verkhratsky, Rodriguez et al. 2013, Martineau, Parpura et al. 
2014). Morphometric studies have demonstrated that two types of glia, the oligodendrocytes and the microglia, are most affected by physiological aging with reductions of $\sim 11 \%$ in white matter tissues and $\sim 3 \%$ in cortical volume (Haug and Eggers 1991). The impact of aging on astrocyte number remains controversial however, with some observing no change in the aging human brain (Pelvig, Pakkenberg et al. 2008, Fabricius, Jacobsen et al. 2013) and others presenting an age-dependent increase in expression and hypertrophy (Rodriguez, Terzieva et al. 2013, Sampedro-Piquero, De Bartolo et al. 2014). Exposure of aged mice and rats to physical activity or an enriched environment increased astrocyte expression in hippocampal regions and led to astrocytic hypertrophy (Rodriguez, Terzieva et al. 2013, Sampedro-Piquero, De Bartolo et al. 2014). Importantly, these changes in astroglia coincided with cognitive improvement (Sampedro-Piquero, De Bartolo et al. 2014). Therefore, the significance of changes in glia and astrocytes in response to exercise has not been clearly defined and merits further study.

Despite offering some mechanistic insights, a direct effect of the exercise itself may not be the only, or the primary, mechanism through which brain health is improved. As suggested by Spirduso et al. (Spirduso 2005), exercise may also enhance brain health indirectly by improving related conditions such as stress and sleep, diet, and social exposure. Indeed, factors that reduce neurogenesis, such as insomnia, stress and aging, are associated with diminished performance on spatial learning tasks (Lucassen, Meerlo et al. 2010, Lucassen, Oomen et al. 2015, Mueller, Meerlo et al. 2015). Exercise has also been shown to interact with dietary interventions - increasing the positive effects on brain functioning, and decreasing the unhealthy effects of a high-fat diet. In animals, a high-fat diet reduced the hippocampal concentration of BDNF, but this dietary increase was reversed with exercise (Molteni, Wu et al. 2004). In addition, diets rich in sugar, saturated fats, and calories are considered deleterious for neural function, as they act to elevate levels of oxidative stress and reduce synaptic plasticity and cognitive function (Gomez-Pinilla 2011).

Furthermore, exercise reduces peripheral risk factors such as obesity, diabetes, hypertension and cardiovascular disease, which converge to cause brain dysfunction and 
neurodegeneration. Raji et al. (Raji, Ho et al. 2010) used fMRI to assess gray and white matter atrophy in 94 elderly adults (mean age of 77 years). Results showed that body mass index, fasting plasma insulin, and type 2 diabetes were strongly associated with atrophy of the frontal, temporal, and subcortical regions of the brain. In addition, animal studies have revealed a neuroendocrine and/or metabolic role for BDNF in the periphery (Knaepen, Goekint et al. 2010), suggesting a reduction in food intake, an increase in the oxidation of glucose, lower blood glucose levels, and increased insulin sensitivity with higher concentrations of BDNF (Araya, Orellana et al. 2008).

Taken together, these data indicate that the indirect effects of a positive lifestyle associated with improved fitness and increased physical activity may be associated with marked improvements in brain structure and function, and provide a greater understanding of the underlying mechanisms and consequences of high physical activity.

Finally, specific associations between cardiorespiratory fitness and brain health independent of physical activity need to be considered. One possibility is the strong genetic influence (up to 50\%) on cardiorespiratory fitness and the capacity for traininginduced changes in fitness (Bouchard, An et al. 1999, Bouchard, Sarzynski et al. 2011). Similarly, there may be overlapping genetic predictors of the responsiveness of central and peripheral cellular and vascular systems to regular moderate-to-vigorous physical activity and training. For example, increased distribution of blood flow to the muscle is a very important predictor of cardiorespiratory fitness, and this is influenced by the heart's ability to generate more $\mathrm{CO}$ (i.e. by increases in stroke volume) and to recruit and form new capillaries (angiogenesis) in the muscle. Another important training-related adaptation includes changes in the oxidative capacity of mitochondria in active muscle fibers. Angiogenesis and mitochondrial function also increase in the brain in response to exercise training (Marques-Aleixo, Oliveira et al. 2012, Voss, Heo et al. 2013), and so it is possible that individuals that are genetically predisposed to have relatively higher levels of cardiorespiratory fitness or have greater training-induced increases in fitness will experience the most protection against adverse effects of aging on the brain through these vascular and cellular pathways. 
In conclusion, despite its promise, the ways in which physical activity impacts the rate and prevalence of neural decline is still under investigation. Furthermore, several open issues call for further research, such as the dose-response relationship, the level of change or protection provided by physical activity, the biological and/or psychological mechanisms by which these effects occur and whether physical activity can be beneficial despite chronic medical conditions and neurological syndromes such as dementia. Although recent advancements in neuroimaging techniques and genetics have opened new research avenues, more research is required to provide definitive answers to these important questions. 


\subsection{References}

Adlard, P. A., V. M. Perreau and C. W. Cotman (2005). "The exercise-induced expression of BDNF within the hippocampus varies across life-span." Neurobiol Aging 26(4): 511-520.

Adlard, P. A., V. M. Perreau, V. Pop and C. W. Cotman (2005). "Voluntary exercise decreases amyloid load in a transgenic model of Alzheimer's disease." J Neurosci 25(17): 4217-4221.

Agelink, M. W., R. Malessa, B. Baumann, T. Majewski, F. Akila, T. Zeit and D. Ziegler (2001). "Standardized tests of heart rate variability: normal ranges obtained from 309 healthy humans, and effects of age, gender, and heart rate." Clin Auton Res 11(2): 99108.

Akselrod, S., D. Gordon, J. B. Madwed, N. C. Snidman, D. C. Shannon and R. J. Cohen (1985). "Hemodynamic regulation: investigation by spectral analysis." Am J Physiol 249(4 Pt 2): H867-875.

Algra, A., J. G. Tijssen, J. R. Roelandt, J. Pool and J. Lubsen (1993). "Contribution of the 24 hour electrocardiogram to the prediction of sudden coronary death." Br Heart J 70(5): 421-427.

Amiya, E., M. Watanabe and I. Komuro (2014). "The Relationship between Vascular Function and the Autonomic Nervous System." Ann Vasc Dis 7(2): 109-119.

Anand, B. K. and S. DUA (1956). "Circulatory and respiratory changes induced by electrical stimulation of limbic system (visceral brain)." J. Neurophysiol 19(5): 393-400.

Anazodo, U. C., J. K. Shoemaker, N. Suskin and K. S. St Lawrence (2013). "An investigation of changes in regional gray matter volume in cardiovascular disease patients, pre and post cardiovascular rehabilitation." Neuroimage. Clin 3: 388-395.

Anderson, S. (1997). "Ageing and the renin-angiotensin system." Nephrol Dial Transplant 12(6): 1093-1094. 
Andy, O. J. and K. Akert (1955). "Seizure patterns induced by electrical stimulation of hippocampal formation in the cat." J Neuropathol Exp Neurol 14(2): 198-213.

Arai, Y., J. P. Saul, P. Albrecht, L. H. Hartley, L. S. Lilly, R. J. Cohen and W. S. Colucci (1989). "Modulation of cardiac autonomic activity during and immediately after exercise." Am J Physiol 256(1 Pt 2): H132-141.

Araya, A. V., X. Orellana and J. Espinoza (2008). "Evaluation of the effect of caloric restriction on serum BDNF in overweight and obese subjects: preliminary evidences." Endocrine 33(3): 300-304.

Astrand, I. (1960). "Aerobic work capacity in men and women with special reference to age." Acta Physiol Scand Suppl 49(169): 1-92.

Barnabi, F. and D. F. Cechetto (2001). "Neurotransmitters in the thalamus relaying visceral input to the insular cortex in the rat." Am J Physiol Regul Integr Comp Physiol 281(5): R1665-1674.

Barnes, D. E., W. Santos-Modesitt, G. Poelke, A. F. Kramer, C. Castro, L. E. Middleton and K. Yaffe (2013). "The Mental Activity and eXercise (MAX) trial: a randomized controlled trial to enhance cognitive function in older adults." JAMA Intern Med 173(9): 797-804.

Barron, K. D. and S. Chokroverty (1993). Anatomy of the autonomic nervous system: brain and brainstem. Clinical Autonomic Disorders, Evaluation and Management. L. PA, Little, Brown and Co: 3-15.

Bebbington, A. and R. W. Brimblecombe (1965). "Muscarinic receptors in the peripheral and central nervous systems." Adv Drug Res 2: 143-172.

Bedford, T. G. and C. M. Tipton (1987). "Exercise training and the arterial baroreflex." $\underline{\mathbf{J}}$ Appl Physiol (1985) 63(5): 1926-1932. 
Bernardi, L., M. Rossi, F. Soffiantino, G. Marti, L. Ricordi, G. Finardi and P. Fratino (1989). "Cross correlation of heart rate and respiration versus deep breathing. Assessment of new test of cardiac autonomic function in diabetes." Diabetes 38(5): 589-596.

Berne, R. M. L., M.N. (2001). Cardiovascular Physiology. Missouri, Mosby, Inc.

Bertinieri, G., M. Di Rienzo, A. Cavallazzi, A. U. Ferrari, A. Pedotti and G. Mancia (1988). "Evaluation of baroreceptor reflex by blood pressure monitoring in unanesthetized cats." Am J Physiol 254(2 Pt 2): H377-383.

Bherer, L., K. I. Erickson and T. Liu-Ambrose (2013). "A review of the effects of physical activity and exercise on cognitive and brain functions in older adults." J Aging Res 2013: 657508.

Billman, G. E. (2009). "Cardiac autonomic neural remodeling and susceptibility to sudden cardiac death: effect of endurance exercise training." Am J Physiol Heart Circ Physiol 297(4): H1171-1193.

Billman, G. E. and M. Kukielka (2006). "Effects of endurance exercise training on heart rate variability and susceptibility to sudden cardiac death: protection is not due to enhanced cardiac vagal regulation." J Appl Physiol (1985) 100(3): 896-906.

Billman, G. E. and M. Kukielka (2007). "Effect of endurance exercise training on heart rate onset and heart rate recovery responses to submaximal exercise in animals susceptible to ventricular fibrillation." J Appl Physiol (1985) 102(1): 231-240.

Black, J. E., K. R. Isaacs, B. J. Anderson, A. A. Alcantara and W. T. Greenough (1990). "Learning causes synaptogenesis, whereas motor activity causes angiogenesis, in cerebellar cortex of adult rats." Proc Natl Acad Sci U S A 87(14): 5568-5572.

Blair, S. N. and S. Brodney (1999). "Effects of physical inactivity and obesity on morbidity and mortality: current evidence and research issues." $\underline{\text { Med Sci Sports Exerc }}$ 31(11 Suppl): S646-662. 
Bonaduce, D., M. Petretta, V. Cavallaro, C. Apicella, A. Ianniciello, M. Romano, R. Breglio and F. Marciano (1998). "Intensive training and cardiac autonomic control in high level athletes." Med Sci Sports Exerc 30(5): 691-696.

Bonnemeier, H., G. Richardt, J. Potratz, U. K. Wiegand, A. Brandes, N. Kluge and H. A. Katus (2003). "Circadian profile of cardiac autonomic nervous modulation in healthy subjects: differing effects of aging and gender on heart rate variability." J Cardiovasc Electrophysiol 14(8): 791-799.

Bottini, P., C. Tantucci, L. Scionti, M. L. Dottorini, E. Puxeddu, G. Reboldi, G. B. Bolli, G. Casucci, F. Santeusanio, C. A. Sorbini and et al. (1995). "Cardiovascular response to exercise in diabetic patients: influence of autonomic neuropathy of different severity." Diabetologia 38(2): 244-250.

Bouchard, C., P. An, T. Rice, J. S. Skinner, J. H. Wilmore, J. Gagnon, L. Perusse, A. S. Leon and D. C. Rao (1999). "Familial aggregation of VO(2max) response to exercise training: results from the HERITAGE Family Study." J Appl Physiol (1985) 87(3): 10031008.

Bouchard, C. and T. Rankinen (2001). "Individual differences in response to regular physical activity." Med Sci Sports Exerc 33(6 Suppl): S446-451; discussion S452-443.

Bouchard, C., M. A. Sarzynski, T. K. Rice, W. E. Kraus, T. S. Church, Y. J. Sung, D. C. Rao and T. Rankinen (2011). "Genomic predictors of the maximal O(2) uptake response to standardized exercise training programs." J Appl Physiol (1985) 110(5): 1160-1170.

Bowman, A. J., R. H. Clayton, A. Murray, J. W. Reed, M. M. Subhan and G. A. Ford (1997). "Effects of aerobic exercise training and yoga on the baroreflex in healthy elderly persons." Eur J Clin Invest 27(5): 443-449.

Bradley, K. M., G. M. Bydder, M. M. Budge, J. V. Hajnal, S. J. White, B. D. Ripley and A. D. Smith (2002). "Serial brain MRI at 3-6 month intervals as a surrogate marker for Alzheimer's disease." Br J Radiol 75(894): 506-513. 
Brickman, A. M., U. A. Khan, F. A. Provenzano, L. K. Yeung, W. Suzuki, H. Schroeter, M. Wall, R. P. Sloan and S. A. Small (2014). "Enhancing dentate gyrus function with dietary flavanols improves cognition in older adults." Nat Neurosci 17(12): 1798-1803.

Brody, H. (1955). "Organization of the cerebral cortex. III. A study of aging in the human cerebral cortex." J Comp Neurol 102(2): 511-516.

Brown, J., C. M. Cooper-Kuhn, G. Kempermann, H. Van Praag, J. Winkler, F. H. Gage and H. G. Kuhn (2003). "Enriched environment and physical activity stimulate hippocampal but not olfactory bulb neurogenesis." Eur J Neurosci 17(10): 2042-2046.

Bruce, R. A., J. R. Blackmon, J. W. Jones and G. Strait (1963). "Exercising Testing in Adult Normal Subjects and Cardiac Patients." Pediatrics 32: SUPPL 742-756.

Carter, J. B., E. W. Banister and A. P. Blaber (2003). "Effect of endurance exercise on autonomic control of heart rate." Sports Med 33(1): 33-46.

Carter, J. R. and C. A. Ray (2015). "Sympathetic neural adaptations to exercise training in humans." Auton Neurosci 188: 36-43.

Castle, M., E. Comoli and A. D. Loewy (2005). "Autonomic brainstem nuclei are linked to the hippocampus." Neuroscience 134(2): 657-669.

Cechetto, D. F. and S. J. Chen (1990). "Subcortical sites mediating sympathetic responses from insular cortex in rats." Am. J. Physiol 258(1 Pt 2): R245-R255.

Cechetto, D. F. and C. B. Saper (1987). "Evidence for a viscerotopic sensory representation in the cortex and thalamus in the rat." J Comp Neurol 262(1): 27-45.

Cechetto, D. F. and J. K. Shoemaker (2009). "Functional neuroanatomy of autonomic regulation." Neuroimage 47(3): 795-803.

Cheung, R. T. and V. Hachinski (2000). "The insula and cerebrogenic sudden death." Arch. Neurol 57(12): 1685-1688. 
Chiba, T. and R. Semba (1991). "Immuno-electronmicroscopic studies on the gammaaminobutyric acid and glycine receptor in the intermediolateral nucleus of the thoracic spinal cord of rats and guinea pigs." J Auton Nerv Syst 36(3): 173-181.

Chistiakov, D. A., K. W. Ashwell, A. N. Orekhov and Y. V. Bobryshev (2015). "Innervation of the arterial wall and its modification in atherosclerosis." Auton Neurosci 193: $7-11$.

Clarkson-Smith, L. and A. A. Hartley (1989). "Relationships between physical exercise and cognitive abilities in older adults." Psychol Aging 4(2): 183-189.

Coker, R., A. Koziell, C. Oliver and S. E. Smith (1984). "Does the sympathetic nervous system influence sinus arrhythmia in man? Evidence from combined autonomic blockade." J Physiol 356: 459-464.

Colcombe, S. J., K. I. Erickson, N. Raz, A. G. Webb, N. J. Cohen, E. McAuley and A. F. Kramer (2003). "Aerobic fitness reduces brain tissue loss in aging humans." J Gerontol A Biol Sci Med Sci 58(2): 176-180.

Colcombe, S. J., A. F. Kramer, K. I. Erickson, P. Scalf, E. McAuley, N. J. Cohen, A. Webb, G. J. Jerome, D. X. Marquez and S. Elavsky (2004). "Cardiovascular fitness, cortical plasticity, and aging." Proc Natl Acad Sci U S A 101(9): 3316-3321.

Colivicchi, F., A. Bassi, M. Santini and C. Caltagirone (2004). "Cardiac autonomic derangement and arrhythmias in right-sided stroke with insular involvement." $\underline{\text { Stroke }}$ 35(9): 2094-2098.

Collins, K. J., A. N. Exton-Smith, M. H. James and D. J. Oliver (1980). "Functional changes in autonomic nervous responses with ageing." Age Ageing 9(1): 17-24.

Connelly, S. L., L. Hasher and R. T. Zacks (1991). "Age and reading: the impact of distraction." Psychol Aging 6(4): 533-541.

Coote, J. H., S. M. Hilton and J. F. Perez-Gonzalez (1971). "The reflex nature of the pressor response to muscular exercise." J Physiol 215(3): 789-804. 
Cotman, C. W. and N. C. Berchtold (2002). "Exercise: a behavioral intervention to enhance brain health and plasticity." Trends Neurosci 25(6): 295-301.

Cowley, A. W., Jr., J. F. Liard and A. C. Guyton (1973). "Role of baroreceptor reflex in daily control of arterial blood pressure and other variables in dogs." Circ Res 32(5): 564576.

Critchley, H. D., D. R. Corfield, M. P. Chandler, C. J. Mathias and R. J. Dolan (2000). "Cerebral correlates of autonomic cardiovascular arousal: a functional neuroimaging investigation in humans." J. Physiol 523 Pt 1: 259-270.

Critchley, H. D., C. J. Mathias and R. J. Dolan (2001). "Neural activity in the human brain relating to uncertainty and arousal during anticipation." Neuron 29(2): 537-545.

Critchley, H. D., C. J. Mathias, O. Josephs, J. O'Doherty, S. Zanini, B. K. Dewar, L. Cipolotti, T. Shallice and R. J. Dolan (2003). "Human cingulate cortex and autonomic control: converging neuroimaging and clinical evidence." Brain 126(Pt 10): 2139-2152.

Critchley, H. D., Y. Nagai, M. A. Gray and C. J. Mathias (2011). "Dissecting axes of autonomic control in humans: Insights from neuroimaging." Auton Neurosci 161(1-2): 34-42.

Critchley, H. D., P. Rotshtein, Y. Nagai, J. O'Doherty, C. J. Mathias and R. J. Dolan (2005). "Activity in the human brain predicting differential heart rate responses to emotional facial expressions." Neuroimage 24(3): 751-762.

Critchley, H. D., S. Wiens, P. Rotshtein, A. Ohman and R. J. Dolan (2004). "Neural systems supporting interoceptive awareness." Nat Neurosci 7(2): 189-195.

Dampney, R. A. (1994). "Functional organization of central pathways regulating the cardiovascular system." Physiol Rev 74(2): 323-364.

Dauchot, P. and J. S. Gravenstein (1971). "Effects of atropine on the electrocardiogram in different age groups." Clin Pharmacol Ther 12(2): 274-280. 
Davy, K. P., C. A. DeSouza, P. P. Jones and D. R. Seals (1998). "Elevated heart rate variability in physically active young and older adult women." Clin Sci (Lond) 94(6): 579-584.

Davy, K. P., N. L. Miniclier, J. A. Taylor, E. T. Stevenson and D. R. Seals (1996). "Elevated heart rate variability in physically active postmenopausal women: a cardioprotective effect?" Am J Physiol 271(2 Pt 2): H455-460.

Davy, K. P., W. L. Willis and D. R. Seals (1997). "Influence of exercise training on heart rate variability in post-menopausal women with elevated arterial blood pressure." $\underline{\text { Clin }}$ Physiol 17(1): 31-40.

De Angelis, K., R. B. Wichi, W. R. Jesus, E. D. Moreira, M. Morris, E. M. Krieger and M. C. Irigoyen (2004). "Exercise training changes autonomic cardiovascular balance in mice." J Appl Physiol (1985) 96(6): 2174-2178.

Dekker, J. M., E. G. Schouten, P. Klootwijk, J. Pool, C. A. Swenne and D. Kromhout (1997). "Heart rate variability from short electrocardiographic recordings predicts mortality from all causes in middle-aged and elderly men. The Zutphen Study." Am J Epidemiol 145(10): 899-908.

Delius, W., K. E. Hagbarth, A. Hongell and B. G. Wallin (1972). "Manoeuvres affecting sympathetic outflow in human muscle nerves." Acta Physiol Scand 84(1): 82-94.

Duncan, J. J., J. E. Farr, S. J. Upton, R. D. Hagan, M. E. Oglesby and S. N. Blair (1985). "The effects of aerobic exercise on plasma catecholamines and blood pressure in patients with mild essential hypertension." JAMA 254(18): 2609-2613.

Dustman, R. E., R. O. Ruhling, E. M. Russell, D. E. Shearer, H. W. Bonekat, J. W. Shigeoka, J. S. Wood and D. C. Bradford (1984). "Aerobic exercise training and improved neuropsychological function of older individuals." Neurobiol Aging 5(1): 3542. 
Eadie, B. D., V. A. Redila and B. R. Christie (2005). "Voluntary exercise alters the cytoarchitecture of the adult dentate gyrus by increasing cellular proliferation, dendritic complexity, and spine density." J Comp Neurol 486(1): 39-47.

Ebert, T. J., B. J. Morgan, J. A. Barney, T. Denahan and J. J. Smith (1992). "Effects of aging on baroreflex regulation of sympathetic activity in humans." Am J Physiol 263 (3 Pt 2): H798-803.

Eckberg, D. L. S., P. (1992). New Reference. Oxford, Clarendon Press.

Ehninger, D. and G. Kempermann (2003). "Regional effects of wheel running and environmental enrichment on cell genesis and microglia proliferation in the adult murine neocortex." Cereb Cortex 13(8): 845-851.

Erickson, K. I., D. L. Miller and K. A. Roecklein (2012). "The aging hippocampus: interactions between exercise, depression, and BDNF." Neuroscientist 18(1): 82-97.

Erickson, K. I., R. S. Prakash, M. W. Voss, L. Chaddock, L. Hu, K. S. Morris, S. M. White, T. R. Wojcicki, E. McAuley and A. F. Kramer (2009). "Aerobic fitness is associated with hippocampal volume in elderly humans." Hippocampus 19(10): 10301039.

Erickson, K. I., M. W. Voss, R. S. Prakash, C. Basak, A. Szabo, L. Chaddock, J. S. Kim, S. Heo, H. Alves, S. M. White, T. R. Wojcicki, E. Mailey, V. J. Vieira, S. A. Martin, B. D. Pence, J. A. Woods, E. McAuley and A. F. Kramer (2011). "Exercise training increases size of hippocampus and improves memory." Proc Natl Acad Sci U S A 108(7): 3017-3022.

Fabricius, K., J. S. Jacobsen and B. Pakkenberg (2013). "Effect of age on neocortical brain cells in 90+ year old human females--a cell counting study." Neurobiol Aging 34(1): 91-99.

Fadel, P. J., S. Ogoh, D. E. Watenpaugh, W. Wasmund, A. Olivencia-Yurvati, M. L. Smith and P. B. Raven (2001). "Carotid baroreflex regulation of sympathetic nerve 
activity during dynamic exercise in humans." Am J Physiol Heart Circ Physiol 280(3): H1383-1390.

Fagraeus, L. and D. Linnarsson (1976). "Autonomic origin of heart rate fluctuations at the onset of muscular exercise." J. Appl. Physiol 40(5): 679-682.

Ferris, L. T., J. S. Williams and C. L. Shen (2007). "The effect of acute exercise on serum brain-derived neurotrophic factor levels and cognitive function." Med Sci Sports Exerc 39(4): 728-734.

Fleisher, A. S., S. Sun, C. Taylor, C. P. Ward, A. C. Gamst, R. C. Petersen, C. R. Jack, Jr., P. S. Aisen and L. J. Thal (2008). "Volumetric MRI vs clinical predictors of Alzheimer disease in mild cognitive impairment." Neurology 70(3): 191-199.

Ford, G. A. (1999). "Ageing and the baroreflex." Age Ageing 28(4): 337-338.

Freeling, J. L. and Y. Li (2015). "Age-related attenuation of parasympathetic control of the heart in mice." Int J Physiol Pathophysiol Pharmacol 7(3): 126-135.

Gianaros, P. J., S. W. Derbyshire, J. C. May, G. J. Siegle, M. A. Gamalo and J. R. Jennings (2005). "Anterior cingulate activity correlates with blood pressure during stress." Psychophysiology 42(6): 627-635.

Gianaros, P. J., F. M. Van Der Veen and J. R. Jennings (2004). "Regional cerebral blood flow correlates with heart period and high-frequency heart period variability during working-memory tasks: Implications for the cortical and subcortical regulation of cardiac autonomic activity." Psychophysiology 41(4): 521-530.

Gold, S. M., K. H. Schulz, S. Hartmann, M. Mladek, U. E. Lang, R. Hellweg, R. Reer, K. M. Braumann and C. Heesen (2003). "Basal serum levels and reactivity of nerve growth factor and brain-derived neurotrophic factor to standardized acute exercise in multiple sclerosis and controls." J Neuroimmunol 138(1-2): 99-105.

Gomez-Pinilla, F. (2011). "The combined effects of exercise and foods in preventing neurological and cognitive disorders." Prev Med 52 Suppl 1: S75-80. 
Goswami, R., M. F. Frances and J. K. Shoemaker (2011). "Representation of somatosensory inputs within the cortical autonomic network." Neuroimage 54(2): 12111220.

Greaney, J. L., C. E. Schwartz, D. G. Edwards, P. J. Fadel and W. B. Farquhar (2013). "The neural interaction between the arterial baroreflex and muscle metaboreflex is preserved in older men." Exp Physiol 98(10): 1422-1431.

Gregoire, J., S. Tuck, Y. Yamamoto and R. L. Hughson (1996). "Heart rate variability at rest and exercise: influence of age, gender, and physical training." Can J Appl Physiol 21(6): 455-470.

Grubb, N. R., K. A. Fox, K. Smith, J. Best, A. Blane, K. P. Ebmeier, M. F. Glabus and R. E. O'Carroll (2000). "Memory impairment in out-of-hospital cardiac arrest survivors is associated with global reduction in brain volume, not focal hippocampal injury." Stroke 31(7): 1509-1514.

Gunning-Dixon, F. M. and N. Raz (2003). "Neuroanatomical correlates of selected executive functions in middle-aged and older adults: a prospective MRI study." Neuropsychologia 41(14): 1929-1941.

Guyenet, P. G. (2006). "The sympathetic control of blood pressure." Nat Rev Neurosci 7(5): 335-346.

Hachinski, V. C., J. X. Wilson, K. E. Smith and D. F. Cechetto (1992). "Effect of age on autonomic and cardiac responses in a rat stroke model." Arch Neurol 49(7): 690-696.

Hagbarth, K. E. and A. B. Vallbo (1968). "Discharge characteristics of human muscle afferents during muscle stretch and contraction." Exp Neurol 22(4): 674-694.

Harper, R. M., R. Bandler, D. Spriggs and J. R. Alger (2000). "Lateralized and widespread brain activation during transient blood pressure elevation revealed by magnetic resonance imaging." J. Comp Neurol 417(2): 195-204. 
Hart, E. C., M. J. Joyner, B. G. Wallin, C. P. Johnson, T. B. Curry, J. H. Eisenach and N. Charkoudian (2009). "Age-related differences in the sympathetic-hemodynamic balance in men." Hypertension 54(1): 127-133.

Haug, H. and R. Eggers (1991). "Morphometry of the human cortex cerebri and corpus striatum during aging." Neurobiol Aging 12(4): 336-338; discussion 352-335.

Head, D., K. M. Rodrigue, K. M. Kennedy and N. Raz (2008). "Neuroanatomical and cognitive mediators of age-related differences in episodic memory." Neuropsychology 22(4): 491-507.

Henderson, L. A., P. M. Macey, K. E. Macey, R. C. Frysinger, M. A. Woo, R. K. Harper, J. R. Alger, F. L. Yan-Go and R. M. Harper (2002). "Brain responses associated with the Valsalva maneuver revealed by functional magnetic resonance imaging." J Neurophysiol 88(6): 3477-3486.

Hillman, C. H., K. I. Erickson and A. F. Kramer (2008). "Be smart, exercise your heart: exercise effects on brain and cognition." Nat Rev Neurosci 9(1): 58-65.

Hillman, C. H., E. P. Weiss, J. M. Hagberg and B. D. Hatfield (2002). "The relationship of age and cardiovascular fitness to cognitive and motor processes." Psychophysiology 39(3): 303-312.

Hilsted, J., H. Galbo and N. J. Christensen (1979). "Impaired cardiovascular responses to graded exercise in diabetic autonomic neuropathy." Diabetes 28(4): 313-319.

Hollander, A. P. and L. N. Bouman (1975). "Cardiac acceleration in man elicited by a muscle-heart reflex." J. Appl. Physiol 38(2): 272-278.

Hudson, D. L., M. L. Smith and P. B. Raven (1987). "Physical fitness and hemodynamic response of women to lower body negative pressure." Med Sci Sports Exerc 19(4): 375381. 
Hunt, B. E., W. B. Farquhar and J. A. Taylor (2001). "Does reduced vascular stiffening fully explain preserved cardiovagal baroreflex function in older, physically active men?" Circulation 103(20): 2424-2427.

Hurley, K. M., H. Herbert, M. M. Moga and C. B. Saper (1991). "Efferent projections of the infralimbic cortex of the rat." J Comp Neurol 308(2): 249-276.

Hyman, J. M., M. E. Hasselmo and J. K. Seamans (2011). "What is the Functional Relevance of Prefrontal Cortex Entrainment to Hippocampal Theta Rhythms?" Front Neurosci 5: 24.

Iellamo, F., J. M. Legramante, G. Raimondi and G. Peruzzi (1997). "Baroreflex control of sinus node during dynamic exercise in humans: effects of central command and muscle reflexes." Am J Physiol 272(3 Pt 2): H1157-1164.

Izzo, J. L., Jr., R. J. Smith, P. S. Larrabee and M. C. Kallay (1987). "Plasma norepinephrine and age as determinants of systemic hemodynamics in men with established essential hypertension." Hypertension 9(4): 415-419.

Izzo, J. L., Jr. and A. A. Taylor (1999). "The sympathetic nervous system and baroreflexes in hypertension and hypotension." Curr Hypertens Rep 1(3): 254-263.

Jernigan, T. L., S. L. Archibald, C. Fennema-Notestine, A. C. Gamst, J. C. Stout, J. Bonner and J. R. Hesselink (2001). "Effects of age on tissues and regions of the cerebrum and cerebellum." Neurobiol Aging 22(4): 581-594.

Jingu, S., A. Takeshita, T. Imaizumi, M. Nakamura, M. Shindo and H. Tanaka (1988). "Exercise training augments cardiopulmonary baroreflex control of forearm vascular resistance in middle-aged subjects." Jpn Circ J 52(2): 162-168.

Johnson, N. F., C. Kim, J. L. Clasey, A. Bailey and B. T. Gold (2012). "Cardiorespiratory fitness is positively correlated with cerebral white matter integrity in healthy seniors." Neuroimage 59(2): 1514-1523. 
Josefsson, M., X. de Luna, S. Pudas, L. G. Nilsson and L. Nyberg (2012). "Genetic and lifestyle predictors of 15-year longitudinal change in episodic memory." J Am Geriatr Soc 60(12): 2308-2312.

Kaada, B. R. (1951). "Somato-motor, autonomic and electrocorticographic responses to electrical stimulation of rhinencephalic and other structures in primates, cat, and dog; a study of responses from the limbic, subcallosal, orbito-insular, piriform and temporal cortex, hippocampus-fornix and amygdala." Acta Physiol Scand Suppl 24(83): 1-262.

Kaada, B. R. and H. Jasper (1952). "Respiratory responses to stimulation of temporal pole, insula, and hippocampal and limbic gyri in man." AMA Arch Neurol Psychiatry 68(5): 609-619.

Katona, P. G., M. McLean, D. H. Dighton and A. Guz (1982). "Sympathetic and parasympathetic cardiac control in athletes and nonathletes at rest." J Appl Physiol Respir Environ Exerc Physiol 52(6): 1652-1657.

Kaufman, M. P., J. C. Longhurst, K. J. Rybicki, J. H. Wallach and J. H. Mitchell (1983). "Effects of static muscular contraction on impulse activity of groups III and IV afferents in cats." J Appl Physiol Respir Environ Exerc Physiol 55(1 Pt 1): 105-112.

Kaye, D. M. and M. D. Esler (2008). "Autonomic control of the aging heart." Neuromolecular Med 10(3): 179-186.

Kerckhoffs, D. A., E. E. Blaak, M. A. Van Baak and W. H. Saris (1998). "Effect of aging on beta-adrenergically mediated thermogenesis in men." Am J Physiol 274(6 Pt 1): E1075-1079.

Kettenmann, H., F. Kirchhoff and A. Verkhratsky (2013). "Microglia: new roles for the synaptic stripper." Neuron 77(1): 10-18.

Kimmerly, D. S., D. D. O'Leary, R. S. Menon, J. S. Gati and J. K. Shoemaker (2005). "Cortical regions associated with autonomic cardiovascular regulation during lower body negative pressure in humans." J. Physiol 569(Pt 1): 331-345. 
King, A. B., R. S. Menon, V. Hachinski and D. F. Cechetto (1999). "Human forebrain activation by visceral stimuli." J Comp Neurol 413(4): 572-582.

Kino, M., V. Q. Lance, A. Shahamatpour and D. H. Spodick (1975). "Effects of age on responses to isometric exercise. Isometric handgrip in noninvasive screening for cardiovascular disease." Am Heart J 90(5): 575-581.

Kleim, J. A., N. R. Cooper and P. M. VandenBerg (2002). "Exercise induces angiogenesis but does not alter movement representations within rat motor cortex." Brain Res 934(1): 1-6.

Knaepen, K., M. Goekint, E. M. Heyman and R. Meeusen (2010). "Neuroplasticity exercise-induced response of peripheral brain-derived neurotrophic factor: a systematic review of experimental studies in human subjects." Sports Med 40(9): 765-801.

Kramer, A. F., K. I. Erickson and S. J. Colcombe (2006). "Exercise, cognition, and the aging brain." J Appl Physiol (1985) 101(4): 1237-1242.

Krogh, A. and J. Lindhard (1913). "The regulation of respiration and circulation during the initial stages of muscular work." J. Physiol 47(1-2): 112-136.

Kulbertus, H. E. (1988). "Ventricular arrhythmias, syncope and sudden death in aortic stenosis." Eur Heart J 9 Suppl E: 51-52.

Lakatta, E. G. (1993). "Deficient neuroendocrine regulation of the cardiovascular system with advancing age in healthy humans." Circulation 87(2): 631-636.

Lalande, S., C. P. Sawicki, J. R. Baker and J. K. Shoemaker (2014). "Effect of age on the hemodynamic and sympathetic responses at the onset of isometric handgrip exercise." $\underline{\mathbf{J}}$ Appl Physiol (1985) 116(2): 222-227.

Lambert, E., T. Dawood, M. Schlaich, N. Straznicky, M. Esler and G. Lambert (2008). "Single-unit sympathetic discharge pattern in pathological conditions associated with elevated cardiovascular risk." Clin Exp Pharmacol Physiol 35(4): 503-507. 
Lester, M., L. T. Sheffield, P. Trammell and T. J. Reeves (1968). "The effect of age and athletic training on the maximal heart rate during muscular exercise." Am Heart J 76(3): 370-376.

Levy, M. N. and H. Zieske (1969). "Autonomic control of cardiac pacemaker activity and atrioventricular transmission." J Appl Physiol 27(4): 465-470.

Lewis, S. F., E. Nylander, P. Gad and N. H. Areskog (1980). "Non-autonomic component in bradycardia of endurance trained men at rest and during exercise." Acta Physiol Scand 109(3): 297-305.

Liao, D., J. Cai, W. D. Rosamond, R. W. Barnes, R. G. Hutchinson, E. A. Whitsel, P. Rautaharju and G. Heiss (1997). "Cardiac autonomic function and incident coronary heart disease: a population-based case-cohort study. The ARIC Study. Atherosclerosis Risk in Communities Study." Am J Epidemiol 145(8): 696-706.

Liberson, W. T. and K. Akert (1955). "Hippocampal seizure states in guinea pig." Electroencephalogr Clin Neurophysiol 7(2): 211-222.

Liu-Ambrose, T., L. S. Nagamatsu, M. W. Voss, K. M. Khan and T. C. Handy (2012). "Resistance training and functional plasticity of the aging brain: a 12-month randomized controlled trial." Neurobiol Aging 33(8): 1690-1698.

Lopez-Lopez, C., D. LeRoith and I. Torres-Aleman (2004). "Insulin-like growth factor I is required for vessel remodeling in the adult brain." Proc Natl Acad Sci U S A 101(26): 9833-9838.

Lucassen, P. J., P. Meerlo, A. S. Naylor, A. M. van Dam, A. G. Dayer, E. Fuchs, C. A. Oomen and B. Czeh (2010). "Regulation of adult neurogenesis by stress, sleep disruption, exercise and inflammation: Implications for depression and antidepressant action." Eur Neuropsychopharmacol 20(1): 1-17.

Lucassen, P. J., C. A. Oomen, E. F. Naninck, C. P. Fitzsimons, A. M. van Dam, B. Czeh and A. Korosi (2015). "Regulation of Adult Neurogenesis and Plasticity by (Early) 
Stress, Glucocorticoids, and Inflammation." Cold Spring Harb Perspect Biol 7(9): a021303.

Mack, G. W., X. G. Shi, H. Nose, A. Tripathi and E. R. Nadel (1987). "Diminished baroreflex control of forearm vascular resistance in physically fit humans." J Appl Physiol (1985) 63(1): 105-110.

Maclean, P. D. (1952). "Some psychiatric implications of physiological studies on frontotemporal portion of limbic system (visceral brain)." Electroencephalogr Clin Neurophysiol 4(4): 407-418.

Malarkey, E. B. and V. Parpura (2008). "Mechanisms of glutamate release from astrocytes." Neurochem Int 52(1-2): 142-154.

Mancia, G., J. Cleroux, A. Daffonchio, A. U. Ferrari, C. Giannattasio and G. Grassi (1991). "Reflex control of circulation in the elderly." Cardiovasc. Drugs Ther 4 Suppl 6: 1223-1228.

Mancia, G., J. Iannos, G. G. Jamieson, R. H. Lawrence, P. R. Sharman and J. Ludbrook (1978). "Effect of isometric hand-grip exercise on the carotid sinus baroreceptor reflex in man." Clin. Sci. Mol. Med 54(1): 33-37.

Marieb E, M. J., Wilhelm P. (2004). Human Anatomy., Benjamin Cummings.

Mark, A. L., R. G. Victor, C. Nerhed and B. G. Wallin (1985). "Microneurographic studies of the mechanisms of sympathetic nerve responses to static exercise in humans." Circ. Res 57(3): 461-469.

Marques-Aleixo, I., P. J. Oliveira, P. I. Moreira, J. Magalhaes and A. Ascensao (2012). "Physical exercise as a possible strategy for brain protection: evidence from mitochondrial-mediated mechanisms." Prog Neurobiol 99(2): 149-162.

Martineau, M., V. Parpura and J. P. Mothet (2014). "Cell-type specific mechanisms of Dserine uptake and release in the brain." Front Synaptic Neurosci 6: 12. 
McCloskey, D. I. and J. H. Mitchell (1972). "Reflex cardiovascular and respiratory responses originating in exercising muscle." J Physiol 224(1): 173-186.

Meredith, I. T., A. Broughton, G. L. Jennings and M. D. Esler (1991). "Evidence of a selective increase in cardiac sympathetic activity in patients with sustained ventricular arrhythmias." N Engl J Med 325(9): 618-624.

Meredith, I. T., G. Eisenhofer, G. W. Lambert, E. M. Dewar, G. L. Jennings and M. D. Esler (1993). "Cardiac sympathetic nervous activity in congestive heart failure. Evidence for increased neuronal norepinephrine release and preserved neuronal uptake." Circulation 88(1): 136-145.

Mitchell, J. H. (1990). "J.B. Wolffe memorial lecture. Neural control of the circulation during exercise." Med Sci Sports Exerc 22(2): 141-154.

Mitchell, J. H., M. P. Kaufman and G. A. Iwamoto (1983). "The exercise pressor reflex: its cardiovascular effects, afferent mechanisms, and central pathways." Annu Rev Physiol 45: 229-242.

Mitchell, J. H., D. R. Reeves, Jr., H. B. Rogers, N. H. Secher and R. G. Victor (1989). "Autonomic blockade and cardiovascular responses to static exercise in partially curarized man." J. Physiol 413: 433-445.

Molteni, R., A. Wu, S. Vaynman, Z. Ying, R. J. Barnard and F. Gomez-Pinilla (2004). "Exercise reverses the harmful effects of consumption of a high-fat diet on synaptic and behavioral plasticity associated to the action of brain-derived neurotrophic factor." Neuroscience 123(2): 429-440.

Monahan, K. D. (2007). "Effect of aging on baroreflex function in humans." Am J Physiol Regul Integr Comp Physiol 293(1): R3-R12.

Monahan, K. D., F. A. Dinenno, D. R. Seals, C. M. Clevenger, C. A. DeSouza and H. Tanaka (2001). "Age-associated changes in cardiovagal baroreflex sensitivity are related to central arterial compliance." Am. J. Physiol Heart Circ. Physiol 281(1): H284-H289. 
Monahan, K. D., F. A. Dinenno, H. Tanaka, C. M. Clevenger, C. A. DeSouza and D. R. Seals (2000). "Regular aerobic exercise modulates age-associated declines in cardiovagal baroreflex sensitivity in healthy men." J. Physiol 529 Pt 1: 263-271.

Mueller, A. D., P. Meerlo, D. McGinty and R. E. Mistlberger (2015). "Sleep and adult neurogenesis: implications for cognition and mood." Curr Top Behav Neurosci 25: 151181.

Nair, H. K., J. Seravalli, T. Arbuckle and D. M. Quinn (1994). "Molecular recognition in acetylcholinesterase catalysis: free-energy correlations for substrate turnover and inhibition by trifluoro ketone transition-state analogs." Biochemistry 33(28): 8566-8576.

Nakamura, Y., Y. Yamamoto and I. Muraoka (1993). "Autonomic control of heart rate during physical exercise and fractal dimension of heart rate variability." J Appl Physiol (1985) 74(2): 875-881.

Neafsey, E. J. (1990). "Prefrontal cortical control of the autonomic nervous system: anatomical and physiological observations." Prog Brain Res 85: 147-165; discussion 165146.

Nicolini, P., M. M. Ciulla, G. Malfatto, C. Abbate, D. Mari, P. D. Rossi, E. Pettenuzzo, F. Magrini, D. Consonni and F. Lombardi (2014). "Autonomic dysfunction in mild cognitive impairment: evidence from power spectral analysis of heart rate variability in a cross-sectional case-control study." PLoS One 9(5): e96656.

Niemann, C., B. Godde and C. Voelcker-Rehage (2014). "Not only cardiovascular, but also coordinative exercise increases hippocampal volume in older adults." Front Aging Neurosci 6: 170.

Ninomiya, I., S. C. Malpas, K. Matsukawa, T. Shindo and T. Akiyama (1993). "The amplitude of synchronized cardiac sympathetic nerve activity reflects the number of activated pre- and postganglionic fibers in anesthetized cats." J Auton Nerv Syst $\mathbf{4 5}(2)$ : 139-147. 
Norris, J. W., G. M. Froggatt and V. C. Hachinski (1978). "Cardiac arrhythmias in acute stroke." Stroke 9(4): 392-396.

Northoff, G., A. Richter, M. Gessner, F. Schlagenhauf, J. Fell, F. Baumgart, T. Kaulisch, R. Kotter, K. E. Stephan, A. Leschinger, T. Hagner, B. Bargel, T. Witzel, H. Hinrichs, B. Bogerts, H. Scheich and H. J. Heinze (2000). "Functional dissociation between medial and lateral prefrontal cortical spatiotemporal activation in negative and positive emotions: a combined fMRI/MEG study." Cereb Cortex 10(1): 93-107.

Norton, K. H., R. Boushel, S. Strange, B. Saltin and P. B. Raven (1999). "Resetting of the carotid arterial baroreflex during dynamic exercise in humans." J Appl Physiol (1985) 87(1): 332-338.

Norton, K. N., T. A. Luchyshyn and S. J. Kevin (2013). "Evidence for a medial prefrontal cortex-hippocampal axis associated with heart rate control in conscious humans." Brain Res 1538: 104-115.

Nyberg, L., M. Lovden, K. Riklund, U. Lindenberger and L. Backman (2012). "Memory aging and brain maintenance." Trends Cogn Sci 16(5): 292-305.

Nyberg, L., A. Salami, M. Andersson, J. Eriksson, G. Kalpouzos, K. Kauppi, J. Lind, S. Pudas, J. Persson and L. G. Nilsson (2010). "Longitudinal evidence for diminished frontal cortex function in aging." Proc Natl Acad Sci U S A 107(52): 22682-22686.

O'Brien, I. A., P. O'Hare and R. J. Corrall (1986). "Heart rate variability in healthy subjects: effect of age and the derivation of normal ranges for tests of autonomic function." Br Heart J 55(4): 348-354.

O'Sullivan, M., D. K. Jones, P. E. Summers, R. G. Morris, S. C. Williams and H. S. Markus (2001). "Evidence for cortical "disconnection" as a mechanism of age-related cognitive decline." Neurology 57(4): 632-638.

Ogoh, S., P. J. Fadel, P. Nissen, O. Jans, C. Selmer, N. H. Secher and P. B. Raven (2003). "Baroreflex-mediated changes in cardiac output and vascular conductance in response to 


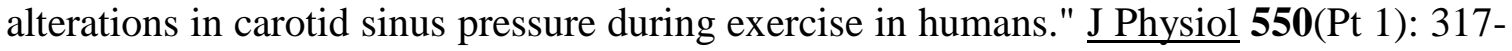
324.

Ogoh, S., J. P. Fisher, E. A. Dawson, M. J. White, N. H. Secher and P. B. Raven (2005). "Autonomic nervous system influence on arterial baroreflex control of heart rate during exercise in humans." J Physiol 566(Pt 2): 599-611.

Olshansky, B., H. N. Sabbah, P. J. Hauptman and W. S. Colucci (2008). "Parasympathetic nervous system and heart failure: pathophysiology and potential implications for therapy." Circulation 118(8): 863-871.

Oppenheimer, S. M. and D. F. Cechetto (1990). "Cardiac chronotropic organization of the rat insular cortex." Brain Res 533(1): 66-72.

Oppenheimer, S. M., A. Gelb, J. P. Girvin and V. C. Hachinski (1992). "Cardiovascular effects of human insular cortex stimulation." Neurology 42(9): 1727-1732.

Parpura, V., V. Grubisic and A. Verkhratsky (2011). "Ca(2+) sources for the exocytotic release of glutamate from astrocytes." Biochim Biophys Acta 1813(5): 984-991.

Pellman, J. and F. Sheikh (2015). "Atrial fibrillation: mechanisms, therapeutics, and future directions." Compr Physiol 5(2): 649-665.

Pelvig, D. P., H. Pakkenberg, A. K. Stark and B. Pakkenberg (2008). "Neocortical glial cell numbers in human brains." Neurobiol Aging 29(11): 1754-1762.

Pereira, A. C., D. E. Huddleston, A. M. Brickman, A. A. Sosunov, R. Hen, G. M. McKhann, R. Sloan, F. H. Gage, T. R. Brown and S. A. Small (2007). "An in vivo correlate of exercise-induced neurogenesis in the adult dentate gyrus." Proc Natl Acad Sci U S A 104(13): 5638-5643.

Pfeifer, M. A., C. R. Weinberg, D. Cook, J. D. Best, A. Reenan and J. B. Halter (1983). "Differential changes of autonomic nervous system function with age in man." Am J Med 75(2): 249-258. 
Pickering, T. G. and J. Davies (1973). "Estimation of the conduction time of the baroreceptor-cardiac reflex in man." Cardiovasc Res 7(2): 213-219.

Piepoli, M., P. Sleight, S. Leuzzi, F. Valle, G. Spadacini, C. Passino, J. Johnston and L. Bernardi (1997). "Origin of respiratory sinus arrhythmia in conscious humans. An important role for arterial carotid baroreceptors." Circulation 95(7): 1813-1821.

Potts, J. T., X. R. Shi and P. B. Raven (1993). "Carotid baroreflex responsiveness during dynamic exercise in humans." Am J Physiol 265(6 Pt 2): H1928-1938.

Prakash, R. S., M. W. Voss, K. I. Erickson, J. M. Lewis, L. Chaddock, E. Malkowski, H. Alves, J. Kim, A. Szabo, S. M. White, T. R. Wojcicki, E. L. Klamm, E. McAuley and A. F. Kramer (2011). "Cardiorespiratory fitness and attentional control in the aging brain." Front Hum Neurosci 4: 229.

Pudas, S., J. Persson, M. Josefsson, X. de Luna, L. G. Nilsson and L. Nyberg (2013). "Brain characteristics of individuals resisting age-related cognitive decline over two decades." J Neurosci 33(20): 8668-8677.

Raji, C. A., A. J. Ho, N. N. Parikshak, J. T. Becker, O. L. Lopez, L. H. Kuller, X. Hua, A. D. Leow, A. W. Toga and P. M. Thompson (2010). "Brain structure and obesity." Hum Brain Mapp 31(3): 353-364.

Raven, P. B., P. J. Fadel and S. Ogoh (2006). "Arterial baroreflex resetting during exercise: a current perspective." Exp Physiol 91(1): 37-49.

Raz, N., F. M. Gunning, D. Head, J. H. Dupuis, J. McQuain, S. D. Briggs, W. J. Loken, A. E. Thornton and J. D. Acker (1997). "Selective aging of the human cerebral cortex observed in vivo: differential vulnerability of the prefrontal gray matter." Cereb. Cortex 7(3): 268-282.

Raz, N., U. Lindenberger, K. M. Rodrigue, K. M. Kennedy, D. Head, A. Williamson, C. Dahle, D. Gerstorf and J. D. Acker (2005). "Regional brain changes in aging healthy adults: general trends, individual differences and modifiers." Cereb. Cortex 15(11): 16761689. 
Raz, N. and K. M. Rodrigue (2006). "Differential aging of the brain: patterns, cognitive correlates and modifiers." Neurosci Biobehav Rev 30(6): 730-748.

Raz, N., A. Williamson, F. Gunning-Dixon, D. Head and J. D. Acker (2000). "Neuroanatomical and cognitive correlates of adult age differences in acquisition of a perceptual-motor skill." Microsc Res Tech 51(1): 85-93.

Reichenbach A., B. A. (2010). Muller cells in the healthy and

diseased retina. . Heidelberg, Springer.

Renaud, M., L. Bherer and F. Maquestiaux (2010). "A high level of physical fitness is associated with more efficient response preparation in older adults." J Gerontol B Psychol Sci Soc Sci 65B(3): 317-322.

Robertson, D. R., D. G. Waller, A. G. Renwick and C. F. George (1988). "Age-related changes in the pharmacokinetics and pharmacodynamics of nifedipine." $\underline{\mathrm{Br} \mathrm{J} \text { Clin }}$ Pharmacol 25(3): 297-305.

Robinson, B. F., S. E. Epstein, G. D. Beiser and E. Braunwald (1966). "Control of heart rate by the autonomic nervous system. Studies in man on the interrelation between baroreceptor mechanisms and exercise." Circ Res 19(2): 400-411.

Robinson, T. G., M. James, J. Youde, R. Panerai and J. Potter (1997). "Cardiac baroreceptor sensitivity is impaired after acute stroke." Stroke 28(9): 1671-1676.

Rodriguez, J. J., S. Terzieva, M. Olabarria, R. G. Lanza and A. Verkhratsky (2013). "Enriched environment and physical activity reverse astrogliodegeneration in the hippocampus of AD transgenic mice." Cell Death Dis 4: e678.

Ronnlund, M., L. Nyberg, L. Backman and L. G. Nilsson (2005). "Stability, growth, and decline in adult life span development of declarative memory: cross-sectional and longitudinal data from a population-based study." Psychol Aging 20(1): 3-18. 
Rowell, L. B. and D. S. O'Leary (1990). "Reflex control of the circulation during exercise: chemoreflexes and mechanoreflexes." J Appl Physiol (1985) 69(2): 407-418.

Ruggiero, D. A., S. Mraovitch, A. R. Granata, M. Anwar and D. J. Reis (1987). "A role of insular cortex in cardiovascular function." J. Comp Neurol 257(2): 189-207.

Ruit, K. G. and E. J. Neafsey (1988). "Cardiovascular and respiratory responses to electrical and chemical stimulation of the hippocampus in anesthetized and awake rats." Brain Res 457(2): 310-321.

Russoniello, C. V., Y. N. Zhirnov, V. I. Pougatchev and E. N. Gribkov (2013). "Heart rate variability and biological age: implications for health and gaming." Cyberpsychol Behav Soc Netw 16(4): 302-308.

Salami, A., J. Eriksson and L. Nyberg (2012). "Opposing effects of aging on large-scale

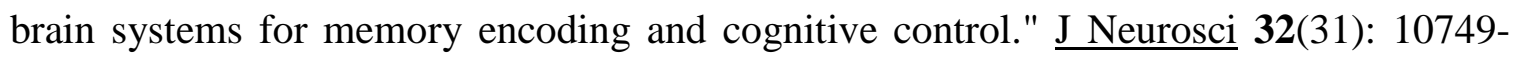
10757.

Salmanpour, A., L. J. Brown, C. D. Steinback, C. W. Usselman, R. Goswami and J. K. Shoemaker (2011). "Relationship between size and latency of action potentials in human muscle sympathetic nerve activity." J Neurophysiol 105(6): 2830-2842.

Sampedro-Piquero, P., P. De Bartolo, L. Petrosini, C. Zancada-Menendez, J. L. Arias and A. Begega (2014). "Astrocytic plasticity as a possible mediator of the cognitive improvements after environmental enrichment in aged rats." Neurobiol Learn Mem 114: $16-25$.

Schlaich, M. P., E. Lambert, D. M. Kaye, Z. Krozowski, D. J. Campbell, G. Lambert, J. Hastings, A. Aggarwal and M. D. Esler (2004). "Sympathetic augmentation in hypertension: role of nerve firing, norepinephrine reuptake, and Angiotensin neuromodulation." Hypertension 43(2): 169-175.

Schmidt, H., U. Muller-Werdan, T. Hoffmann, D. P. Francis, M. F. Piepoli, M. Rauchhaus, R. Prondzinsky, H. Loppnow, M. Buerke, D. Hoyer and K. Werdan (2005). 
"Autonomic dysfunction predicts mortality in patients with multiple organ dysfunction syndrome of different age groups." Crit Care Med 33(9): 1994-2002.

Schretlen, D., G. D. Pearlson, J. C. Anthony, E. H. Aylward, A. M. Augustine, A. Davis and P. Barta (2000). "Elucidating the contributions of processing speed, executive ability, and frontal lobe volume to normal age-related differences in fluid intelligence." $\underline{\mathrm{J} \text { Int }}$ Neuropsychol Soc 6(1): 52-61.

Schwartz, J. B., W. J. Gibb and T. Tran (1991). "Aging effects on heart rate variation." $\underline{J}$ Gerontol 46(3): M99-106.

Scott, A. S., A. Eberhard, D. Ofir, G. Benchetrit, T. P. Dinh, P. Calabrese, V. Lesiuk and H. Perrault (2004). "Enhanced cardiac vagal efferent activity does not explain traininginduced bradycardia." Auton Neurosci 112(1-2): 60-68.

Seals, D. R. and P. B. Chase (1989). "Influence of physical training on heart rate variability and baroreflex circulatory control." J Appl Physiol (1985) 66(4): 1886-1895.

Seals, D. R., J. A. Taylor, A. V. Ng and M. D. Esler (1994). "Exercise and aging: autonomic control of the circulation." Med. Sci. Sports Exerc 26(5): 568-576.

Seliger, V., M. Macek, O. Skranc, J. Horak, J. Piric, P. Handzo, J. Rous and Z. Jirka (1978). "Work capacity of the Czechoslovakian population." Eur J Appl Physiol Occup Physiol 39(3): 155-164.

Shannon, D. C., D. W. Carley and H. Benson (1987). "Aging of modulation of heart rate." Am J Physiol 253(4 Pt 2): H874-877.

Sheffield, L. T., J. A. Maloof, J. A. Sawyer and D. Roitman (1978). "Maximal heart rate and treadmill performance of healthy women in relation to age." Circulation 57(1): 7984.

Sheldahl, L. M., T. J. Ebert, B. Cox and F. E. Tristani (1994). "Effect of aerobic training on baroreflex regulation of cardiac and sympathetic function." J Appl Physiol (1985) 76(1): 158-165. 
Shi, X., G. H. Stevens, B. H. Foresman, S. A. Stern and P. B. Raven (1995). "Autonomic nervous system control of the heart: endurance exercise training." Med Sci Sports Exerc 27(10): 1406-1413.

Shields, R. W., Jr. (1993). "Functional anatomy of the autonomic nervous system." J Clin Neurophysiol 10(1): 2-13.

Shoemaker, J. K. and R. Goswami (2015). "Forebrain neurocircuitry associated with human reflex cardiovascular control." Front Physiol 6: 240.

Sinnreich, R., J. D. Kark, Y. Friedlander, D. Sapoznikov and M. H. Luria (1998). "Five minute recordings of heart rate variability for population studies: repeatability and agesex characteristics." Heart 80(2): 156-162.

Smith, M. L., H. M. Graitzer, D. L. Hudson and P. B. Raven (1988). "Baroreflex function in endurance- and static exercise-trained men." J Appl Physiol (1985) 64(2): 585-591.

Smith, M. L., D. L. Hudson, H. M. Graitzer and P. B. Raven (1989). "Exercise training bradycardia: the role of autonomic balance." Med Sci Sports Exerc 21(1): 40-44.

Smith, M. L. and P. B. Raven (1986). "Cardiovascular responses to lower body negative pressure in endurance and static exercise-trained men." Med Sci Sports Exerc 18(5): 545550.

Smith, P. J., J. A. Blumenthal, B. M. Hoffman, H. Cooper, T. A. Strauman, K. WelshBohmer, J. N. Browndyke and A. Sherwood (2010). "Aerobic exercise and neurocognitive performance: a meta-analytic review of randomized controlled trials." Psychosom Med 72(3): 239-252.

Smith, S. A. (1982). "Reduced sinus arrhythmia in diabetic autonomic neuropathy: diagnostic value of an age-related normal range." Br Med J (Clin Res Ed) 285(6355): 1599-1601.

Smith, S. E., S. A. Smith and P. M. Brown (1981). "Cardiac autonomic dysfunction in patients with diabetic retinopathy." Diabetologia 21(6): 525-528. 
Smyth, H. S., P. Sleight and G. W. Pickering (1969). "Reflex regulation of arterial pressure during sleep in man. A quantitative method of assessing baroreflex sensitivity." Circ Res 24(1): 109-121.

Soros, P. and V. Hachinski (2012). "Cardiovascular and neurological causes of sudden death after ischaemic stroke." Lancet Neurol 11(2): 179-188.

Soufer, R., J. D. Bremner, J. A. Arrighi, I. Cohen, B. L. Zaret, M. M. Burg and P. Goldman-Rakic (1998). "Cerebral cortical hyperactivation in response to mental stress in patients with coronary artery disease." Proc Natl Acad Sci U S A 95(11): 6454-6459.

Spirduso, W. W. (1975). "Reaction and movement time as a function of age and physical activity level." J Gerontol 30(4): 435-440.

Spirduso, W. W., Francis K., MacRae P. (2005). Physical Dimensions of Aging. Champaign, IL, Human Kinetics.

Stegemann, J., A. Busert and D. Brock (1974). "Influence of fitness on the blood pressure control system in man." Aerosp Med 45(1): 45-48.

Stein, R., C. M. Medeiros, G. A. Rosito, L. I. Zimerman and J. P. Ribeiro (2002). "Intrinsic sinus and atrioventricular node electrophysiologic adaptations in endurance athletes." J Am Coll Cardiol 39(6): 1033-1038.

Taylor, J. A., C. W. Myers, J. R. Halliwill, H. Seidel and D. L. Eckberg (2001). "Sympathetic restraint of respiratory sinus arrhythmia: implications for vagal-cardiac tone assessment in humans." Am J Physiol Heart Circ Physiol 280(6): H2804-2814.

Thayer, J. F. and R. D. Lane (2007). "The role of vagal function in the risk for cardiovascular disease and mortality." Biol Psychol 74(2): 224-242.

Thayer, J. F., J. J. Sollers, III, D. M. Labiner, M. Weinand, A. M. Herring, R. D. Lane and G. L. Ahern (2009). "Age-related differences in prefrontal control of heart rate in humans: a pharmacological blockade study." Int. J. Psychophysiol 72(1): 81-88. 
Tompkins, R. P., C. W. Melling, T. D. Wilson, B. D. Bates and J. K. Shoemaker (2013). "Arrangement of sympathetic fibers within the human common peroneal nerve: implications for microneurography." J Appl Physiol (1985) 115(10): 1553-1561.

Trejo, J. L., E. Carro and I. Torres-Aleman (2001). "Circulating insulin-like growth factor I mediates exercise-induced increases in the number of new neurons in the adult hippocampus." J Neurosci 21(5): 1628-1634.

Tsuji, H., M. G. Larson, F. J. Venditti, Jr., E. S. Manders, J. C. Evans, C. L. Feldman and D. Levy (1996). "Impact of reduced heart rate variability on risk for cardiac events. The Framingham Heart Study." Circulation 94(11): 2850-2855.

Tsuji, H., F. J. Venditti, Jr., E. S. Manders, J. C. Evans, M. G. Larson, C. L. Feldman and D. Levy (1994). "Reduced heart rate variability and mortality risk in an elderly cohort. The Framingham Heart Study." Circulation 90(2): 878-883.

Van Hoogenhuyze, D., N. Weinstein, G. J. Martin, J. S. Weiss, J. W. Schaad, X. N. Sahyouni, D. Fintel, W. J. Remme and D. H. Singer (1991). "Reproducibility and relation to mean heart rate of heart rate variability in normal subjects and in patients with congestive heart failure secondary to coronary artery disease." Am J Cardiol 68(17): 1668-1676.

van Praag, H., B. R. Christie, T. J. Sejnowski and F. H. Gage (1999). "Running enhances neurogenesis, learning, and long-term potentiation in mice." Proc Natl Acad Sci U S A 96(23): 13427-13431.

van Praag, H., G. Kempermann and F. H. Gage (1999). "Running increases cell proliferation and neurogenesis in the adult mouse dentate gyrus." Nat Neurosci 2(3): 266270.

Vaynman, S., Z. Ying and F. Gomez-Pinilla (2004). "Hippocampal BDNF mediates the efficacy of exercise on synaptic plasticity and cognition." Eur J Neurosci 20(10): 25802590. 
Verberne, A. J. (1996). "Medullary sympathoexcitatory neurons are inhibited by activation of the medial prefrontal cortex in the rat." Am. J. Physiol 270(4 Pt 2): R713R719.

Verberne, A. J. and N. C. Owens (1998). "Cortical Modulation of the Cardiovascular System." Progress in Neurobiology 54(2): 149-168.

Verkhratsky, A., J. J. Rodriguez and V. Parpura (2013). "Astroglia in neurological diseases." Future Neurol 8(2): 149-158.

Vertes, R. P. (2004). "Differential projections of the infralimbic and prelimbic cortex in the rat." Synapse 51(1): 32-58.

Victor, R. G., S. L. Pryor, N. H. Secher and J. H. Mitchell (1989). "Effects of partial neuromuscular blockade on sympathetic nerve responses to static exercise in humans." Circ. Res 65(2): 468-476.

Voelcker-Rehage, C., B. Godde and U. M. Staudinger (2010). "Physical and motor fitness are both related to cognition in old age." Eur J Neurosci 31(1): 167-176.

Voss, M. W., S. Heo, R. S. Prakash, K. I. Erickson, H. Alves, L. Chaddock, A. N. Szabo, E. L. Mailey, T. R. Wojcicki, S. M. White, N. Gothe, E. McAuley, B. P. Sutton and A. F. Kramer (2013). "The influence of aerobic fitness on cerebral white matter integrity and cognitive function in older adults: results of a one-year exercise intervention." Hum. Brain Mapp 34(11): 2972-2985.

Voss, M. W., R. S. Prakash, K. I. Erickson, C. Basak, L. Chaddock, J. S. Kim, H. Alves, S. Heo, A. N. Szabo, S. M. White, T. R. Wojcicki, E. L. Mailey, N. Gothe, E. A. Olson, E. McAuley and A. F. Kramer (2010). "Plasticity of brain networks in a randomized intervention trial of exercise training in older adults." Front Aging Neurosci 2.

Voss, M. W., T. B. Weng, A. Z. Burzynska, C. N. Wong, G. E. Cooke, R. Clark, J. Fanning, E. Awick, N. P. Gothe, E. A. Olson, E. McAuley and A. F. Kramer (2016). "Fitness, but not physical activity, is related to functional integrity of brain networks associated with aging." Neuroimage 131: 113-125. 
Vroman, N. B., J. A. Healy and R. Kertzer (1988). "Cardiovascular response to lower body negative pressure (LBNP) following endurance training." Aviat Space Environ Med 59(4): 330-334.

Warner, H. R. and A. Cox (1962). "A mathematical model of heart rate control by sympathetic and vagus efferent information." J Appl Physiol 17: 349-355.

Westerhaus, M. J. and A. D. Loewy (2001). "Central representation of the sympathetic nervous system in the cerebral cortex." Brain Res 903(1-2): 117-127.

Wheeler, T. and P. J. Watkins (1973). "Cardiac denervation in diabetes." $\underline{\mathrm{Br} \text { Med J }}$ 4(5892): 584-586.

Wieling, W., J. F. van Brederode, L. G. de Rijk, C. Borst and A. J. Dunning (1982). "Reflex control of heart rate in normal subjects in relation to age: a data base for cardiac vagal neuropathy." Diabetologia 22(3): 163-166.

Williamson, J. W., P. J. Fadel and J. H. Mitchell (2006). "New insights into central cardiovascular control during exercise in humans: a central command update." Exp Physiol 91(1): 51-58.

Williamson, J. W., D. B. Friedman, J. H. Mitchell, N. H. Secher and L. Friberg (1996). "Mechanisms regulating regional cerebral activation during dynamic handgrip in humans." J Appl Physiol (1985) 81(5): 1884-1890.

Williamson, J. W., R. McColl, D. Mathews, M. Ginsburg and J. H. Mitchell (1999). "Activation of the insular cortex is affected by the intensity of exercise." J Appl Physiol (1985) 87(3): 1213-1219.

Williamson, J. W., R. McColl, D. Mathews, J. H. Mitchell, P. B. Raven and W. P. Morgan (2001). "Hypnotic manipulation of effort sense during dynamic exercise: cardiovascular responses and brain activation." J Appl Physiol (1985) 90(4): 1392-1399. 
Williamson, J. W., R. McColl, D. Mathews, J. H. Mitchell, P. B. Raven and W. P. Morgan (2002). "Brain activation by central command during actual and imagined handgrip under hypnosis." J Appl Physiol (1985) 92(3): 1317-1324.

Wong, S. W., D. S. Kimmerly, N. Masse, R. S. Menon, D. F. Cechetto and J. K. Shoemaker (2007). "Sex differences in forebrain and cardiovagal responses at the onset of isometric handgrip exercise: a retrospective fMRI study." J. Appl. Physiol 103(4): 1402-1411.

Wong, S. W., N. Masse, D. S. Kimmerly, R. S. Menon and J. K. Shoemaker (2007). "Ventral medial prefrontal cortex and cardiovagal control in conscious humans." Neuroimage 35(2): 698-708.

Woo, M. A., P. M. Macey, G. C. Fonarow, M. A. Hamilton and R. M. Harper (2003). "Regional brain gray matter loss in heart failure." J Appl Physiol (1985) 95(2): 677-684.

Woo, M. A., S. K. Yadav, P. M. Macey, G. C. Fonarow, R. M. Harper and R. Kumar (2014). "Brain metabolites in autonomic regulatory insular sites in heart failure." J Neurol Sci 346(1-2): 271-275.

Yamamoto, Y., R. L. Hughson and J. C. Peterson (1991). "Autonomic control of heart rate during exercise studied by heart rate variability spectral analysis." J Appl Physiol (1985) 71(3): 1136-1142.

Yin, F. C., H. A. Spurgeon, H. L. Greene, E. G. Lakatta and M. L. Weisfeldt (1979). "Age-associated decrease in heart rate response to isoproterenol in dogs." Mech Ageing Dev 10(1-2): 17-25.

Zanesco, A. and E. Antunes (2007). "Effects of exercise training on the cardiovascular system: pharmacological approaches." Pharmacol Ther 114(3): 307-317.

Zhang, H. and J. E. Faber (2001). "Trophic effect of norepinephrine on arterial intimamedia and adventitia is augmented by injury and mediated by different alpha1adrenoceptor subtypes." Circ Res 89(9): 815-822. 
Zhang, Z. H., P. M. Dougherty and S. M. Oppenheimer (1999). "Monkey insular cortex neurons respond to baroreceptive and somatosensory convergent inputs." Neuroscience 94(2): 351-360. 


\section{Chapter 2}

\section{Coronary Artery Disease Affects Cortical Circuitry Associated with Brain-Heart Integration during Volitional Exercise}

(Published in Journal of Neurophysiology 114(2): 835-45, 2015)

\subsection{Introduction}

Coronary artery disease (CAD) increases risk of stroke, cognitive impairment and autonomic dysregulation (Martins, Hone et al. 2006, Zulli, Nicosia et al. 2008, Roberts, Knopman et al. 2010, Barekatain, Askarpour et al. 2014). In turn, impaired autonomic outcomes of CAD include diminished parasympathetic modulation of HR (Mancia, Cleroux et al. 1991, Seals, Taylor et al. 1994, Ford 1999). Moreover, adverse outcomes in autonomic cardiovascular control may exacerbate the disease pattern through tissue damage and diminished ability to affect rapid HR adjustments in response to stress and, thereby, limit the benefits that can be derived from exercise rehabilitation.

A role for the forebrain and brain stem in cardiac autonomic function has been established in both experimental studies in rodents (Cechetto and Saper 1990, Yasui, Breder et al. 1991) and clinical studies in patients with stroke or epileptic seizures in the prefrontal cortex (Cheung and Hachinski 2000). Recently, neuroimaging techniques have enabled investigation into a network of cortical regions associated with the autonomic nervous system and cardiovascular control in conscious humans (Critchley, Corfield et al. 2000, Gianaros, Van Der Veen et al. 2004, Williamson 2010, Basnayake, Green et al. 2012, Macey, Wu et al. 2012, Norton, Luchyshyn et al. 2013, Cechetto 2014, Shoemaker, Norton et al. 2014). These regions include the bilateral IC, ACC, posterior cingulate cortex (PCC), thalamus, MPFC, and HC. Importantly, the combined results indicate close homology between cortical sites identified experimentally in lower animals, and those observed in humans (Cechetto 2014). These experimental studies indicate that the IC, MPFC and HC are of particular relevance to HR control (Burns and Wyss 1985, Ruggiero, Mraovitch et al. 1987, Cechetto and Chen 1990, Yasui, Breder et al. 1991, 
Oppenheimer, Gelb et al. 1992, Verberne 1996, Fisk and Wyss 1997, Owens and Verberne 2001). Anatomically, the MPFC and HC have a large number of direct connections with subcortical structures (Verberne and Owens 1998) and have been linked in connectivity analyses of functional magnetic resonance imaging data in humans (Norton, Luchyshyn et al. 2013). Thus, the MPFC and HC form regions of interest for the current study.

Despite the mounting clinical evidence that vagal activity is an important predictor of cardiovascular prognosis in humans (Curtis and O'Keefe 2002), data are limited regarding the impact of CAD on the brain-heart connection. Volitional IHG contractions offer a unique opportunity to explore the cortical representation of autonomic cardiac control. More specifically, moderate intensity IHG exercise of short duration produces a rapid tachycardia in young and healthy individuals (Mancia, Iannos et al. 1978, Mark,

Victor et al. 1985, Wong, Masse et al. 2007) and pharmacologic evidence indicates that a decrease in parasympathetic dominance accounts for much of this rapid HR change (Hollander and Bouman 1975, Fagraeus and Linnarsson 1976, Mitchell, Reeves et al. 1989). In young individuals, the magnitude of this rapid increase in HR with IHG exercise is correlated with reduced activity within the MPFC (Gianaros, Van Der Veen et al. 2004, Wong, Masse et al. 2007) and the HC (Norton, Luchyshyn et al. 2013). It follows that these regions are associated with cardiovagal control.

The purpose of this study was to test the hypothesis that CAD impairs HR responses to volitional handgrip and that such impairment is related to dysregulation of the cortical autonomic network associated with HR control, particularly emphasizing activity patterns within the MPFC and HC.

\subsection{Methods}

\subsubsection{Participants}

A total of 40 individuals participated in this study. Observations were made in 17 patients with coronary artery disease (CAD) and 23 similarly-aged healthy control subjects (Control). Anthropometric and baseline cardiovascular data for each group are provided in Table 2.1. All subjects were non-smokers for longer than 10 years. Control subjects 
were free of any medications, and did not have diagnosed hypertension, vascular disease or diabetes. CAD patients were recruited from the London Health Sciences Centre for Cardiac Rehabilitation and Secondary Prevention Program following recent diagnosis of one of the following: admission for acute coronary syndrome (ST elevation or non ST elevation myocardial infarction), angina, per cutaneous coronary intervention, or coronary artery bypass graft. Thirteen of the patients were considered to be in functional Class I (as described by the New York Heart Association Functional Classification of heart failure), and four in functional Class II. Drug therapy included cholesterol lowering statins (94\%), beta-blockers (94\%), ACE-inhibitors/angiotensin II receptor blockers $(82 \%)$, calcium channel blockers (18\%), diuretics (6\%), and anti-platelets including aspirin (94\%). Patients were excluded if they had uncontrolled hypertension or a history of diabetes for more than 5 years. Both CAD patients and Controls were free of any neurological condition or disease. Each participant provided informed, written consent before participating in the study, which was approved by The University of Western Ontario Health Sciences Ethics Review Board and adhered to the Declaration of Helsinki.

\subsubsection{Experimental Design}

Participants completed two separate experimental sessions: 1) physiological recording (LAB session) and 2) a functional magnetic resonance neuroimaging session (fMRI; Robarts Research Institute Centre for Functional and Metabolic Imaging). The sessions were performed at the same time of day and separated by a minimum period of 1 week. Participants were familiarized with the experimental procedures prior to their first test session. Participants were instructed to arrive at the laboratory following a $12 \mathrm{~h}$ fast and to refrain from nicotine, alcohol, caffeine, and intense physical exertion for the same duration. Each session began with a maximal voluntary contraction (MVC) handgrip calibration, in which the participant was instructed to squeeze a non-magnetic handgrip device connected in series to a pressure transducer (Edwards Lifesciences, PX272, Irvine CA) to their maximal ability while in the supine position. This was repeated twice with the larger value calibrated as $100 \%$. Isometric handgrip (IHG) exercise was performed with the right hand in all subjects, regardless of handedness ( $n=37$ right-handed). During each recording session, visual feedback was provided to the participant of their achieved 
force in real-time. Baseline data were collected over 5 min of quiet supine rest. Four repeated bouts each of 30\%, $40 \%$ and 50\% of MVC force (LAB session), and seven repeated bouts of IHG at $40 \%$ MVC force (fMRI session) were performed, with each contraction lasting $20 \mathrm{sec}$ and separated by $40 \mathrm{sec}$ of rest. The number of trials was increased in the fMRI session to increase the signal-to-noise ratio. The level of perceived exertion produced by the exercise was monitored after each trial on a scale from 6-20 (Borg 1982).

\subsubsection{Cardiorespiratory Fitness Test}

Breath-by-breath measurements of oxygen consumption $\left(\mathrm{VO}_{2}\right), \mathrm{HR}$ and $\mathrm{BP}$ were recorded throughout the test. Maximal oxygen consumption $\left(\mathrm{VO}_{2 \max }\right)$ is an established marker of cardiorespiratory fitness and a clinically accepted surrogate marker for left

ventricular function (Fletcher, Balady et al. 2001). Each subject's $V O_{2 \max }$ was estimated from a graded treadmill exercise test to volitional exhaustion under standard clinical observation (ACSM 1995).

\subsubsection{Physiological Data Analysis}

Analog signals were sampled at $1000 \mathrm{~Hz}$ with an on-line data acquisition and analysis system (PowerLab, ADInstruments, Mountain View, CA, USA). HR was calculated from successive R-R intervals obtained from the ECG signal. BP from the Finometer was converted to mean arterial pressure (MAP) using the formula MAP $=1 / 3 \mathrm{SBP}+2 / 3$ DBP. Beat-by-beat HR data were averaged over $2.5 \mathrm{~s}$ bins (the TR interval for functional scans) and time aligned to ensure a corresponding mean value for each functional scan obtained during the fMRI collection period. The HR response $(\Delta \mathrm{HR})$ to the IHG was determined by averaging the response over the last 10s of each rest and IHG interval. HR responses for each participant were averaged over the four repeated blocks in the three separate trials $(30 \%, 40 \%$ and $50 \%)$.

The effect of group and IHG intensity on HR response was assessed using a two-way mixed ANOVA with an alpha level of $\mathrm{p}<0.05$. Statistical analyses were performed using SigmaPlot (version 12.5, 2011). The Shapiro-Wilk test for normal distribution, as well as 
the Holm-Sidak method for pairwise multiple comparisons were used. All data are presented as mean \pm standard deviation.

\subsubsection{Neuroimaging Recording Session}

All imaging data were collected using a whole body 3-Tesla imaging system (Magnetom Prisma, Siemens Medical Solutions, Erlangen, Germany) with a 32-channel head coil (Barberi, Gati et al. 2000). A high-resolution $\mathrm{T}_{1}$-weighted structural volume was acquired with a 3D MPRAGE sequence at the beginning of the scanning session (sagittal, matrix 256 X $240 \mathrm{~mm}$, voxel resolution 1.0 X 1.0 X $1.0 \mathrm{~mm}, 1 \mathrm{~mm}$ slice thickness, no gap, flip angle $9^{\circ}, \mathrm{TE}=2.98 \mathrm{~ms}, \mathrm{TI}=900 \mathrm{~ms}, \mathrm{TR}=2.3 \mathrm{~ms}$ ). Transmission and detection of the BOLD contrast signal were acquired by $\mathrm{T}_{2}$-weighted gradient echo-echo planar imaging pulse sequence with the following parameters: TE $=30 \mathrm{~ms}$; FOV $=240 \times 240 \mathrm{~mm}$, flip angle $=90^{\circ}$. Forty-five interleaved axial slices $(3.0 \times 3.0 \mathrm{~mm}$ in-plane voxel resolution, $\mathrm{TR}=2.5 \mathrm{~s}$ ) were acquired in each volume. Five volumes were acquired in the resting participant prior to actual data collection to allow for magnetization equilibrium; these were discarded prior to data analysis. Head movement was limited during the experimental session within a head cradle packed with foam padding, and each subject was instructed to avoid head movements during the scanning period. Beat-by-beat HR was calculated from the continuous signal derived from an MRI-compatible pulse Oximeter (Nonin Medical Inc, 8600FO MRI, Plymouth, MN) placed over the index finger of the non-exercising left hand. In each session, analog signals for pulse recordings and IHG contraction force were sampled at $1000 \mathrm{~Hz}$ with an on-line data acquisition and analysis system (PowerLab, ADInstruments, Mountain View, CA, USA). Respiratory frequency was monitored continuously during the exercise period.

\subsubsection{Neuroimaging Data Analysis}

The HR response $(\Delta H R)$ to the handgrip was determined by averaging the response over the last 10 s of each rest and IHG interval. Individual HR time courses were determined using $2.5 \mathrm{~s}$ averages of the beat-by-beat HR measures to generate time-aligned data with the BOLD imaging acquisition. For both the $\Delta \mathrm{HR}$ and the HR time course, responses for each participant were averaged over the seven repeated blocks at $40 \%$ MVC. 
All fMRI data were analyzed using Brain Voyager QX 2.8.2 (Brain Innovation, Maastricht, Netherlands) (Goebel, Esposito et al. 2006). At the first (individual) level, preprocessing included interscan slice acquisition time correction, linear trend removal, temporal high-pass filtering to remove low-frequency drifts, and rigid-body transformation of data to the first acquired image to correct for motion. Individual functional data were co-registered to their respective anatomical template, and subsequently transformed to Talairach space (Talairach and Tournoux 1988). The change in BOLD signal over the exercise period was modeled with a boxcar function convolved with a canonical haemodynamic response function and regressed with the individual movement parameters generated during preprocessing. This resulted in subject-specific contrast images containing whole brain information related to sites of both increased and decreased BOLD signal, relative to baseline, during the IHG task as a function of the task itself and the individual HR correlation. The General Linear Model was used to calculate the parameter estimates for all brain voxels (Friston, Holmes et al. 1995).

To make valid population inferences, a second-level, two-group, random effects (RFX) analysis was performed both in response to the task and the HR regression to assess the consistency of effects between individuals based on the variability of the first-level estimates across subjects. Subsequently, a subtraction analysis of the group mean parameter estimates was performed to assess significant differences between Control and CAD groups. Corrections for multiple comparisons were made using the false discovery rate $(\mathrm{p}<0.05)$, as well as cluster level threshold estimation (Hagler, Saygin et al. 2006), with 1000 iterations of Monte Carlo simulation and a statistical threshold of $p<0.05$ for the main task effects. Due to the abundance of neural activity, both corrections were performed sequentially, such that the final results represent only clusters > 10 voxels in size (unless otherwise specified). Based on earlier data in young individuals performing the same IHG protocol (Wong, Masse et al. 2007, Norton, Luchyshyn et al. 2013), an $a$ priori region-of-interest analysis was performed for relevant cortical autonomic network regions including the IC, ACC, PCC, thalamus, MPFC, and HC. All fMRI data are represented in radiologic convention (i.e. subject's right appears on the left). 
Probabilistic functional maps were created for each group to investigate the spatial consistency of activation patterns across subjects. These maps represent the relative number of subjects leading to significant task activity at each spatial location.

\subsection{Results}

\subsubsection{Physiological Responses}

The groups were not statistically different in age, mean arterial pressure, resting HR or maximal handgrip strength (Table 2.1). The heart rate response ( $\triangle \mathrm{HR}$ ) to the $40 \% \mathrm{MVC}$ contraction was the same during the physiological (LAB) and neuroimaging (fMRI) session in both groups (Table 2.1). There was a significant effect of group $(\mathrm{p}=0.03)$ on $\Delta \mathrm{HR}$ across all IHG tasks (Figure 2.1). None of the participants reported feeling any significant degree of aversive emotional stress or forearm fatigue as indicated by the Borg scale outcomes (Table 2.1)(Borg 1982). Further, the $\Delta \mathrm{HR}$ at maximal exertion (stress test) in Control participants was greater than CAD patients (Table 2.1, $\mathrm{p}<0.001$ ). All patients exercised to maximum effort (Control: $19 \pm 1$, CAD: $19 \pm 1$ on 6-20 Borg Scale) and the tests were not discontinued due to medical reasons (angina, ST depression, arrhythmia or abnormal BP response). In addition, left ventricular ejection fraction (LVEF) was normal $(>=50 \%)$ in $11 / 17$ patients, mild $(35-49 \%)$ in $3 / 17$ patients, moderate (20-34\%) in 2/17 patients, and severe $(<20 \%)$ in one patient.

\subsubsection{Functional (BOLD) Imaging Data: First-level (Individual) Response to $40 \%$ IHG Task}

A-priori region-of-interest analysis revealed high inter-subject variability in both groups, with bilateral IC activation observed in 22/23 Control subjects and 15/17 CAD patients, ACC deactivation observed in 17/23 Control and 14/17 CAD, PCC deactivation observed in 18/23 Control and 16/17 CAD, thalamus activation observed in 20/23 Control and 7/17 CAD, MPFC deactivation observed in 16/23 Control and 12/17 CAD, and HC deactivation observed in 8/23 Control and 8/17 CAD ( $\mathrm{p}<0.05$, FDR). 


\subsubsection{Functional (BOLD) Imaging Data: First-level (Individual) Response Correlated with Heart Rate}

Bilateral IC activation was observed in 18/23 Control subjects and 13/17 CAD patients, ACC deactivation was observed in 11/23 Control and 5/17 CAD, PCC deactivation was observed in 5/23 Control and 2/17 CAD, thalamus activation was observed in 16/23 Control and 11/17 CAD, MPFC deactivation was observed in 16/23 Control and 12/17 CAD, and HC deactivation was observed in 2/23 Control and 2/17 CAD ( $\mathrm{p}<0.05$, FDR).

\subsubsection{Functional (BOLD) Imaging Data: Second-level (Group) Response to $40 \%$ IHG Task}

A-priori region-of-interest analysis revealed common increases in BOLD signal in the primary motor cortex (precentral cortex), bilateral anterior IC and occipital lobe (Tables 2.2 and 2.3; Figure 2.2). In addition, common deactivation was observed in the PCC $(\mathrm{p}<0.05$, FDR; Tables 2.2 and 2.3, Figure 2.2). In Control subjects, activation was observed in the ACC and thalamus; and deactivation was observed in the HC. No signal change was observed in the $\mathrm{HC}$ of the $\mathrm{CAD}$ group. No signal change was observed in the MPFC in either Control or CAD patients at the group level.

\subsubsection{Contrasting BOLD Responses between Control and CAD to 40\% IHG Task}

Comparisons of activated regions between Control and CAD during the $40 \%$ IHG task are shown in Figure 2.3. In subtraction analyses for CAD>Control, greater activation (or less deactivation) was observed in the PCC and MPFC. The contrast CAD $<$ Control showed activation in the right anterior insula, bilateral precentral cortex, and occipital lobe $(\mathrm{p}<0.05)$.

\subsubsection{Functional (BOLD) Imaging Data: Second-level (Group) Response Correlated with Heart Rate}

Extensive activation patterns were revealed in both Control and CAD groups, but lacked significant deactivation in expected autonomic regions (Figure 2.4). Specifically, the bilateral IC and precentral gyrus were activated in both Control and CAD groups while deactivation in the MPFC and HC were absent (FDR p<0.05). 


\subsubsection{Contrasting BOLD Responses between Control and CAD Correlated with Heart Rate}

Comparisons of activated regions between Control and CAD during the $40 \%$ IHG task correlated with the individual HR time courses are shown in Figure 2.5. In subtraction analyses for $\mathrm{CAD}>\mathrm{Control}$, greater activation was observed in the perigenual anterior cingulate cortex. The contrast $\mathrm{CAD}<\mathrm{Control}$ demonstrated activation in the bilateral insula and posterior cingulate cortex $(\mathrm{p}<0.05)$.

\subsubsection{Probability Mapping}

Probabilistic maps were created for Control and CAD groups to provide a general means to evaluate the spatial consistency of task-specific brain activation across subjects. We plotted the cross-subject (Control and CAD) overlap probability maps for $40 \%$ IHG at a range of $0-100 \%$ (Figure 2.6). Control subjects (yellow/orange) displayed greater anatomical consistency compared to CAD patients (blue) who showed much greater variability in activation responses (cluster threshold=15 voxels). Probability percent values (overlap) between Control and CAD in expected CAN regions include: the left anterior IC (8.43\% overlap; x, y, z coordinates: $-40,14,5)$, right anterior IC $(11.02 \%$; , y, z coordinates: $34,20,9)$, PCC $(0.08 \%$; x, y, z coordinates: $-8,-55,14)$, precentral gyrus $(31.97 \%$; $\mathrm{x}, \mathrm{y}, \mathrm{z}$ coordinates: $34,-21,52)$, and MPFC (2.81\%; $\mathrm{x}, \mathrm{y}, \mathrm{z}$ coordinates: $4,37,-3)$. 
Table 2.1 Anthropometric and baseline cardiovascular data during baseline and isometric handgrip exercise (mean $\pm \mathrm{SD}$ ).

\begin{tabular}{cccccccccc}
\hline Group & Age & Sex & $\begin{array}{c}\text { MAP } \\
(\mathbf{m m H g})\end{array}$ & $\begin{array}{c}\text { MVC } \\
(\mathbf{m V})\end{array}$ & $\begin{array}{c}\text { Resting } \\
\mathbf{H R} \\
(\mathbf{b p m})\end{array}$ & $\begin{array}{c}\mathbf{4 0 \%} \\
\mathbf{\Delta H R}\end{array}$ & $\begin{array}{c}\mathbf{4 0 \%} \\
(\mathbf{L A B})\end{array}$ & $\begin{array}{c}\mathbf{4 0 \%} \\
(\mathbf{f M R I})\end{array}$ & $\begin{array}{c}\text { Stress } \\
\text { RPE } \\
\text { Test } \Delta \mathbf{H R} \\
(\mathbf{L A B})\end{array}$ \\
\hline $\begin{array}{c}\mathrm{CTRL} \\
(\mathrm{n}=23)\end{array}$ & $63 \pm 11$ & $15 \mathrm{M}, 8 \mathrm{~F}$ & $90 \pm 9$ & $62 \pm 28$ & $58 \pm 8$ & $4 \pm 2$ & $2 \pm 2$ & $13 \pm 2$ & $105 \pm 16$ \\
$\begin{array}{c}\mathrm{CAD} \\
(\mathrm{n}=17)\end{array}$ & $59 \pm 9$ & $13 \mathrm{M}, 4 \mathrm{~F}$ & $87 \pm 10$ & $63 \pm 31$ & $59 \pm 5$ & $3 \pm 2$ & $3 \pm 2$ & $11 \pm 2^{*}$ & $78 \pm 24^{*}$
\end{tabular}

CTRL, healthy older controls; CAD, coronary artery disease patients; MAP, mean arterial pressure; MVC, maximal voluntary contraction (average of LAB + fMRI sessions); HR, heart rate (beats/min); fMRI, neuroimaging session; LAB, physiological recording session; Stress test, voluntary maximal exertion; Borg rate of perceived exertion (RPE) scale: 6-20. RPE=11, "light" exercise; 13, "somewhat hard". There was a main effect of group such that CAD patients had less of a HR response during all conditions than Control. "Different from Control $(\mathrm{p}<0.05)$. 
Table 2.2 BOLD signal changes to $40 \%$ MVC handgrip in Control subjects.

\begin{tabular}{lccccccc}
\hline \multicolumn{1}{c}{ Location } & & Side & \multicolumn{3}{c}{ Coordinates } & T-score & Number of \\
\cline { 4 - 6 } & & & $\boldsymbol{x}$ & $\boldsymbol{y}$ & $\boldsymbol{z}$ & & Voxels \\
\hline Insula & $(\uparrow)$ & $\mathrm{L}$ & -38 & 22 & 9 & 4.39 & 986 \\
Insula & $(\uparrow)$ & $\mathrm{R}$ & 31 & 23 & 9 & 5.44 & 1000 \\
Dorsal ACC & $(\uparrow)$ & $\mathrm{R}$ & 5 & 40 & 14 & 2.93 & 398 \\
Mid-superior CC & $(\uparrow)$ & $\mathrm{R}$ & 1 & -1 & 30 & 3.78 & 766 \\
PCC & $(\downarrow)$ & $\mathrm{R}$ & 3 & -50 & 20 & -3.05 & 363 \\
Precentral gyrus & $(\uparrow)$ & $\mathrm{L}$ & -31 & -6 & 50 & 4.03 & 809 \\
Precentral gyrus & $(\uparrow)$ & $\mathrm{R}$ & 31 & -6 & 50 & 6.24 & 1000 \\
Postcentral gyrus & $(\uparrow)$ & $\mathrm{R}$ & 31 & -27 & 50 & 6.61 & 1000 \\
Thalamus & $(\uparrow)$ & $\mathrm{L}$ & -9 & -17 & 12 & 4.18 & 935 \\
Thalamus & $(\uparrow)$ & $\mathrm{R}$ & 8 & -17 & 12 & 4.61 & 896 \\
Hippocampus & $(\downarrow)$ & $\mathrm{L}$ & -28 & -27 & -3 & -3.26 & 655 \\
Occipital & $(\uparrow)$ & $\mathrm{R}$ & 0 & -87 & 9 & 4.46 & 823 \\
\hline
\end{tabular}

ACC $=$ anterior cingulate cortex, PCC $=$ posterior cingulate cortex. $(\uparrow)=$ activation; $(\downarrow)=$ deactivation. (Talairach coordinates represent voxel of maximum response: $x$ represents position in brain on horizontal axis, $y$ represents position on vertical axis, $z$ represents the depth position). 
Table 2.3 BOLD signal changes to 40\% MVC handgrip in CAD subjects.

\begin{tabular}{|c|c|c|c|c|c|c|c|}
\hline \multirow[t]{2}{*}{ Location } & & \multirow[t]{2}{*}{ Side } & \multicolumn{3}{|c|}{ Coordinates } & \multirow[t]{2}{*}{ T-score } & \multirow{2}{*}{$\begin{array}{c}\text { Number of } \\
\text { Voxels }\end{array}$} \\
\hline & & & $x$ & $y$ & $z$ & & \\
\hline Insula & $(\uparrow)$ & $\mathrm{L}$ & -33 & 18 & 11 & 4.40 & 236 \\
\hline Insula & $(\uparrow)$ & $\mathrm{R}$ & 34 & 13 & 11 & 4.33 & 313 \\
\hline Mid-superior CC & $(\downarrow)$ & $\mathrm{L}$ & -8 & -32 & 33 & -4.29 & 532 \\
\hline $\mathrm{PCC}$ & $(\downarrow)$ & $\mathrm{L}$ & -8 & -48 & 11 & -4.22 & 362 \\
\hline Precentral gyrus & $(\uparrow)$ & $\mathrm{R}$ & 34 & -23 & 44 & 5.29 & 525 \\
\hline Precentral gyrus & $(\uparrow)$ & $\mathrm{L}$ & -46 & 1 & 33 & 4.04 & 750 \\
\hline Occipital & $(\uparrow)$ & $\mathrm{R}$ & 0 & -87 & 9 & 4.06 & 792 \\
\hline
\end{tabular}

ACC $=$ anterior cingulate cortex, PCC $=$ posterior cingulate cortex. $(\uparrow)=$ activation; $(\downarrow)=$ deactivation. (Talairach coordinates represent voxel of maximum response: $x$ represents position in brain on horizontal axis, $y$ represents position on vertical axis, $z$ represents the depth position). 
Figure 2.1 Graded heart rate response to isometric handgrip.
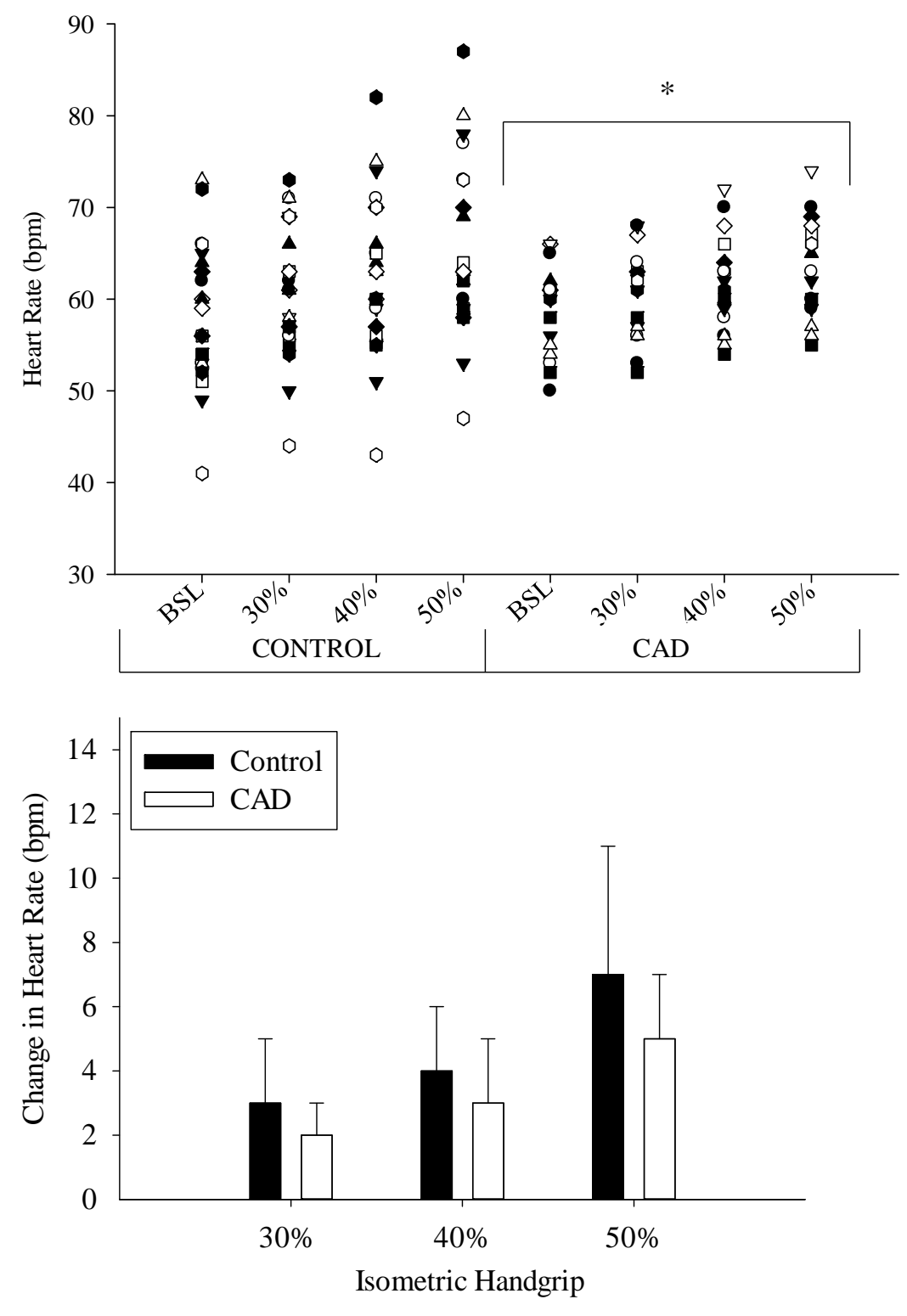

Control and coronary artery disease patients (CAD) represented as individuals (top panel) and as a group (bottom panel). BSL=Baseline. *Different from Control; main effect of Group ( $\mathrm{p}<0.05)$. 
Figure 2.2 Cortical functional response to $40 \%$ IHG task in Control (CTRL; top three images) and CAD (lower three images).

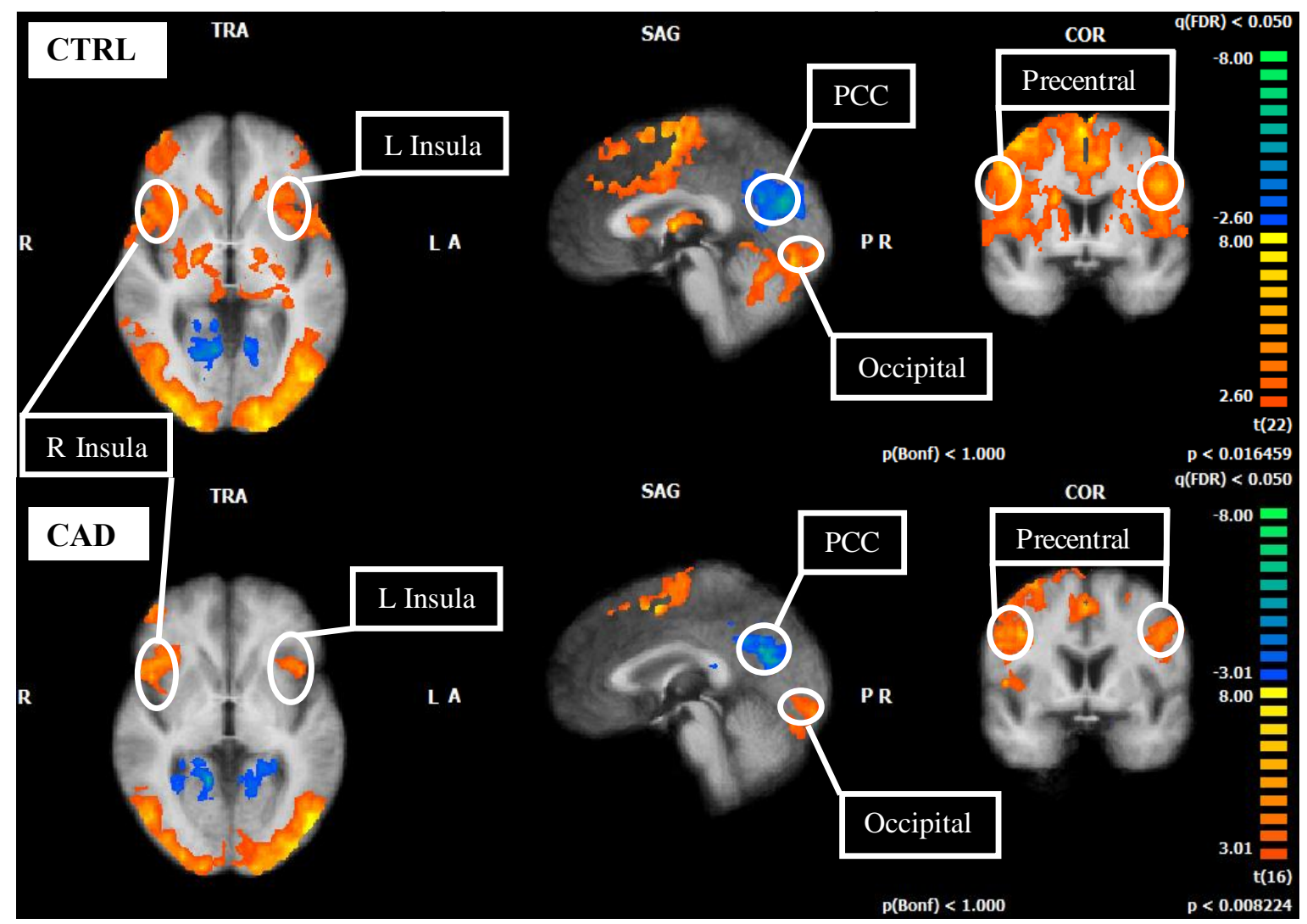

L: left, R: right, PCC: posterior cingulate cortex. T-statistics (beta values) at specified regions (Talairach coordinates, circled) are represented as an average for each group. FDR, $\mathrm{p}<0.05$, corrected for multiple comparisons. Color scheme identified by scale at right. Red/warm colors denote regions of activation above baseline levels, blue/cold colors denote regions of deactivation below baseline levels (exact values given in beta graphs, Figure 2.3). Note the absence of deactivation at the medial prefrontal cortex and hippocampus in both groups. 
Figure 2.3 Subtraction Result for Group 1 average (CAD) vs Group 2 average (Control) to $40 \%$ IHG boxcar analysis.
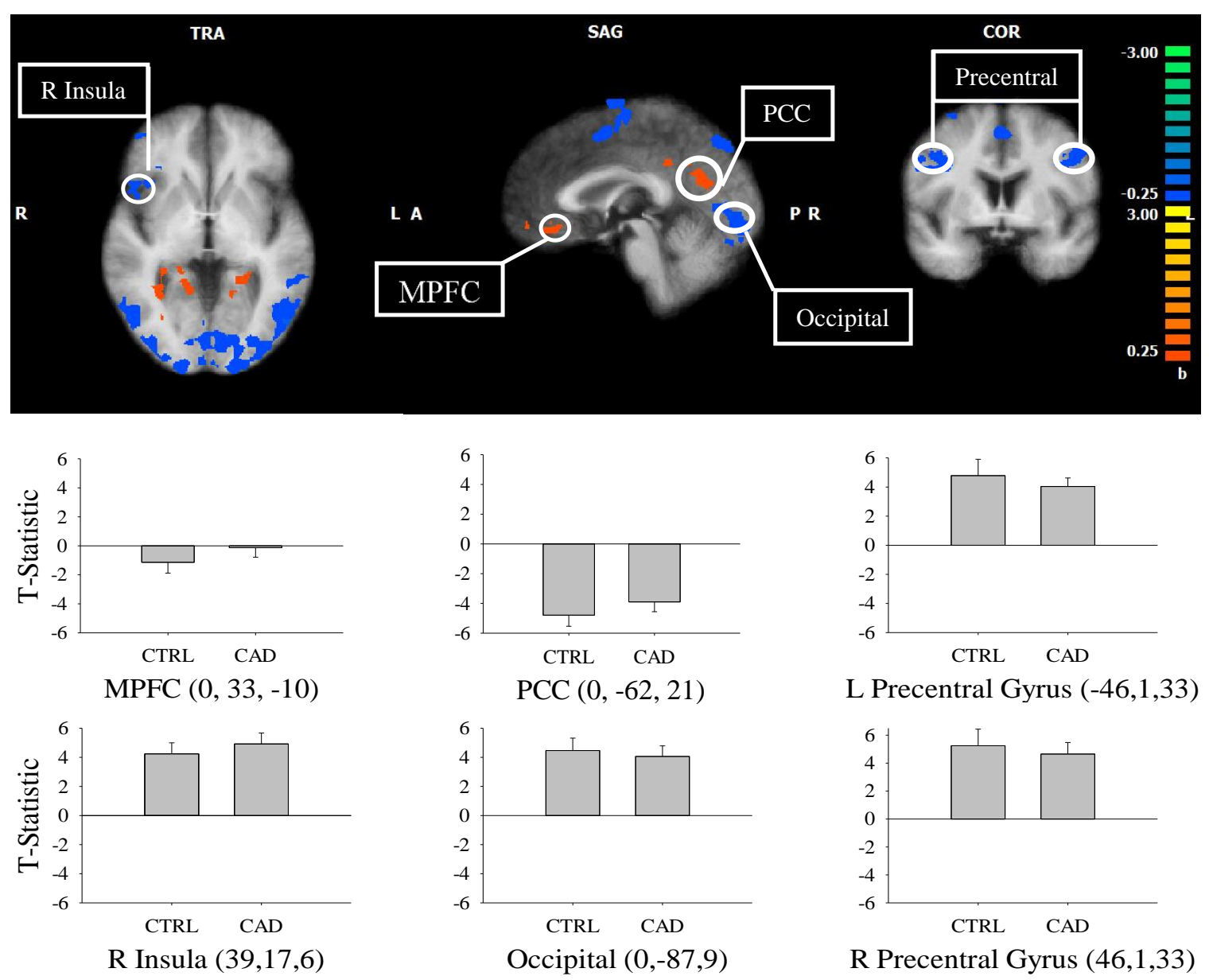

L Precentral Gyrus $(-46,1,33)$

L: left, R: right, MPFC: medial prefrontal cortex, PCC: posterior cingulate cortex. Warm colors show areas where there is a positive difference with respect to group 1 (CAD > Control), and cold colors show areas where there is a negative difference with respect to group 1 (CAD < Control). T-statistic (beta value) at specified regions (Talairach coordinates, circled) are represented as an average for each group and denoted by color scale at right, threshold $=2 ; \mathrm{p}<0.05$. Cluster threshold $=10$ voxels. Error bars represent standard deviation. 
Figure 2.4 Cortical functional response correlated to individual heart rate time course during 40\% IHG task in Control (CTRL; top three images) and CAD (lower three images).

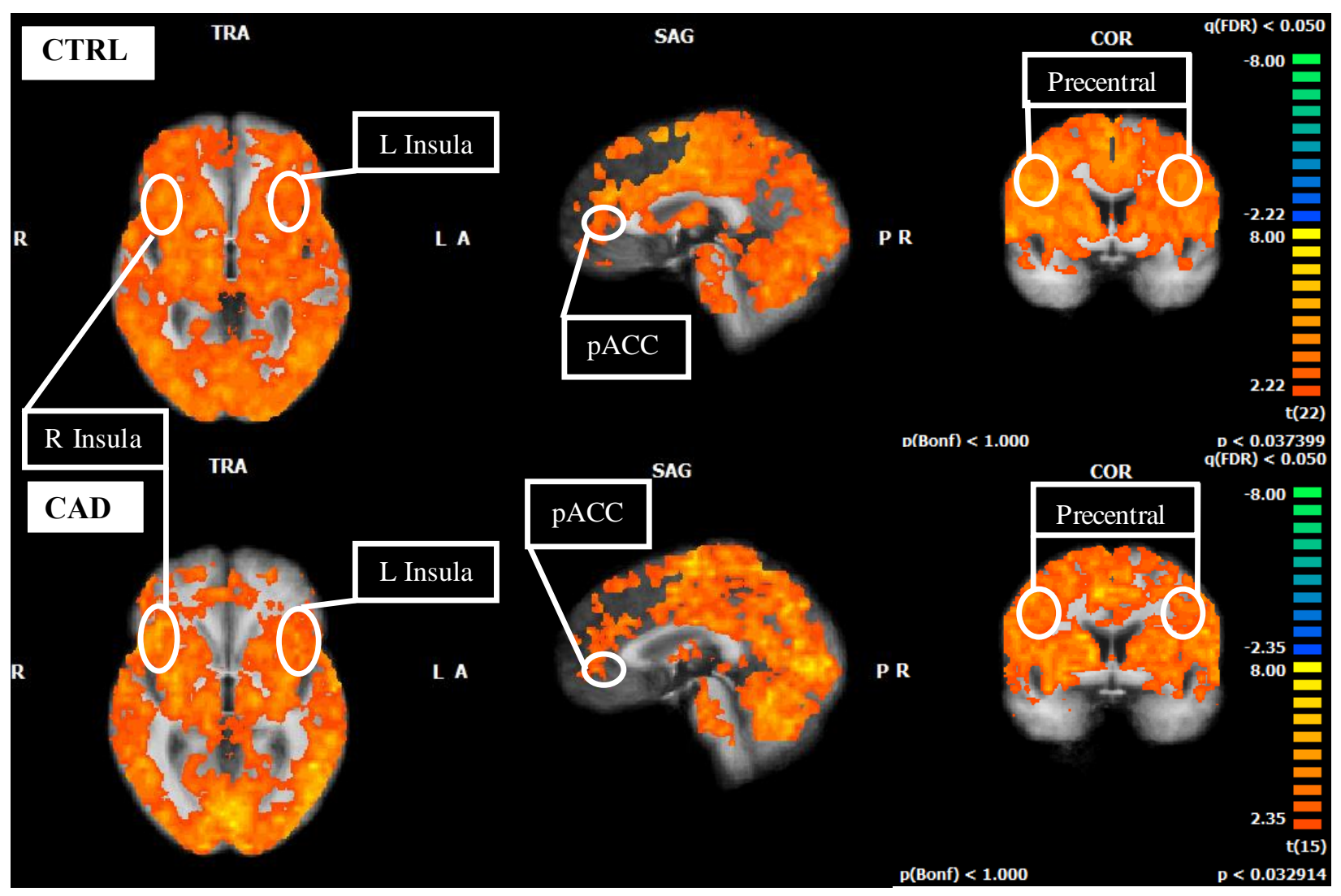

L: left, R: right, pACC: perigenual anterior cingulate cortex. T-statistics (beta values) at specified regions (Talairach coordinates given, circled) are represented as an average for each group. FDR, $\mathrm{p}<0.05$, corrected for multiple comparisons. Color scheme identified by scale at right. Red/warm colors denote regions of activation above baseline levels, blue/cold colors denote regions of deactivation below baseline levels (exact values given in beta graphs, Figure 2.5). Note the absence of deactivation at the medial prefrontal cortex and hippocampus in both groups. 
Figure 2.5 Subtraction result for Group 1 average (CAD) vs Group 2 average (Control) correlated to individual heart rate time course during $40 \%$ IHG task.

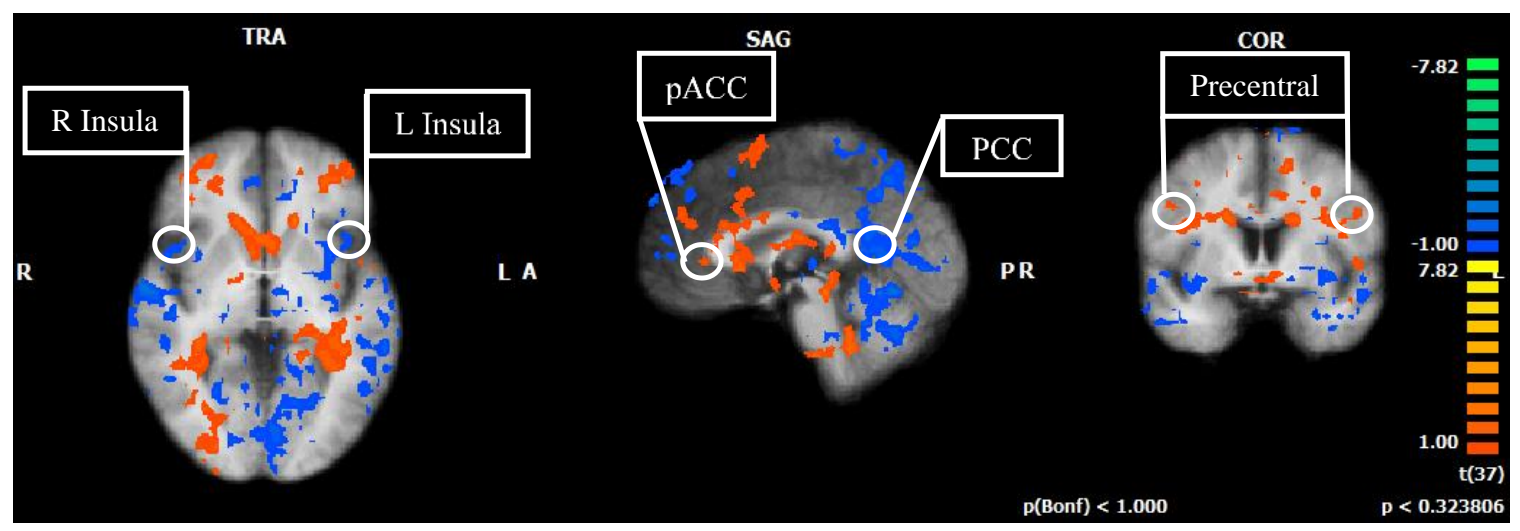

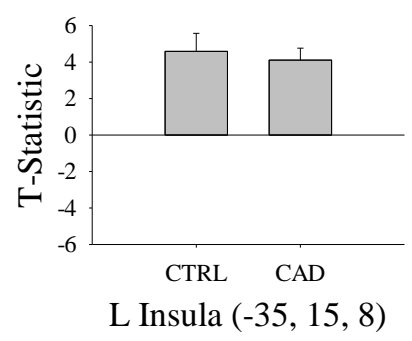

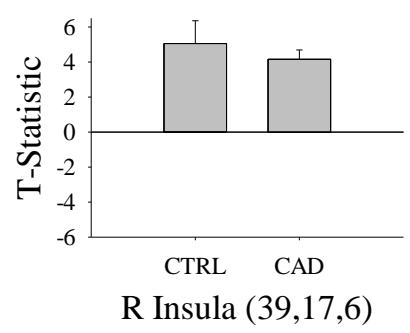

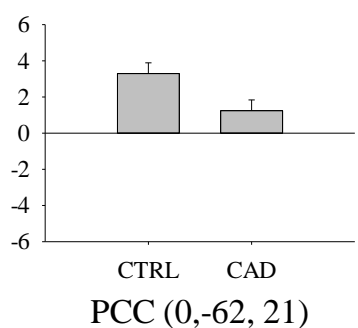

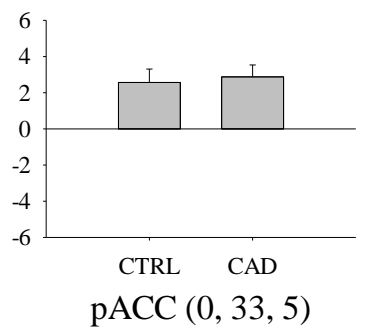

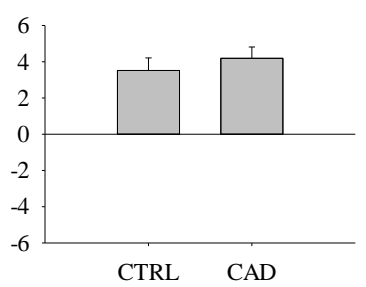

L Precentral Gyrus $(-46,1,33)$

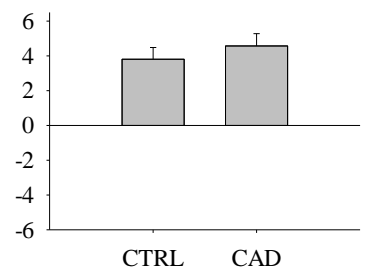

R Precentral Gyrus $(46,1,33)$

L: left, R: right, PCC: posterior cingulate cortex; pACC: perigenual anterior cingulate cortex. Warm colors show areas where there is a positive difference with respect to group 1 (CAD > Control), and cold colors show areas where there is a negative difference with respect to group 1 $(\mathrm{CAD}<\mathrm{Control})$. T-statistic (beta value) at specified regions (Talairach coordinates, circled) are represented as an average for each group and denoted by color scale at right, threshold $=2$; $\mathrm{p}<0.05$. Cluster threshold $=10$ voxels. Error bars represent standard deviation. 
Figure 2.6 Probability Mapping.

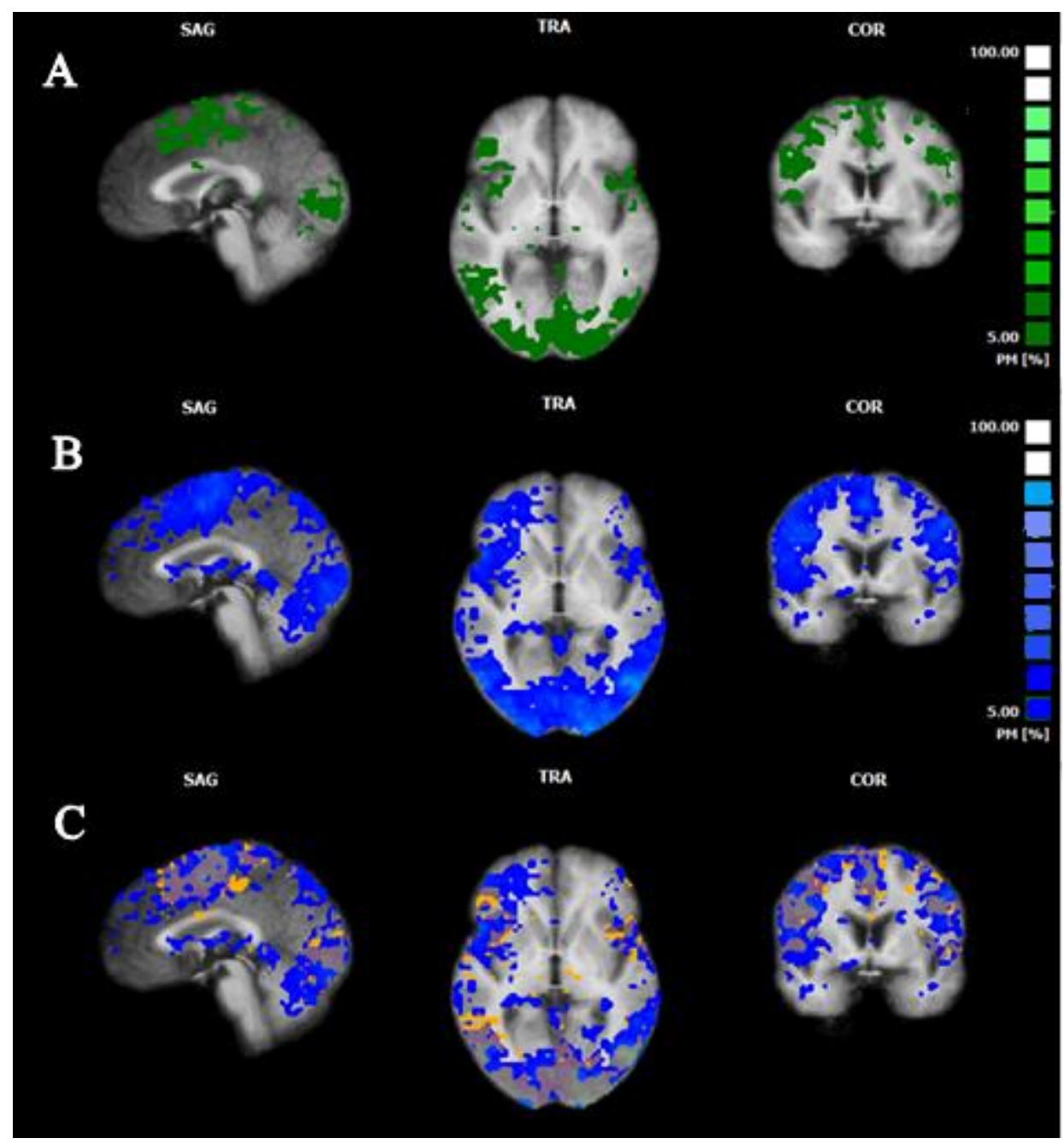

(A) Control subjects (green), 0\% minimum threshold. (B) Coronary artery disease patients (CAD; blue), 5\% minimum threshold. Each colored cluster represents the relative percentage of subjects leading to significant task activity during a $40 \% \mathrm{MVC}$ handgrip task based on the bar graph at right. (C) Probability map overlap of both groups. Control $=$ orange CAD $=$ blue (voxel threshold $=15$ voxels; $0 \%$ minimum threshold). CAD patients (blue) had much greater variability in activation responses than Control who indicated greater anatomical consistency. 
Figure 2.7
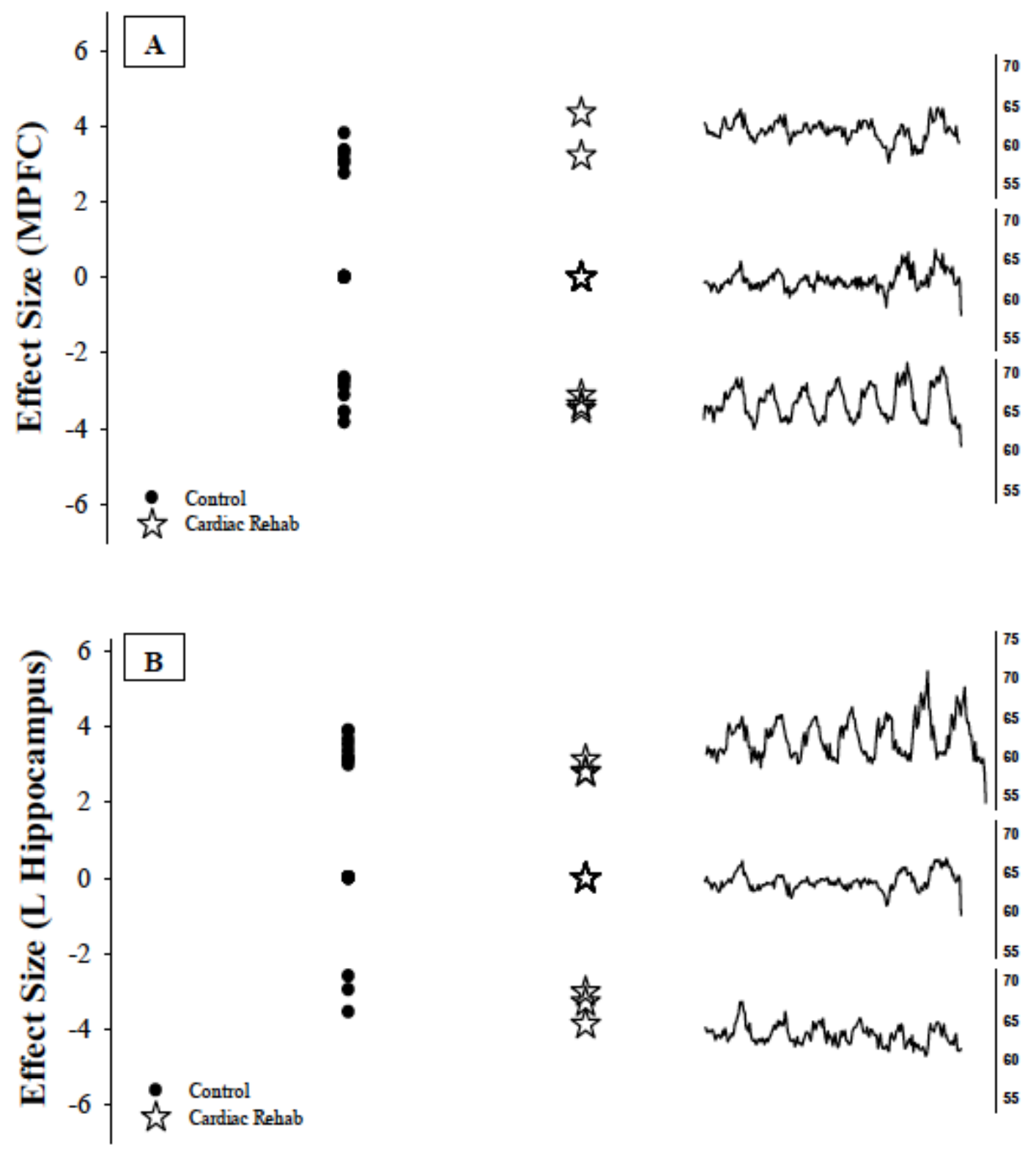

The effect size (left) in a priori regions (panel A: medial prefrontal cortex; panel B: left hippocampus) to the $40 \%$ isometric handgrip task with the average heart rate response (bpm) of those individuals (right). Marked individual variability was observed in neural and heart rate responses in both groups. 


\subsection{Discussion}

In contrast to healthy Controls, the CAD patient group demonstrated diminished $\mathrm{HR}$ responses across all exercise workloads and high variance in activation patterns amongst regions of the cortical autonomic network. These cortical patterns appear to be consistent with the overall suppressed HR response despite the ability to perform the IHG task adequately. Therefore, the current data supports the hypothesis that CAD alters the cortical circuitry associated with exercise and patients exhibit accelerated age-related dysregulation of the brain-heart connection.

An initial important observation of the current study was the difference in HR responses to IHG between the current participants and those of younger individuals reported earlier from our laboratory (Wong, Masse et al. 2007, Goswami, Frances et al. 2012, Norton, Luchyshyn et al. 2013). This difference was statistically significant, as determined by an independent group's t-test that contrasted the present data with those published earlier. Specifically, young individuals ( $25 \pm 4$ years), when compared to the current participants (61 \pm 10 years), generate a much larger HR response $(6-15 \mathrm{bpm})$ to a similar relative IHG tension $(\mathrm{p}<0.0001)$. Mechanistically, this IHG protocol is designed to engage exerciseonset reflexive increases in $\mathrm{HR}$ that predominantly reflect reduced dominance of parasympathetic control (Hollander and Bouman 1975, Fagraeus and Linnarsson 1976, Mitchell, Reeves et al. 1989). Therefore, the smaller HR responses in Control, and smaller yet in CAD, are likely a consequence of age-related impairment of parasympathetic outflow (Seals, Taylor et al. 1994, Monahan, Dinenno et al. 2001) that is further negatively impacted by CAD (Gribbin, Pickering et al. 1969, Eckberg, Drabinsky et al. 1971). Recently, our laboratory reported the cortical activation patterns and HR responses in healthy individuals ranging from 21-80 years of age, with conclusions that age alone does not determine a smaller $\Delta H R$ response (Norton, Luchyshyn et al. 2013) in that several older adults still generated similar responses to young individuals. Thus, inter-individual variability in HR responses was augmented with increasing age. The current study further supports a depressed HR response overall, as well as enhanced variability in HR responses, as an effect of age (Figure 2.1). 
A second observation of the current study was the marked and unexpected differences in brain activation patterns associated with both the IHG task and the HR response in both the Control and CAD patients compared to previous studies from our laboratory performed by young and healthy individuals (Wong, Masse et al. 2007, Goswami, Frances et al. 2012, Norton, Luchyshyn et al. 2013). Specifically, the current participants exhibited a large and widespread pattern of enhanced brain activation relative to baseline when correlated with both the IHG task and the HR response. This widespread activation pattern was different from the discrete pattern observed in young individuals specifically in regions related to autonomic control. Within this pattern of higher overall activation, however, there was a marked absence of deactivation within the MPFC and HC in the current participants (at least at the group level) which are consistently present in young healthy subjects (Wong, Masse et al. 2007, Goswami, Frances et al. 2012, Norton, Luchyshyn et al. 2013). Yet, group-level activation was observed in the bilateral insula, and deactivation was observed in the posterior cingulate cortex, observations that are consistent with previously published results in young subjects.

Although this apparent "overactivation" identified above is unique in the context of cardiovascular control, it has been reported earlier in the context of perceptual or cognitive tasks performed by aged individuals. The mechanism(s) of these patterns is yet unknown. They may reflect alterations in the coupling between regional blood flow and oxygen extraction. However, some hypothesize these patterns to reflect compensatory neural responses (Reuter-Lorenz 2002, Cabeza, Daselaar et al. 2004) where, in the aging brain, previously connected networks are disrupted such that alternative patterns emerge which must "work harder" to make up either for its own declining efficiency or for processing deficiencies elsewhere in the brain. The current observations appear to be the first to report a similar phenomenon related to volitional tasks such as IHG exercise. If this observation reflects neural compensation, the smaller response in CAD versus Control participants becomes the third important observation of the current study (Figure 2.4). The degree of compensatory activation is thought to be related to a sense of effort required to perform the task (Reuter-Lorenz and Park 2010). In this context, the smaller amount of compensation in $\mathrm{CAD}$ patients may reflect lower perceived effort required to perform the IHG. However, the similar Borg scores of perceived effort and identical 
absolute and relative workloads produced (MVC, Table 2.1) argue that the smaller brain activation patterns in CAD patients are not related to perceived effort. Previously, our laboratory reported accelerated age-related cortical atrophy in CAD patients (Anazodo, Shoemaker et al. 2013). Thus, it may be that age-related compensatory responses to IHG are also related to accelerated brain atrophy and/or impaired local flow-metabolism coupling.

The probability mapping analysis indicated that CAD patients exhibited greater regional variability in activation responses to the $40 \%$ IHG task than Control. Probability percent values, which reflect overlapping patterns of activation between Control and CAD participants, were highest in the primary motor cortex (31.97\%), but significant variability existed in expected CAN regions such as the left anterior IC (8.43\%), right anterior IC $(11.02 \%)$, PCC $(0.08 \%)$, and MPFC (2.81\%). Thus, it appears that brain regions required for motor activity are retained in $\mathrm{CAD}$, but that those regions believed to be required for explicit autonomic homeostatic functions, such as the MPFC and HC, are dysregulated more in CAD patients.

A notable outcome of the current study was the absence of deactivation associated with the HR response to IHG in the MPFC and HC, in both Control and CAD patients when studied at the group level (Figure 2.4). The MPFC region was of particular interest in the present study as it has been associated with cardiovagal control across many stimuli that elicit cardiovascular arousal (Critchley, Corfield et al. 2000, Williamson, McColl et al. 2003, Gianaros, Van Der Veen et al. 2004, Matthews, Paulus et al. 2004, Resstel, Fernandes et al. 2004, Kimmerly, O'Leary et al. 2005, Thayer, Sollers et al. 2009, Norton, Luchyshyn et al. 2013). Previous studies have further suggested that the MPFC is involved in the processes of integrating sensory information during the resting default state and its activity is hence attenuated during goal-directed behaviours (Raichle, MacLeod et al. 2001). This interpretation is consistent with repeated observations of decreased MPFC activation, relative to baseline, during volitional IHG (Wong, Masse et al. 2007, Goswami, Frances et al. 2011, Norton, Luchyshyn et al. 2013). Despite high inter-individual variability, the MPFC factored importantly into the subtraction analyses when correlated with the task alone (Figure 2.3) illustrating that Control subjects had 
more deactivation (or less activation) than CAD in response to the exercise task. To investigate this outcome further, a secondary analysis of individual brain activation patterns was pursued. This analysis indicated that 16/23 Control and 12/17 CAD patients exhibited a reduction in MPFC activation relative to baseline during the IHG period. However, the number of participants who demonstrated a reduction in MPFC activation and produced a HR response of $\geq 3 \mathrm{bpm}$ to IHG was 9/23 (39\%) in Control and 3/17 $(18 \%)$ in CAD. Thus, the inter-individual variability minimized group-level statistical power in both the specified MPFC regional activation and in the HR response to IHG and, consequently, reflects the reduced brain activation evident in the subtraction analysis in Control subjects.

The variability in brain deactivation patterns outlined above seems to exert a dominant impact on the current results. The high cortical variability in these groups is clear in Figure 2.7, which illustrates that both groups had a lack of consistent MPFC deactivation in response to the IHG task, with some subjects having activation in expected autonomic regions and many individuals having no response at all. In earlier studies from our laboratory, and reports from several other laboratories (Critchley, Corfield et al. 2000, Gianaros, Van Der Veen et al. 2004, Norton, Luchyshyn et al. 2013), HR variations in young, healthy subjects most strongly correlate inversely with deactivation within the HC and MPFC. Moreover, HR-associated effective connectivity exists between the MPFC and $\mathrm{HC}$ in healthy individuals promoting a much larger HR response when both regions are deactivated in concert rather than each region alone (Norton, Luchyshyn et al. 2013). However, HR responses in the current study were much smaller suggesting an effective consequence of cortical "de-coupling". For example, a small HR response was observed in some individuals who did not express deactivation within the MPFC or HC. This pattern was dominated by Control subjects (9/12). To our knowledge, no data exist to address this critical component of changes in cardiovascular control that emerge in aging and diseased individuals.

\subsection{Limitations}

All CAD patients were on a combination of drug therapies including cholesterol lowering statins, beta-blockade, angiotensin-converting enzyme (ACE) inhibitors/angiotensin II 
receptor blockers, calcium channel blockers, diuretics, and anti-platelets including aspirin. ACE-inhibitors and angiotensin II receptor blockers affect vasomotor control but exert minimal effect on cardiac function and should not have impacted the current results. Beta-blockers can reduce baseline HR and interfere with sympathetically-driven changes in HR. However, these effects are largely seen at maximal workloads, which is supported by our data (stress test data, Table 2.1) and are not expected to affect HR responses to the 40\% IHG task where vagal control dominates below 100bpm (Rowell and O'Leary 1990). Furthermore, the heart rate response to the $40 \%$ IHG was not different between groups suggesting that beta-blockade did not influence HR responses at the level of the heart. Finally, as outlined above, CAD patients were capable of mounting a significant HR response during the cardiorespiratory fitness test indicating that the heart's ability to respond to volitional effortful tasks was not altered by the medication.

\subsection{Conclusion}

Overall, the current results indicate that relative to similarly-aged, and apparently healthy individuals, vascular disease impairs functional outcomes in the brain in response to moderate intensity IHG. In particular, the enhanced variability of cortical responses and diminished total cortical activation patterns in CAD are consistent with an overall lower $\mathrm{HR}$ response, promoting the hypothesis that $\mathrm{CAD}$ patients appear to exhibit dysregulation of the brain-heart connection. 


\subsection{References}

ACSM (1995). Guidelines for Exercise Testing and Prescription. L. W. a. Wilkins. Philadelphia: 160.

Anazodo, U. C., J. K. Shoemaker, N. Suskin and K. S. St Lawrence (2013). "An investigation of changes in regional gray matter volume in cardiovascular disease patients, pre and post cardiovascular rehabilitation." Neuroimage. Clin 3: 388-395.

Barberi, E. A., J. S. Gati, B. K. Rutt and R. S. Menon (2000). "A transmit-only/receiveonly (TORO) RF system for high-field MRI/MRS applications." $\underline{\text { Magn Reson. Med }}$ 43(2): 284-289.

Barekatain, M., H. Askarpour, F. Zahedian, M. Walterfang, D. Velakoulis, M. R. Maracy and M. H. Jazi (2014). "The relationship between regional brain volumes and the extent of coronary artery disease in mild cognitive impairment." J. Res. Med. Sci 19(8): 739745.

Basnayake, S. D., A. L. Green and D. J. Paterson (2012). "Mapping the central neurocircuitry that integrates the cardiovascular response to exercise in humans." Exp. Physiol 97(1): 29-38.

Borg, G. (1982). "Ratings of perceived exertion and heart rates during short-term cycle exercise and their use in a new cycling strength test." Int. J. Sports Med 3(3): 153-158.

Burns, S. M. and J. M. Wyss (1985). "The involvement of the anterior cingulate cortex in blood pressure control." Brain Res 340(1): 71-77.

Cabeza, R., S. M. Daselaar, F. Dolcos, S. E. Prince, M. Budde and L. Nyberg (2004). "Task-independent and task-specific age effects on brain activity during working memory, visual attention and episodic retrieval." Cereb. Cortex 14(4): 364-375.

Cechetto, D. F. (2014). "Cortical control of the autonomic nervous system." Exp. Physiol 99(2): 326-331. 
Cechetto, D. F. and S. J. Chen (1990). "Subcortical sites mediating sympathetic responses from insular cortex in rats." Am. J. Physiol 258(1 Pt 2): R245-R255.

Cechetto, D. F. and C. B. Saper (1990). Role of the cerebral cortex in autonomic function, Oxford University Press, New York.

Cheung, R. T. and V. Hachinski (2000). "The insula and cerebrogenic sudden death." Arch. Neurol 57(12): 1685-1688.

Critchley, H. D., D. R. Corfield, M. P. Chandler, C. J. Mathias and R. J. Dolan (2000). "Cerebral correlates of autonomic cardiovascular arousal: a functional neuroimaging investigation in humans." J. Physiol 523 Pt 1: 259-270.

Curtis, B. M. and J. H. O'Keefe, Jr. (2002). "Autonomic tone as a cardiovascular risk factor: the dangers of chronic fight or flight." Mayo Clin. Proc 77(1): 45-54.

Eckberg, D. L., M. Drabinsky and E. Braunwald (1971). "Defective cardiac parasympathetic control in patients with heart disease." N. Engl. J. Med 285(16): 877883.

Fagraeus, L. and D. Linnarsson (1976). "Autonomic origin of heart rate fluctuations at the onset of muscular exercise." J. Appl. Physiol 40(5): 679-682.

Fisk, G. D. and J. M. Wyss (1997). "Pressor and depressor sites are intermingled in the cingulate cortex of the rat." Brain Res 754(1-2): 204-212.

Fletcher, G. F., G. J. Balady, E. A. Amsterdam, B. Chaitman, R. Eckel, J. Fleg, V. F. Froelicher, A. S. Leon, I. L. Pina, R. Rodney, D. A. Simons-Morton, M. A. Williams and T. Bazzarre (2001). "Exercise standards for testing and training: a statement for healthcare professionals from the American Heart Association." Circulation 104(14): 1694-1740.

Ford, G. A. (1999). "Ageing and the baroreflex." Age Ageing 28(4): 337-338. 
Friston, K. J., A. P. Holmes, J. B. Poline, P. J. Grasby, S. C. Williams, R. S. Frackowiak and R. Turner (1995). "Analysis of fMRI time-series revisited." Neuroimage 2(1): 45-53.

Gianaros, P. J., F. M. Van Der Veen and J. R. Jennings (2004). "Regional cerebral blood flow correlates with heart period and high-frequency heart period variability during working-memory tasks: Implications for the cortical and subcortical regulation of cardiac autonomic activity." Psychophysiology 41(4): 521-530.

Goebel, R., F. Esposito and E. Formisano (2006). "Analysis of functional image analysis contest (FIAC) data with brainvoyager QX: From single-subject to cortically aligned group general linear model analysis and self-organizing group independent component analysis." Hum. Brain Mapp 27(5): 392-401.

Goswami, R., M. F. Frances and J. K. Shoemaker (2011). "Representation of somatosensory inputs within the cortical autonomic network." Neuroimage 54(2): 12111220.

Goswami, R., M. F. Frances, C. D. Steinback and J. K. Shoemaker (2012). "Forebrain organization representing baroreceptor gating of somatosensory afferents within the cortical autonomic network." J. Neurophysiol 108(2): 453-466.

Gribbin, B., T. G. Pickering and P. Sleight (1969). "Decrease in baroreflex sensitivity with increasing arterial pressure and with increasing age." Br. Heart J 31(6): 791-798.

Hagler, D. J., Jr., A. P. Saygin and M. I. Sereno (2006). "Smoothing and cluster thresholding for cortical surface-based group analysis of fMRI data." Neuroimage 33(4): 1093-1103.

Hollander, A. P. and L. N. Bouman (1975). "Cardiac acceleration in man elicited by a muscle-heart reflex." J. Appl. Physiol 38(2): 272-278.

Kimmerly, D. S., D. D. O'Leary, R. S. Menon, J. S. Gati and J. K. Shoemaker (2005). "Cortical regions associated with autonomic cardiovascular regulation during lower body negative pressure in humans." J. Physiol 569(Pt 1): 331-345. 
Macey, P. M., P. Wu, R. Kumar, J. A. Ogren, H. L. Richardson, M. A. Woo and R. M. Harper (2012). "Differential responses of the insular cortex gyri to autonomic challenges." Auton. Neurosci 168(1-2): 72-81.

Mancia, G., J. Cleroux, A. Daffonchio, A. U. Ferrari, C. Giannattasio and G. Grassi (1991). "Reflex control of circulation in the elderly." Cardiovasc. Drugs Ther 4 Suppl 6: 1223-1228.

Mancia, G., J. Iannos, G. G. Jamieson, R. H. Lawrence, P. R. Sharman and J. Ludbrook (1978). "Effect of isometric hand-grip exercise on the carotid sinus baroreceptor reflex in man." Clin. Sci. Mol. Med 54(1): 33-37.

Mark, A. L., R. G. Victor, C. Nerhed and B. G. Wallin (1985). "Microneurographic studies of the mechanisms of sympathetic nerve responses to static exercise in humans." Circ Res 57(3): 461-469.

Martins, I. J., E. Hone, J. K. Foster, S. I. Sunram-Lea, A. Gnjec, S. J. Fuller, D. Nolan, S. E. Gandy and R. N. Martins (2006). "Apolipoprotein E, cholesterol metabolism, diabetes, and the convergence of risk factors for Alzheimer's disease and cardiovascular disease." Mol. Psychiatry 11(8): 721-736.

Matthews, S. C., M. P. Paulus, A. N. Simmons, R. A. Nelesen and J. E. Dimsdale (2004). "Functional subdivisions within anterior cingulate cortex and their relationship to autonomic nervous system function." Neuroimage 22(3): 1151-1156.

Mitchell, J. H., D. R. Reeves, Jr., H. B. Rogers, N. H. Secher and R. G. Victor (1989). "Autonomic blockade and cardiovascular responses to static exercise in partially curarized man." J. Physiol 413: 433-445.

Monahan, K. D., F. A. Dinenno, D. R. Seals, C. M. Clevenger, C. A. DeSouza and H. Tanaka (2001). "Age-associated changes in cardiovagal baroreflex sensitivity are related to central arterial compliance." Am. J. Physiol Heart Circ. Physiol 281(1): H284-H289. 
Norton, K. N., T. A. Luchyshyn and S. J. Kevin (2013). "Evidence for a medial prefrontal cortex-hippocampal axis associated with heart rate control in conscious humans." Brain Res 1538: 104-115.

Oppenheimer, S. M., A. Gelb, J. P. Girvin and V. C. Hachinski (1992). "Cardiovascular effects of human insular cortex stimulation." Neurology 42(9): 1727-1732.

Owens, N. C. and A. J. Verberne (2001). "Regional haemodynamic responses to activation of the medial prefrontal cortex depressor region." Brain Res 919(2): 221-231.

Raichle, M. E., A. M. MacLeod, A. Z. Snyder, W. J. Powers, D. A. Gusnard and G. L. Shulman (2001). "A default mode of brain function." Proc. Natl. Acad. Sci. U. S. A 98(2): 676-682.

Resstel, L. B., K. B. Fernandes and F. M. Correa (2004). "Medial prefrontal cortex modulation of the baroreflex parasympathetic component in the rat." Brain Res $\mathbf{1 0 1 5}(1-$ 2): 136-144.

Reuter-Lorenz, P. (2002). "New visions of the aging mind and brain." Trends Cogn Sci 6(9): 394.

Reuter-Lorenz, P. A. and D. C. Park (2010). "Human neuroscience and the aging mind: a new look at old problems." J. Gerontol. B Psychol. Sci. Soc. Sci 65(4): 405-415.

Roberts, R. O., D. S. Knopman, Y. E. Geda, R. H. Cha, V. L. Roger and R. C. Petersen (2010). "Coronary heart disease is associated with non-amnestic mild cognitive impairment." Neurobiol. Aging 31(11): 1894-1902.

Rowell, L. B. and D. S. O'Leary (1990). "Reflex control of the circulation during exercise: chemoreflexes and mechanoreflexes." J Appl Physiol (1985) 69(2): 407-418.

Ruggiero, D. A., S. Mraovitch, A. R. Granata, M. Anwar and D. J. Reis (1987). "A role of insular cortex in cardiovascular function." J. Comp Neurol 257(2): 189-207. 
Seals, D. R., J. A. Taylor, A. V. Ng and M. D. Esler (1994). "Exercise and aging: autonomic control of the circulation." Med. Sci. Sports Exerc 26(5): 568-576.

Shoemaker, J. K., K. N. Norton, J. Baker and T. Luchyshyn (2014). "Topic Series: Autonomic responses to exercise: Forebrain organization for autonomic cardiovascular control." Auton. Neurosci.

Talairach, J. and P. Tournoux (1988). Co-Planar Stereotaxic Atlas of the Human Brain. New York, NY, Thieme Medical Publishers Inc.

Thayer, J. F., J. J. Sollers, III, D. M. Labiner, M. Weinand, A. M. Herring, R. D. Lane and G. L. Ahern (2009). "Age-related differences in prefrontal control of heart rate in humans: a pharmacological blockade study." Int. J. Psychophysiol 72(1): 81-88.

Verberne, A. J. (1996). "Medullary sympathoexcitatory neurons are inhibited by activation of the medial prefrontal cortex in the rat." Am. J. Physiol 270(4 Pt 2): R713R719.

Verberne, A. J. and N. C. Owens (1998). "Cortical Modulation of the Cardiovascular System." Progress in Neurobiology 54(2): 149-168.

Williamson, J. W. (2010). "The relevance of central command for the neural cardiovascular control of exercise." Exp. Physiol 95(11): 1043-1048.

Williamson, J. W., R. McColl and D. Mathews (2003). "Evidence for central command activation of the human insular cortex during exercise." J. Appl. Physiol (1985. ) 94(5): 1726-1734.

Wong, S. W., N. Masse, D. S. Kimmerly, R. S. Menon and J. K. Shoemaker (2007). "Ventral medial prefrontal cortex and cardiovagal control in conscious humans." Neuroimage 35(2): 698-708.

Yasui, Y., C. D. Breder, C. B. Saper and D. F. Cechetto (1991). "Autonomic responses and efferent pathways from the insular cortex in the rat." J. Comp Neurol 303(3): 355374. 
Zulli, R., F. Nicosia, B. Borroni, C. Agosti, P. Prometti, P. Donati, V. M. De, D. Turini, G. Romanelli, V. Grassi and A. Padovani (2008). "Increased prevalence of silent myocardial ischaemia and severe ventricular arrhythmias in untreated patients with Alzheimer's disease and mild cognitive impairment without overt coronary artery disease." Clin. Neurol. Neurosurg 110(8): 791-796. 


\section{Chapter 3}

\section{Impact of Long-Term Endurance Training Versus Guideline-Based Physical Activity on Brain Structure in Healthy Aging}

(Published in Front Aging Neurosci. 2016 Jun 30;8:155. doi: 10.3389/fnagi.2016.00155. eCollection 2016)

\subsection{Introduction}

Atrophy of cortical gray matter is a hallmark of aging, with tissue loss beginning early in the third decade of life (Good, Johnsrude et al. 2001, Raz, Lindenberger et al. 2005). Importantly, strong inverse relationships exist between cortical thickness and age-related dementias (Lerch, Pruessner et al. 2005, Du, Schuff et al. 2007). Furthermore, parallel declines also occur between cognitive function and structural changes in the medial prefrontal cortex, dorso-lateral prefrontal cortex, hippocampus, anterior cingulate, amygdala, and insula (Coffey, Wilkinson et al. 1992, Raz, Gunning et al. 1997, Good, Johnsrude et al. 2001, Raz, Lindenberger et al. 2005). Given the economic, social, and personal burden associated with age-related neural deterioration, identifying strategic mechanisms to prevent declines in structural and functional brain health before they begin represents an imperative public health goal.

Exercise has emerged as a potent stimulus for cognitive improvement in older adults who are sedentary and experience cognitive decline (Kramer, Colcombe et al. 2005, Voss, Nagamatsu et al. 2011, Chaddock, Erickson et al. 2012). However, the extent to which exercise training can modify cortical thickness or subcortical gray matter volume remains relatively unstudied, with considerable variability across studies. For example, Erickson et al. observed rather rapid increases in $\mathrm{HC}$ volume in just 6 weeks of a walking intervention performed by sedentary, elderly individuals (Erickson, Voss et al. 2011). This study shows that exercise is neuroprotective when applied in a late-onset model after atrophy likely has begun, as suggested by cognitive impairment. In a whole brain analysis, Rovio et al. reported that people who were physically active during middle age 
( $\sim 50$ years of age) expressed greater frontal lobe cortical thickness at a 21-year follow-up assessment, compared to an inactive control group (Rovio, Spulber et al. 2010). Similarly, Erikson et al. showed a threshold effect of walking distance over a 9-year follow-up period on cortical thickness in the prefrontal, temporal and hippocampus regions (Erickson, Raji et al. 2010). These studies show that activity during the middle age period may exert neuroprotective benefits to a limited portion of the brain later in life. This idea stands in contrast to the knowledge that overall risk of disease and mortality relates to cardiorespiratory fitness (Defina, Willis et al. 2013, Barry, Baruth et al. 2014) or daily energy expenditure (Booth, Roberts et al. 2012) suggesting that higher gains may be possible than those achieved with mild to moderate interventions such as those provided through published guidelines for active living (ACSM 1995). Also, the research linking individual differences in physical activity, and training-related changes in fitness to brain health, has led to a hypothesis that cardiorespiratory fitness is a critical mediator of these benefits (Etnier, Nowell et al. 2006, Angevaren, Aufdemkampe et al. 2008, Smith 2012). If so, then a dose-response pattern should exist in the building of cortical mass reserve through the life-long pursuit of high fitness levels.

Master's Athletes (MA) provide a unique model to assess the extent to which long-term training can affect brain metrics. Compared to a cohort of inactive elderly controls, Tseng et al. observed greater posterior cortical thickness in the cuneus and precuneus in a small group of older (72 years) MA (Tseng, Uh et al. 2013). These results are interesting when considering previous evidence for greater sensitivity of anterior brain regions for agerelated brain atrophy (Raz, Gunning et al. 1997, Good, Johnsrude et al. 2001, Raz, Lindenberger et al. 2005). The relative lack of exercise effect in these MA in frontal brain regions is of importance because it suggests either a transient effect of exercise on frontal brain mass, or a minimized effect of high training loads on neuroplastic outcomes. Variations in the above studies may also reflect issues such as small sample size, focus on narrow age ranges, varying baseline conditions, short versus long-term training periods, timing of the exercise onset, and/or emphasis on univariate group-based or region-ofinterest analysis. In addition, the use of sedentary older individuals as the control condition in many studies introduces a higher probability of undetected nuisance variables such as latent cerebrovascular damage, endothelial dysfunction, and 
subthreshold neurological impairments. These possibilities raise questions regarding the maximal exercise-induced benefit possible across the adult age span, as well as the appropriate control group for such studies. In addition, it may be that adhering to accepted guidelines for active living (ACSM 1995) achieves benefits that are not enhanced by additional training loads.

This study attempts to expose the maximal benefit to cortical and subcortical gray matter possible in the context of exercise training. To avoid concerns about subthreshold agerelated nuisance variables related to physical inactivity, and to directly relate the differences between guideline-based training versus high training loads, world-class MA as well as regularly active, but non-competitive, healthy volunteers were studied. We tested the overall hypothesis that gray matter adaptations to exercise follow a dosedependent pattern in each of the cortical and subcortical gray matter regions.

\subsection{Methods}

\subsubsection{Participants}

A total of 32 individuals participated in this study. Observations were made in 16 elitelevel middle-aged and older Masters Athletes (MA) who trained and raced in the sport of triathlon at the professional or national level for $>30$ years $(53 \pm 6$ years of age (4 female), training $>15 \mathrm{~h} / \mathrm{wk} ; \mathrm{VO}_{2 \max }=55 \pm 10 \mathrm{ml} / \mathrm{kg} / \mathrm{min}$ ), and 16 similarly-aged healthy, active (HA) control subjects who met the age-appropriate health recommendations for exercise for at least 5 years $\left(58 \pm 9\right.$ years, $\left(6\right.$ female); $\mathrm{VO}_{2 \max }=38 \pm 7 \mathrm{ml} / \mathrm{kg} / \mathrm{min}$ ). Table 3.1 provides group characteristics. All subjects were right-handed, non-smokers, free of medications, and did not have diagnosed hypertension, diabetes, vascular or neurological/psychological impairments. Testing of menstruating females occurred during days 1-14 of the menstrual cycle, with day one representing the first day of menstruation. The post-menopausal women were not taking hormone replacement therapy. The University of Western Ontario Health Sciences Ethics Review Board approved this study and each participant provided informed, written consent. The study adhered to the Declaration of Helsinki. 


\subsubsection{Assessment of Cardiorespiratory Fitness}

A graded treadmill exercise test, conducted under standard clinical observation, provided information regarding each subject's peak oxygen uptake $\left(\mathrm{VO}_{2 \mathrm{max}}\right)$. During this test, analysis of expired air samples occurred over 3-second intervals until the point of volitional exhaustion. Based on the American College of Sports Medicine guidelines (ACSM 1995), $\mathrm{VO}_{2 \max }$ was determined by meeting at least three of the following criteria: (1) $\mathrm{VO}_{2}$ ceased to increase with increasing workloads (plateau); (2) heart rate reached the age-predicted value (220-age); (3) respiratory exchange ratio > 1.0; and (4) blood lactate $>8.0 \mathrm{mmol} / \mathrm{L}$. These methods have been tested and validated extensively in previous studies of older subjects (Levine 2008, Fujimoto, Prasad et al. 2010).

\subsubsection{Physiological Data Acquisition}

Participants completed two separate experimental sessions: 1) physiological laboratory recording, and 2) magnetic resonance neuroimaging session (MRI; Robarts Research Institute Centre for Functional and Metabolic Imaging). The two sessions were performed at the same time of day and separated by a minimum period of 1 week. Participants practiced the experimental procedures prior to their first test session. Participants reported to the laboratory following a $12 \mathrm{~h}$ abstinence from nicotine, alcohol, caffeine and intense physical exertion. Venous blood sampling occurred following 30mins of quiet, supine rest. Analysis of these samples assessed and confirmed baseline levels of blood-borne acute phase inflammatory markers (hsCRP) and glycemic status. Heart rate was measured using a standard three-lead electrocardiogram. Finger photoplethysmography (Finometer; Finapres Medical Systems, Amsterdam, The Netherlands) provided continuous measures of arterial blood pressure following calibration to manual sphygmomanometer blood pressure measurements obtained throughout the protocol. Data were collected using LabChart7 and PowerLab data acquisition system (ADInstruments). Neurological screening was also completed during the laboratory session to exclude dementia (The Montreal Cognitive Assessment) and confirm intact executive function (Trail making tests $\mathrm{A}$ and $\mathrm{B})$. 


\subsubsection{Neuroimaging Data Acquisition}

Participants completed a structural MRI session following a $12 \mathrm{~h}$ abstinence from nicotine, alcohol, caffeine and intense physical exertion. All imaging data were collected using a whole body 3-Tesla imaging system (Magnetom Prisma, Siemens Medical Solutions, Erlangen, Germany) with a 32-channel head coil (Barberi, Gati et al. 2000). A high-resolution T1-weighted structural volume was acquired with a 3D MPRAGE sequence at the beginning of the scanning session (sagittal, matrix 256x240mm, voxel resolution 1.0x1.0x1.0mm, 1mm slice thickness, no gap, flip angle $9^{\circ}$, TE $=2.98 \mathrm{~ms}$, TI $=900 \mathrm{~ms}$, TR $=2.3 \mathrm{~ms}$ ). Head movement was limited during the experimental session within a head cradle packed with foam padding, and each subject received instruction to avoid head movements during the scanning period.

\subsubsection{Neuroimaging Data Analysis}

We used the analysis approaches best suited to measure gray and white matter cortically and subcortically, from high-resolution MRI images.

\subsubsection{Subcortical Level}

Semiautomatic software quantified ventricular and subcortical volumes, as well as total intracranial volume, total gray matter and white matter hypointensities (Freesurfer Image Analysis Suite, http://surfer.nmr.mgh.harvard.edu/). The technical details of these procedures are described in prior publications (Dale and Sereno 1993, Dale, Fischl et al. 1999, Fischl and Dale 2000, Fischl, Liu et al. 2001, Fischl, Salat et al. 2002, Fischl, Salat et al. 2004). Briefly, this processing included motion correction and averaging (Reuter, Schmansky et al. 2012) of T1-weighted images, removal of non-brain tissue (Segonne, Dale et al. 2004), automated Talairach transformation, segmentation of the subcortical white matter and deep gray matter volumetric structures of interest (including hippocampus, amygdala, anterior cingulate cortex, lateral ventricles) (Fischl, Salat et al. 2002, Fischl, Salat et al. 2004), intensity normalization (Sled, Zijdenbos et al. 1998), delineation of the gray and white matter boundary, automated topology correction (Fischl, Salat et al. 2004, Segonne, Dale et al. 2004) and surface deformation following intensity gradients for optimal placement of gray/white and gray/cerebrospinal fluid 
borders at the location where the greatest shift in intensity defines the transition to the other tissue (Dale and Sereno 1993, Dale, Fischl et al. 1999, Fischl and Dale 2000). Measurement of ventricular volume is particularly amenable to segmentation due to the high signal intensity contrast between cerebrospinal fluid and surrounding brain tissue in $\mathrm{T}_{1}$-weighted MRI. Of note, ventricle volume segmentation included only the lateral ventricles. Adjusting regional volumes to intracranial volume accounted for the potential impact of sex and height on structural outcomes. Subcortical results were corrected for multiple comparisons at FDR, $\mathrm{p}<0.05$.

Cognitive and age-related cortical decline appear to overlap in cortical sites such as the MPFC and IC; as well at specific subcortical sites including the HC, ACC, and amygdala (Coffey, Wilkinson et al. 1992, Raz, Gunning et al. 1997, Good, Johnsrude et al. 2001, Raz, Lindenberger et al. 2005). Thus, we used a region-of-interest (ROI) analysis to focus on these most vulnerable deep gray matter sites (HC, amygdala and ACC) as well as the fluid-filled lateral ventricles (Good, Johnsrude et al. 2001, Resnick, Pham et al. 2003, Raz, Lindenberger et al. 2005, Kennedy, Erickson et al. 2009, Raz, Ghisletta et al. 2010). White-matter hypointensities provided an index of cerebrovascular disease and cognitive decline (Debette and Markus 2010). In this case, Freesurfer provides reliable sensitivity in measuring white matter damage in non-demented older adults (Leritz, Shepel et al. 2014).

\subsubsection{Cortical Level}

Cortical thickness analysis was performed using Brain Voyager 2.8.4 (BVQX, Brain Innovation, Maastricht, Netherlands). The Lapalace method provided measures of cortical thickness for each subject's right and left hemisphere (Jones, Buchbinder et al. 2000). An inter-subject cortical alignment procedure reduced the effect of anatomical variability and improved the spatial correspondence of cortical areas between individual brains. Spatial intensity inhomogeneities in the original $T_{1}$-weighted scans were corrected, converted into volumes with 1-mm isotropic voxel resolution using sincinterpolation, and transformed spatially into standard Talairach space. Once normalized, an automatic segmentation of white-matter and gray-matter boundaries was applied, and images were resampled to $0.5 \mathrm{~mm}$ isovoxel resolution. Manual correction removed 
topological errors such as "bridges" and remaining fragments of dura mater or cerebellum on a slice-by-slice and individual basis. The reconstructed cortical hemispheres were morphed into a folded three-dimensional mesh. Cortex-based-alignment followed, producing a spherical representation of the folded cortex and finally for each hemisphere. The curvature information of individual brains enabled cortex-based inter-subject alignment (Goebel, Esposito et al. 2006), and resulted in average curvature maps for each hemisphere, for each group. Each mesh resulted from averaging 16 individual datasets (16 MA and 16 HA).

\subsubsection{Statistical Analysis}

A two-tailed unpaired t-test assessed group-level hemodynamic and anthropometric characteristics, as well as the effect of group on cortical thickness ( $<<0.05$; Systat Software 12.5, 2011). Data are presented as mean \pm standard deviation. Overlap existed in the fitness levels of the MA and HA group members. Therefore, following a combining of groups, multiple linear regression analysis assessed the independence of the relationship between subcortical volumes and cortical thickness after adjusting for age, $\mathrm{VO}_{2 \max }$, body mass index (BMI), and left cardiac ventricular mass (indexed to body surface area), as they exert strong effects on the morphology of the cortex (Table 3.2) (Barnes, Yaffe et al. 2003). A threshold for significance was set at $p<0.05$. All regression analyses passed the normality test, as assessed by the Shapiro-Wilk test for normal distribution. The effect of high cardiorespiratory fitness on the age-related decay of cortical and subcortical mass at the specified regions of interest was determined using linear regression and was compared using Student's $t$-test.

To assess the effects of fitness and age on regional brain tissue health, we performed a full cortex correlation analysis in Brain Voyager using each individual's $\mathrm{VO}_{2 \max }$ and age in years. The final correlation maps were adapted by switching off the positive or negative correlation values respectively, increasing the minimum threshold value $(\mathrm{p}<0.005)$, and adding a cluster threshold $\left(10 \mathrm{~mm}^{2}\right)$. 


\subsection{Results}

Table 3.1 provides descriptive group information. Participant ages ranged from 45 to 73 years in HA and 45 to 67 years in MA, with a mean age of 58 years and 53 years, respectively. Overall, the sample was $31 \%$ female. Groups did not differ with respect to age, sex, years of education, blood pressure, heart rate and/or BMI. The HA group demonstrated near $100 \%$ of age-predicted $\mathrm{VO}_{2 \max }$, consistent with self-report confirmation of adherence to age-appropriate physical activity guidelines (ACSM 1995). Compared to HA participants, MA had a greater $\mathrm{VO}_{2 \max }$, and greater left cardiac ventricular mass. The MoCA and Trail Making Test B scores were not different between groups. HA participants expressed a slightly longer time to completion in Trail Making Test A. Plasma glucose, circulating triglycerides, and systolic blood pressures were greater in HA than MA, but all values were within healthy ranges. Circulating hsCRP and lipid levels, as well as total brain volume indicators, were similar between groups.

\subsubsection{Subcortical Gray Matter}

When indexed to total intracranial volume, average subcortical gray matter volumes were not different between HA and MA (Table 3.2).

The mean rate of ventricular volume enlargement was $0.03 \%$ per year in HA and $0.04 \%$ per year in MA (P=0.75; Figure 3.1). The mean annual rate of atrophy at the ACC was $0.0006 \%$ in HA and $-0.0002 \%$ in MA, which was not different between groups $(\mathrm{p}=0.31)$. The mean rate of atrophy at the right $\mathrm{HC}$ (HA: -0.0009\%; MA: -0.0006\%) was not different between groups $(\mathrm{p}=0.57)$, nor was it different at the left $\mathrm{HC}(\mathrm{p}=0.25)$ in either HA $(-0.0016 \%)$ or MA $(0.0002 \%)$. The right amygdala also showed no difference in rate of volume change $(\mathrm{p}=0.44)$ in either HA $(-0.0007 \%)$ or MA $(-0.0003 \%)$, which was consistent with the left amygdala ( $\mathrm{p}=0.11$; HA: $-0.0006 \%$; MA: 0.0002\%). Pearson Correlation analysis confirmed linear dependence between age and ventricle volume $(\mathrm{r}=$ $0.49, \mathrm{p}<0.001)$, and an inverse correlation between age and $\mathrm{VO}_{2 \max }(\mathrm{r}=-0.71, \mathrm{p}<$ $0.001)$. 


\subsubsection{Region of Interest Analysis}

Average volumes at the lateral ventricles, ACC, the right and left $\mathrm{HC}$, and the right amygdala were similar in the MA and HA groups (Table 3.2). However, the left amygdala was larger in MA versus HA $(\mathrm{p}<0.05)$.

\subsubsection{Covariate Analysis}

Pearson correlation analysis revealed that ventricle volume correlated inversely, albeit weakly, with $\mathrm{VO}_{2 \max }(\mathrm{r}=-0.28, \mathrm{p}=0.05)$. Multiple linear regression revealed age as the strongest predictor of lateral ventricle volume $(\mathrm{p}<0.001)$, right $\mathrm{HC}(\mathrm{p}<0.05)$, right amygdala $(\mathrm{p}<0.05)$, and ACC volumes $(\mathrm{p}<0.05$, Table 3.3).

\subsubsection{Cortical Gray Matter}

Total gray matter was greater in MA than in HA ( $\mathrm{p}<0.05)$. An unpaired t-test revealed group differences in cortical thickness throughout a wide range of cortical areas ( $\mathrm{p}<0.005$, corrected for multiple comparisons by cluster threshold $=10$ voxels; Figure 3.2, Tables 3.4-3.5). The most prominent differences $(>1.5 \mathrm{~mm})$ were observed in occipital and temporal regions. Smaller clusters of group differences in cortical thickness $(>1 \mathrm{~mm})$ were also detected within the frontal lobe and medial plane. Additionally, there was a dominant lateralization pattern to the left hemisphere, which had greater cortical thickness in many regions, than the right hemisphere.

The annual rate of cortical atrophy across the age span (Figure 3.3) was not different between HA and MA at the right IC (HA: -0.53\%; MA: -0.69\%; p = 0.82), left IC (HA: $0.52 \%$; MA: $-1.79 \% ; \mathrm{p}=0.32$ ), the right MPFC (HA: $-1.23 \%$; MA: $-1.37 \%$; $=0.96$ ), or the left MPFC (HA: 0.044\%; MA: 0.29\%; $\mathrm{p}=0.88$ ). Also, the mean annual rate of change did not differ between groups at the left precentral gyrus (HA: -0.01\%; MA: $0.61 \% ; \mathrm{p}=0.33)$, the right postcentral gyrus (HA: $-0.17 \%$; MA: $0.11 \% ; \mathrm{p}=0.95)$ and the left postcentral gyrus (HA: $-0.009 \%$; MA: $0.82 \%$; $\mathrm{p}=0.11$ ). The effect of age on cortical thickness at the right precentral gyrus was different between groups $(\mathrm{p}<0.05)$ where little change was observed in HA $(-0.28 \%)$ but a progressive increase in cortical thickness was observed in MA $(0.12 \%)$. 
In a whole-brain, voxel-wise negative correlation analysis, age correlated negatively with cortical thickness in the parietal, temporal and frontal lobes (Figure 3.4). Further, a negative correlation analysis revealed regions of cortical thickness that correlated negatively (i.e., was thinner) with $\mathrm{VO}_{2 \max }$ (Figure 3.5) in MA versus HA; these regions were few in number and were concentrated around the occipital and parietal cortices.

\subsubsection{Region of Interest Analysis}

Cortical thickness was greater in MA than HA at each region of interest including the bilateral IC, MPFC, precentral and postcentral gyri (Figure 3.6).

\subsubsection{Covariate Analysis}

As depicted in Figure 3.7, cortical thickness and $\mathrm{VO}_{2 \max }$ exhibited strong correlations across much of the cerebral cortex. Specific results from the multiple linear regression indicated that across all regions of interest, $\mathrm{VO}_{2 \max }$ was the strongest predictor of cortical thickness (Table 3.6) with the exception of the right precentral gyrus and left postcentral gyrus. 
Table 3.1 Anthropometric and baseline hemodynamic data (mean \pm SD).

\begin{tabular}{|c|c|c|}
\hline & Healthy Active & Masters Athletes \\
\hline Age (vears) & $58 \pm 9$ & $53 \pm 6$ \\
\hline & Range: $45-73$ & Range: $45-67$ \\
\hline Resting Blood Pressure $(\mathrm{mmHg})$ & $118 / 73 \pm 9$ & $118 / 78 \pm 9$ \\
\hline Resting Heart Rate (bpm) & $58 \pm 8$ & $55 \pm 9$ \\
\hline BMI $\left(\mathrm{kg} / \mathrm{m}^{2}\right)$ & $26 \pm 4$ & $23 \pm 3$ \\
\hline & $38 \pm 7$ & $55 \pm 10^{*}$ \\
\hline $\mathrm{V}_{2 \max }(\mathrm{mL} / \mathrm{Kg} / \mathrm{min})$ & Range: $26-51$ & Range: $33-67$ \\
\hline Age-predicted $\mathrm{VO}_{2 \max }(\%)$ & $94 \pm 19$ & $125 \pm 26^{*}$ \\
\hline LVM $(\mathrm{g})$ & $64 \pm 14$ & $77 \pm 17 *$ \\
\hline Education (years) & $12 \pm 2$ & $10 \pm 3$ \\
\hline Trail Making Score A (secs) & $31 \pm 8$ & $23 \pm 10 *$ \\
\hline Trail Making Score B (secs) & $59 \pm 21$ & $53 \pm 23$ \\
\hline MoCA Score & $28 \pm 2$ & $27 \pm 2$ \\
\hline Fasting Glucose $(\mathrm{mmol} / \mathrm{L})$ & $5.16 \pm 0.35$ & $4.89 \pm 0.31 *$ \\
\hline hsC-Reactive Protein $(\mathrm{mg} / \mathrm{L})$ & $1.54 \pm 3.22$ & $1.49 \pm 1.17$ \\
\hline Cholesterol $(\mathrm{mmol} / \mathrm{L})$ & $4.46 \pm 0.70$ & $4.23 \pm 0.76$ \\
\hline Triglycerides $(\mathrm{mmol} / \mathrm{L})$ & $0.92 \pm 0.45$ & $0.61 \pm 0.22 *$ \\
\hline High Density Lipoprotein $(\mathrm{mmol} / \mathrm{L})$ & $1.50 \pm 0.40$ & $1.53 \pm 0.35$ \\
\hline Low Density Lipoprotein $(\mathrm{mmol} / \mathrm{L})$ & $2.54 \pm 0.60$ & $2.42 \pm 0.79$ \\
\hline $\mathrm{HbA1c}$ & $0.06 \pm 0.002$ & $0.06 \pm 0.002$ \\
\hline Gray Matter Volume (mL) & $655 \pm 51$ & $691 \pm 55^{*}$ \\
\hline White Matter Hypointensities $\left(\mathrm{mm}^{3}\right)$ & $1924 \pm 1221$ & $1501 \pm 708$ \\
\hline Total Intracranial Volume $\left(\mathrm{mm}^{3}\right)$ & $1.6 \times 10^{6} \pm 1.5 \times 10^{5}$ & $1.6 \times 10^{6} \pm 1.6 \times 10^{5}$ \\
\hline
\end{tabular}

$\mathrm{BMI}$, body mass index; $\mathrm{VO}_{2 \max }$, maximal oxygen consumption; percent of age-predicted $\mathrm{VO}_{2 \max }$ based on ACSM guidelines, 1995; LVM, left ventricular mass at the heart; education in years including high school; MoCA: Montreal Cognitive Assessment (scored out of 30 possible points); hs, high sensitivity. Cardiac output and LVM indexed to body surface area. *different from healthy active, $\mathrm{p}<0.05$. 
Table 3.2 Whole-brain subcortical volumes.

\begin{tabular}{lccccc}
\hline Subcortical Site & $\begin{array}{c}\text { Mean MA } \\
\left(\mathbf{m m}^{\mathbf{3}}\right)\end{array}$ & $\begin{array}{c}\text { Mean HA } \\
\left(\mathbf{m m}^{\mathbf{3}}\right)\end{array}$ & $\begin{array}{c}\text { Difference } \\
(\mathbf{M A - H A})\end{array}$ & P value & $\begin{array}{c}\text { Corrected } \\
\text { P value }\end{array}$ \\
\hline Lateral Ventricles & 0.0119 & 0.0136 & -0.0017 & 0.41 & 0.55 \\
Brain Stem & 0.0301 & 0.0140 & 0.0161 & 0.27 & 0.54 \\
CSF & 0.0477 & 0.0007 & 0.0470 & 0.71 & 0.74 \\
L Cerebellum WM & 0.0097 & 0.0092 & 0.0005 & 0.15 & 0.38 \\
R Cerebellum WM & 0.0276 & 0.0094 & 0.0181 & 0.29 & 0.54 \\
L Cerebellum C & 0.0367 & 0.0320 & 0.0047 & 0.14 & 0.38 \\
R Cerebellum C & 0.0603 & 0.0338 & 0.0265 & 0.47 & 0.58 \\
L Thalamus & 0.0464 & 0.0054 & 0.0411 & 0.63 & 0.68 \\
R Thalamus & 0.0197 & 0.0045 & 0.0152 & 0.24 & 0.54 \\
L Caudate & 0.0236 & 0.0021 & 0.0215 & 0.33 & 0.54 \\
R Caudate & 0.0085 & 0.0022 & 0.0063 & 0.09 & 0.38 \\
L Putamen & 0.0471 & 0.0030 & 0.0441 & 0.11 & 0.38 \\
R Putamen & 0.0210 & 0.0029 & 0.0182 & 0.28 & 0.54 \\
L Pallidum & 0.0273 & 0.0008 & 0.0264 & 0.39 & 0.55 \\
R Pallidum & 0.0078 & 0.0010 & 0.0068 & 0.10 & 0.38 \\
L Hippocampus & 0.0342 & 0.0026 & 0.0316 & 0.48 & 0.58 \\
R Hippocampus & 0.0097 & 0.0020 & 0.0077 & 0.12 & 0.38 \\
L Amygdala & 0.0034 & 0.0009 & 0.0024 & $0.03 *$ & 0.35 \\
R Amygdala & 0.0109 & 0.0010 & 0.0099 & 0.15 & 0.38 \\
L Accumbens & 0.0014 & 0.0002 & 0.0012 & $0.02^{*}$ & 0.35 \\
R Accumbens & 0.0235 & 0.0003 & 0.0232 & 0.35 & 0.54 \\
L Ventral DC & 0.0046 & 0.0023 & 0.0024 & $0.04 *$ & 0.35 \\
R Ventral DC & 0.0053 & 0.0022 & 0.0031 & $0.05 *$ & 0.35 \\
Posterior CC & 0.0256 & 0.0006 & 0.0250 & 0.38 & 0.55 \\
Mid-Posterior CC & 0.0497 & 0.0003 & 0.0494 & 0.74 & 0.74 \\
Central CC & 0.0358 & 0.0003 & 0.0355 & 0.53 & 0.59 \\
Mid-Anterior CC & 0.0225 & 0.0003 & 0.0223 & 0.33 & 0.54 \\
Anterior CC & 0.0347 & 0.0006 & 0.0342 & 0.51 & 0.59 \\
\hline
\end{tabular}

All values indexed to total intracranial volume. L: left; R: right; WM: white matter; GM: gray matter; C: cortex; DC: dorsal column; CC: cingulate cortex. *Different from HA, $\mathrm{p}<0.05$. 
Table 3.3 Results of multiple linear regression of ROI subcortical volumes against anthropometric covariates.

\begin{tabular}{|c|c|c|c|c|c|}
\hline & & Std. Coefficient & Std. Error & P value & Power ( $\alpha$ 0.05) \\
\hline \multirow[t]{4}{*}{ Lateral Ventricles } & Age & 0.000483 & 0.000138 & 0.001 & 0.997 \\
\hline & $\mathrm{VO}_{2 \max }$ & 0.000146 & 0.000117 & 0.218 & - \\
\hline & Body Mass Index & -0.000273 & 0.000277 & 0.329 & - \\
\hline & L Ventricular Mass & -0.000122 & 0.000058 & 0.041 & - \\
\hline \multirow[t]{4}{*}{ L Hippocampus } & Age & -0.000016 & 0.000009 & 0.072 & - \\
\hline & $\mathrm{VO}_{2 \max }$ & -0.000004 & 0.000008 & 0.597 & - \\
\hline & Body Mass Index & 0.000006 & 0.000018 & 0.751 & - \\
\hline & L Ventricular Mass & -0.000002 & 0.000004 & 0.675 & - \\
\hline \multirow[t]{4}{*}{ R Hippocampus } & Age & -0.000015 & 0.000008 & 0.049 & 0.894 \\
\hline & $\mathrm{VO}_{2 \max }$ & 0.000002 & 0.000006 & 0.705 & - \\
\hline & Body Mass Index & 0.000019 & 0.000015 & 0.184 & - \\
\hline & L Ventricular Mass & -0.000005 & 0.000003 & 0.135 & - \\
\hline \multirow{4}{*}{ L Amygdala } & Age & -0.000003 & 0.000003 & 0.373 & - \\
\hline & $\mathrm{VO}_{2 \max }$ & 0.000005 & 0.000002 & 0.073 & - \\
\hline & Body Mass Index & 0.000003 & 0.000006 & 0.606 & - \\
\hline & L Ventricular Mass & -0.000001 & 0.000001 & 0.349 & - \\
\hline \multirow[t]{4}{*}{ R Amygdala } & Age & -0.000008 & 0.000003 & 0.009 & 0.937 \\
\hline & $\mathrm{VO}_{2 \text { max }}$ & -0.000001 & 0.000002 & 0.742 & - \\
\hline & Body Mass Index & 0.000008 & 0.000006 & 0.173 & - \\
\hline & L Ventricular Mass & -0.000001 & 0.000001 & 0.414 & - \\
\hline \multirow[t]{4}{*}{ Anterior CC } & Age & -0.000005 & 0.000002 & 0.033 & 0.675 \\
\hline & $\mathrm{VO}_{2 \max }$ & -0.000002 & 0.000002 & 0.249 & - \\
\hline & Body Mass Index & 0.000001 & 0.000004 & 0.872 & - \\
\hline & L Ventricular Mass & -0.000001 & 0.00001 & 0.433 & - \\
\hline
\end{tabular}

Note: all individuals were entered into regression, irrespective of group. L ventricular mass indexed to body surface area. Std: Standardized; L: left; R: right; CC: cingulate cortex. 
Table 3.4 Right hemisphere brain regions showing significant group differences in cortical thickness in a whole-brain analysis.

\begin{tabular}{|c|c|c|c|c|c|}
\hline $\begin{array}{c}\text { Right Hemisphere } \\
\text { Region }\end{array}$ & $\begin{array}{c}\text { Mean MA } \\
(\mathbf{m m})\end{array}$ & $\begin{array}{c}\text { Mean HA } \\
(\mathbf{m m})\end{array}$ & $\begin{array}{l}\text { Difference } \\
\text { (MA-HA) }\end{array}$ & StdError & t value \\
\hline Calcarine S & 3.1 & 2.6 & 0.5 & 0.13 & 3.80 \\
\hline Central S & 2.3 & 2.0 & 0.3 & 0.04 & 8.60 \\
\hline Cingulate $\mathrm{G}$ & 3.2 & 2.7 & 0.5 & 0.08 & 5.40 \\
\hline Cingulate $S$ & 2.7 & 2.4 & 0.3 & 0.04 & 7.30 \\
\hline Collateral S & 3.5 & 2.8 & 0.7 & 0.14 & 5.30 \\
\hline Cuneus & 2.4 & 2.0 & 0.4 & 0.06 & 6.40 \\
\hline Rectus G & 3.0 & 2.8 & 0.2 & 0.09 & 2.20 \\
\hline Inf FG & 2.7 & 2.4 & 0.3 & 0.06 & 5.70 \\
\hline Inf FS & 2.8 & 2.3 & 0.5 & 0.09 & 5.60 \\
\hline Inf OG & 3.1 & 2.4 & 0.7 & 0.12 & 6.00 \\
\hline Inf PL & 2.7 & 2.3 & 0.4 & 0.07 & 5.60 \\
\hline Inf TG & 3.5 & 2.9 & 0.6 & 0.09 & 7.10 \\
\hline Inf TS & 3.3 & 2.8 & 0.5 & 0.10 & 5.40 \\
\hline Insula & 3.4 & 2.7 & 0.7 & 0.07 & 9.00 \\
\hline Intraparietal S & 2.5 & 2.2 & 0.3 & 0.05 & 5.60 \\
\hline Lat OTG & 3.5 & 2.9 & 0.6 & 0.11 & 5.30 \\
\hline Lat $\mathrm{S}$ & 2.7 & 2.4 & 0.3 & 0.04 & 8.50 \\
\hline Med OTG & 2.8 & 2.3 & 0.5 & 0.10 & 5.20 \\
\hline Middle FG & 2.6 & 2.3 & 0.3 & 0.05 & 6.80 \\
\hline Middle OG & 2.9 & 2.3 & 0.6 & 0.09 & 6.50 \\
\hline Middle TG & 3.0 & 2.6 & 0.4 & 0.08 & 5.00 \\
\hline OTS & 3.5 & 2.9 & 0.6 & 0.14 & 4.20 \\
\hline Olfactory S & 3.9 & 2.5 & 1.4 & 0.24 & 5.90 \\
\hline Orbital G & 3.5 & 2.7 & 0.8 & 0.11 & 7.10 \\
\hline Orbital S & 3.2 & 2.5 & 0.7 & 0.10 & 6.70 \\
\hline Parahippocampal G & 4.1 & 3.3 & 0.8 & 0.18 & 4.30 \\
\hline POS & 2.5 & 2.2 & 0.3 & 0.05 & 7.30 \\
\hline Postcentral G & 2.2 & 1.9 & 0.3 & 0.04 & 7.80 \\
\hline Postcentral S & 2.4 & 2.1 & 0.3 & 0.05 & 6.70 \\
\hline Precentral G & 2.4 & 2.1 & 0.3 & 0.04 & 5.80 \\
\hline Precentral S & 2.6 & 2.2 & 0.4 & 0.05 & 7.20 \\
\hline Precuneus & 2.7 & 2.3 & 0.4 & 0.06 & 5.30 \\
\hline Sup FG & 2.7 & 2.5 & 0.2 & 0.04 & 5.20 \\
\hline Sup FS & 2.7 & 2.3 & 0.4 & 0.04 & 7.70 \\
\hline Sup OG & 2.8 & 2.2 & 0.6 & 0.10 & 6.10 \\
\hline Sup PL & 2.3 & 2.1 & 0.2 & 0.04 & 5.50 \\
\hline Sup TG & 2.7 & 2.5 & 0.2 & 0.05 & 4.40 \\
\hline Sup TS & 3.0 & 2.5 & 0.5 & 0.07 & 6.20 \\
\hline Supramarginal G & 2.7 & 2.4 & 0.3 & 0.05 & 6.00 \\
\hline Trans OS & 2.6 & 2.2 & 0.4 & 0.07 & 4.70 \\
\hline Med PreF & 3.9 & 3.1 & 0.8 & 0.17 & 4.50 \\
\hline
\end{tabular}

MA: masters athletes; HA: healthy active. S: sulcus; G: gyrus; Inf: inferior; Lat: lateral; Med: medial; Sup: superior; Trans: transverse; F: frontal; O: occipital; P: parietal; T: temporal, L: lobe. All p<0.005. 
Table 3.5 Left hemisphere brain regions showing significant group differences in cortical thickness in a whole-brain analysis.

\begin{tabular}{|c|c|c|c|c|c|}
\hline $\begin{array}{c}\text { Left Hemisphere } \\
\text { Region }\end{array}$ & $\begin{array}{c}\text { Mean MA } \\
(\mathbf{m m})\end{array}$ & $\begin{array}{c}\text { Mean HA } \\
(\mathbf{m m})\end{array}$ & $\begin{array}{c}\text { Difference } \\
\text { (MA-HA) }\end{array}$ & StdError & t value \\
\hline Calcarine $\mathrm{S}$ & 2.9 & 2.0 & 0.9 & 0.14 & 6.60 \\
\hline Central S & 3.2 & 1.8 & 1.4 & 0.16 & 8.80 \\
\hline Cingulate $\mathrm{G}$ & 3.9 & 2.7 & 1.2 & 0.14 & 8.60 \\
\hline Cingulate $\mathrm{S}$ & 3.8 & 2.4 & 1.4 & 0.22 & 6.30 \\
\hline Collateral S & 4.7 & 2.9 & 1.8 & 0.25 & 7.10 \\
\hline Cuneus & 4.3 & 2.1 & 2.2 & 0.37 & 6.00 \\
\hline Rectus G & 4.4 & 2.6 & 1.8 & 0.23 & 7.30 \\
\hline Inf FG & 2.7 & 2.2 & 0.5 & 0.08 & 6.30 \\
\hline Inf FS & 2.8 & 2.3 & 0.5 & 0.10 & 5.80 \\
\hline Inf OG & 3.0 & 2.2 & 0.8 & 0.14 & 5.60 \\
\hline Inf PL & 2.7 & 2.2 & 0.5 & 0.09 & 5.90 \\
\hline Inf TG & 4.2 & 2.9 & 1.3 & 0.23 & 6.10 \\
\hline Inf TS & 3.3 & 2.5 & 0.8 & 0.12 & 6.70 \\
\hline Insula & 3.2 & 2.2 & 1.0 & 0.10 & 9.70 \\
\hline Intraparietal S & 2.8 & 2.1 & 0.7 & 0.13 & 5.60 \\
\hline Lat OTG & 3.9 & 2.8 & 1.1 & 0.39 & 2.90 \\
\hline Lat $\mathrm{S}$ & 2.7 & 2.2 & 0.5 & 0.08 & 7.20 \\
\hline Med OTG & 2.6 & 1.8 & 0.8 & 0.12 & 6.00 \\
\hline Middle FG & 2.8 & 2.3 & 0.5 & 0.08 & 7.30 \\
\hline Middle OG & 3.0 & 1.9 & 1.1 & 0.18 & 6.40 \\
\hline Middle TG & 3.5 & 2.3 & 1.2 & 0.17 & 7.20 \\
\hline OTS & 4.2 & 2.6 & 1.6 & 0.39 & 4.00 \\
\hline Olfactory S & 3.6 & 2.6 & 1.0 & 0.62 & 1.70 \\
\hline Orbital G & 3.9 & 2.1 & 1.8 & 0.26 & 6.80 \\
\hline Orbital S & 3.7 & 2.7 & 1.0 & 0.18 & 5.40 \\
\hline Parahippocampal G & 4.0 & 3.2 & 0.8 & 0.58 & 1.40 \\
\hline POS & 2.5 & 2.0 & 0.5 & 0.08 & 5.70 \\
\hline Postcentral G & 2.7 & 2.1 & 0.6 & 0.06 & 9.70 \\
\hline Postcentral S & 2.5 & 1.9 & 0.6 & 0.09 & 6.50 \\
\hline Precentral G & 3.0 & 2.1 & 0.9 & 0.13 & 6.20 \\
\hline Precentral S & 2.8 & 2.2 & 0.6 & 0.08 & 6.60 \\
\hline Precuneus & 3.5 & 2.5 & 1.0 & 0.17 & 5.70 \\
\hline Sup FG & 3.3 & 2.4 & 0.9 & 0.08 & 10.00 \\
\hline Sup FS & 3.0 & 2.3 & 0.7 & 0.12 & 5.90 \\
\hline Sup OG & 3.0 & 1.9 & 1.1 & 0.18 & 6.40 \\
\hline Sup PL & 2.4 & 2.0 & 0.4 & 0.07 & 5.90 \\
\hline Sup TG & 2.8 & 2.2 & 0.6 & 0.10 & 6.00 \\
\hline Sup TS & 3.8 & 2.3 & 1.5 & 0.22 & 6.70 \\
\hline Supramarginal G & 2.6 & 2.1 & 0.5 & 0.09 & 5.70 \\
\hline Trans OS & 3.3 & 2.2 & 1.1 & 0.18 & 5.90 \\
\hline Med PreF & 3.9 & 2.8 & 1.1 & 0.20 & 5.60 \\
\hline
\end{tabular}

MA: masters athletes; HA: healthy active. S: sulcus; G: gyrus; Inf: inferior; Lat: lateral; Med: medial; Sup: superior; Trans: transverse; F: frontal; O: occipital; P: parietal; T: temporal, L: lobe. All $\mathrm{p}<0.005$. 
Table 3.6 Results of multiple linear regression of ROI cortical thickness against anthropometric covariates.

\begin{tabular}{|c|c|c|c|c|c|}
\hline & & Std. Coefficient & Std. Error & $P$ value & Power $(\alpha 0.05)$ \\
\hline \multirow[t]{4}{*}{ R Insula } & Age & -0.0014 & 0.008 & 0.862 & - \\
\hline & $\mathrm{VO}_{2 \text { max }}$ & 0.0169 & 0.007 & 0.031 & 0.999 \\
\hline & Body Mass Index & -0.0116 & 0.015 & 0.453 & - \\
\hline & L Ventricular Mass & 0.0016 & 0.004 & 0.682 & - \\
\hline \multirow[t]{4}{*}{ L Insula } & Age & 0.0009 & 0.011 & 0.935 & - \\
\hline & $\mathrm{VO}_{2 \text { max }}$ & 0.0318 & 0.010 & 0.005 & 0.999 \\
\hline & Body Mass Index & 0.0024 & 0.021 & 0.911 & - \\
\hline & L Ventricular Mass & -0.0035 & 0.005 & 0.515 & - \\
\hline \multirow[t]{4}{*}{ R Med PreF } & Age & -0.0008 & 0.019 & 0.968 & - \\
\hline & $\mathrm{VO}_{2 \text { max }}$ & 0.0353 & 0.018 & 0.047 & 0.973 \\
\hline & Body Mass Index & 0.0198 & 0.036 & 0.591 & - \\
\hline & L Ventricular Mass & 0.0001 & 0.009 & 0.991 & - \\
\hline \multirow[t]{4}{*}{ L Med PreF } & Age & 0.0134 & 0.009 & 0.133 & - \\
\hline & $\mathrm{VO}_{2 \text { max }}$ & 0.0227 & 0.008 & 0.008 & 0.999 \\
\hline & Body Mass Index & -0.0031 & 0.016 & 0.852 & - \\
\hline & L Ventricular Mass & 0.0070 & 0.004 & 0.096 & - \\
\hline \multirow[t]{4}{*}{ R Precentral } & Age & 0.0007 & 0.006 & 0.903 & - \\
\hline & $\mathrm{VO}_{2 \text { max }}$ & 0.0044 & 0.005 & 0.420 & - \\
\hline & Body Mass Index & -0.0094 & 0.011 & 0.406 & - \\
\hline & L Ventricular Mass & 0.0006 & 0.003 & 0.829 & - \\
\hline \multirow[t]{4}{*}{ L Precentral } & Age & -0.0002 & 0.004 & 0.964 & - \\
\hline & $\mathrm{VO}_{2 \text { max }}$ & 0.0087 & 0.004 & 0.035 & 0.993 \\
\hline & Body Mass Index & 0.0008 & 0.008 & 0.920 & - \\
\hline & L Ventricular Mass & -0.0033 & 0.002 & 0.117 & - \\
\hline \multirow[t]{4}{*}{ R Postcentral } & Age & 0.0019 & 0.004 & 0.647 & - \\
\hline & $\mathrm{VO}_{2 \text { max }}$ & 0.0083 & 0.004 & 0.039 & 0.998 \\
\hline & Body Mass Index & -0.0097 & 0.008 & 0.230 & - \\
\hline & L Ventricular Mass & -0.0013 & 0.002 & 0.528 & - \\
\hline \multirow[t]{4}{*}{ L Postcentral } & Age & 0.0041 & 0.005 & 0.374 & - \\
\hline & $\mathrm{VO}_{2 \text { max }}$ & 0.0064 & 0.004 & 0.140 & - \\
\hline & Body Mass Index & -0.0069 & 0.009 & 0.434 & - \\
\hline & L Ventricular Mass & 0.0009 & 0.002 & 0.687 & - \\
\hline
\end{tabular}

Note: all individuals were entered into regression, irrespective of group. L ventricular mass indexed to body surface area. Std: Standardized; L: left; R: right. 
Figure 3.1 Regression analysis of region-of-interest subcortical volumes with age.
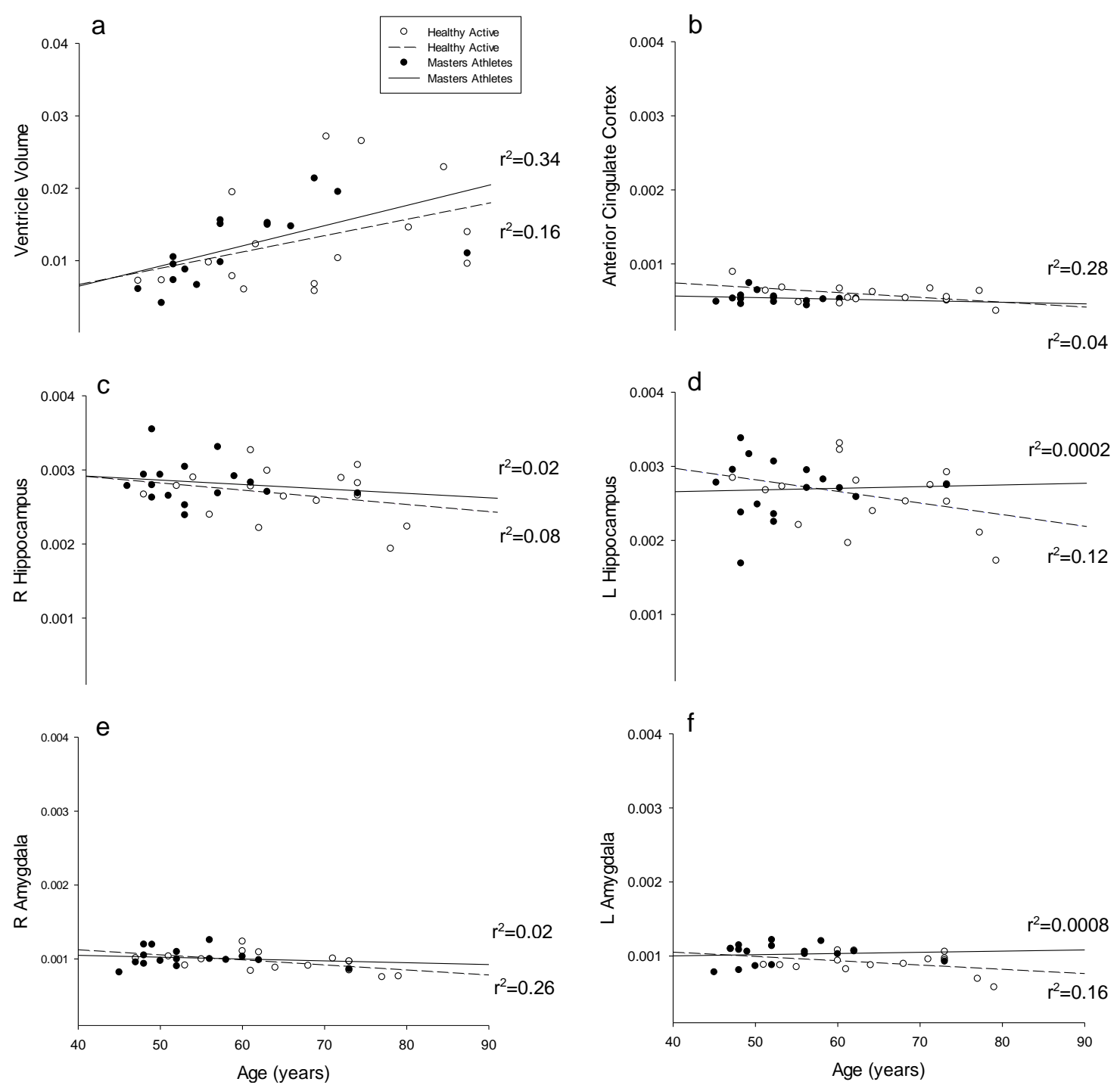

Slope of regression was not different between groups for (a) lateral ventricle volume (probability $=0.75$ ); (b) anterior cingulate cortex (probability $=0.31$ ); (c) right hippocampus (probability=0.57); (d) left hippocampus (probability=0.25); (e) right amygdala (probability $=0.44$ ); and (f) left amygdala (probability $=0.11$ ). All volumes are indexed to total intracranial volume and measured in $\mathrm{mm}^{3}$. 
Figure 3.2 Group differences in cortical thickness (MA > HA).

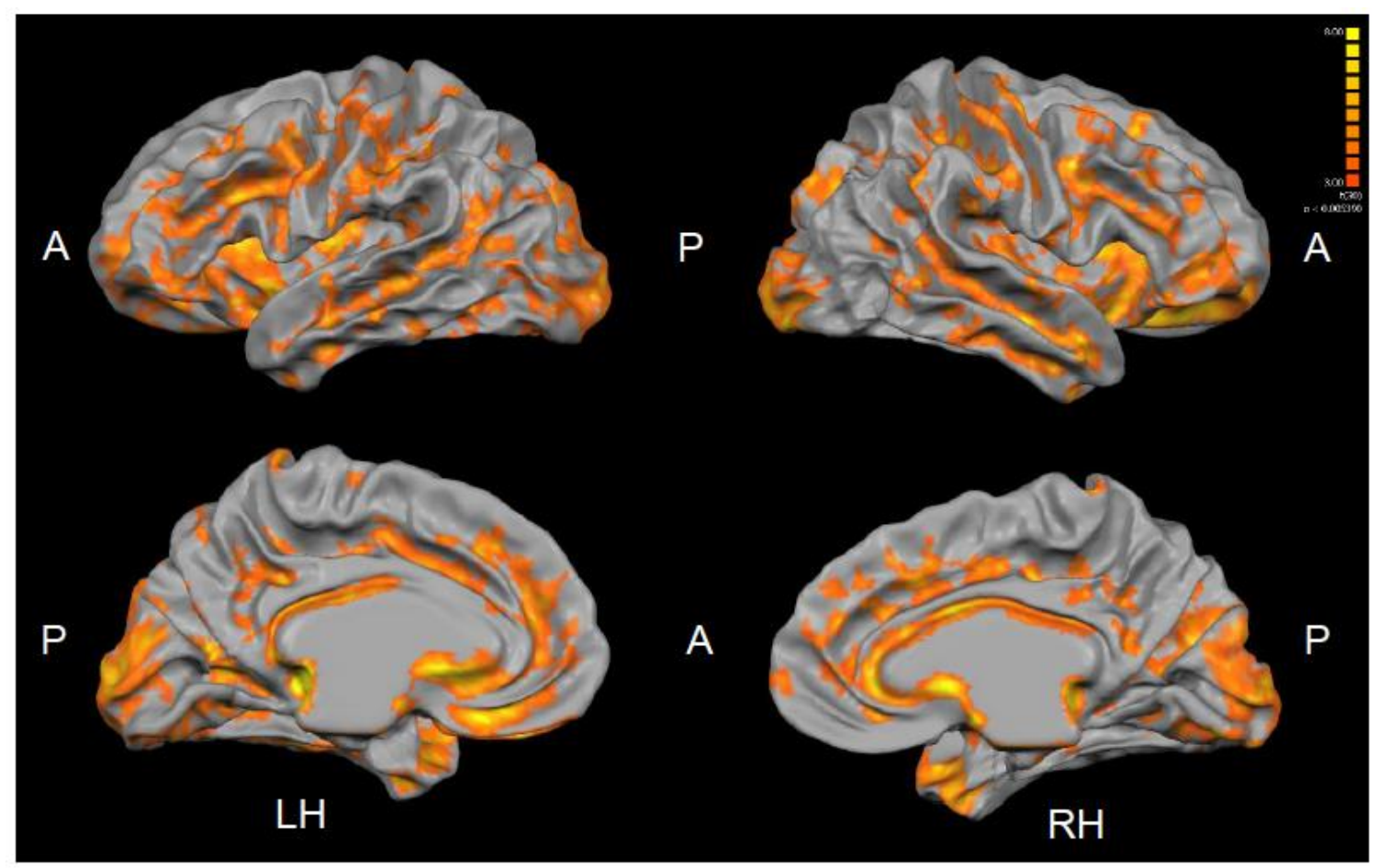

Results are shown at $\mathrm{p}<0.005$, corrected by cluster size $\left(10 \mathrm{~mm}^{2}\right)$. Color scale at right denotes t values. LH: left hemisphere; RH: right hemisphere; A: anterior; P: posterior. Many regions in MA were significantly thicker than HA subjects (see Tables 3.4-3.5). 
Figure 3.3 Regression analysis of region-of-interest cortical thickness with age.
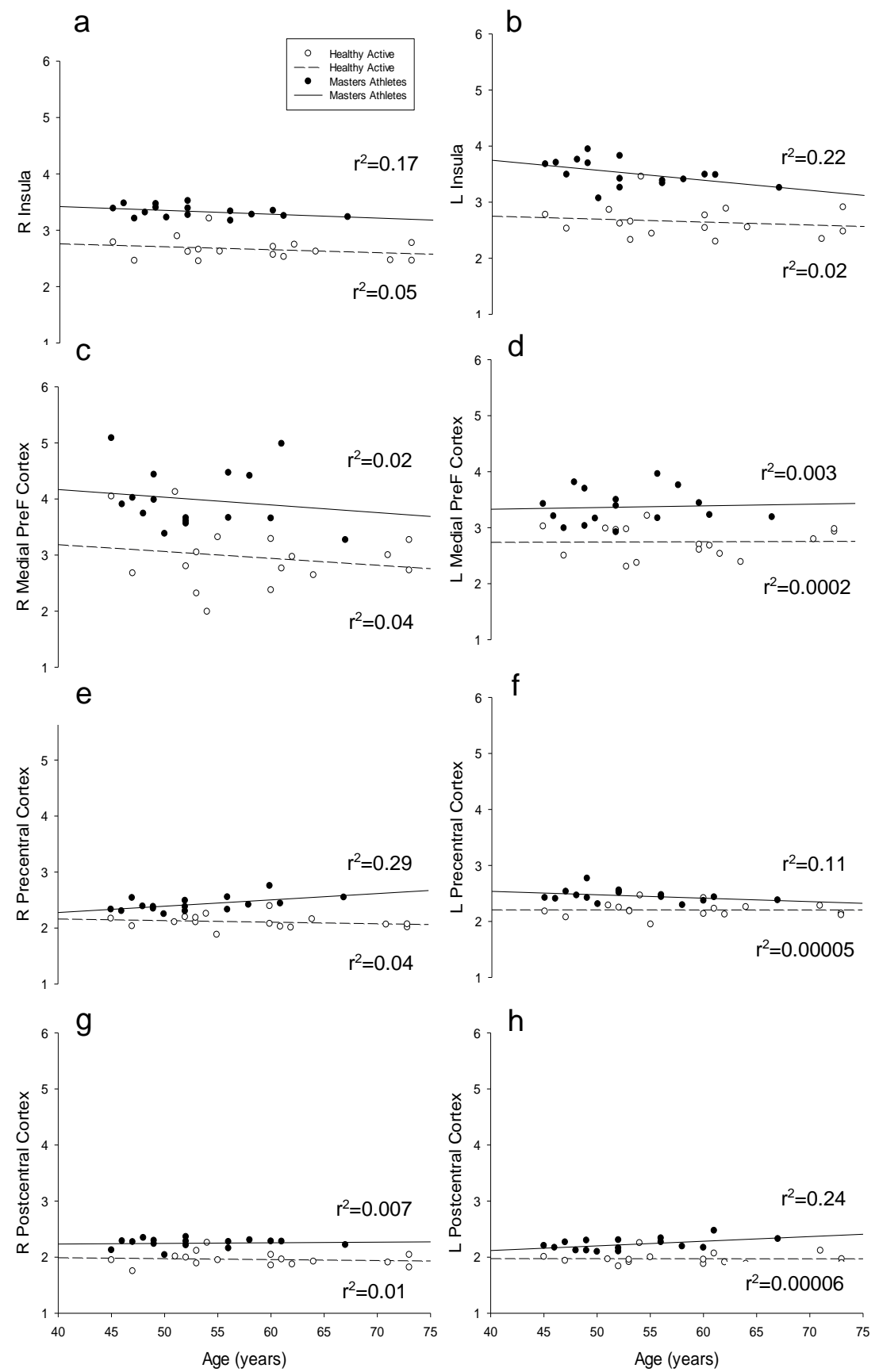

Slope of regression was not different between groups for (a) right insula ( $\mathrm{P}=0.82)$; (b) left insula ( $\mathrm{P}=0.32)$; (c) right medial prefrontal cortex $(\mathrm{P}=0.96)$; (d) left medial prefrontal cortex $(\mathrm{P}=0.88)$; (f) left precentral gyrus $(\mathrm{P}=0.33)$; $(\mathrm{g})$ right postcentral gyrus $(\mathrm{P}=0.95)$; and $(\mathrm{h})$ left postcentral gyrus $(\mathrm{P}=0.11)$. Masters athletes had a different trajectory with age at $(\mathrm{e})$ right precentral gyrus $(\mathrm{P}=0.02)$. Cortical thickness measured in $\mathrm{mm}$. 
Figure 3.4 Full cortex correlation analysis with age covariate.

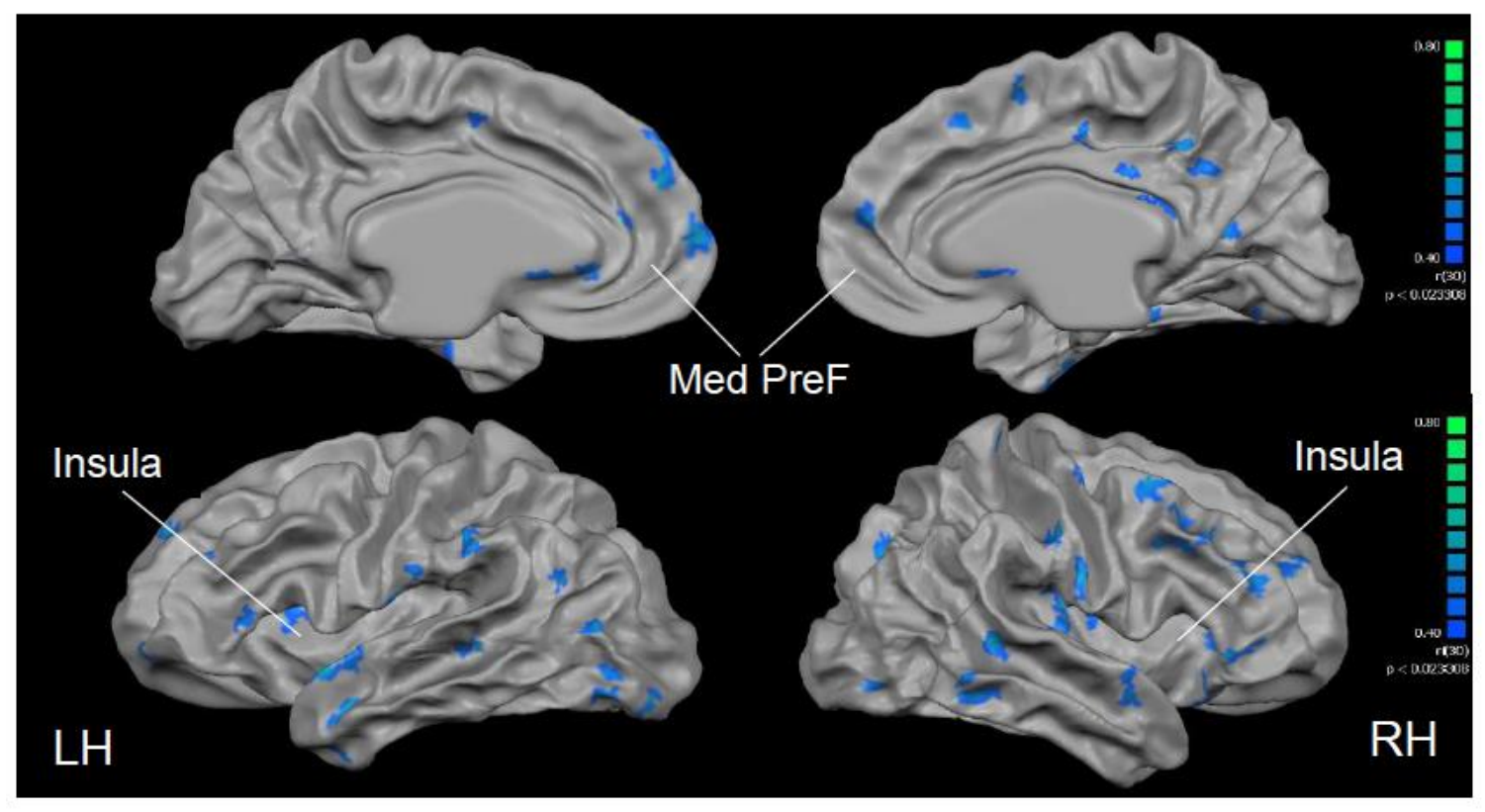

All subjects, irrespective of group. Results are shown at $\mathrm{p}<0.05$, corrected by cluster size $\left(10 \mathrm{~mm}^{2}\right)$. Color scale at right denotes $\mathrm{t}$ values. LH: left hemisphere; RH: right hemisphere; Med: medial; PreF: prefrontal. Regions of interest are labeled. 
Figure 3.5 Full cortex negative correlation analysis with $\mathrm{VO}_{2 \max }$ covariate.

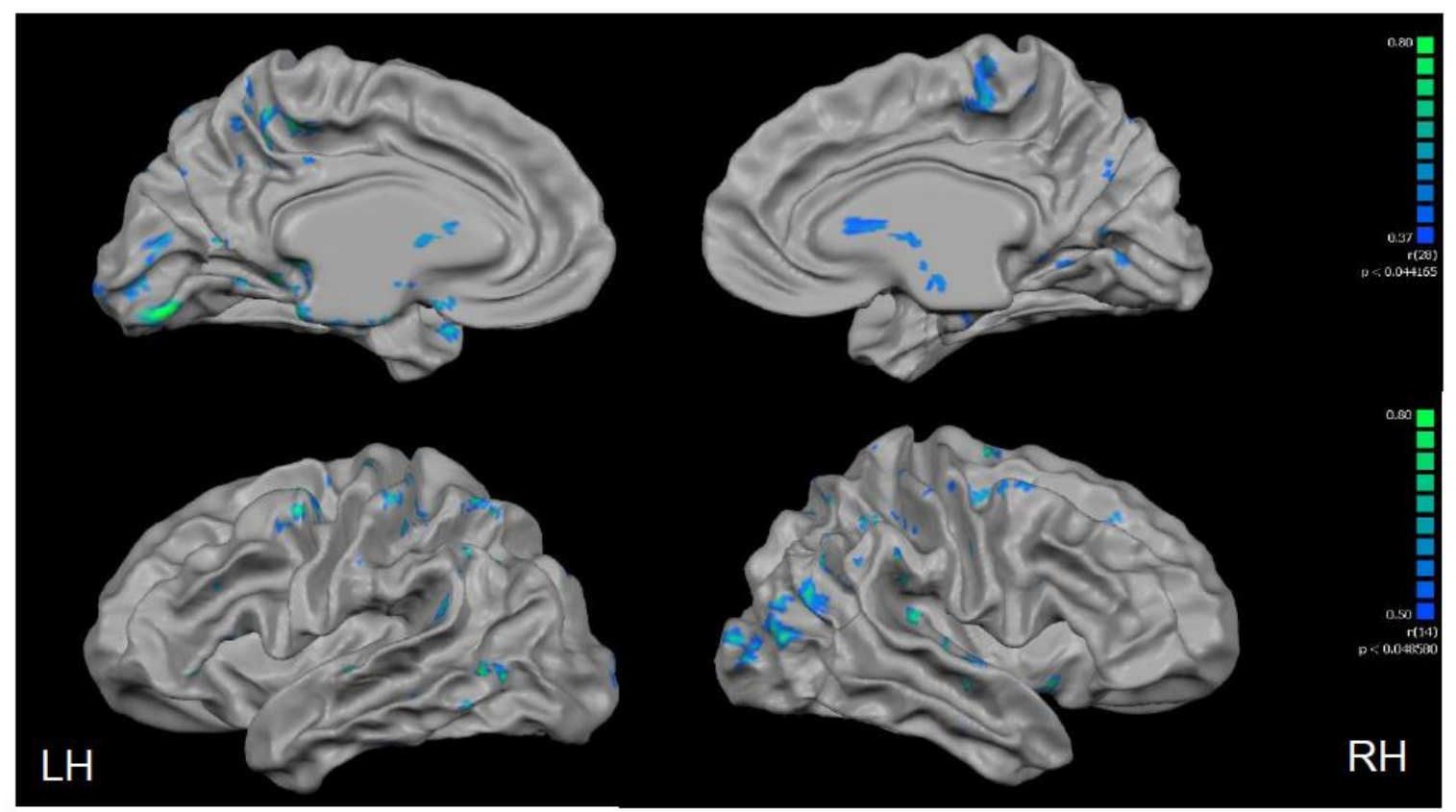

All subjects, irrespective of group. Results are shown at $\mathrm{p}<0.005$, corrected by cluster size $\left(10 \mathrm{~mm}^{2}\right)$. Color scale at right denotes $\mathrm{t}$ values. LH: left hemisphere; RH: right hemisphere. 
Figure 3.6
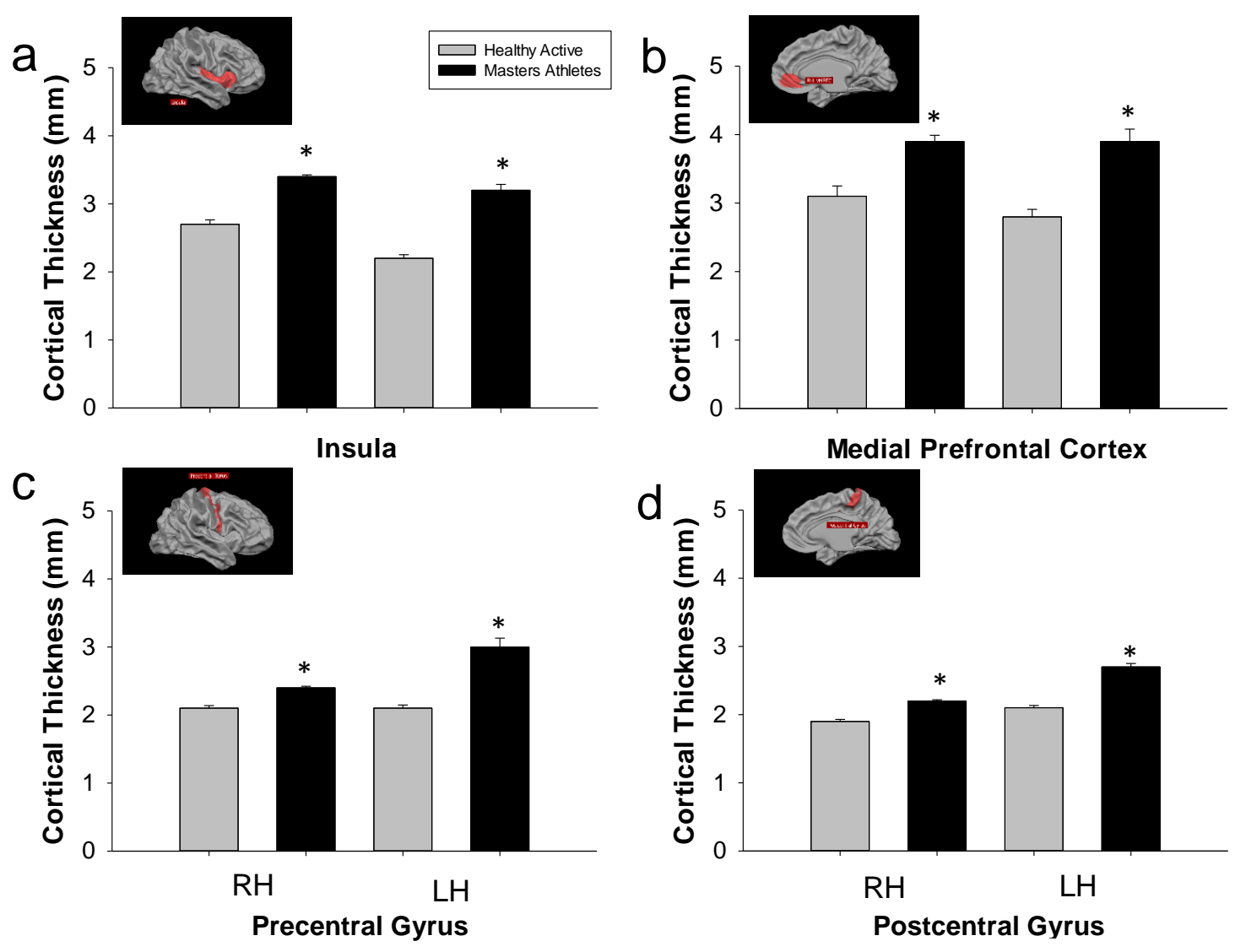

Cortical thickness at anatomically defined regions of interest in insula (a), medial prefrontal cortex (b), precentral gyrus (c), and postcentral gyrus (d). HA (gray bars), MA (black bars). RH: right hemisphere (left side pair of bars); LH: left hemisphere (right side pair of bars). * Different from HA, p<0.0005, corrected by cluster size $\left(10 \mathrm{~mm}^{2}\right)$. 
Figure 3.7 Full cortex correlation analysis with $\mathrm{VO}_{2 \max }$ covariate.

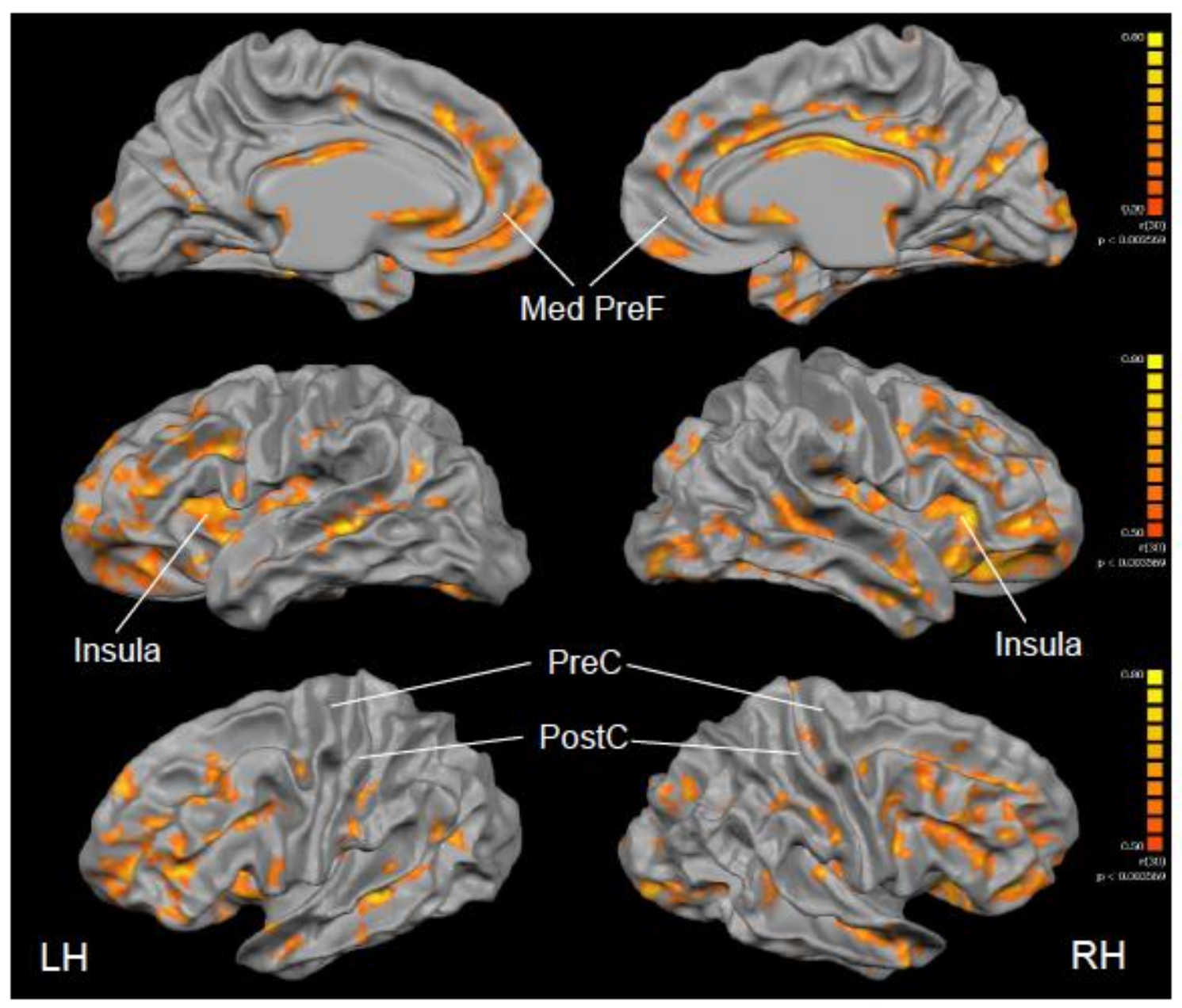

All subjects, irrespective of group. Results are shown at $\mathrm{p}<0.005$, corrected by cluster size $\left(10 \mathrm{~mm}^{2}\right)$. Color scale at right denotes $t$ values. $\mathrm{LH}$ : left hemisphere; RH: right hemisphere; Med: medial; PreF: prefrontal; C: central. Regions of interest are labeled. 


\subsection{Discussion}

This study examined the benefit that high levels of exercise training and cardiorespiratory fitness could have upon brain structure in a healthy human model. Overall, long-term training and high levels of cardiorespiratory fitness, elicited and sustained, greater cortical thickness over a large portion of the brain in MA than HA groups. However, lifelong exercise training had little effect on the rate of age-related cortical atrophy and, had no effect on subcortical structures relative to that of modestly active adults. In addition, with the possible exception of focal regions of cortical thinning in the occipital and parietal lobes, life-long, high-intensity exercise training did not produce any apparent detrimental effect as indicated by greater total grey matter in MA, equivalent white matter hypointensities, and normal blood-borne inflammatory-markers, when compared to age-matched healthy active counterparts. Therefore, the current results illustrate the potent effect of this very high exercise stimulus to develop cortical (but not subcortical) reserve at younger ages, and sustain this reserve into the senior years. These data suggest that chronic endurance training provides a benefit in cortical neural reserve beyond that provided by guideline-based activity, but does not eliminate the effect of age on cortical grey matter atrophy.

The current study is the first to present evidence that life-long aerobic training, initiated early in life and sustained over decades, has a significant impact on total grey matter, and whole-brain cortical mass. Middle to older-aged MA were chosen for this study as they represent a dedicated athletic career lasting, in some cases, longer than 30 years, a proven athletic performance background, and a high to very high age-adjusted $\mathrm{VO}_{2 \max }(\sim 125 \%$ of age-predicted maximum). Therefore, a reasonable conjecture is that these individuals represent the highest expected level of exercise training in aging adults and, therefore, provide an index of the potential to grow and preserve brain mass across the age span. Similarly, all HA participants were chosen based on their self-reported history of adherence to a healthy active lifestyle ( $>5$ years) that met the age-adjusted physical activity guidelines (ACSM 1995). These guidelines emphasize 30 minutes of moderate intensity exercise on five or more days of the week, confirmed by an age-adjusted $\mathrm{VO}_{2 \max }$ of nearly $100 \%$. In addition, our HA sample had normal weight and BMI scores, and 
hemodynamic values statistically the same to those of MA and all within normal limits. Therefore, all participants in the current study were free of concerns related to sedentary living such as medications, brain pathology and cognitive dysfunction, and represent a model of active, successful aging (Thielke and Diehr 2012). Therefore, we propose that the current data represent a specific effect of exercise training.

\subsubsection{Subcortical Gray Matter and Lateral Ventricular Volume}

An interplay between subcortical mass and cerebral spinal fluid volume often results in the passive expansion of the lateral ventricles as neighboring grey matter mass declines (Weuve, Kang et al. 2004, Podewils, Guallar et al. 2005). Numerous cross-sectional imaging studies highlight the reduction in hippocampus (HC) volume with age, accompanied by a significant increase in ventricular volume (Gur, Mozley et al. 1991, Bhatia, Bookheimer et al. 1993, Blatter, Bigler et al. 1995, Forstl, Zerfass et al. 1995, Jack, Petersen et al. 1997, Mu, Xie et al. 1999, Jack, Knopman et al. 2013, Cash, Frost et al. 2015). However, contradictory data also exist regarding age (Mueller, Moore et al. 1998, Resnick, Goldszal et al. 2000) and HC volume (Jack, Twomey et al. 1989, Ohnishi, Matsuda et al. 2001). The current observations support the idea that age predicts lateral ventricular and $\mathrm{HC}$ volumes. However, Pearson correlation analysis revealed that ventricular volume also correlated weakly and inversely with $\mathrm{VO}_{2 \max }(\mathrm{r}=-0.28, \mathrm{p}=$ 0.05), due possibly to the expansion of the cerebral cortex space in the MA group, discussed below.

The HC is possibly the most commonly researched brain structure in the context of adaptations to exercise training. For example, Erickson et al. illustrate rapid expansion of the HC volume within 6 weeks of the onset of exercise training in older sedentary adults with a baseline state of cognitive impairment (Erickson, Voss et al. 2011). This conclusion contrasts with the current observations that indicate little association between cardiorespiratory fitness and $\mathrm{HC}$ volume. However, the current participants were all healthy, in the middle age range, and were at, or above, their age-predicted level of

cardiorespiratory fitness for active individuals. Therefore, the potential capacity for $\mathrm{HC}$ volumetric changes in response to exercise are likely a function of the starting baseline 
condition. In the current study, we suspect the lack of $\mathrm{HC}$ volumetric change between groups was due to a maximal effect achieved by guideline-based exercise training.

In contrast to the hippocampus, left amygdala volumes were greater in MA versus HA groups and were related to $\mathrm{VO}_{2 \max }$. The specific benefit of this adaptive response to lifelong training could be multifactorial, given the role of this region in the processing of emotion, learning, memory, and autonomic cardiovascular adjustments.

\subsubsection{Cortical Gray Matter}

In contrast to the subcortical gray matter, we observed a widespread enhancement of cerebral cortex grey matter thickness in the MA group compared to the HA group. The impact of training in MA varied across the brain with some regions expressing no benefit while others indicated approximately $25 \%$ greater cortical thickness. These latter regions were evident in the frontal lobe, temporal lobe, insula cortex, parietal lobe and occipital regions. Thus, marked cortical "reserve" was evident in these regions across the entire age span including the middle age stages where the duration of training was less than those in the higher ages.

As mentioned, a notable outcome of the current study was the widespread cortical thickening in MA versus HA. Our whole brain analysis (Tables 3.4 and 3.5) reveals a number of cortical sites including the temporal and occipital lobes. Previously, Tseng et al. (Tseng, Uh et al. 2013) reported the confinement of cortical benefits of training in master's athletes to the posterior portion of the brain in the cuneus and precuneus. The current study differs from the previous Tseng (Tseng, Uh et al. 2013) study in the observation of widespread cortical thickening in MA. A wider age range and larger sample size may factor in these between-study differences. Nonetheless, the current results support those of Tseng et al. (Tseng, Uh et al. 2013) in the observation of greater cortical thickness in the cuneus and precuneus when compared to a sedentary elderly group. The possible implications of these observations are not clear but may relate to the functional role of the default mode network in neurocognitive and autonomic processing (Utevsky, Smith et al. 2014). 
Further, the current data along with those of Tseng et al. (Tseng, Uh et al. 2013), give rise to a possible outcome that the posterior brain not only exhibits resistance to age-related atrophy but also demonstrates sensitivity to exercise stimuli across the age span studied here. Overall, the current observations support a speculation that the additional cortical reserve achieved early in life through high cardiorespiratory fitness will minimize the risk for neurological impairment in senescence and reduce the frailty period of life.

The mechanisms that operate to enhance cortical structure with training remain unknown and are likely multifactorial. In general, axonal, dendritic, and glial processes account for $\sim 50 \%$ of grey matter volume whereas vascular (5\%), other cell types (20\%) and interstitial space (cerebrospinal fluid) account for the remainder (Thomas, Dennis et al. 2012). Recent evidence highlights the benefits of exercise training on pial vessel number, synaptogenesis (Cotman and Berchtold 2002), neurogenesis (van Praag, Christie et al. 1999) and astrocyte expansion (Saur, Baptista et al. 2014). To account for the regional $\sim 25 \%$ greater cortical thickness in the MA group, some combination of expansion in any or all of these tissue types may be required.

\subsubsection{Negative Association with High Fitness and Brain Structure}

Chronic exposure to stress, whether it occurs during childhood, adolescence, adulthood or aging, has a negative impact on brain structure (Sapolsky 1999, Landfield, Blalock et al. 2007, Lupien, McEwen et al. 2009). Freund et al. (Freund, Faust et al. 2012) illustrated the significant, but reversible, reduction in brain volume following the TransEurope footrace that occurs over several weeks. The MA in our study were training $>15 \mathrm{~h} / \mathrm{wk}$ for decades which may amount to a significant level of physical stress. Therefore, concerns arise regarding the potential damage that intensive long-term competitive events may present to the brain, possibly due to persistent inflammatory mechanisms. However, no differences in white matter hypointensities, chronic inflammatory status (i.e., hsCRP levels), or altered lipidemic or glucogenic stress existed between groups. Therefore, little detrimental effect of chronic competitive training on brain structure occurred in the MA. In contrast, Tseng et al. (Tseng, Uh et al. 2013) observed an $83 \%$ reduction in white matter hypointensities in MA compared to sedentary adults. We attribute this discrepancy to the fact that our HA group was not sedentary and thus preserved white matter integrity 
equally as well as our MA population. If so, then guideline-based training may be sufficient for maximal preservation of white matter integrity and subcortical structure volumes to the same degree as high-intensity long duration training. We did observe isolated regions of cortical thinning in MA relative to HA, located within the parietal and occipital regions. However, whether this observation reflects variations in regional adaptability or cortical damage remains unknown. It is noteworthy, however, that none of the MA group reported neurological cardiovascular problems such as fibrillation, and the regions of thinner cortex in these athletes have not been associated with autonomic cardiovascular function.

\subsection{Limitations}

Significant limitations of this study include lack of data on the proportional contributions of the exercise effect versus the environmental, genetic, or social situations on brain structure, that likely change over time.

We acknowledge the small sample size of the current groups and promote caution in interpreting the results. Nonetheless, using a random selection of $75 \%$ of our sample indicated that $\mathrm{VO}_{2 \max }$ remained the strongest predictor of cortical thickness and regionof-interest subcortical volume. We have also used a rigorous threshold protocol for the voxel-wise analysis $\left(\mathrm{p}<0.005\right.$, cluster size $\left.>10 \mathrm{~mm}^{2}\right)$ to reduce the probability of false findings which could be associated with a small sample size.

Our study may also be limited by the age-range of our participants being 45-73 years in HA and 45-67 years in MA. This age range does not reflect the older age range in which cortical atrophy becomes more apparent.

Finally, we acknowledge the limitations of the cognitive testing protocol used in this study. We did not attempt to complete a comprehensive analysis of cognitive function. Rather, we collected MoCA and Trail Making questionnaires as a rapid but valid estimate of overall cognitive health. 


\subsection{Conclusion}

We present novel findings that sustained high levels of cardiorespiratory fitness increase cortical thickness in many brain regions. However, the impact on subcortical grey matter appears to peak at levels of activity that meet the published guidelines for adults of middle to older age. Nonetheless, despite producing greater cerebral cortex thickness, the MA training load had no effect on the trajectory of age-related cortical atrophy. In fact, although based on small sample sizes, the rates of decline in the selected cortical regions of interest in the current study fall within the expected $0-0.5 \%$ range for this age group (Salat, Buckner et al. 2004, Raz, Lindenberger et al. 2005), in each of the MA and HA groups. Therefore, the benefits of life-long exercise training were limited to the expression and sustainment of cortical reserve, but not age-related decline. Therefore, in cognitively normal, healthy individuals, the neurological benefit of chronic training appears to include a sustained cortical reserve, producing greater cortical mass and possibly improved neurological function at every age. Furthermore, life-long, highintensity exercise did not produce a notable detrimental impact on brain structure. The expectations of these observations are that the $25 \%$ reserve in cortical thickness that accompanied lifelong training will preserve neurologic function into the senescent years. 


\subsection{References}

ACSM (1995). Guidelines for Exercise Testing and Prescription. L. W. a. Wilkins. Philadelphia: 160.

Angevaren, M., G. Aufdemkampe, H. J. Verhaar, A. Aleman and L. Vanhees (2008). "Physical activity and enhanced fitness to improve cognitive function in older people without known cognitive impairment." Cochrane Database Syst Rev(3): CD005381.

Barberi, E. A., J. S. Gati, B. K. Rutt and R. S. Menon (2000). "A transmit-only/receiveonly (TORO) RF system for high-field MRI/MRS applications." $\underline{\text { Magn Reson. Med }}$ 43(2): 284-289.

Barnes, D. E., K. Yaffe, W. A. Satariano and I. B. Tager (2003). "A longitudinal study of cardiorespiratory fitness and cognitive function in healthy older adults." J Am Geriatr Soc 51(4): 459-465.

Barry, V. W., M. Baruth, M. W. Beets, J. L. Durstine, J. Liu and S. N. Blair (2014). "Fitness vs. fatness on all-cause mortality: a meta-analysis." Prog Cardiovasc Dis 56(4): 382-390.

Bhatia, S., S. Y. Bookheimer, W. D. Gaillard and W. H. Theodore (1993). "Measurement of whole temporal lobe and hippocampus for MR volumetry: normative data." Neurology 43(10): 2006-2010.

Blatter, D. D., E. D. Bigler, S. D. Gale, S. C. Johnson, C. V. Anderson, B. M. Burnett, N. Parker, S. Kurth and S. D. Horn (1995). "Quantitative volumetric analysis of brain MR: normative database spanning 5 decades of life." AJNR Am J Neuroradiol 16(2): 241-251.

Booth, F. W., C. K. Roberts and M. J. Laye (2012). "Lack of exercise is a major cause of chronic diseases." Compr Physiol 2(2): 1143-1211.

Cash, D. M., C. Frost, L. O. Iheme, D. Unay, M. Kandemir, J. Fripp, O. Salvado, P. Bourgeat, M. Reuter, B. Fischl, M. Lorenzi, G. B. Frisoni, X. Pennec, R. K. Pierson, J. L. Gunter, M. L. Senjem, C. R. Jack, Jr., N. Guizard, V. S. Fonov, D. L. Collins, M. Modat, 
M. J. Cardoso, K. K. Leung, H. Wang, S. R. Das, P. A. Yushkevich, I. B. Malone, N. C. Fox, J. M. Schott and S. Ourselin (2015). "Assessing atrophy measurement techniques in dementia: Results from the MIRIAD atrophy challenge." Neuroimage 123: 149-164.

Chaddock, L., K. I. Erickson, R. S. Prakash, M. W. Voss, M. VanPatter, M. B. Pontifex, C. H. Hillman and A. F. Kramer (2012). "A functional MRI investigation of the association between childhood aerobic fitness and neurocognitive control." Biol Psychol 89(1): 260-268.

Coffey, C. E., W. E. Wilkinson, I. A. Parashos, S. A. Soady, R. J. Sullivan, L. J. Patterson, G. S. Figiel, M. C. Webb, C. E. Spritzer and W. T. Djang (1992). "Quantitative cerebral anatomy of the aging human brain: a cross-sectional study using magnetic resonance imaging." Neurology 42 (3 Pt 1): 527-536.

Cotman, C. W. and N. C. Berchtold (2002). "Exercise: a behavioral intervention to enhance brain health and plasticity." Trends Neurosci 25(6): 295-301.

Dale, A. M., B. Fischl and M. I. Sereno (1999). "Cortical surface-based analysis. I. Segmentation and surface reconstruction." Neuroimage 9(2): 179-194.

Dale, A. M. and M. I. Sereno (1993). "Improved Localizadon of Cortical Activity by Combining EEG and MEG with MRI Cortical Surface Reconstruction: A Linear Approach." J Cogn Neurosci 5(2): 162-176.

Debette, S. and H. S. Markus (2010). "The clinical importance of white matter hyperintensities on brain magnetic resonance imaging: systematic review and metaanalysis." BMJ 341: c3666.

Defina, L. F., B. L. Willis, N. B. Radford, A. Gao, D. Leonard, W. L. Haskell, M. F. Weiner and J. D. Berry (2013). "The association between midlife cardiorespiratory fitness levels and later-life dementia: a cohort study." Ann Intern Med 158(3): 162-168.

Du, A. T., N. Schuff, J. H. Kramer, H. J. Rosen, M. L. Gorno-Tempini, K. Rankin, B. L. Miller and M. W. Weiner (2007). "Different regional patterns of cortical thinning in Alzheimer's disease and frontotemporal dementia." Brain 130(Pt 4): 1159-1166. 
Erickson, K. I., C. A. Raji, O. L. Lopez, J. T. Becker, C. Rosano, A. B. Newman, H. M. Gach, P. M. Thompson, A. J. Ho and L. H. Kuller (2010). "Physical activity predicts gray matter volume in late adulthood: the Cardiovascular Health Study." Neurology 75(16): 1415-1422.

Erickson, K. I., M. W. Voss, R. S. Prakash, C. Basak, A. Szabo, L. Chaddock, J. S. Kim, S. Heo, H. Alves, S. M. White, T. R. Wojcicki, E. Mailey, V. J. Vieira, S. A. Martin, B. D. Pence, J. A. Woods, E. McAuley and A. F. Kramer (2011). "Exercise training increases size of hippocampus and improves memory." Proc Natl Acad Sci U S A 108(7): 3017-3022.

Etnier, J. L., P. M. Nowell, D. M. Landers and B. A. Sibley (2006). "A meta-regression to examine the relationship between aerobic fitness and cognitive performance." Brain $\underline{\operatorname{Res} \operatorname{Rev}}$ 52(1): 119-130.

Fischl, B. and A. M. Dale (2000). "Measuring the thickness of the human cerebral cortex from magnetic resonance images." Proc Natl Acad Sci U S A 97(20): 11050-11055.

Fischl, B., A. Liu and A. M. Dale (2001). "Automated manifold surgery: constructing geometrically accurate and topologically correct models of the human cerebral cortex." IEEE Trans Med Imaging 20(1): 70-80.

Fischl, B., D. H. Salat, E. Busa, M. Albert, M. Dieterich, C. Haselgrove, A. van der Kouwe, R. Killiany, D. Kennedy, S. Klaveness, A. Montillo, N. Makris, B. Rosen and A. M. Dale (2002). "Whole brain segmentation: automated labeling of neuroanatomical structures in the human brain." Neuron 33(3): 341-355.

Fischl, B., D. H. Salat, A. J. van der Kouwe, N. Makris, F. Segonne, B. T. Quinn and A. M. Dale (2004). "Sequence-independent segmentation of magnetic resonance images." Neuroimage 23 Suppl 1: S69-84.

Forstl, H., R. Zerfass, C. Geiger-Kabisch, H. Sattel, C. Besthorn and F. Hentschel (1995). "Brain atrophy in normal ageing and Alzheimer's disease. Volumetric discrimination and clinical correlations." Br J Psychiatry 167(6): 739-746. 
Freund, W., S. Faust, F. Birklein, C. Gaser, A. P. Wunderlich, M. Muller, C. Billich, M. S. Juchems, B. L. Schmitz, G. Gron and U. H. Schutz (2012). "Substantial and reversible brain gray matter reduction but no acute brain lesions in ultramarathon runners: experience from the TransEurope-FootRace Project." BMC Med 10: 170.

Fujimoto, N., A. Prasad, J. L. Hastings, A. Arbab-Zadeh, P. S. Bhella, S. Shibata, D. Palmer and B. D. Levine (2010). "Cardiovascular effects of 1 year of progressive and vigorous exercise training in previously sedentary individuals older than 65 years of age." Circulation 122(18): 1797-1805.

Goebel, R., F. Esposito and E. Formisano (2006). "Analysis of functional image analysis contest (FIAC) data with brainvoyager QX: From single-subject to cortically aligned group general linear model analysis and self-organizing group independent component analysis." Hum. Brain Mapp 27(5): 392-401.

Good, C. D., I. S. Johnsrude, J. Ashburner, R. N. Henson, K. J. Friston and R. S. Frackowiak (2001). "A voxel-based morphometric study of ageing in 465 normal adult human brains." Neuroimage 14(1 Pt 1): 21-36.

Gur, R. C., P. D. Mozley, S. M. Resnick, G. L. Gottlieb, M. Kohn, R. Zimmerman, G. Herman, S. Atlas, R. Grossman, D. Berretta and et al. (1991). "Gender differences in age effect on brain atrophy measured by magnetic resonance imaging." Proc Natl Acad Sci U S A 88(7): 2845-2849.

Jack, C. R., Jr., D. S. Knopman, W. J. Jagust, R. C. Petersen, M. W. Weiner, P. S. Aisen, L. M. Shaw, P. Vemuri, H. J. Wiste, S. D. Weigand, T. G. Lesnick, V. S. Pankratz, M. C. Donohue and J. Q. Trojanowski (2013). "Tracking pathophysiological processes in Alzheimer's disease: an updated hypothetical model of dynamic biomarkers." Lancet Neurol 12(2): 207-216.

Jack, C. R., Jr., R. C. Petersen, Y. C. Xu, S. C. Waring, P. C. O'Brien, E. G. Tangalos, G. E. Smith, R. J. Ivnik and E. Kokmen (1997). "Medial temporal atrophy on MRI in normal aging and very mild Alzheimer's disease." Neurology 49(3): 786-794. 
Jack, C. R., Jr., C. K. Twomey, A. R. Zinsmeister, F. W. Sharbrough, R. C. Petersen and G. D. Cascino (1989). "Anterior temporal lobes and hippocampal formations: normative volumetric measurements from MR images in young adults." Radiology 172(2): 549-554.

Jones, S. E., B. R. Buchbinder and I. Aharon (2000). "Three-dimensional mapping of cortical thickness using Laplace's equation." Hum Brain Mapp 11(1): 12-32.

Kennedy, K. M., K. I. Erickson, K. M. Rodrigue, M. W. Voss, S. J. Colcombe, A. F. Kramer, J. D. Acker and N. Raz (2009). "Age-related differences in regional brain volumes: a comparison of optimized voxel-based morphometry to manual volumetry." Neurobiol Aging 30(10): 1657-1676.

Kramer, A. F., S. J. Colcombe, E. McAuley, P. E. Scalf and K. I. Erickson (2005). "Fitness, aging and neurocognitive function." Neurobiol Aging 26 Suppl 1: 124-127.

Landfield, P. W., E. M. Blalock, K. C. Chen and N. M. Porter (2007). "A new glucocorticoid hypothesis of brain aging: implications for Alzheimer's disease." $\underline{\text { Curr }}$ Alzheimer Res 4(2): 205-212.

Lerch, J. P., J. C. Pruessner, A. Zijdenbos, H. Hampel, S. J. Teipel and A. C. Evans (2005). "Focal decline of cortical thickness in Alzheimer's disease identified by computational neuroanatomy." Cereb Cortex 15(7): 995-1001.

Leritz, E. C., J. Shepel, V. J. Williams, L. A. Lipsitz, R. E. McGlinchey, W. P. Milberg and D. H. Salat (2014). "Associations between T1 white matter lesion volume and regional white matter microstructure in aging." Hum Brain Mapp 35(3): 1085-1100.

Levine, B. D. (2008). ".VO2max: what do we know, and what do we still need to know?" J Physiol 586(1): 25-34.

Lupien, S. J., B. S. McEwen, M. R. Gunnar and C. Heim (2009). "Effects of stress throughout the lifespan on the brain, behaviour and cognition." Nat Rev Neurosci 10(6): 434-445. 
Mu, Q., J. Xie, Z. Wen, Y. Weng and Z. Shuyun (1999). "A quantitative MR study of the hippocampal formation, the amygdala, and the temporal horn of the lateral ventricle in healthy subjects 40 to 90 years of age." AJNR Am J Neuroradiol 20(2): 207-211.

Mueller, E. A., M. M. Moore, D. C. Kerr, G. Sexton, R. M. Camicioli, D. B. Howieson, J. F. Quinn and J. A. Kaye (1998). "Brain volume preserved in healthy elderly through the eleventh decade." Neurology 51(6): 1555-1562.

Ohnishi, T., H. Matsuda, T. Tabira, T. Asada and M. Uno (2001). "Changes in brain morphology in Alzheimer disease and normal aging: is Alzheimer disease an exaggerated aging process?" AJNR Am J Neuroradiol 22(9): 1680-1685.

Podewils, L. J., E. Guallar, L. H. Kuller, L. P. Fried, O. L. Lopez, M. Carlson and C. G. Lyketsos (2005). "Physical activity, APOE genotype, and dementia risk: findings from the Cardiovascular Health Cognition Study." Am J Epidemiol 161(7): 639-651.

Raz, N., P. Ghisletta, K. M. Rodrigue, K. M. Kennedy and U. Lindenberger (2010). "Trajectories of brain aging in middle-aged and older adults: regional and individual differences." Neuroimage 51(2): 501-511.

Raz, N., F. M. Gunning, D. Head, J. H. Dupuis, J. McQuain, S. D. Briggs, W. J. Loken, A. E. Thornton and J. D. Acker (1997). "Selective aging of the human cerebral cortex observed in vivo: differential vulnerability of the prefrontal gray matter." Cereb. Cortex 7(3): 268-282.

Raz, N., U. Lindenberger, K. M. Rodrigue, K. M. Kennedy, D. Head, A. Williamson, C. Dahle, D. Gerstorf and J. D. Acker (2005). "Regional brain changes in aging healthy adults: general trends, individual differences and modifiers." Cereb. Cortex 15(11): 16761689.

Resnick, S. M., A. F. Goldszal, C. Davatzikos, S. Golski, M. A. Kraut, E. J. Metter, R. N. Bryan and A. B. Zonderman (2000). "One-year age changes in MRI brain volumes in older adults." Cereb Cortex 10(5): 464-472. 
Resnick, S. M., D. L. Pham, M. A. Kraut, A. B. Zonderman and C. Davatzikos (2003). "Longitudinal magnetic resonance imaging studies of older adults: a shrinking brain." Neurosci 23(8): 3295-3301.

Reuter, M., N. J. Schmansky, H. D. Rosas and B. Fischl (2012). "Within-subject template estimation for unbiased longitudinal image analysis." Neuroimage 61(4): 1402-1418.

Rovio, S., G. Spulber, L. J. Nieminen, E. Niskanen, B. Winblad, J. Tuomilehto, A. Nissinen, H. Soininen and M. Kivipelto (2010). "The effect of midlife physical activity on structural brain changes in the elderly." Neurobiol Aging 31(11): 1927-1936.

Salat, D. H., R. L. Buckner, A. Z. Snyder, D. N. Greve, R. S. Desikan, E. Busa, J. C. Morris, A. M. Dale and B. Fischl (2004). "Thinning of the cerebral cortex in aging." Cereb. Cortex 14(7): 721-730.

Sapolsky, R. M. (1999). "Glucocorticoids, stress, and their adverse neurological effects: relevance to aging." Exp Gerontol 34(6): 721-732.

Saur, L., P. P. Baptista, P. N. de Senna, M. F. Paim, P. do Nascimento, J. Ilha, P. B. Bagatini, M. Achaval and L. L. Xavier (2014). "Physical exercise increases GFAP expression and induces morphological changes in hippocampal astrocytes." Brain Struct Funct 219(1): 293-302.

Segonne, F., A. M. Dale, E. Busa, M. Glessner, D. Salat, H. K. Hahn and B. Fischl (2004). "A hybrid approach to the skull stripping problem in MRI." Neuroimage 22(3): 1060-1075.

Sled, J. G., A. P. Zijdenbos and A. C. Evans (1998). "A nonparametric method for automatic correction of intensity nonuniformity in MRI data." $\underline{\text { IEEE Trans Med Imaging }}$ 17(1): 87-97.

Smith, P. J. (2012). "Physical activity, vascular health, and cognitive impairment." Arch Intern Med 172(1): 83; author reply 84. 
Thielke, S. and P. Diehr (2012). "Transitions among Health States Using 12 Measures of Successful Aging in Men and Women: Results from the Cardiovascular Health Study." $\mathbf{J}$ Aging Res 2012: 243263.

Thomas, A. G., A. Dennis, P. A. Bandettini and H. Johansen-Berg (2012). "The effects of aerobic activity on brain structure." Front Psychol 3: 86.

Tseng, B. Y., J. Uh, H. C. Rossetti, C. M. Cullum, R. F. Diaz-Arrastia, B. D. Levine, H. Lu and R. Zhang (2013). "Masters athletes exhibit larger regional brain volume and better cognitive performance than sedentary older adults." J Magn Reson Imaging 38(5): 11691176.

Utevsky, A. V., D. V. Smith and S. A. Huettel (2014). "Precuneus is a functional core of the default-mode network." J Neurosci 34(3): 932-940.

van Praag, H., B. R. Christie, T. J. Sejnowski and F. H. Gage (1999). "Running enhances neurogenesis, learning, and long-term potentiation in mice." Proc Natl Acad Sci U S A 96(23): 13427-13431.

Voss, M. W., L. S. Nagamatsu, T. Liu-Ambrose and A. F. Kramer (2011). "Exercise, brain, and cognition across the life span." J Appl Physiol (1985) 111(5): 1505-1513.

Weuve, J., J. H. Kang, J. E. Manson, M. M. Breteler, J. H. Ware and F. Grodstein (2004). "Physical activity, including walking, and cognitive function in older women." JAMA 292(12): 1454-1461. 


\section{Chapter 4}

\section{Cerebral Cortical Thickness Correlates with Autonomic Outflow}

(Submitted to Brain Structure and Function July 20, 2016: BSAF-D-16-00378)

\subsection{Introduction}

Chronic levels of sympathetic and parasympathetic outflow exert important implications for homeostasis and long-term health. Within the context of cardiovascular health, chronic elevations in sympathetic outflow, and/or reductions in parasympathetic cardiac control, contribute to the pathogenesis and progression of atherosclerosis, vascular wall thickening, cardiac damage (Amiya, Watanabe et al. 2014, Chistiakov, Ashwell et al. 2015) and disturbed cardiac rhythms (Pellman and Sheikh 2015), which combine to increase the risk of morbidity and mortality (Schmidt, Muller-Werdan et al. 2005). Advancing age often (Ebert, Morgan et al. 1992), but not always (Greaney, Schwartz et al. 2013), increases baseline muscle sympathetic nerve activity (MSNA). More consistently, advancing age decreases cardiovagal baroreflex sensitivity (Ebert, Morgan et al. 1992, Monahan, Dinenno et al. 2001). Combined, these outcomes suggest a chronic state of heightened peripheral sympathetic tone with parasympathetic withdrawal (Meredith, Eisenhofer et al. 1993, Monahan, Dinenno et al. 2001, Thayer and Lane 2007, Lambert, Dawood et al. 2008, Freeling and Li 2015). The mechanisms affecting this agerelated autonomic dysregulation are not clear. Decreased baroreflex sensitivity accounts for some of the decrease in parasympathetic control of heart rate, as inferred by measures of cardiovagal baroreflex sensitivity (Ebert, Morgan et al. 1992, Monahan, Dinenno et al. 2001). However, age exerts little effect on baroreflex control of MSNA whether levels of sympathetic burst activity are elevated (Ebert, Morgan et al. 1992) or not (Greaney, Schwartz et al. 2013).

Alterations in cerebral cortex structure may contribute to these age-related autonomic changes. Certainly, experimental models in lower animals (Cechetto and Chen 1990, Dampney 1994, Verberne 1996), as well as clinical (Norris, Froggatt et al. 1978, 
Critchley, Mathias et al. 2003, Woo, Macey et al. 2003, Soros and Hachinski 2012, Woo, Yadav et al. 2014), and observational studies in humans (Critchley, Corfield et al. 2000) (Cechetto and Shoemaker 2009, Thayer, Sollers et al. 2009, Shoemaker and Goswami 2015), indicate that several cortical sites modulate autonomic cardiovascular control. These regions include, but are not limited to, the IC, ACC, and MPFC (Barron and Chokroverty 1993, Soufer, Bremner et al. 1998, Critchley, Corfield et al. 2000, Gianaros, Van Der Veen et al. 2004, Gianaros, Derbyshire et al. 2005). Inasmuch as structure forms a basis of function, changes in cortical thickness in these regions may affect the neurologic outcomes.

Cerebral cortex atrophy begins in the third decade of life, with disproportionately high losses in the forebrain regions (Raz, Williamson et al. 2000). Average losses are estimated at roughly $15 \%$ of the cerebral cortex and $25 \%$ of the cerebral white matter between ages 30 and 90 (Jernigan, Archibald et al. 2001). It is noteworthy that those regions associated with autonomic cardiovascular control exist within regions that display high sensitivity towards age-related cortical atrophy. Nonetheless, age-related reductions in cortical tissue show considerable individual variation in their rate, extent, and location (Raz, Gunning et al. 1997, Raz, Rodrigue et al. 2007, Fjell and Walhovd 2010). This inter-individual variation offers an opportunity to explore potential relationships between cortical structure and autonomic changes in aging adults. Specifically, this study tested the hypothesis that inter-individual differences in cortical atrophy predict age-related changes in autonomic outflow. If so, then details related to cortical thickness would form a possible basis for explaining age-related autonomic dysregulation.

\subsection{Methods}

\subsubsection{Participants}

A total of 55 healthy, active individuals participated in this study across a range of fitness and age (26-81mL/kg/min; 21-73 years; 18 female). Table 4.1 provides group characteristics. All participants were right-handed, non-smokers, free of medications, and without diagnosed hypertension, diabetes, vascular or neurological impairments. The University of Western Ontario Health Sciences Ethics Review Board approved this study 
and adhered to the Declaration of Helsinki. Each participant provided informed, written consent.

\subsubsection{Assessment of Cardiorespiratory Fitness}

A graded treadmill exercise test, conducted under standard clinical observation, provided information regarding each subject's peak oxygen uptake $\left(\mathrm{VO}_{2 \max }\right)$. During this test, expired air samples were taken at 3-second intervals until the point of volitional exhaustion. Based on the American College of Sports Medicine guidelines (ACSM 1995), $\mathrm{VO}_{2 \max }$ was determined by meeting the following criteria: (1) $\mathrm{VO}_{2}$ ceased to increase with increasing workloads (plateau); (2) heart rate reached the age-predicted maximum value (220-age); and (3) respiratory exchange ratio was $>1.0$.

\subsubsection{Physiological Data Acquisition}

Participants reported to the laboratory following a $12 \mathrm{~h}$ abstinence from nicotine, alcohol, caffeine and intense physical exertion. Heart rate was measured using a standard threelead electrocardiogram. Continuous tracings of arterial blood pressure through finger photoplethysmography (Finometer; Finapres Medical Systems, Amsterdam, The Netherlands), and calibrated to manual sphygmomanometer blood pressure measurements, enabled calculation of the mathematical mean pressure throughout the protocol. The Modelflow algorithm provided an index of cardiac output from the finger blood pressure tracings (Wesseling, Jansen et al. 1993), incorporating participant's sex, age, height, and weight. A respiratory belt (Pneumotrace II, ADInstruments, Colorado Springs, Colo., USA) was secured around the thorax for collection of respiratory rate. All analog measures were sampled at $1000 \mathrm{~Hz}$ and stored for offline analysis (LabChart7 software; ADInstruments).

\subsubsection{Cardiovagal Baroreflex Sensitivity}

The sequence method, performed on approximately 240 consecutive cardiac cycles at rest, provided a measure of the spontaneous cardiovagal BRS (Blaber, Yamamoto et al. 1995, Parlow, Viale et al. 1995). On the basis of evidence that the R-R interval is generally modulated within the same cardiac cycle (Pickering and Davies 1973), data 
were analyzed using either a lag 0 , lag 1 or a lag 2 approach to achieve the greatest number of sequences (Blaber, Yamamoto et al. 1995). The mean slope of the identified sequences was taken to represent average cardiovagal baroreflex gain.

\subsubsection{Heart Rate Variability}

$\mathrm{R}-\mathrm{R}$ intervals were collected during $10 \mathrm{~min}$ of quiet supine rest. Participants were instructed to remain awake and still.

Time domain HRV analyses included the standard deviation of normal-to-normal R-R intervals (SDNN), while frequency domain analysis included the high-frequency (0.15$0.4 \mathrm{~Hz}$ ) component of the power spectral band (expressed logarithmically, ln-hf), the standard deviation of the width of the Poincaré plot (SD1), and total spectral power.

\subsubsection{Sympathetic Neural Recordings}

Sympathetic neural recordings were obtained in the right peroneal nerve by microneurography, using standard procedures originally defined by Hagbarth (Hagbarth and Vallbo 1968), and reported frequently by our laboratory (Steinback, Salzer et al. 2009, Goswami, Frances et al. 2012) (662C-3; Bioengineering of University of Iowa, Iowa City, Iowa, USA). Integrated sympathetic nerve activity was expressed both as burst frequency (the number of bursts per minute) and burst incidence (the number of bursts per 100 heart beats). Total MSNA was quantified as the product of mean normalized burst amplitude and burst frequency.

\subsubsection{Neuroimaging Data Acquisition}

Participants completed a structural magnetic resonance neuroimaging session (Robarts Research Institute Centre for Functional and Metabolic Imaging) following a $12 \mathrm{~h}$ abstinence from nicotine, alcohol, caffeine and intense physical exertion. All imaging data were collected using a whole body 3-Tesla imaging system (Magnetom Prisma, Siemens Medical Solutions, Erlangen, Germany) with a 32-channel head coil (Barberi, Gati et al. 2000). A high-resolution $T_{1}$-weighted structural volume was acquired with a 3D MPRAGE sequence at the beginning of the scanning session (sagittal, matrix 256x240mm, voxel resolution 1.0x1.0x1.0mm, 1mm slice thickness, no gap, flip angle 
$9^{\circ}, \mathrm{TE}=2.98 \mathrm{~ms}, \mathrm{TI}=900 \mathrm{~ms}, \mathrm{TR}=2.3 \mathrm{~ms}$ ). A head cradle packed with foam padding limited head movement. Each subject received instruction to avoid head movements during the imaging period.

\subsubsection{Neuroimaging Data Analysis}

Cortical thickness analysis was performed using Brain Voyager 2.8.4 (BVQX, Brain Innovation, Maastricht, Netherlands). The Laplace method provided measures of cortical thickness for each subject's right and left hemisphere (Jones, Buchbinder et al. 2000). Spatial intensity inhomogeneities in the original $T_{1}$-weighted scans were corrected and converted into volumes with $1.0 \mathrm{~mm}$ isotropic voxel resolution using sinc-interpolation. These volumes were transformed spatially into standard Talairach space. Once normalized, an automatic segmentation of gray and white-matter boundaries was applied, and images were resampled to $0.5 \mathrm{~mm}$ isovoxel resolution. Topological errors such as "bridges" and remaining fragments of dura mater or cerebellum were corrected manually. Additionally, manual correction of gray and white matter boundaries was performed on a slice-by-slice and individual basis. The reconstructed cortical hemispheres were morphed into a folded three-dimensional mesh. Cortex-based alignment reduced the effect of anatomical variability and improved the spatial correspondence of cortical areas between individual brains while producing a spherical representation of the folded cortex. Finally, curvature information of individual brains was used for inter-subject alignment (Goebel, Esposito et al. 2006), and resulted in average curvature maps for each hemisphere $(n=55)$.

\subsubsection{Statistical Analysis}

To assess the relationship between autonomic function and regional brain tissue thickness, we performed a full cortex correlation analysis in Brain Voyager using each individual's autonomic and cortical thickness variables. Simply stated, a linear regression model assessed the relationship between each participant's BRS, HRV and MSNA values, and the estimated peak cortical thickness value at 42 cortical sites bilaterally. The final correlation maps were adapted by increasing the minimum threshold value $(\mathrm{p}<0.05)$ and adding a cluster threshold $\left(10 \mathrm{~mm}^{2}\right)$. Subsequently, an initial series of linear regressions examined how cortical thickness at the predefined regions of interest 
predicted the autonomic variables. Significant associations were followed by multiple linear regression to assess independence of the relationships after adjusting for age and cardiorespiratory fitness $\left(\mathrm{VO}_{2 \max }\right)$. A threshold for significance was set at $\mathrm{p}<0.05$. All regression analyses passed the normality test, as assessed by the Shapiro-Wilk test for normal distribution.

\subsection{Results}

\subsubsection{Whole-Brain Regression Results}

Significant positive (BRS, HRV: SD1, SDNN, ln-hf, total spectral power) and negative (muscle sympathetic nerve activity: burst frequency, burst incidence, total MSNA) correlations were widespread and bilateral when whole-brain cortical thickness was regressed with values of autonomic outflow. These peak-vertex correlation results are given in Appendix 1 for each variable across all 42 cortical sites. Selected whole-brain results including baroreflex sensitivity, ln-high frequency, and MSNA burst frequency, are illustrated in Figure 4.1.

Table 4.2 illustrates significant relationships from the preliminary whole-brain linear regression analysis. BRS correlated with the average right hemisphere cortical thickness $(\mathrm{p}<0.05)$, and the left insula $(\mathrm{p}=0.04)$. Cortical thickness was correlated with SDNN over the right hemisphere $(\mathrm{p}=0.03)$, as well as the right $(\mathrm{p}=0.01)$ and left MPFC $(\mathrm{p}<0.001)$. Total spectral power (HRV) had a significant relationship with cortical thickness over the right hemisphere $(\mathrm{p}=0.02)$, as well as the right $(\mathrm{p}=0.02)$ and left MPFC $(\mathrm{p}<0.001)$. SD1 was correlated with cortical thickness at the left MPFC ( $\mathrm{p}=0.01$ ). Burst frequency, burst incidence, total MSNA, and ln-high frequency of HRV were correlated with the average cortical thickness in the right and left hemisphere, as well as the regions of interest, namely the bilateral MPFC and bilateral insula.

Subsequent multiple regression analysis of significant associations from the linear regression analysis (Table 4.3) included age and fitness as possible covariates. Fitness $\left(\mathrm{VO}_{2 \max }\right)$ did not alter the relationship between cortical thickness and any of our autonomic variables, with the exception of SD1 $(\mathrm{p}<0.05)$. Age influenced the relationship between cortical thickness and HRV (total power ( $\mathrm{p}=0.03$ ), ln-high frequency power 
$(\mathrm{p}=0.02), \mathrm{SD} 1(\mathrm{p}<0.005))$, as well as burst incidence $(\mathrm{p}=0.02)$. The thickness of the left MPFC was a dominant predictor of SDNN ( $\mathrm{p}=0.01)$, HRV total power $(\mathrm{p}=0.01)$, ln-high frequency power $(\mathrm{p}=0.05)$, burst frequency $(\mathrm{p}=0.01)$, burst incidence $(\mathrm{p}=0.02)$, and total MSNA ( $\mathrm{p}=0.05$ ). Figure 4.2 illustrates the consistent presence of the left MPFC in these regressions. In addition, burst frequency and burst incidence can be predicted by the average cortical thickness of the right hemisphere ( $\mathrm{p}=0.03$ and 0.02 , respectively), while burst incidence had further significant relationships with the average cortical thickness of the left hemisphere $(\mathrm{p}=0.01)$ and left insula $(\mathrm{p}=0.05)$. 
Table 4.1 Anthropometric and baseline autonomic data (mean \pm SD).

\begin{tabular}{rcc}
\hline & Average Group Data & Value Range \\
Age (years) & $45 \pm 15$ & $21-73$ \\
Resting MAP (mmHg) & $87 \pm 9$ & $69-107$ \\
Resting Heart Rate $(\mathrm{bpm})$ & $57 \pm 9$ & $41-90$ \\
$\mathrm{BMI}\left(\mathrm{kg} / \mathrm{m}^{2}\right)$ & $24 \pm 3$ & $17-36$ \\
$\mathrm{VO}_{2 \mathrm{max}}(\mathrm{mL} / \mathrm{kg} / \mathrm{min})$ & $51 \pm 12$ & $26-81$ \\
Cardiac Output $(\mathrm{L} / \mathrm{min})$ & $2.8 \pm 0.7$ & $1-4$ \\
SDNN $(\mathrm{ms})$ & $23 \pm 17$ & $2-84$ \\
Total Power $\left(\mathrm{ms}^{2}\right)$ & $3743 \pm 3306$ & $14-116$ \\
$\operatorname{lnHF}\left(\mathrm{ln} \mathrm{ms} \mathrm{s}^{2}\right)$ & $6.1 \pm 1.5$ & $2-12206$ \\
$\mathrm{SD} 1(\mathrm{~ms})$ & $28 \pm 27$ & $4-112$ \\
Baroreflex Sensitivity (ms/mmHg) & $28 \pm 11$ & $9-50$ \\
Burst Frequency (bursts/min) & $48 \pm 19$ & $14-83$ \\
Burst Incidence (bursts/100 heart beats) & $5 \pm 3$ & $1-15$ \\
\hline Total MSNA (V/min) & &
\end{tabular}

MAP, mean arterial pressure; $\mathrm{BMI}$, body mass index; $\mathrm{VO}_{2 \max }$, maximal oxygen consumption; SDNN, standard deviation of normal to normal intervals; HF, high frequency-power; SD1, Poincare plot width; MSNA, muscle sympathetic nerve activity. Burst frequency, burst incidence and total MSNA: $n=25$. Cardiac output indexed to body surface area. 
Table 4.2 Linear regression of autonomic variables on cortical thickness.

\begin{tabular}{lccccccc} 
& & RH & LH & RH & LH & R Insula & L Insula \\
BRS (ms/mmHg) & $\mathrm{P}$ & $\mathbf{0 . 0 4 6}$ & 0.120 & 0.095 & 0.054 & 0.088 & $\mathbf{0 . 0 4 1}$ \\
& $\mathrm{R}$ & 0.270 & 0.212 & 0.227 & 0.261 & 0.232 & 0.276 \\
Heart Rate Variability & & & & & & & \\
SDNN (ms) & $\mathrm{P}$ & $\mathbf{0 . 0 3 2}$ & 0.142 & $\mathbf{0 . 0 1 4}$ & $<\mathbf{0 . 0 0 1}$ & 0.194 & 0.100 \\
& $\mathrm{R}$ & 0.295 & 0.204 & 0.336 & 0.467 & 0.181 & 0.229 \\
Total Power (ms ${ }^{2}$ ) & $\mathrm{P}$ & $\mathbf{0 . 0 1 5}$ & 0.100 & $\mathbf{0 . 0 1 5}$ & $<\mathbf{0 . 0 0 1}$ & 0.125 & 0.070 \\
& $\mathrm{R}$ & 0.333 & 0.229 & 0.331 & 0.486 & 0.213 & 0.251 \\
lnHF (ln ms ${ }^{2}$ ) & $\mathrm{P}$ & $\mathbf{0 . 0 0 9}$ & $\mathbf{0 . 0 3 8}$ & $\mathbf{0 . 0 0 9}$ & $\mathbf{0 . 0 0 3}$ & $\mathbf{0 . 0 1 1}$ & $\mathbf{0 . 0 0 6}$ \\
& $\mathrm{R}$ & 0.355 & 0.286 & 0.357 & 0.400 & 0.347 & 0.370 \\
SD1 (ms) & $\mathrm{P}$ & 0.082 & 0.579 & 0.051 & $\mathbf{0 . 0 0 9}$ & 0.187 & $0.335^{\dagger}$ \\
& $\mathrm{R}$ & 0.241 & 0.078 & 0.270 & 0.354 & 0.184 & 0.135 \\
Sympathetic Nerve Activity & & & & & & & \\
BF (bursts/min) & & & & & & & \\
& $\mathrm{P}$ & $<\mathbf{0 . 0 0 1}$ & $<\mathbf{0 . 0 0 1}$ & $<\mathbf{0 . 0 0 1}$ & $<\mathbf{0 . 0 0 1}$ & $<\mathbf{0 . 0 0 1}$ & $<\mathbf{0 . 0 0 1}$ \\
BI (bursts/100 heart beats) & $\mathrm{R}$ & 0.742 & 0.731 & 0.664 & 0.809 & 0.708 & 0.717 \\
& $\mathrm{P}$ & $<\mathbf{0 . 0 0 1}$ & $<\mathbf{0 . 0 0 1}$ & $<\mathbf{0 . 0 0 1}$ & $<\mathbf{0 . 0 0 1}$ & $<\mathbf{0 . 0 0 1}$ & $<\mathbf{0 . 0 0 1}$ \\
Total MSNA (V/min) & $\mathrm{R}$ & 0.768 & 0.779 & 0.670 & 0.784 & 0.738 & 0.742 \\
& $\mathrm{P}$ & $<\mathbf{0 . 0 0 1}$ & $<\mathbf{0 . 0 0 1}$ & $<\mathbf{0 . 0 0 1}$ & $<\mathbf{0 . 0 0 1}$ & $\mathbf{0 . 0 0 2}$ & $\mathbf{0 . 0 0 2}$ \\
& $\mathrm{R}$ & 0.648 & 0.632 & 0.613 & 0.750 & 0.593 & 0.598 \\
\hline
\end{tabular}

$\mathrm{P}$ value and correlation coefficient $(\mathrm{R})$ determined from regression values. $\mathrm{RH}$, right hemisphere; LH, left hemisphere; MPFC, medial prefrontal cortex; BRS, baroreflex sensitivity; SDNN, standard deviation of normal to normal intervals; HF, high frequencypower; SD1, Poincare plot width; BF, burst frequency; BI, burst incidence; MSNA, muscle sympathetic nerve activity. Bold type indicates significant relationships $(p<0.05)$ carried into subsequent multiple regression. 
Table 4.3 Results of multiple linear regression based on simple linear regression.

\begin{tabular}{|c|c|c|c|c|}
\hline Dependent Variable & Independent Variable & Std. Coefficient & Std. Error & $P$ value \\
\hline \multirow{4}{*}{ Baroreflex Sensitivity } & Age & -0.301 & 0.194 & 0.127 \\
\hline & $\mathrm{VO}_{2 \max }$ & -0.012 & 0.227 & 0.957 \\
\hline & Right Hemisphere & 4.524 & 10.976 & 0.682 \\
\hline & L Insula & 3.889 & 6.081 & 0.525 \\
\hline \multicolumn{5}{|l|}{ Heart Rate Variability } \\
\hline \multirow[t]{5}{*}{ SDNN } & Age & -0.354 & 0.286 & 0.221 \\
\hline & $\mathrm{VO}_{2 \max }$ & -0.315 & 0.329 & 0.343 \\
\hline & Right Hemisphere & 11.568 & 17.655 & 0.515 \\
\hline & R MPFC & 6.425 & 6.009 & 0.290 \\
\hline & L MPFC & 20.211 & 7.940 & 0.014 \\
\hline \multirow[t]{5}{*}{ Total Power } & Age & -80.752 & 36.362 & 0.031 \\
\hline & $\mathrm{VO}_{2 \max }$ & -52.573 & 39.320 & 0.188 \\
\hline & Right Hemisphere & 1946.183 & 2117.005 & 0.363 \\
\hline & R MPFC & 697.027 & 725.304 & 0.341 \\
\hline & L MPFC & 2585.336 & 947.881 & 0.009 \\
\hline \multirow[t]{8}{*}{$\operatorname{lnHF}$} & Age & -0.0422 & 0.0176 & 0.021 \\
\hline & $\mathrm{VO}_{2 \max }$ & -0.0225 & 0.0183 & 0.226 \\
\hline & Right Hemisphere & 1.013 & 0.954 & 0.294 \\
\hline & Left Hemisphere & 0.345 & 0.902 & 0.704 \\
\hline & R MPFC & 0.527 & 0.322 & 0.108 \\
\hline & L MPFC & 0.895 & 0.442 & 0.049 \\
\hline & R Insula & 0.651 & 0.648 & 0.320 \\
\hline & L Insula & 0.712 & 0.530 & 0.185 \\
\hline \multirow[t]{3}{*}{ SD1 } & Age & -0.831 & 0.274 & 0.004 \\
\hline & $\mathrm{VO}_{2 \max }$ & -0.670 & 0.315 & 0.039 \\
\hline & L MPFC & 11.904 & 7.602 & 0.124 \\
\hline \multicolumn{5}{|l|}{ Sympathetic Nerve Activity } \\
\hline \multirow[t]{8}{*}{ Burst Frequency } & Age & 0.094 & 0.196 & 0.638 \\
\hline & $\mathrm{VO}_{2 \max }$ & -0.216 & 0.228 & 0.355 \\
\hline & Right Hemisphere & -29.940 & 12.893 & 0.031 \\
\hline & Left Hemisphere & -20.022 & 11.586 & 0.099 \\
\hline & R MPFC & -2.611 & 2.812 & 0.364 \\
\hline & L MPFC & -10.844 & 3.903 & 0.012 \\
\hline & R Insula & -11.708 & 7.259 & 0.122 \\
\hline & L Insula & -10.229 & 5.454 & 0.075 \\
\hline \multirow[t]{8}{*}{ Burst Incidence } & Age & 0.710 & 0.272 & 0.017 \\
\hline & $\mathrm{VO}_{2 \max }$ & 0.321 & 0.415 & 0.448 \\
\hline & Right Hemisphere & -56.569 & 21.581 & 0.016 \\
\hline & Left Hemisphere & -51.204 & 18.065 & 0.010 \\
\hline & R MPFC & -4.769 & 4.829 & 0.335 \\
\hline & L MPFC & -16.960 & 6.943 & 0.024 \\
\hline & R Insula & -20.574 & 12.465 & 0.114 \\
\hline & L Insula & -19.645 & 9.188 & 0.045 \\
\hline \multirow[t]{6}{*}{ Total MSNA } & Age & 8.385 & 9.074 & 0.366 \\
\hline & $\mathrm{VO}_{2 \max }$ & -8.750 & 13.682 & 0.530 \\
\hline & Right Hemisphere & -946.417 & 868.848 & 0.289 \\
\hline & Left Hemisphere & -513.840 & 755.908 & 0.504 \\
\hline & L MPFC & -541.884 & 255.557 & 0.047 \\
\hline & L Insula & -219.490 & 360.751 & 0.550 \\
\hline
\end{tabular}

$\mathrm{R}$, right hemisphere; L, left hemisphere; MPFC, medial prefrontal cortex; SDNN, standard deviation of normal to normal intervals; HF, high frequency-power; SD1, Poincare plot width; MSNA, muscle sympathetic nerve activity. Bold type indicates significant relationships $(\mathrm{p}<0.05)$. 
Figure 4.1 Whole-brain correlation of selected autonomic variables and cortical thickness.

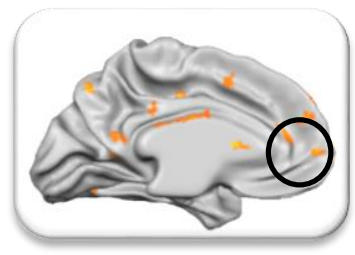

Baroreflex Sensitivity
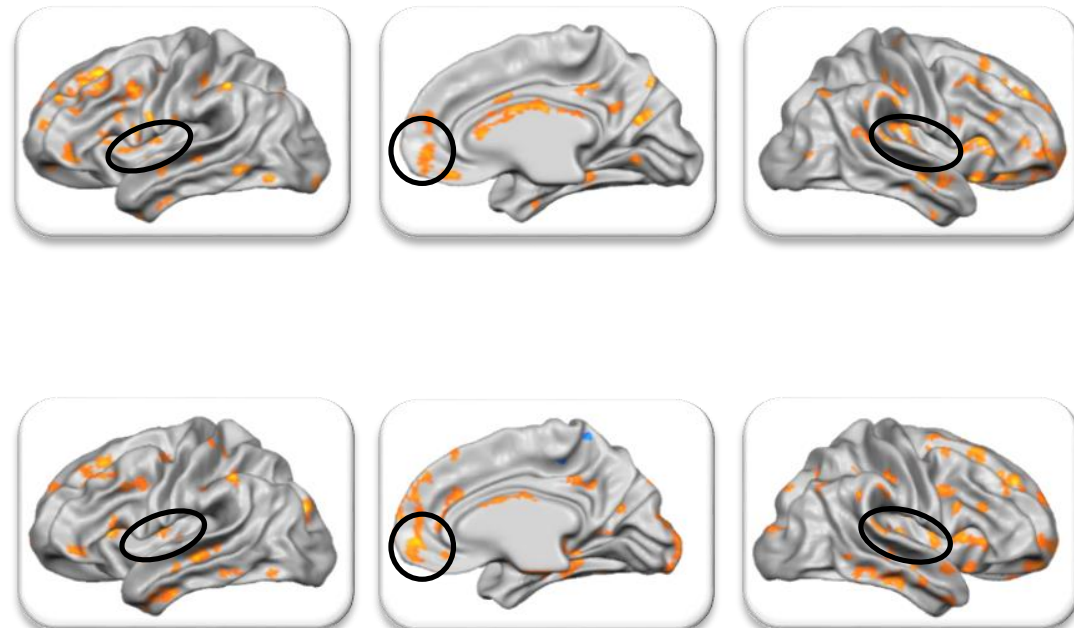

In-High Frequency
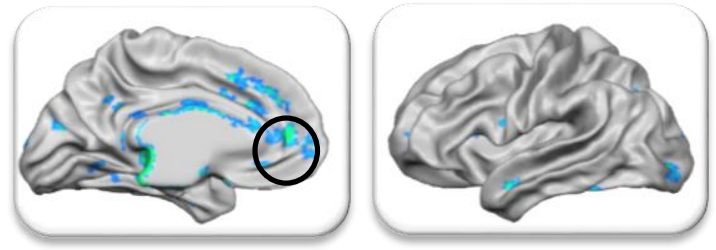

Burst Frequency

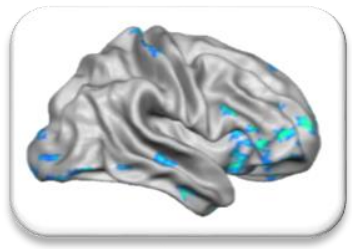

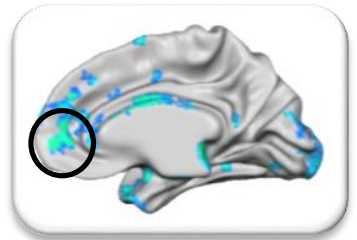
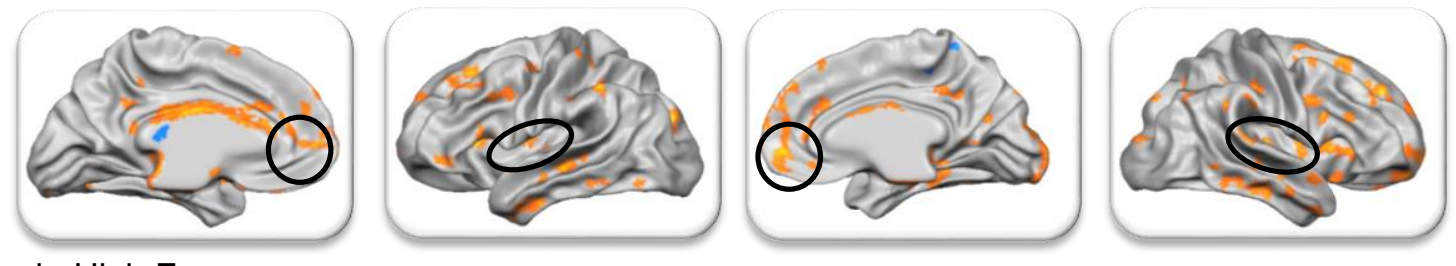

$p<0.026062$
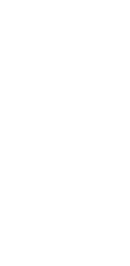

Top row: baroreflex sensitivity; middle row: ln high frequency (heart rate variability); bottom row: burst frequency (muscle sympathetic nerve activity). Pictures at right: right hemisphere; pictures at left: left hemisphere. Highlighted circles denote medial prefrontal and insula cortex regions of interest. Results are shown at $\mathrm{p}<0.05$, corrected by cluster size $\left(10 \mathrm{~mm}^{2}\right)$. Color scale at right denotes t values. 
Figure 4.2 Regression analysis of significant autonomic variables with cortical thickness at the left medial prefrontal cortex.
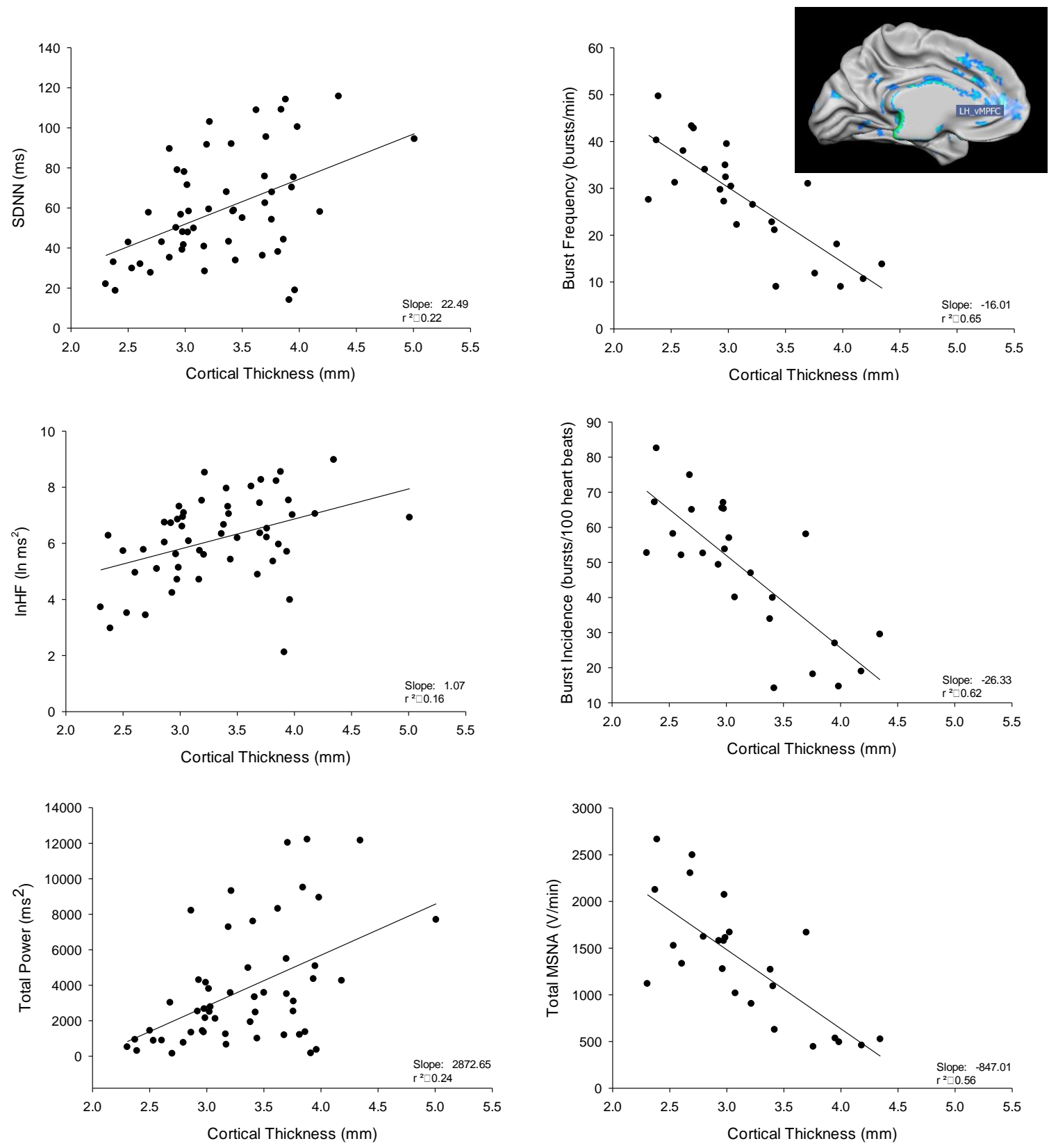

Talairach coordinates of peak vertex at region of interest (inset): $-12,42,12$. SDNN, standard deviation of normal to normal intervals; HF, high frequency power; MSNA, muscle sympathetic nerve activity. Sympathetic outflow (burst frequency, burst incidence, total MSNA) and cardiovagal indices (SDNN, lnHF, total power) can be predicted by thickness at the left medial prefrontal cortex $(\mathrm{p}<0.05$, Table 4.3$)$. 


\subsection{Discussion}

The current study supports the hypothesis that cortical thickness predicts chronic levels of HRV and MSNA, particularly in the medial prefrontal cortex. Importantly, age was the primary determinant of the variations. The strength of the relationship between our autonomic variables and cortical thickness was not altered following adjustments for cardiorespiratory fitness.

The observation that the thickness of the MPFC predicts chronic levels of high HRV and low MSNA, forms a unique supportive concept towards understanding age-related autonomic dysregulation. However, the lack of relationship between cardiovagal baroreflex sensitivity and the MPFC was unexpected. Specifically, experimental studies in rodents indicate that this region exerts powerful modulatory effects over baroreflex function and has strong reciprocal connections with structures involved in autonomic function including the amygdala, hypothalamus, periaqueductal gray, the nucleus of the tractus solitarus and the caudal and rostral ventrolateral medulla (Neafsey 1990, Chiba and Semba 1991, Hurley, Herbert et al. 1991, Vertes 2004). Further, recent studies conducted in rodents and humans have revealed depressor sites within the ventral region of the MPFC that exhibit heightened levels of activity during periods of behavioral relaxation, including sleep and rest (Critchley, Mathias et al. 2001, Cechetto and Shoemaker 2009). The inverse relationship between MPFC activation and cardiovascular arousal in human neuroimaging studies suggests a direct effect of this region on cardiovascular function (Critchley, Corfield et al. 2000, Wong, Masse et al. 2007, Thayer, Sollers et al. 2009). Nevertheless, the current results do not suggest that cortical thickness at the MPFC is predictive of baroreflex function. One possible explanation for this is that the above results are based on functional associations between MPFC activation patterns and concurrent changes in heart rate, while the cardiovagal baroreflex is a complex signal involving both heart rate and arterial pressure. Other cortical areas such as the dorsal lateral prefrontal cortex may also modulate the baroreceptor reflex arc, since retrograde and anterograde transport studies indicate that this site may provide a quantitatively larger input to the nucleus of the solitary tract than the MPFC (van der Kooy, McGinty et al. 1982, van der Kooy, Koda et al. 1984). Indeed, the current results 
support this connection as the dorsal lateral prefrontal cortex was correlated with baroreflex sensitivity in both the right and left hemispheres (see Appendix 1: superior frontal gyrus).

The current analysis examined the potential impact of cardiorespiratory fitness as a covariate in the age-cortical thickness relationship, and included the conceptual potential for underlying factors related to exercise training and increased fitness. Several factors contribute to this possibility. For example, cardiorespiratory fitness has been linked to improved hippocampal (Erickson, Voss et al. 2011, Varma, Chuang et al. 2014), MPFC (Colcombe, Erickson et al. 2003, Colcombe, Erickson et al. 2006, Kramer, Erickson et al. 2006), and anterior insula volumes (Peters, Dauvermann et al. 2009). In addition, high cardiorespiratory fitness has been associated with less structural brain atrophy (Colcombe, Erickson et al. 2003, Kramer, Erickson et al. 2006, Erickson, Voss et al. 2011) and higher estimates of white matter integrity (Marks, Katz et al. 2011, Johnson, Kim et al. 2012, Voss, Heo et al. 2013, Burzynska, Chaddock-Heyman et al. 2014). Recently, we reported preliminary results indicating a strong association between cardiorespiratory fitness and cortical "reserve" of gray matter at the MPFC (Norton, Heinecke et al. 2015). Furthermore, voxel-based morphometry measures indicate increased prefrontal cortex volume in cardiac patients undergoing cardiac rehabilitation exercise (Anazodo, Shoemaker et al. 2013). Nevertheless, the current results indicate that the strong relationships between cortical thickness and HRV and MSNA variables are not affected by inclusion of $\mathrm{VO}_{2 \max }$ in the regression model. This observation corresponds with the growing understanding that whereas exercise training can minimize the impact of age on autonomic function, the amount of exercise training or improved fitness needed to completely reverse age-related cardiovagal BRS in healthy humans are not known. Also, exercise training can reduce MSNA burst frequency in individuals with clinically or excessively elevated levels, such as those that occur with advanced age or pathology (e.g. hypertension) (Carter and Ray 2015). The current data support a speculation that the impact of exercise training on neural control of the circulation may include improvements to cortical thickness in pertinent brain regions. We acknowledge, however, that contributors to brain health extend well beyond exercise and can include sleep, diet, 
lifestyle, social environment, cognitive stimulation, and genetics, among others. The relative impact of these contributors remains to be reported.

The insula cortex (IC) exhibits strong associations with cardiovascular control in functional imaging studies with humans (Cechetto and Saper 1990, Critchley, Corfield et al. 2000, Kimmerly, O'Leary et al. 2005, Cechetto and Shoemaker 2009, Macey, Wu et al. 2012), clinical studies (Critchley, Mathias et al. 2003, Woo, Macey et al. 2003, Soros and Hachinski 2012, Woo, Yadav et al. 2014), and experimental rodent models (Cechetto and Chen 1990, Verberne and Owens 1998). These associations are representative of strong sensory, as well as efferent top-down, sensitivities of this region. The topographical sensory organization of the insular cortex includes representation of baroreceptor afferent projections into the forebrain (Cechetto and Saper 1987). The relationship between cortical thickness in the insula and its sensitivity to sensory inputs was not studied in the current study. Indeed, the current autonomic indices are baseline values in healthy individuals with a rather narrow range of normal blood pressure values. Therefore, we do not think the current associations between insula cortical thickness and autonomic variables reflect a sensory mechanism. Rather, our approach was to assess the insula-autonomic outflow relationships. In this context, observations that direct stimulation of insular cortex regions produces cardiac damage through a sympathetic mechanism (Oppenheimer, Wilson et al. 1991) point to the potency of this region over autonomic function. The involvement of the IC has also been reported in situations of physical stress such as baroreceptor unloading (Kimmerly, O'Leary et al. 2005), and isometric exercise (Wong, Kimmerly et al. 2007, Goswami, Frances et al. 2012). Moreover, increased activity within the IC occurs during a variety of cognitive maneuvers that elevate autonomic arousal such as gambling (Critchley, Mathias et al. 2001), Stroop task (Gianaros, Van Der Veen et al. 2004), mental arithmetic (Critchley, Corfield et al. 2000) and many more. In the current study, MSNA burst incidence correlated with cortical thickness at the left insula. Thus, the structural approach in this study further supports the IC as an important region in generalized autonomic control. This study was not able to examine the gyri-specific associations across the various gyri in the IC, although such outcomes are apparent in functional MRI studies (King, Menon et al. 1999, Macey, Wu et al. 2012). 
In addition to the regions of interest discussed above, our whole-brain approach exposed correlations between markers of autonomic outflow and cortical thickness of several other prefrontal brain regions - particularly the cingulate gyrus, and the superior frontal gyrus, or dorsal lateral prefrontal cortex. These regions are marked by their associations with functional autonomic outcomes. For example, peripheral changes in blood pressure are reflected in activity within the anterior cingulate cortex, a region that also may integrate peripheral cardiovascular changes with cognitive effort, motor and emotional states (Critchley, Corfield et al. 2000, Matthews, Paulus et al. 2004, Gianaros, Derbyshire et al. 2005). Woodward et al. (2008), and later Winkelmann et al (2016), reported associations between vagally-mediated heart rate variability and activation changes within the anterior mid-cingulate, or rostral dorsal anterior cingulate. In addition, functional magnetic resonance imaging methods exposed increased activity in the dorsal lateral prefrontal cortex during a maximal inspiratory apnea, a known maneuver of sympathoexcitation (Macefield, Gandevia et al. 2006).

\subsubsection{Perspectives}

The balance between sympathetic and parasympathetic regulation of the cardiovascular system is crucial to long-term health and to acute adjustments to stress. Chronic disturbances can contribute to the pathogenesis and progression of several cardiovascular disease states, thereby increasing the risk of morbidity and mortality (Schmidt, MullerWerdan et al. 2005). Our study identifies a set of brain regions whose cortical thickness relates strongly to peripheral autonomic indices involved in cardiovascular control. These regions, specifically the MPFC and IC, along with the superior frontal gyrus, are highly susceptible to age-related cortical atrophy, as well as other pathologies. Moreover, these brain regions are also involved in cognitive and behavioural functions that also tend to decline in advancing age. Our findings are the first to report human data which suggest a link between cortical structure and autonomic function in these sites. Thus, strategies to sustain cortical thickness in these regions are expected to provide effective restraint of autonomic changes that occur with age and, thereby, improve overall health and mobility. 


\subsection{Limitations}

The findings in the current study were limited to healthy participants to avoid comorbidity concerns associated with disease. Given the generalized sympathetic activation in various disorders such as cardiac disease, arthritis and neuropathologies (eg. Parkinson's Disease), it may be that the findings observed in this study are augmented in the disease state. Our region of interest analysis was based on previous literature and experience, both from our laboratory (Kimmerly, O'Leary et al. 2005, Wong, Masse et al. 2007, Cechetto and Shoemaker 2009, Goswami, Frances et al. 2012, Anazodo, Shoemaker et al. 2013, Norton, Luchyshyn et al. 2013) and that of others (Colcombe, Erickson et al. 2006, Macefield, Gandevia et al. 2006, Thayer and Lane 2007, Erickson, Voss et al. 2011). However, as suggested in the Appendices, there are many brain regions that appear to be correlated to autonomic function and may be studied in this context. For example, the dorsal lateral prefrontal cortex, the anterior cingulate cortex and the hippocampus are known to be associated with cardiovascular control (Mulder, Arts et al. 1997, Westerhaus and Loewy 2001, Castle, Comoli et al. 2005, Winkelmann, Thayer et al. 2016) and are affected by age (Raz, Gunning et al. 1997, Good, Johnsrude et al. 2001, Jernigan, Archibald et al. 2001, Allen, Bruss et al. 2005, Colcombe, Erickson et al. 2006, Thayer, Sollers et al. 2009, Anazodo, Shoemaker et al. 2013, Freeling and Li 2015).

\subsection{Conclusion}

The findings in the current study were limited to healthy participants to avoid comorbidity concerns associated with disease. Given the generalized sympathetic activation in various disorders such as cardiac disease, arthritis and neuropathologies (eg. Parkinson's Disease), it may be that the findings observed in this study are augmented in the disease state. Our region of interest analysis was based on previous literature and experience, both from our laboratory (Kimmerly, O'Leary et al. 2005, Wong, Masse et al. 2007, Cechetto and Shoemaker 2009, Goswami, Frances et al. 2012, Anazodo, Shoemaker et al. 2013, Norton, Luchyshyn et al. 2013) and that of others (Colcombe, Erickson et al. 2006, Macefield, Gandevia et al. 2006, Thayer and Lane 2007, Erickson, Voss et al. 2011). However, as suggested in the Appendices, there are many brain regions that appear to be correlated to autonomic function and may be studied in this context. For 
example, the dorsal lateral prefrontal cortex, the anterior cingulate cortex and the hippocampus are known to be associated with cardiovascular control (Mulder, Arts et al. 1997, Westerhaus and Loewy 2001, Castle, Comoli et al. 2005, Winkelmann, Thayer et al. 2016) and are affected by age (Raz, Gunning et al. 1997, Good, Johnsrude et al. 2001, Jernigan, Archibald et al. 2001, Allen, Bruss et al. 2005, Colcombe, Erickson et al. 2006, Thayer, Sollers et al. 2009, Anazodo, Shoemaker et al. 2013, Freeling and Li 2015). 


\subsection{References}

ACSM (1995). Guidelines for Exercise Testing and Prescription. L. W. a. Wilkins. Philadelphia: 160.

Allen, J. S., J. Bruss, C. K. Brown and H. Damasio (2005). "Normal neuroanatomical variation due to age: the major lobes and a parcellation of the temporal region." Neurobiol. Aging 26(9): 1245-1260.

Amiya, E., M. Watanabe and I. Komuro (2014). "The Relationship between Vascular Function and the Autonomic Nervous System." Ann Vasc Dis 7(2): 109-119.

Anazodo, U. C., J. K. Shoemaker, N. Suskin and K. S. St Lawrence (2013). "An investigation of changes in regional gray matter volume in cardiovascular disease patients, pre and post cardiovascular rehabilitation." Neuroimage. Clin 3: 388-395.

Barberi, E. A., J. S. Gati, B. K. Rutt and R. S. Menon (2000). "A transmit-only/receiveonly (TORO) RF system for high-field MRI/MRS applications." Magn Reson. Med 43(2): 284-289.

Barron, K. D. and S. Chokroverty (1993). Anatomy of the autonomic nervous system: brain and brainstem. Clinical Autonomic Disorders, Evaluation and Management. L. PA, Little, Brown and Co: 3-15.

Blaber, A. P., Y. Yamamoto and R. L. Hughson (1995). "Methodology of spontaneous baroreflex relationship assessed by surrogate data analysis." Am J Physiol 268(4 Pt 2): H1682-1687.

Burzynska, A. Z., L. Chaddock-Heyman, M. W. Voss, C. N. Wong, N. P. Gothe, E. A. Olson, A. Knecht, A. Lewis, J. M. Monti, G. E. Cooke, T. R. Wojcicki, J. Fanning, H. D. Chung, E. Awick, E. McAuley and A. F. Kramer (2014). "Physical activity and cardiorespiratory fitness are beneficial for white matter in low-fit older adults." PLoS One 9(9): e107413. 
Carter, J. R. and C. A. Ray (2015). "Sympathetic neural adaptations to exercise training in humans." Auton Neurosci 188: 36-43.

Castle, M., E. Comoli and A. D. Loewy (2005). "Autonomic brainstem nuclei are linked to the hippocampus." Neuroscience 134(2): 657-669.

Cechetto, D. F. and S. J. Chen (1990). "Subcortical sites mediating sympathetic responses from insular cortex in rats." Am. J. Physiol 258(1 Pt 2): R245-R255.

Cechetto, D. F. and C. B. Saper (1987). "Evidence for a viscerotopic sensory representation in the cortex and thalamus in the rat." J Comp Neurol 262(1): 27-45.

Cechetto, D. F. and C. B. Saper (1990). Role of the cerebral cortex in autonomic function, Oxford University Press, New York.

Cechetto, D. F. and J. K. Shoemaker (2009). "Functional neuroanatomy of autonomic regulation." Neuroimage 47(3): 795-803.

Chiba, T. and R. Semba (1991). "Immuno-electronmicroscopic studies on the gammaaminobutyric acid and glycine receptor in the intermediolateral nucleus of the thoracic spinal cord of rats and guinea pigs." J Auton Nerv Syst 36(3): 173-181.

Chistiakov, D. A., K. W. Ashwell, A. N. Orekhov and Y. V. Bobryshev (2015). "Innervation of the arterial wall and its modification in atherosclerosis." $\underline{\text { Auton Neurosci }}$ 193: $7-11$.

Colcombe, S. J., K. I. Erickson, N. Raz, A. G. Webb, N. J. Cohen, E. McAuley and A. F. Kramer (2003). "Aerobic fitness reduces brain tissue loss in aging humans." J Gerontol A Biol Sci Med Sci 58(2): 176-180.

Colcombe, S. J., K. I. Erickson, P. E. Scalf, J. S. Kim, R. Prakash, E. McAuley, S. Elavsky, D. X. Marquez, L. Hu and A. F. Kramer (2006). "Aerobic exercise training increases brain volume in aging humans." J Gerontol A Biol Sci Med Sci 61(11): 11661170. 
Critchley, H. D., D. R. Corfield, M. P. Chandler, C. J. Mathias and R. J. Dolan (2000). "Cerebral correlates of autonomic cardiovascular arousal: a functional neuroimaging investigation in humans." J. Physiol 523 Pt 1: 259-270.

Critchley, H. D., C. J. Mathias and R. J. Dolan (2001). "Neural activity in the human brain relating to uncertainty and arousal during anticipation." Neuron 29(2): 537-545.

Critchley, H. D., C. J. Mathias, O. Josephs, J. O'Doherty, S. Zanini, B. K. Dewar, L. Cipolotti, T. Shallice and R. J. Dolan (2003). "Human cingulate cortex and autonomic control: converging neuroimaging and clinical evidence." Brain 126(Pt 10): 2139-2152.

Dampney, R. A. (1994). "Functional organization of central pathways regulating the cardiovascular system." Physiol Rev 74(2): 323-364.

Ebert, T. J., B. J. Morgan, J. A. Barney, T. Denahan and J. J. Smith (1992). "Effects of aging on baroreflex regulation of sympathetic activity in humans." Am J Physiol $263(3 \mathrm{Pt}$ 2): H798-803.

Erickson, K. I., M. W. Voss, R. S. Prakash, C. Basak, A. Szabo, L. Chaddock, J. S. Kim, S. Heo, H. Alves, S. M. White, T. R. Wojcicki, E. Mailey, V. J. Vieira, S. A. Martin, B. D. Pence, J. A. Woods, E. McAuley and A. F. Kramer (2011). "Exercise training increases size of hippocampus and improves memory." Proc Natl Acad Sci U S A 108(7): 3017-3022.

Fjell, A. M. and K. B. Walhovd (2010). "Structural brain changes in aging: courses, causes and cognitive consequences." Rev Neurosci 21(3): 187-221.

Freeling, J. L. and Y. Li (2015). "Age-related attenuation of parasympathetic control of the heart in mice." Int J Physiol Pathophysiol Pharmacol 7(3): 126-135.

Gianaros, P. J., S. W. Derbyshire, J. C. May, G. J. Siegle, M. A. Gamalo and J. R. Jennings (2005). "Anterior cingulate activity correlates with blood pressure during stress." Psychophysiology 42(6): 627-635. 
Gianaros, P. J., F. M. Van Der Veen and J. R. Jennings (2004). "Regional cerebral blood flow correlates with heart period and high-frequency heart period variability during working-memory tasks: Implications for the cortical and subcortical regulation of cardiac autonomic activity." Psychophysiology 41(4): 521-530.

Goebel, R., F. Esposito and E. Formisano (2006). "Analysis of functional image analysis contest (FIAC) data with brainvoyager QX: From single-subject to cortically aligned group general linear model analysis and self-organizing group independent component analysis." Hum. Brain Mapp 27(5): 392-401.

Good, C. D., I. S. Johnsrude, J. Ashburner, R. N. Henson, K. J. Friston and R. S. Frackowiak (2001). "A voxel-based morphometric study of ageing in 465 normal adult human brains." Neuroimage 14(1 Pt 1): 21-36.

Goswami, R., M. F. Frances, C. D. Steinback and J. K. Shoemaker (2012). "Forebrain organization representing baroreceptor gating of somatosensory afferents within the cortical autonomic network." J. Neurophysiol 108(2): 453-466.

Greaney, J. L., C. E. Schwartz, D. G. Edwards, P. J. Fadel and W. B. Farquhar (2013). "The neural interaction between the arterial baroreflex and muscle metaboreflex is preserved in older men." Exp Physiol 98(10): 1422-1431.

Hagbarth, K. E. and A. B. Vallbo (1968). "Discharge characteristics of human muscle afferents during muscle stretch and contraction." Exp Neurol 22(4): 674-694.

Hurley, K. M., H. Herbert, M. M. Moga and C. B. Saper (1991). "Efferent projections of the infralimbic cortex of the rat." J Comp Neurol 308(2): 249-276.

Jernigan, T. L., S. L. Archibald, C. Fennema-Notestine, A. C. Gamst, J. C. Stout, J. Bonner and J. R. Hesselink (2001). "Effects of age on tissues and regions of the cerebrum and cerebellum." Neurobiol Aging 22(4): 581-594.

Johnson, N. F., C. Kim, J. L. Clasey, A. Bailey and B. T. Gold (2012). "Cardiorespiratory fitness is positively correlated with cerebral white matter integrity in healthy seniors." Neuroimage 59(2): 1514-1523. 
Jones, S. E., B. R. Buchbinder and I. Aharon (2000). "Three-dimensional mapping of cortical thickness using Laplace's equation." Hum Brain Mapp 11(1): 12-32.

Kimmerly, D. S., D. D. O'Leary, R. S. Menon, J. S. Gati and J. K. Shoemaker (2005). "Cortical regions associated with autonomic cardiovascular regulation during lower body negative pressure in humans." J. Physiol 569(Pt 1): 331-345.

King, A. B., R. S. Menon, V. Hachinski and D. F. Cechetto (1999). "Human forebrain activation by visceral stimuli." J Comp Neurol 413(4): 572-582.

Kramer, A. F., K. I. Erickson and S. J. Colcombe (2006). "Exercise, cognition, and the aging brain." J Appl Physiol (1985) 101(4): 1237-1242.

Lambert, E., T. Dawood, M. Schlaich, N. Straznicky, M. Esler and G. Lambert (2008). "Single-unit sympathetic discharge pattern in pathological conditions associated with elevated cardiovascular risk." Clin Exp Pharmacol Physiol 35(4): 503-507.

Macefield, V. G., S. C. Gandevia and L. A. Henderson (2006). "Neural sites involved in the sustained increase in muscle sympathetic nerve activity induced by inspiratory capacity apnea: a fMRI study." J. Appl. Physiol (1985. ) 100(1): 266-273.

Macey, P. M., P. Wu, R. Kumar, J. A. Ogren, H. L. Richardson, M. A. Woo and R. M. Harper (2012). "Differential responses of the insular cortex gyri to autonomic challenges." Auton. Neurosci 168(1-2): 72-81.

Marks, B. L., L. M. Katz, M. Styner and J. K. Smith (2011). "Aerobic fitness and obesity: relationship to cerebral white matter integrity in the brain of active and sedentary older adults." Br J Sports Med 45(15): 1208-1215.

Matthews, S. C., M. P. Paulus, A. N. Simmons, R. A. Nelesen and J. E. Dimsdale (2004). "Functional subdivisions within anterior cingulate cortex and their relationship to autonomic nervous system function." Neuroimage 22(3): 1151-1156.

Meredith, I. T., G. Eisenhofer, G. W. Lambert, E. M. Dewar, G. L. Jennings and M. D. Esler (1993). "Cardiac sympathetic nervous activity in congestive heart failure. Evidence 
for increased neuronal norepinephrine release and preserved neuronal uptake." Circulation 88(1): 136-145.

Monahan, K. D., F. A. Dinenno, D. R. Seals, C. M. Clevenger, C. A. DeSouza and H. Tanaka (2001). "Age-associated changes in cardiovagal baroreflex sensitivity are related to central arterial compliance." Am. J. Physiol Heart Circ. Physiol 281(1): H284-H289.

Mulder, A. B., M. P. Arts and F. H. Lopes da Silva (1997). "Short- and long-term plasticity of the hippocampus to nucleus accumbens and prefrontal cortex pathways in the rat, in vivo." Eur. J. Neurosci 9(8): 1603-1611.

Neafsey, E. J. (1990). "Prefrontal cortical control of the autonomic nervous system: anatomical and physiological observations." Prog Brain Res 85: 147-165; discussion 165146.

Norris, J. W., G. M. Froggatt and V. C. Hachinski (1978). "Cardiac arrhythmias in acute stroke." Stroke 9(4): 392-396.

Norton, K. N., A. Heinecke and J. K. Shoemaker (2015). "The Neuroprotective Effects of Endurance Training on the Aging Brain." Autonomic Neuroscience: Basic and Clinical 192: 110 .

Norton, K. N., T. A. Luchyshyn and S. J. Kevin (2013). "Evidence for a medial prefrontal cortex-hippocampal axis associated with heart rate control in conscious humans." Brain Res 1538: 104-115.

Oppenheimer, S. M., J. X. Wilson, C. Guiraudon and D. F. Cechetto (1991). "Insular cortex stimulation produces lethal cardiac arrhythmias: a mechanism of sudden death?" Brain Res 550(1): 115-121.

Parlow, J., J. P. Viale, G. Annat, R. Hughson and L. Quintin (1995). "Spontaneous cardiac baroreflex in humans. Comparison with drug-induced responses." Hypertension 25(5): 1058-1068. 
Pellman, J. and F. Sheikh (2015). "Atrial fibrillation: mechanisms, therapeutics, and future directions." Compr Physiol 5(2): 649-665.

Peters, J., M. Dauvermann, C. Mette, P. Platen, J. Franke, T. Hinrichs and I. Daum (2009). "Voxel-based morphometry reveals an association between aerobic capacity and grey matter density in the right anterior insula." Neuroscience 163(4): 1102-1108.

Pickering, T. G. and J. Davies (1973). "Estimation of the conduction time of the baroreceptor-cardiac reflex in man." Cardiovasc Res 7(2): 213-219.

Raz, N., F. M. Gunning, D. Head, J. H. Dupuis, J. McQuain, S. D. Briggs, W. J. Loken, A. E. Thornton and J. D. Acker (1997). "Selective aging of the human cerebral cortex observed in vivo: differential vulnerability of the prefrontal gray matter." Cereb. Cortex 7(3): 268-282.

Raz, N., K. M. Rodrigue and E. M. Haacke (2007). "Brain aging and its modifiers: insights from in vivo neuromorphometry and susceptibility weighted imaging." Ann. N. Y. Acad. Sci 1097: 84-93.

Raz, N., A. Williamson, F. Gunning-Dixon, D. Head and J. D. Acker (2000). "Neuroanatomical and cognitive correlates of adult age differences in acquisition of a perceptual-motor skill." Microsc Res Tech 51(1): 85-93.

Schmidt, H., U. Muller-Werdan, T. Hoffmann, D. P. Francis, M. F. Piepoli, M. Rauchhaus, R. Prondzinsky, H. Loppnow, M. Buerke, D. Hoyer and K. Werdan (2005). "Autonomic dysfunction predicts mortality in patients with multiple organ dysfunction syndrome of different age groups." Crit Care Med 33(9): 1994-2002.

Shoemaker, J. K. and R. Goswami (2015). "Forebrain neurocircuitry associated with human reflex cardiovascular control." Front Physiol 6: 240.

Shoemaker, J. K., K. N. Norton, J. Baker and T. Luchyshyn (2015). "Forebrain organization for autonomic cardiovascular control." Auton Neurosci 188: 5-9. 
Soros, P. and V. Hachinski (2012). "Cardiovascular and neurological causes of sudden death after ischaemic stroke." Lancet Neurol 11(2): 179-188.

Soufer, R., J. D. Bremner, J. A. Arrighi, I. Cohen, B. L. Zaret, M. M. Burg and P. Goldman-Rakic (1998). "Cerebral cortical hyperactivation in response to mental stress in patients with coronary artery disease." Proc Natl Acad Sci U S A 95(11): 6454-6459.

Steinback, C. D., D. Salzer, P. J. Medeiros, J. Kowalchuk and J. K. Shoemaker (2009). "Hypercapnic vs. hypoxic control of cardiovascular, cardiovagal, and sympathetic function." Am J Physiol Regul Integr Comp Physiol 296(2): R402-410.

Thayer, J. F. and R. D. Lane (2007). "The role of vagal function in the risk for cardiovascular disease and mortality." Biol Psychol 74(2): 224-242.

Thayer, J. F., J. J. Sollers, 3rd, D. M. Labiner, M. Weinand, A. M. Herring, R. D. Lane and G. L. Ahern (2009). "Age-related differences in prefrontal control of heart rate in humans: a pharmacological blockade study." Int J Psychophysiol 72(1): 81-88.

Thayer, J. F., J. J. Sollers, III, D. M. Labiner, M. Weinand, A. M. Herring, R. D. Lane and G. L. Ahern (2009). "Age-related differences in prefrontal control of heart rate in humans: a pharmacological blockade study." Int. J. Psychophysiol 72(1): 81-88.

van der Kooy, D., L. Y. Koda, J. F. McGinty, C. R. Gerfen and F. E. Bloom (1984). "The organization of projections from the cortex, amygdala, and hypothalamus to the nucleus of the solitary tract in rat." J Comp Neurol 224(1): 1-24.

van der Kooy, D., J. F. McGinty, L. Y. Koda, C. R. Gerfen and F. E. Bloom (1982). "Visceral cortex: a direct connection from prefrontal cortex to the solitary nucleus in rat." Neurosci Lett 33(2): 123-127.

Varma, V. R., Y. Chuang, G. C. Harris, E. J. Tan and M. C. Carlson (2014). "Lowintensity daily walking activity is associated with hippocampal volume in older adults." Hippocampus. 
Verberne, A. J. (1996). "Medullary sympathoexcitatory neurons are inhibited by activation of the medial prefrontal cortex in the rat." Am. J. Physiol 270(4 Pt 2): R713R719.

Verberne, A. J. and N. C. Owens (1998). "Cortical Modulation of theCardiovascular System." Progress in Neurobiology 54(2): 149-168.

Vertes, R. P. (2004). "Differential projections of the infralimbic and prelimbic cortex in the rat." Synapse 51(1): 32-58.

Voss, M. W., S. Heo, R. S. Prakash, K. I. Erickson, H. Alves, L. Chaddock, A. N. Szabo, E. L. Mailey, T. R. Wojcicki, S. M. White, N. Gothe, E. McAuley, B. P. Sutton and A. F. Kramer (2013). "The influence of aerobic fitness on cerebral white matter integrity and cognitive function in older adults: results of a one-year exercise intervention." $\underline{\text { Hum}}$. Brain Mapp 34(11): 2972-2985.

Wesseling, K. H., J. R. Jansen, J. J. Settels and J. J. Schreuder (1993). "Computation of aortic flow from pressure in humans using a nonlinear, three-element model." J Appl Physiol (1985) 74(5): 2566-2573.

Westerhaus, M. J. and A. D. Loewy (2001). "Central representation of the sympathetic nervous system in the cerebral cortex." Brain Res 903(1-2): 117-127.

Winkelmann, T., J. F. Thayer, S. Pohlack, F. Nees, O. Grimm and H. Flor (2016). "Structural brain correlates of heart rate variability in a healthy young adult population." Brain Struct Funct.

Wong, S. W., D. S. Kimmerly, N. Masse, R. S. Menon, D. F. Cechetto and J. K. Shoemaker (2007). "Sex differences in forebrain and cardiovagal responses at the onset of isometric handgrip exercise: a retrospective fMRI study." J. Appl. Physiol 103(4): 1402-1411.

Wong, S. W., N. Masse, D. S. Kimmerly, R. S. Menon and J. K. Shoemaker (2007). "Ventral medial prefrontal cortex and cardiovagal control in conscious humans." Neuroimage 35(2): 698-708. 
Woo, M. A., P. M. Macey, G. C. Fonarow, M. A. Hamilton and R. M. Harper (2003). "Regional brain gray matter loss in heart failure." J Appl Physiol (1985) 95(2): 677-684.

Woo, M. A., S. K. Yadav, P. M. Macey, G. C. Fonarow, R. M. Harper and R. Kumar (2014). "Brain metabolites in autonomic regulatory insular sites in heart failure." $\underline{\mathrm{J} \text { Neurol }}$ Sci 346(1-2): 271-275. 


\section{Chapter 5}

\section{High Cardiorespiratory Fitness in Middle-Age Preserves the Cortical Circuitry Associated with Brain-Heart Integration During Volitional Exercise}

(Submitted to Journal of Neurophysiology July 22, 2016: JN-00592-2016)

\subsection{Introduction}

Rapid increases in HR represent a critical reactive response to physiologic stress, facilitating or sustaining blood pressure and organ perfusion. The tachycardia at the onset of exercise has been linked mechanistically to a rapid reduction in vagal chronotropic control (Hollander and Bouman 1975, Fagraeus and Linnarsson 1976, Mitchell, Reeves et al. 1989). However, although some inter-individual variation exists (Norton, Luchyshyn et al. 2013), advancing age impairs the magnitude of the HR and cardiac output responses at the exercise onset, resulting in a larger sympathetic drive to adjust blood pressure (Lalande, Sawicki et al. 2014). The mechanism(s) affecting the diminished cardiac response with exercise in aging adults remains unknown but may include either changes in autonomic neural adjustments and/or intrinsic cardiac events (Astrand 1960, Bruce, Blackmon et al. 1963, Lester, Sheffield et al. 1968, Dauchot and Gravenstein 1971, Kino, Lance et al. 1975, Seliger, Macek et al. 1978, Sheffield, Maloof et al. 1978, Yin, Spurgeon et al. 1979, Craft and Schwartz 1995, Lalande, Sawicki et al. 2014). Diminished HR variability (O'Brien, O'Hare et al. 1986, Schwartz, Gibb et al. 1991, Agelink, Malessa et al. 2001, Bonnemeier, Richardt et al. 2003, Russoniello, Zhirnov et al. 2013) and a reduced tachycardia following atropine administration (Dauchot and Gravenstein 1971) support the conclusion that age reduces chronic parasympathetic restraint of HR, suggesting that this effect may be a fundamental determinant of impaired cardiac acceleration with exercise in aging adults.

However, the neurological determinants of age-related impairment of cardiovagal function remain an attractive contributor, due to the important role of supramedullary sites on autonomic outflow. Specifically, neuroimaging techniques have enabled 
investigation into a network of cortical regions associated with the autonomic nervous system and cardiovascular control in conscious humans (Critchley, Corfield et al. 2000, Gianaros, Van Der Veen et al. 2004, Williamson 2010, Basnayake, Green et al. 2012, Macey, Wu et al. 2012, Norton, Luchyshyn et al. 2013, Cechetto 2014, Shoemaker, Norton et al. 2014). These regions include the bilateral IC, ACC, PCC, MPFC, and HC. Importantly, experimental studies indicate that the IC, MPFC and HC are of particular relevance to HR control (Burns and Wyss 1985, Ruggiero, Mraovitch et al. 1987, Cechetto and Chen 1990, Yasui, Breder et al. 1991, Oppenheimer, Gelb et al. 1992, Verberne 1996, Fisk and Wyss 1997, Owens and Verberne 2001, Wong, Masse et al. 2007, Goswami, Frances et al. 2012, Norton, Luchyshyn et al. 2013, Shoemaker, Norton et al. 2014). Importantly, advancing age often associates with cortical atrophy (Raz, Lindenberger et al. 2005), changes in brain functional responses (Nyberg, Salami et al. 2010), and declines in cognitive performance (Ronnlund, Nyberg et al. 2005). Notably, the pattern of atrophy begins early in middle age (Raz, Gunning et al. 1997, Raz, Lindenberger et al. 2005, Raz and Rodrigue 2006, Kennedy, Erickson et al. 2009, Raz, Ghisletta et al. 2010) and preferentially affects frontal and parietal regions of the brain, including the MPFC and IC regions: therefore, diminished functional brain-heart associations may be expected in middle aged and older adults.

Nonetheless, substantial inter-individual differences exist in age-related cortical atrophy with some individuals showing resistance to major age-related brain pathology or neurological deficits (Nyberg, Lovden et al. 2012, Pudas, Persson et al. 2013). The determinants of this variability are not known. Of the many possible options, physical activity may be one of the factors affecting this heterogeneity as it demonstrates widespread benefits on brain health in aging individuals, including spared brain volume (Erickson, Prakash et al. 2009, Erickson, Voss et al. 2011, Niemann, Godde et al. 2014, Wood, Nikolov et al. 2016), improved task-related functional brain responses (Colcombe, Kramer et al. 2004, Voelcker-Rehage, Godde et al. 2010), increased white matter integrity (Johnson, Kim et al. 2012, Voss, Heo et al. 2013), and improved cognitive performance (Josefsson, de Luna et al. 2012). However, the functional consequences of this age-related heterogeneity on the forebrain circuitry associated with cardiovascular responses to physiological stress remain unknown. 
The purpose of this study was to examine the impact of cardiorespiratory fitness on brainheart associations in middle aged to older adults. The current study focused on healthy, middle-aged individuals to minimize the confounding variables of senescence and associated co-morbidities, as well as to engage the age-range where cortical vulnerability begins to be expressed and where the effects of advancing age on cardiorespiratory fitness remain weak. With this approach, we tested the hypothesis that high cardiorespiratory fitness improves the HR response to volitional handgrip in middle aged adults, and that such improvement is related to preservation of the entrainment of the MPFC, IC, and HC regions of the cortical autonomic network.

\section{$5.2 \quad$ Methods}

\subsubsection{Participants}

A total of 52 healthy, active individuals participated in this study across a range of fitness and age (26-66mL/kg/min; 45-73 years; 16 female). Table 5.1 provides group characteristics. All participants were non-smokers, free of medications, and without diagnosed hypertension, diabetes, vascular or neurological impairments. Pre-menopausal females were tested during days 1-14 of the menstrual cycle, with day one representing the first day of menstruation. None of the post-menopausal women were on hormone replacement therapy. The University of Western Ontario Health Sciences Ethics Review Board approved this study and adhered to the Declaration of Helsinki. Each participant provided informed, written consent.

\subsubsection{Experimental Design}

Participants completed two separate experimental sessions: 1) physiological recording (LAB session) and 2) functional magnetic resonance neuroimaging session (fMRI; Robarts Research Institute Centre for Functional and Metabolic Imaging). The sessions were performed at the same time of day and separated by a minimum period of 1 week. Participants were familiarized with the experimental procedures prior to their first test session. Participants were instructed to arrive following a $12 \mathrm{~h}$ fast and to refrain from nicotine, alcohol, caffeine, and intense physical exertion for the same duration. Each 
session began with a MVC handgrip calibration, in which the participant was instructed to squeeze a non-magnetic handgrip device connected in series to a pressure transducer (Edwards Lifesciences, PX272, Irvine CA) to their maximal ability while in the supine position. This was repeated twice with the larger value calibrated as $100 \%$. All subjects were right handed and performed the isometric handgrip exercise (IHG) with their dominant hand. During each recording session, visual feedback was provided to the participant of their achieved force in real-time. Baseline data were collected over $5 \mathrm{~min}$ of quiet supine rest. Four repeated bouts each of $40 \%$ MVC force (LAB session), and seven repeated bouts of IHG at $40 \%$ MVC force (fMRI session) were performed, with each contraction lasting $20 \mathrm{sec}$ and separated by $40 \mathrm{sec}$ of rest. The number of trials was increased in the fMRI session to increase the signal-to-noise ratio. The level of perceived exertion produced by the exercise was monitored after each trial on a scale from 6-20 (Borg 1982). None of the participants reported feeling any significant degree of adverse emotional stress or forearm fatigue.

\subsubsection{Assessment of Cardiorespiratory Fitness}

A graded treadmill exercise test, conducted under standard clinical observation, provided information regarding each subject's peak oxygen uptake $\left(\mathrm{VO}_{2 \max }\right)$. During this test, expired air samples were taken at 3-second intervals until the point of volitional exhaustion. Based on the American College of Sports Medicine guidelines (ACSM 1995), $\mathrm{VO}_{2 \max }$ was determined by meeting the following criteria: (1) $\mathrm{VO}_{2}$ ceased to increase with increasing workloads (plateau); (2) heart rate reached the age-predicted maximum value (220-age); and (3) respiratory exchange ratio was > 1.0.

\subsubsection{Physiological Data Acquisition}

During the LAB session, HR was monitored by standard 3-lead ECG techniques. Arterial BP was measured continuously from the finger of the non-exercising left hand, maintained at heart level, by photoplethysmography (Finometer; Finapres Medical Systems B.V. Amsterdam, NL). The BP readings recorded from the Finometer were

corrected against sphygmomanometrically-obtained SBP and DBP pressures that were made intermittently during data collection. 


\subsubsection{Physiological Data Analysis}

All measures were sampled at $1000 \mathrm{~Hz}$, input into a data acquisition board (PowerLab ML795, ADInstruments) for analog-to-digital signal conversion with LabChart7 software (ADInstruments), and stored for offline analysis. HR was calculated from successive R-R intervals obtained from the ECG signal. Beat-by-beat HR data were averaged over $2.5 \mathrm{~s}$ bins (the TR interval for functional scans) and time aligned to ensure a corresponding mean value for each functional scan obtained during the fMRI collection period. The HR response $(\Delta \mathrm{HR})$ to the IHG was determined by averaging the response over the last $20 \mathrm{~s}$ of each rest, and last 10s of each IHG interval. Heart rate responses for each participant were averaged over the four repeated blocks. Blood pressure from the Finometer was converted to MAP using the formula MAP $=1 / 3 \mathrm{SBP}+2 / 3 \mathrm{DBP}$. The Modelflow algorithm provided an index of cardiac output from the finger blood pressure tracings (Wesseling, Jansen et al. 1993). Participant's sex, age, height, and weight were input manually into the Finometer to optimize estimation of cardiac output.

Linear regression analysis examined how cardiorespiratory fitness $\left(\mathrm{VO}_{2 \mathrm{max}}\right)$ predicted $\Delta \mathrm{HR}$ to the IHG task. Although a box-plot analysis revealed several outliers with either a large positive or negative change in HR, removing these individual responses did not change the significance of our regression; therefore, we chose to include them for further analysis. A threshold for significance was set at $\mathrm{p}<0.05$. All regression analyses passed the normality test, as assessed by the Shapiro-Wilk test for normal distribution.

\subsubsection{Neuroimaging Recording Session}

Imaging data were collected using a whole body 3-Tesla imaging system (Magnetom Prisma, Siemens Medical Solutions, Erlangen, Germany) with a 32-channel head coil (Barberi, Gati et al. 2000). A high-resolution $\mathrm{T}_{1}$-weighted structural volume was acquired with a 3D MPRAGE sequence at the beginning of the scanning session (sagittal, matrix 256 X $240 \mathrm{~mm}$, voxel resolution 1.0 X 1.0 X $1.0 \mathrm{~mm}, 1 \mathrm{~mm}$ slice thickness, no gap, flip angle $9^{\circ}, \mathrm{TE}=2.98 \mathrm{~ms}, \mathrm{TI}=900 \mathrm{~ms}, \mathrm{TR}=2.3 \mathrm{~ms}$ ). Transmission and detection of the BOLD contrast signal was acquired by $\mathrm{T}_{2}$-weighted gradient echo-echo planar imaging 
pulse sequence with the following parameters: $\mathrm{TE}=30 \mathrm{~ms}$; FOV $=240 \times 240 \mathrm{~mm}$, flip angle $=90^{\circ}$. Forty-five interleaved axial slices $(3.0 \times 3.0 \mathrm{~mm}$ in-plane voxel resolution, $\mathrm{TR}=2.5 \mathrm{~s}$ ) were acquired in each volume. Five volumes were acquired in the resting participant prior to actual data collection to allow for magnetization equilibrium; these were discarded prior to data analysis. Head movement was limited during the experimental session within a head cradle packed with foam padding, and each subject was instructed to avoid head movements during the scanning period. Beat-by-beat HR was calculated from the continuous signal derived from an MRI-compatible pulse Oximeter (Nonin Medical Inc, 8600FO MRI, Plymouth, MN) placed over the index finger of the non-exercising left hand. In each session, analog signals for pulse recordings and IHG contraction force were sampled at $1000 \mathrm{~Hz}$ with an on-line data acquisition and analysis system (PowerLab, ADInstruments, Mountain View, CA, USA). Respiratory frequency was monitored continuously to prevent Valsalva manoeuvres during the exercise period.

\subsubsection{Neuroimaging Data Analysis}

The $\Delta \mathrm{HR}$ to IHG was determined by averaging the response over the last $20 \mathrm{~s}$ of each rest, and last $10 \mathrm{~s}$ of each IHG interval. Individual HR time courses were determined using $2.5 \mathrm{~s}$ averages of the beat-by-beat HR measures to generate time-aligned data with the BOLD imaging acquisition. HR responses for each participant were averaged over the seven repeated blocks at 40\% MVC. Figure 5.1 displays the average pattern of the HR response to the repeated IHG bouts.

All fMRI data were analyzed using Brain Voyager QX 2.8.4 (Brain Innovation, Maastricht, Netherlands) (Goebel, Esposito et al. 2006). At the first (individual) level, preprocessing included interscan slice acquisition time correction, linear trend removal, temporal high-pass filtering to remove low-frequency drifts, and rigid-body transformation of data to the first acquired image to correct for motion. Individual functional data were co-registered to their respective anatomical template, and subsequently transformed to Talairach space (Talairach and Tournoux 1988). The change in BOLD signal over the exercise period was modeled with a boxcar function 
convolved with a canonical haemodynamic response function and regressed with the individual movement parameters generated during preprocessing. This resulted in subject-specific contrast images containing whole brain information related to sites of both increased and decreased BOLD signal, relative to baseline, during the IHG task as a function of the task itself and the individual HR correlation. The General Linear Model was used to calculate the parameter estimates for all brain voxels (Friston, Holmes et al. 1995). Corrections for multiple comparisons were made using the false discovery rate ( $\mathrm{p}<0.05)$, as well as cluster level threshold estimation (Hagler, Saygin et al. 2006), with 1000 iterations of Monte Carlo simulation and a statistical threshold of $\mathrm{p}<0.05$ for the main task effects. Both corrections were performed sequentially, such that the final results represent only clusters $>10$ voxels in size.

Subsequently, the individual BOLD data for all subjects were "collapsed" resulting in a mean effect estimate per condition. The estimated first level mean effects were analyzed across subjects using a RFX analysis which was performed both in response to the task and the HR regression to assess the consistency of effects between individuals based on the variability of the first-level estimates across subjects. Activation clusters with a minimum size of 300 voxels were converted into voxels of interest (VOI) resulting in a list of the most significant clusters at the whole-brain level. VOI details were transferred to Talairach Client (Research Imaging Institute, version 2.4.3) for the assignment of Talairach coordinates to the nearest grey matter voxel. Subsequently, a manual region of interest analysis was performed for relevant cortical autonomic network regions including the bilateral IC, ACC, PCC, MPFC, and HC, based on earlier data in young individuals performing the same IHG protocol (Wong, Masse et al. 2007, Norton, Luchyshyn et al. 2013). All fMRI data are represented in radiologic convention (i.e. subject's right appears on the left).

To assess the relationship between BOLD responses with our external covariate $\left(\mathrm{VO}_{2 \max }\right)$, we performed a full cortex subtraction (T-test) analysis on high-fit (equal to or $>90^{\text {th }}$ percentile of age-predicted $\left.\mathrm{VO}_{2 \max }\right)$ versus low-fit $\left(<90^{\text {th }}\right.$ percentile of agepredicted $\mathrm{VO}_{2 \max }$ ) individuals. The $90^{\text {th }}$ percentile was the chosen threshold for fitness as it accurately depicts whether individuals were meeting the expected guidelines for their 
age (ACSM 1995), and represents the median fitness level of our sample population. The final correlation maps were adapted by increasing the minimum threshold value $(\mathrm{p}<0.05)$ and adding a cluster threshold $\left(10 \mathrm{~mm}^{2}\right)$.

Task-dependent functional connectivity was assessed using psychophysiological interaction (PPI) analysis (Friston, Buechel et al. 1997). PPI analysis was applied to determine which voxels in the brain increase their relationship with the seed region of interest during the handgrip task, in reference to the HR time course. Based on previous BOLD responses to the handgrip task (Wong, Masse et al. 2007, Norton, Luchyshyn et al. 2013) and our a-priori hypothesis, the MPFC was selected as the seed region. Thus, a task-specific increase in the relationship between brain regions is suggestive of an interaction, or increase in the exchange of information, between the task (psychological factor) and the activation time course of the MPFC (physiological factor). Individual PPI maps were created and subsequently overlaid to obtain an RFX group PPI map.

\subsection{Results}

\subsubsection{Physiological Results}

Baseline anthropometric and cardiovascular characteristics are provided in Table 5.1. The $\triangle \mathrm{HR}$ to the $40 \%$ MVC contraction was the same during the physiological and neuroimaging sessions.

Linear regression revealed a weak relationship between cardiorespiratory fitness $(\mathrm{p}=0.09)$ and the change in HR in response to the IHG task (Figure 5.2).

\subsubsection{Functional (BOLD) Imaging Results: Handgrip Stimulus}

A whole-brain group analysis of the cortical response to IHG revealed seven significant clusters of activation. An increase in BOLD signal relative to baseline was widespread and centered around the left fusiform gyrus, while decreases in activity were observed at the right superior and middle temporal gyrus, right insula, precuneus, left ACC gyrus, and middle temporal gyrus (Figure 5.3). 
Further analysis of our a-priori regions-of-interest revealed increased activity in the bilateral IC and left $\mathrm{HC}$, while reduced activity relative to baseline was observed in the left ACC and PCC, as well as the left MPFC and right HC.

\subsubsection{Functional (BOLD) Imaging Results: Heart Rate}

Whole-brain group analysis of the cortical response regressed with the HR time course revealed six significant clusters of activation. An increase in BOLD signal relative to baseline was observed in the right precentral gyrus and cerebellum, while decreases in activity were observed in the right superior temporal gyrus, as well as the left ACC, parahippocampal gyrus and superior temporal gyrus (Figure 5.4).

Region-of-interest analysis revealed similar results to the task, with increased activity in the bilateral anterior IC and left $\mathrm{HC}$, and reduced activity relative to baseline observed in the left ACC and PCC, as well as the left MPFC and right HC.

\subsubsection{Subtraction Analysis: Low $\mathrm{VO}_{2 \max }-\mathrm{High} \mathrm{VO}_{2 \max }$}

Subtraction analysis of BOLD activation patterns correlated to the HR response between the low-fit and high-fit groups revealed increased activation at the MPFC suggesting that those with a lower $\mathrm{VO}_{2 \max }$ had more activation/less deactivation than those with a high $\mathrm{VO}_{2 m a x}$. Age and the $\Delta \mathrm{HR}$ response to the IHG task were the same between high vs lowfit individuals (Figure 5.5).

\subsubsection{Psychophysiological Interaction Analysis: Heart Rate Time Course}

The PPI approach demonstrated that the MPFC (seed region) engaged in a task-specific increase in the exchange of information with nineteen activated clusters across the wholebrain, relative to the resting condition (Figure 5.6). Within the regions of interest, increased BOLD responses associated with HR connected to the MPFC seed region were observed in the bilateral IC. Decreased information exchange with the MPFC was observed in two clusters at the right middle temporal gyrus and right lingual gyrus (occipital lobe). No connections between the MPFC and HC were observed. 
Table 5.1 Anthropometric and baseline cardiovascular data (mean \pm SD).

\begin{tabular}{rcc}
\hline & Average Group Data & Value Range \\
Age (years) & $59 \pm 8$ & $45-79$ \\
Resting MAP (mmHg) & $87 \pm 9$ & $73-114$ \\
Resting Heart Rate (bpm) & $57 \pm 9$ & $41-83$ \\
BMI (kg/m²) & $25 \pm 3$ & $19-36$ \\
Cardiac Output (L/min) & $6 \pm 2$ & $3-11$ \\
$\Delta \mathrm{HR}(\mathrm{LAB})$ & $5 \pm 3$ & $1-16$ \\
$\Delta \mathrm{HR}(\mathrm{fMRI})$ & $6 \pm 5$ & $-12-22$ \\
$\mathrm{VO}_{2 \max }(\mathrm{mL} / \mathrm{kg} / \mathrm{min})$ & $41 \pm 11$ & $21-66$ \\
$\mathrm{VO}_{2 \max }$ Age Predicted Percentile & $81 \pm 25$ & $25-130$ \\
\hline
\end{tabular}

MAP, mean arterial pressure; $\mathrm{BMI}$, body mass index; $\mathrm{HR}$, heart rate; $\mathrm{VO}_{2 \max }$, maximal oxygen consumption. Age-predicted percentile based on ACSM Guidelines. Cardiac output indexed to body surface area. 
Figure 5.1 Average time course of the heart rate response for the isometric handgrip exercise trials of the neuroimaging session.

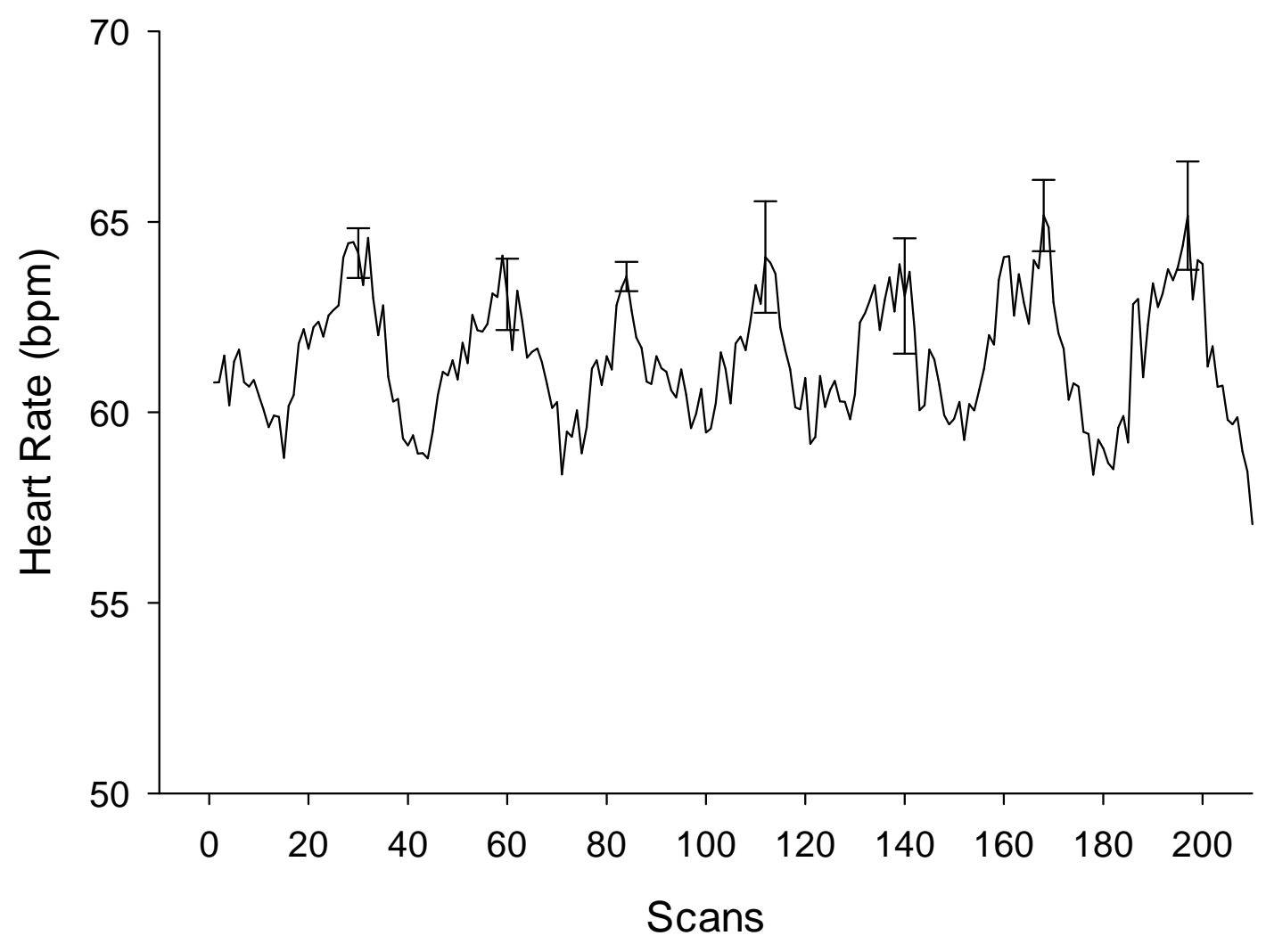

The error bars representing standard deviation are only shown at the end of the exercise for clarity. 
Figure 5.2 Heart Rate (HR) response to isometric handgrip regressed with cardiorespiratory fitness $\left(\mathrm{VO}_{2 \mathrm{max}}\right)$.
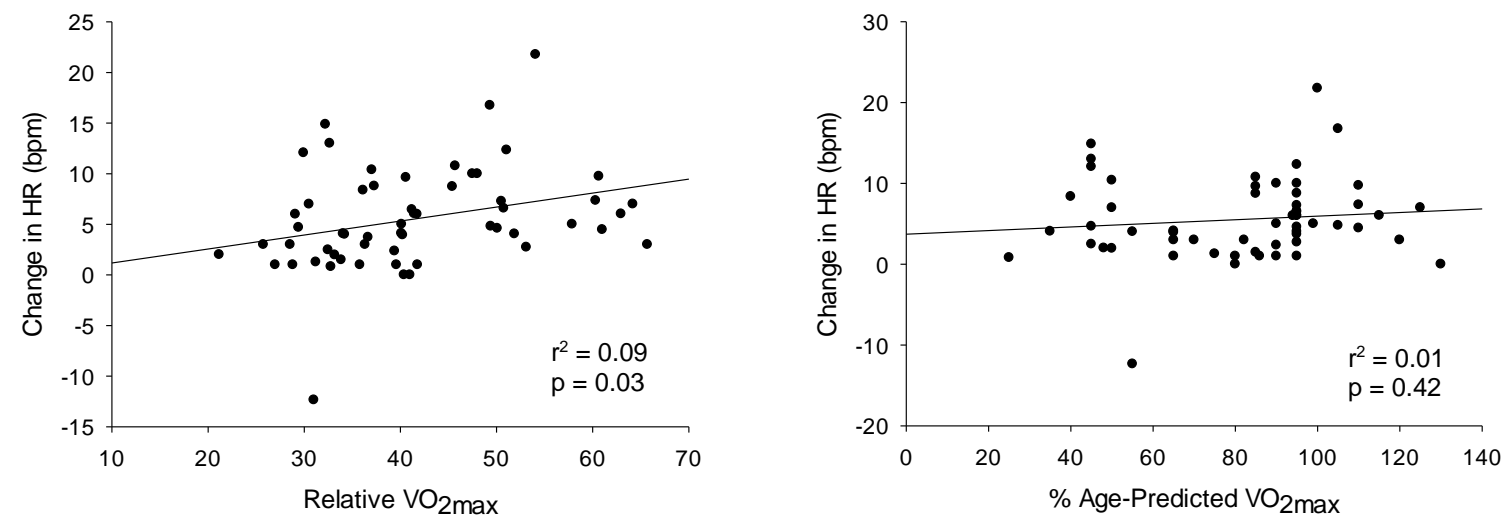
Figure 5.3 BOLD Imaging Results: Handgrip Stimulus.

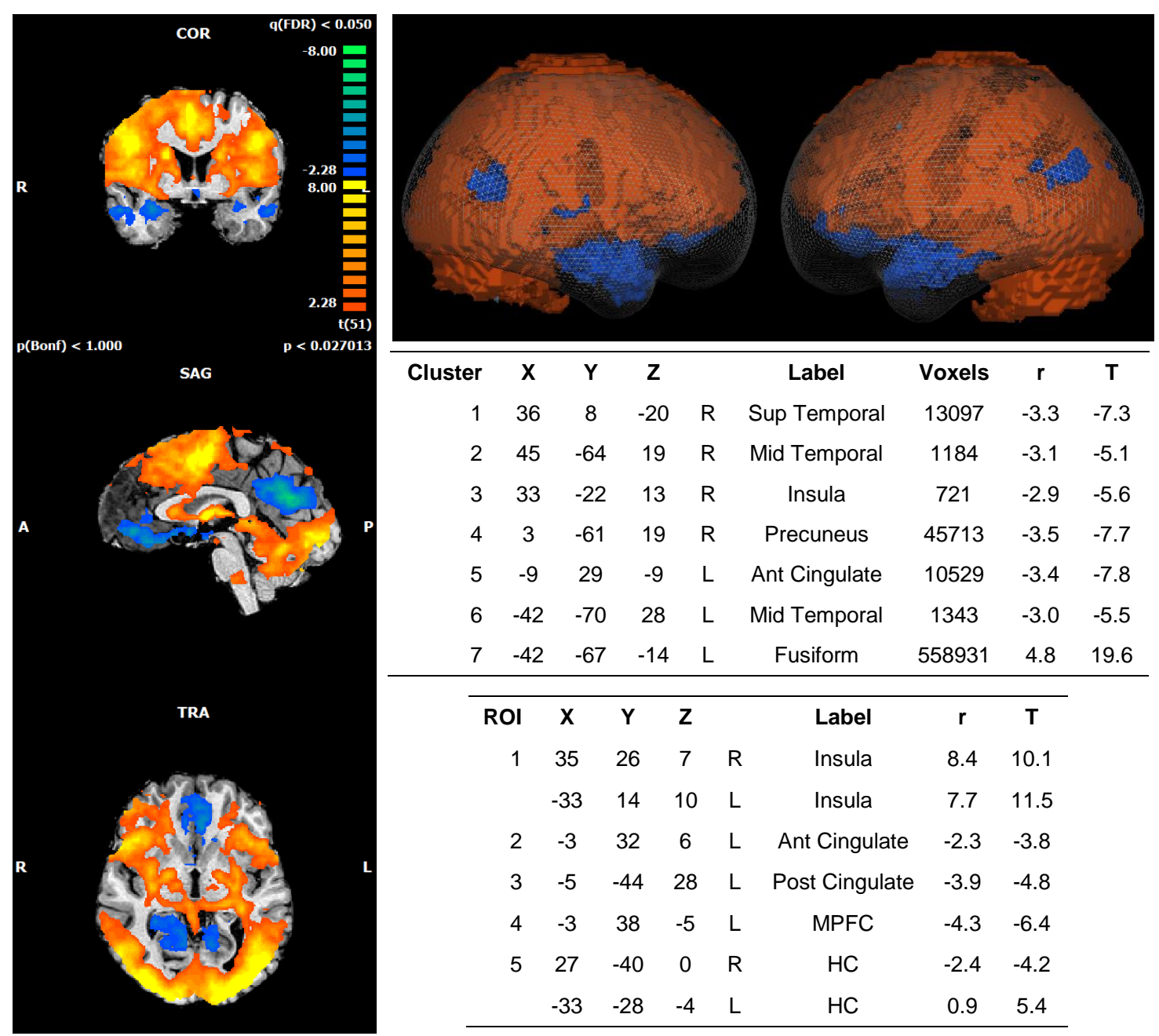

Whole-brain 2D (left panel) and 3D (glass brain, top right panel) representation of activated clusters. Peak cluster (top table) and region of interest details (bottom table) are given with Talairach coordinates. R, right; L, left; Sup, superior; Mid, middle; Ant, anterior; Post, posterior; MPFC, medial prefrontal cortex; HC, hippocampus. r, correlation value; $\mathrm{T}, \mathrm{T}$-score (beta value). Spherical region of interest represents nearest gray matter 257 voxels (same for all ROIs). FDR, $\mathrm{p}<0.05$. Cluster threshold $=10$ voxels. 
Figure 5.4 BOLD Imaging Results: Heart Rate Regressor.
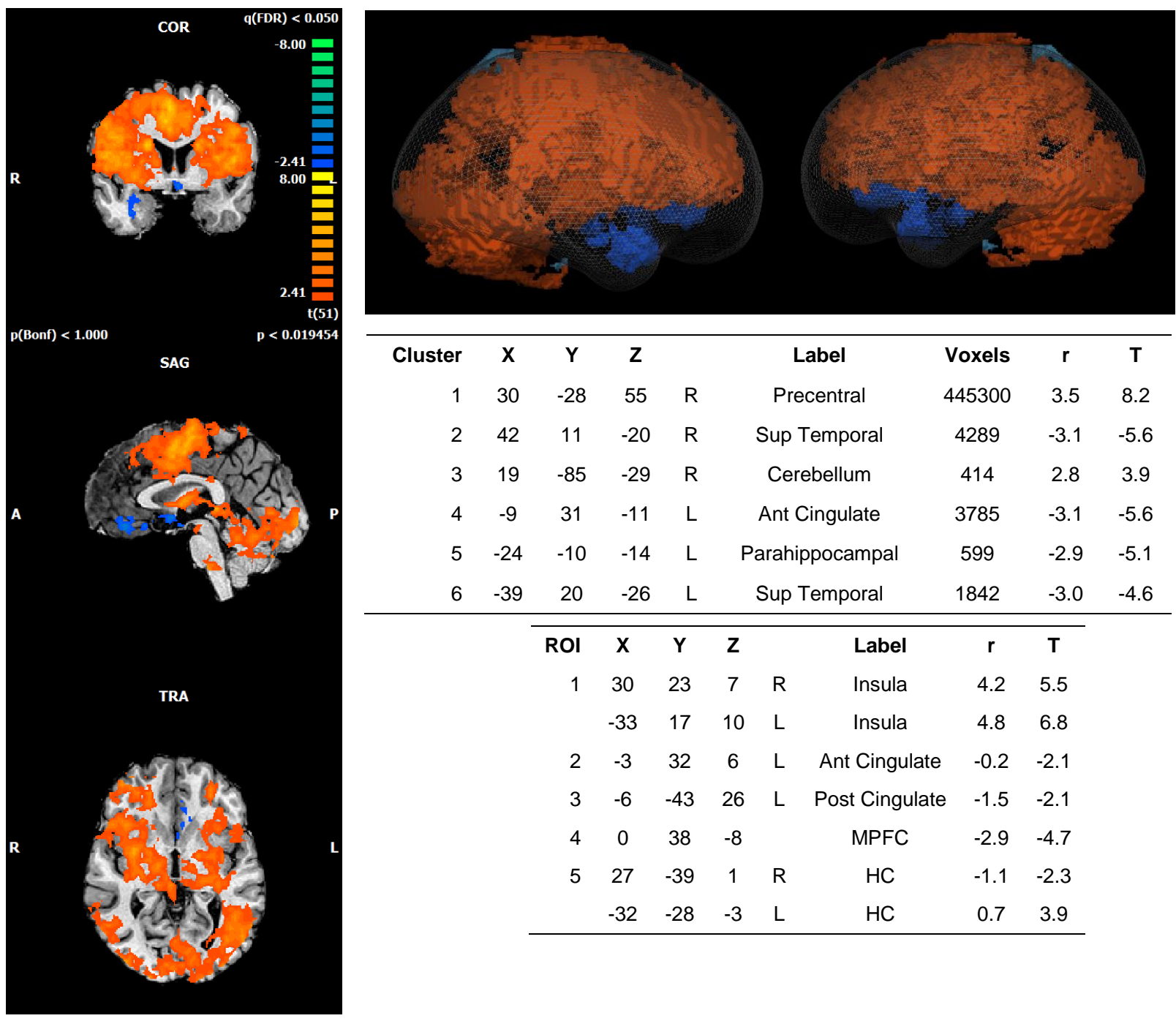

\begin{tabular}{|c|c|c|c|c|c|c|c|c|c|c|}
\hline Cluster & $x$ & $\mathbf{Y}$ & $\mathbf{Z}$ & & \multicolumn{3}{|c|}{ Label } & Voxels & $\mathbf{r}$ & $\mathbf{T}$ \\
\hline 1 & 30 & -28 & 55 & $\mathrm{R}$ & \multicolumn{3}{|c|}{ Precentral } & 445300 & 3.5 & 8.2 \\
\hline 2 & 42 & 11 & -20 & $\mathrm{R}$ & \multicolumn{3}{|c|}{ Sup Temporal } & 4289 & -3.1 & -5.6 \\
\hline 3 & 19 & -85 & -29 & $\mathrm{R}$ & \multicolumn{3}{|c|}{ Cerebellum } & 414 & 2.8 & 3.9 \\
\hline 4 & -9 & 31 & -11 & L & \multicolumn{3}{|c|}{ Ant Cingulate } & 3785 & -3.1 & -5.6 \\
\hline 5 & -24 & -10 & -14 & L & \multicolumn{3}{|c|}{ Parahippocampal } & 599 & -2.9 & -5.1 \\
\hline 6 & -39 & 20 & -26 & L & \multicolumn{3}{|c|}{ Sup Temporal } & 1842 & -3.0 & -4.6 \\
\hline & & ROI & $x$ & $Y$ & $\mathbf{Z}$ & & Label & $\mathbf{r}$ & $\mathbf{T}$ & \\
\hline & & 1 & 30 & 23 & 7 & $\mathrm{R}$ & Insula & 4.2 & 5.5 & \\
\hline & & & -33 & 17 & 10 & L & Insula & 4.8 & 6.8 & \\
\hline & & 2 & -3 & 32 & 6 & $\mathrm{~L}$ & Ant Cingulate & -0.2 & -2.1 & \\
\hline & & 3 & -6 & -43 & 26 & L & Post Cingulate & -1.5 & -2.1 & \\
\hline & & 4 & 0 & 38 & -8 & & MPFC & -2.9 & -4.7 & \\
\hline & & 5 & 27 & -39 & 1 & $\mathrm{R}$ & $\mathrm{HC}$ & -1.1 & -2.3 & \\
\hline & & & -32 & -28 & -3 & L & $\mathrm{HC}$ & 0.7 & 3.9 & \\
\hline
\end{tabular}

Whole-brain 2D (left panel) and 3D (glass brain, top right panel) representation of activated clusters. Peak cluster (top table) and region of interest details (bottom table) are given with Talairach coordinates. R, right; L, left; Sup, superior; Ant, anterior; Post, posterior; MPFC, medial prefrontal cortex; HC, hippocampus. $r$, correlation value; T, Tscore (beta value). Spherical region of interest represents nearest gray matter 257 voxels (same for all ROIs). FDR, $\mathrm{p}<0.05$. Cluster threshold $=10$ voxels. 
Figure 5.5 Subtraction analysis of $\mathrm{Low}^{\mathrm{VO}_{2}}-\mathrm{High} \mathrm{VO}_{2}$ representing the difference in activation patterns that correlate with heart rate.

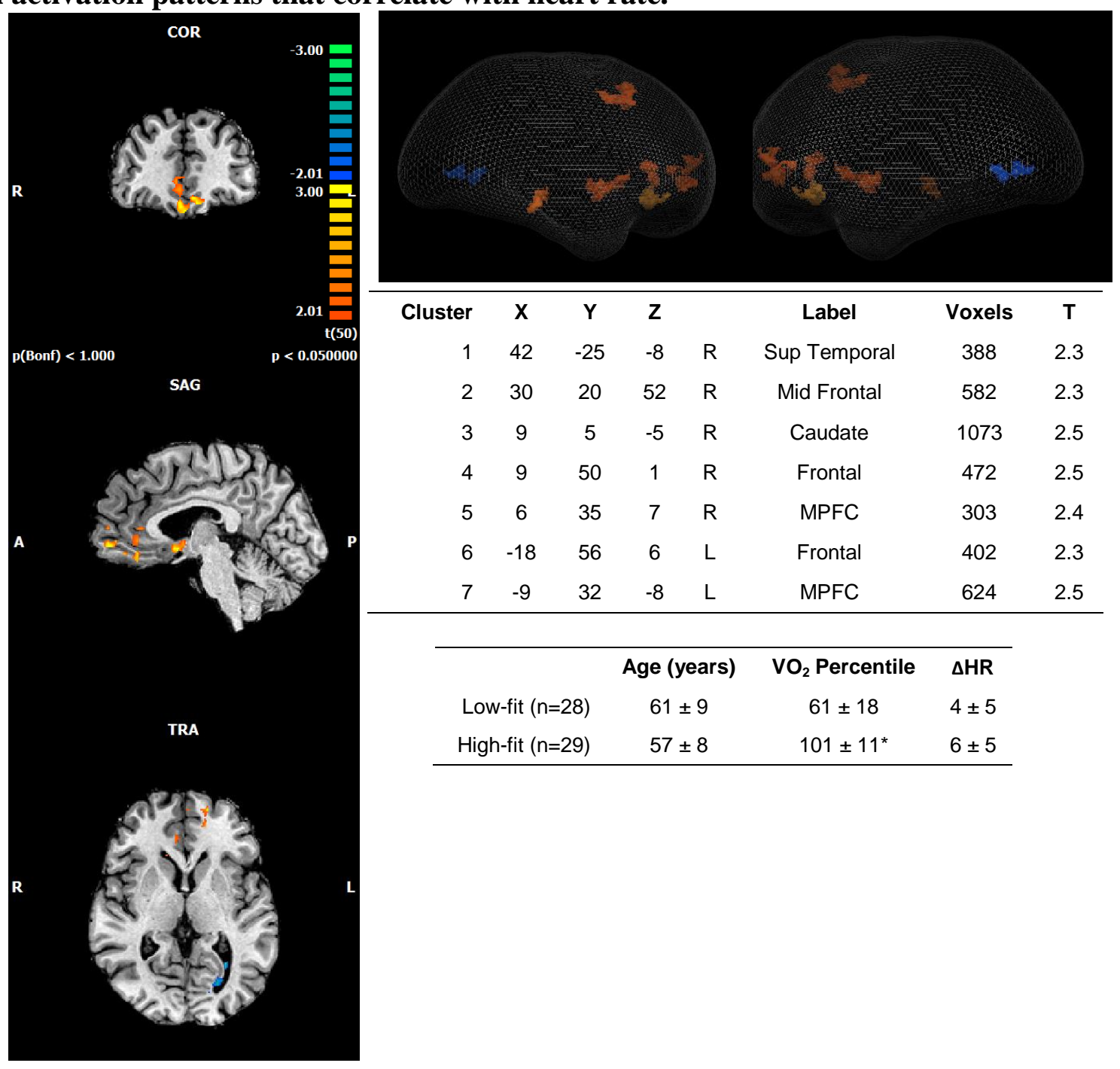

Whole-brain 2D (left panel) and 3D (glass brain, top right panel) representation of activated clusters are shown. Warm colors represent regions of the brain where the low $\mathrm{VO}_{2}$ group has more activation/less deactivation than the high $\mathrm{VO}_{2}$ group. Peak cluster details (top table) are given with Talairach coordinates. Group subject characteristics (bottom table) after assignment based on ACSM $\mathrm{VO}_{2 \max }$ age-predicted percentiles. R, right; L, left; Sup, superior; Mid, middle; MPFC, medial prefrontal cortex; T, beta value representing difference between groups; HR, heart rate. Cluster threshold $=10$ voxels. $*$ different from low-fit, $\mathrm{p}<0.05$. 
Figure 5.6 PPI Results: Functional connectivity of the MPFC during handgrip stimulus regressed with HR time course.

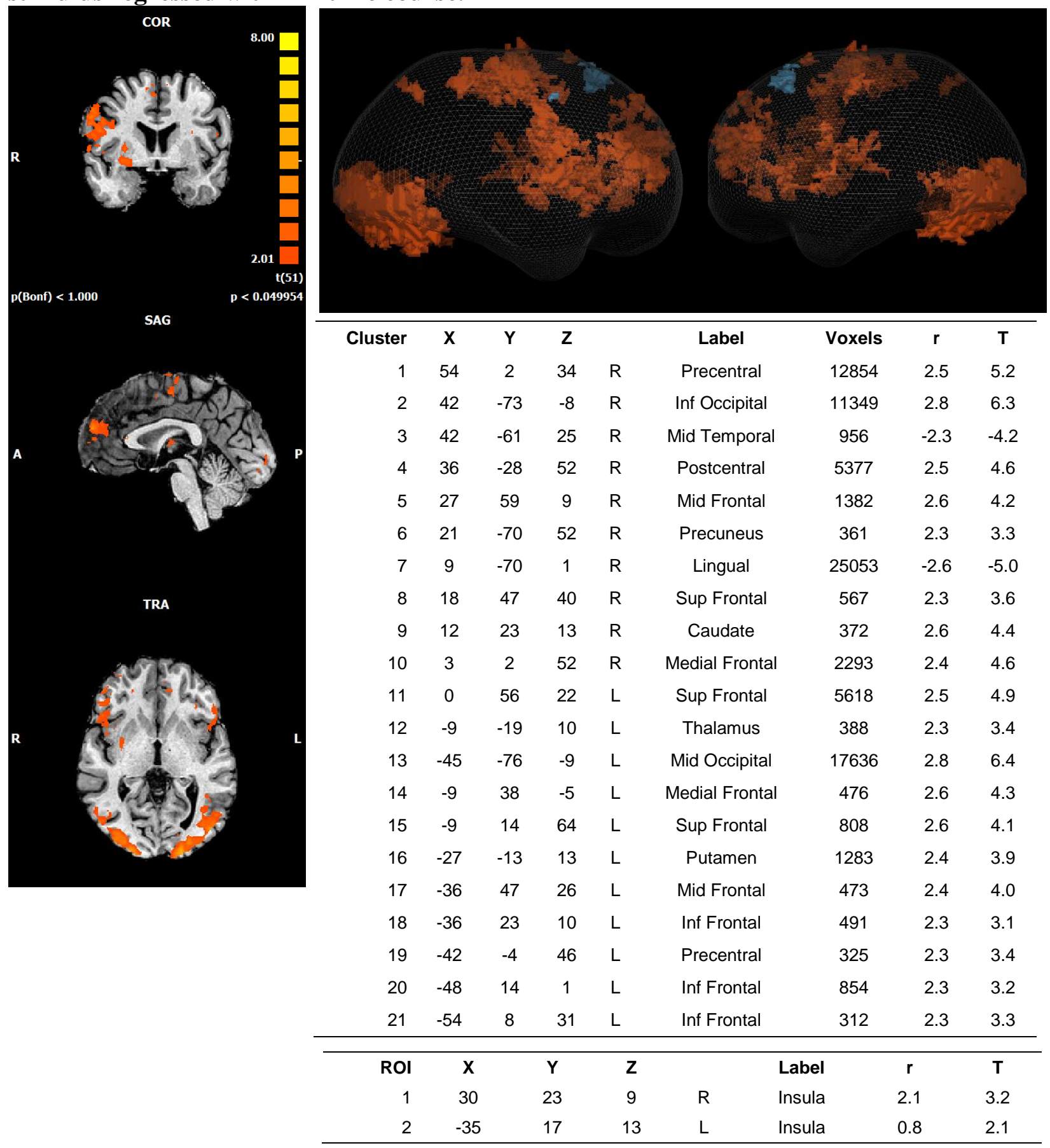

Whole-brain 2D (left panel) and 3D (glass brain, top right panel) representation of activated clusters. Peak cluster (top table) and region of interest details (bottom table) are given with Talairach coordinates. R, right; L, left; Sup, superior; Mid, middle; Inf, inferior. $\mathrm{r}$, correlation value; $\mathrm{T}$, T-score (beta value). Spherical region of interest represents nearest gray matter 257 voxels. Cluster threshold $=10$ voxels, $\mathrm{p}<0.05$. 


\subsection{Discussion}

To our knowledge, the present study represents the first exploration of the relationship between cardiorespiratory fitness and forebrain circuitry associated with cardiovascular control. Our first observation suggests that fitness does not predict the $\Delta \mathrm{HR}$ to a volitional IHG task. Second, as a group, the BOLD response associated with the IHG task, and the with the HR time course, were very similar to those observed in young adults. Finally, in a group-wise contrast, cardiorespiratory fitness predicted the BOLD response associated with $\mathrm{HR}$, but not the $\mathrm{HR}$ response itself. We interpret these findings to suggest that higher levels of fitness positively affect cortical neurocircuitry associated with cardiovascular arousal and that the effect of such neural activity is modulated in the middle to older age range due to factors related to brainstem pathways, and/or end organ responsiveness.

The preservation of deactivation patterns within the MPFC and $\mathrm{HC}$ in the highly fit group of the present study represents an important observation of the current study. These regions were specifically chosen for their known participation in cardiac adjustments to exercise and support the discrete patterns of activation consistently shown in young healthy subjects (Wong, Masse et al. 2007, Goswami, Frances et al. 2011, Norton, Luchyshyn et al. 2013). Further, high cardiorespiratory fitness has been linked to improved hippocampal (Erickson, Voss et al. 2011, Varma, Chuang et al. 2014), medial prefrontal (Colcombe, Erickson et al. 2003, Colcombe, Erickson et al. 2006, Kramer, Erickson et al. 2006), and anterior IC volumes (Peters, Dauvermann et al. 2009). In addition, cross-sectional studies show an association between fitness and brain function(Norton, Heinecke et al. 2015), particularly as it pertains to cognition (Dustman, Ruhling et al. 1984, Prakash, Voss et al. 2011, Voss, Heo et al. 2013, Dupuy, Gauthier et al. 2015, Gauthier, Lefort et al. 2015). The current results add additional novel outcomes that cardiorespiratory fitness positively affects MPFC and HC functional activity patterns to effortful tasks.

Nonetheless, the impact of fitness, while present in forebrain functional patterns to IHG, was not observed in HR responses. The paradoxical observation that fitness did not affect 
the HR response to the IHG stimulus, but did preserve MPFC deactivation in this age group, may be due to challenges associated with the focus on middle-aged adults. Middle to late middle-age represents a period of physiological transition in many physiologic systems, including declines in fitness (Tanaka and Seals 2003) and brain mass (Raz, Ghisletta et al. 2010). Our recent study indicated that a reserve of cortical thickness is developed through higher levels of physical fitness in aging adults (Wood, Nikolov et al. 2016) although the trajectory of cortical atrophy with age persisted. The current results suggest that this cortical reserve induced by higher levels of physical activity may enhance cortical functional patterns as well. Nonetheless, advancing age often produces reductions in intrinsic HR (Craft and Schwartz 1995), and the parasympathomimetic effect of low-dose atropine (Lee, Picard et al. 2008), indicative of non-neural effects of age on cardiac function. Using independent group t-tests, and contrasting the current data with published data from previous research from our laboratory, we sought to further examine the age-dependent relationship between HR responses to IHG and the associated cortical activation patterns. In this retrospective analysis, we established that the current group of moderately-to-highly active individuals was somewhat younger (57 \pm 7 years) that a sedentary group reported previously $(63 \pm 11$ years, p<0.05) (Norton, Luchyshyn et al. 2013) who also generated a significantly lower HR response ( $2 \pm 2 \mathrm{bpm})$ to the same IHG intensity, compared with the current active participants $(6 \pm 5$ bpm, p <0.05). Importantly, the older sedentary group (Norton, Luchyshyn et al. 2013) failed to show MPFC or HC deactivation. Alternatively, young individuals (25 \pm 4 years; $n=17$ ) generated a much larger HR response (>10bpm) (Wong, Masse et al. 2007) with robust MPFC and HC deactivation, and IC activation. Therefore, the current data are consistent with a fitness-based preservation of forebrain activation patterns, but these neurologic benefits have little apparent benefit for HR responses to exercise due, likely, to a local age-related impairment of HR control.

In the current study, the MPFC engaged in significantly stronger connections with several brain regions when compared with baseline activity (Figure 5.6). Importantly, the bilateral IC appeared to correlate with MPFC activity. These results are consistent with known anatomical connections between the IC and MPFC, demonstrated in various 
species (Augustine 1996, Ongur and Price 2000). In addition, our findings confirm the involvement of the IC and MPFC in HR control during effortful tasks previously reported in humans (Williamson, McColl et al. 2003, Wong, Masse et al. 2007), and rodents (Oppenheimer and Cechetto 1990). Age explains at least 10\% of individual differences in functional connectivity (Boraxbekk, Salami et al. 2016) and the HC represents high sensitivity to age-related atrophy (Raz, Lindenberger et al. 2005, Cotman, Berchtold et al. 2007, Raz, Ghisletta et al. 2010). In this regard, the current group may be at the age where $\mathrm{HC}$ involvement in cardiovascular function declines.

\subsection{Limitations}

The current findings are based on cross-sectional brain imaging data. Additional prospective training studies in this age group are needed to see if elevations in cardiovagal function and cardiorespiratory function are linked with corresponding improvements in cortical neurocircuitry related to cardiovascular arousal.

In addition, there are determinants of cardiorespiratory fitness that account for variance beyond habitual physical activity such as genetics, which may account for about half of the variance in individual differences in fitness (Bouchard, An et al. 1999, Bouchard, Sarzynski et al. 2011). To our knowledge, no information exists regarding genetic linkages between brain function and cardiorespiratory function.

The current study focused solely on the HR outcomes during effortful IHG. However, measures of sympathetic nerve activity, also affected by the cortical autonomic network, may expose an additional outcome of interest. In particular, age elevates sympathetic outflow in many individuals (Seals, Taylor et al. 1994, Davy, DeSouza et al. 1998, Tanaka, Dinenno et al. 2000, Monahan, Dinenno et al. 2001, Monahan 2007) but this change can be mitigated by exercise training (Carter and Ray 2015). Moreover, IHG performed by older adults has been characterized by a greater increase in muscle sympathetic nerve activity, compensating for a smaller HR response. Therefore, it may be that the preservation of cortical activation patterns with high fitness in the current study exerted a positive influence over sympathetic outflow, rather than HR. Unfortunately, sympathetic nerve activity was not measured in the current study. 


\subsection{Conclusion}

The current data indicate that high cardiorespiratory fitness sustained through middle-age contributes to the preservation of cortical circuitry associated with cardiovascular control. Overall, these findings support the hypothesis of a role for cardiorespiratory fitness to positively affect the cortical autonomic network, providing new insights into the mechanisms underlying preserved autonomic function and healthy brain aging in adults who engage in long-term regular exercise. 


\subsection{References}

ACSM (1995). Guidelines for Exercise Testing and Prescription. L. W. a. Wilkins. Philadelphia: 160.

Agelink, M. W., R. Malessa, B. Baumann, T. Majewski, F. Akila, T. Zeit and D. Ziegler (2001). "Standardized tests of heart rate variability: normal ranges obtained from 309 healthy humans, and effects of age, gender, and heart rate." Clin Auton Res 11(2): 99108.

Astrand, I. (1960). "Aerobic work capacity in men and women with special reference to age." Acta Physiol Scand Suppl 49(169): 1-92.

Augustine, J. R. (1996). "Circuitry and functional aspects of the insular lobe in primates including humans." Brain Res Brain Res Rev 22(3): 229-244.

Barberi, E. A., J. S. Gati, B. K. Rutt and R. S. Menon (2000). "A transmit-only/receiveonly (TORO) RF system for high-field MRI/MRS applications." Magn Reson. Med 43(2): 284-289.

Basnayake, S. D., A. L. Green and D. J. Paterson (2012). "Mapping the central neurocircuitry that integrates the cardiovascular response to exercise in humans." Exp. Physiol 97(1): 29-38.

Bonnemeier, H., G. Richardt, J. Potratz, U. K. Wiegand, A. Brandes, N. Kluge and H. A. Katus (2003). "Circadian profile of cardiac autonomic nervous modulation in healthy subjects: differing effects of aging and gender on heart rate variability." J Cardiovasc Electrophysiol 14(8): 791-799.

Boraxbekk, C. J., A. Salami, A. Wahlin and L. Nyberg (2016). "Physical activity over a decade modifies age-related decline in perfusion, gray matter volume, and functional connectivity of the posterior default-mode network-A multimodal approach." Neuroimage 131: 133-141. 
Borg, G. (1982). "Ratings of perceived exertion and heart rates during short-term cycle exercise and their use in a new cycling strength test." Int. J. Sports Med 3(3): 153-158.

Bouchard, C., P. An, T. Rice, J. S. Skinner, J. H. Wilmore, J. Gagnon, L. Perusse, A. S. Leon and D. C. Rao (1999). "Familial aggregation of VO(2max) response to exercise training: results from the HERITAGE Family Study." J Appl Physiol (1985) 87(3): 10031008.

Bouchard, C., M. A. Sarzynski, T. K. Rice, W. E. Kraus, T. S. Church, Y. J. Sung, D. C. Rao and T. Rankinen (2011). "Genomic predictors of the maximal O(2) uptake response to standardized exercise training programs." J Appl Physiol (1985) 110(5): 1160-1170.

Bruce, R. A., J. R. Blackmon, J. W. Jones and G. Strait (1963). "Exercising Testing in Adult Normal Subjects and Cardiac Patients." Pediatrics 32: SUPPL 742-756.

Burns, S. M. and J. M. Wyss (1985). "The involvement of the anterior cingulate cortex in blood pressure control." Brain Res 340(1): 71-77.

Carter, J. R. and C. A. Ray (2015). "Sympathetic neural adaptations to exercise training in humans." Auton Neurosci 188: 36-43.

Cechetto, D. F. (2014). "Cortical control of the autonomic nervous system." Exp. Physiol 99(2): 326-331.

Cechetto, D. F. and S. J. Chen (1990). "Subcortical sites mediating sympathetic responses from insular cortex in rats." Am. J. Physiol 258(1 Pt 2): R245-R255.

Colcombe, S. J., K. I. Erickson, N. Raz, A. G. Webb, N. J. Cohen, E. McAuley and A. F. Kramer (2003). "Aerobic fitness reduces brain tissue loss in aging humans." J Gerontol A Biol Sci Med Sci 58(2): 176-180.

Colcombe, S. J., K. I. Erickson, P. E. Scalf, J. S. Kim, R. Prakash, E. McAuley, S. Elavsky, D. X. Marquez, L. Hu and A. F. Kramer (2006). "Aerobic exercise training increases brain volume in aging humans." J Gerontol A Biol Sci Med Sci 61(11): 11661170. 
Colcombe, S. J., A. F. Kramer, K. I. Erickson, P. Scalf, E. McAuley, N. J. Cohen, A. Webb, G. J. Jerome, D. X. Marquez and S. Elavsky (2004). "Cardiovascular fitness, cortical plasticity, and aging." Proc Natl Acad Sci U S A 101(9): 3316-3321.

Cotman, C. W., N. C. Berchtold and L. A. Christie (2007). "Exercise builds brain health: key roles of growth factor cascades and inflammation." Trends Neurosci 30(9): 464-472.

Craft, N. and J. B. Schwartz (1995). "Effects of age on intrinsic heart rate, heart rate variability, and AV conduction in healthy humans." Am J Physiol 268(4 Pt 2): H14411452.

Critchley, H. D., D. R. Corfield, M. P. Chandler, C. J. Mathias and R. J. Dolan (2000). "Cerebral correlates of autonomic cardiovascular arousal: a functional neuroimaging investigation in humans." J. Physiol 523 Pt 1: 259-270.

Dauchot, P. and J. S. Gravenstein (1971). "Effects of atropine on the electrocardiogram in different age groups." Clin Pharmacol Ther 12(2): 274-280.

Davy, K. P., C. A. DeSouza, P. P. Jones and D. R. Seals (1998). "Elevated heart rate variability in physically active young and older adult women." Clin Sci (Lond) 94(6): 579-584.

Dupuy, O., C. J. Gauthier, S. A. Fraser, L. Desjardins-Crepeau, M. Desjardins, S. Mekary, F. Lesage, R. D. Hoge, P. Pouliot and L. Bherer (2015). "Higher levels of cardiovascular fitness are associated with better executive function and prefrontal oxygenation in younger and older women." Front Hum Neurosci 9: 66.

Dustman, R. E., R. O. Ruhling, E. M. Russell, D. E. Shearer, H. W. Bonekat, J. W. Shigeoka, J. S. Wood and D. C. Bradford (1984). "Aerobic exercise training and improved neuropsychological function of older individuals." Neurobiol Aging 5(1): 3542.

Erickson, K. I., R. S. Prakash, M. W. Voss, L. Chaddock, L. Hu, K. S. Morris, S. M. White, T. R. Wojcicki, E. McAuley and A. F. Kramer (2009). "Aerobic fitness is 
associated with hippocampal volume in elderly humans." Hippocampus 19(10): 10301039.

Erickson, K. I., M. W. Voss, R. S. Prakash, C. Basak, A. Szabo, L. Chaddock, J. S. Kim, S. Heo, H. Alves, S. M. White, T. R. Wojcicki, E. Mailey, V. J. Vieira, S. A. Martin, B. D. Pence, J. A. Woods, E. McAuley and A. F. Kramer (2011). "Exercise training increases size of hippocampus and improves memory." Proc Natl Acad Sci U S A 108(7): 3017-3022.

Fagraeus, L. and D. Linnarsson (1976). "Autonomic origin of heart rate fluctuations at the onset of muscular exercise." J. Appl. Physiol 40(5): 679-682.

Fisk, G. D. and J. M. Wyss (1997). "Pressor and depressor sites are intermingled in the cingulate cortex of the rat." Brain Res 754(1-2): 204-212.

Friston, K. J., C. Buechel, G. R. Fink, J. Morris, E. Rolls and R. J. Dolan (1997). "Psychophysiological and modulatory interactions in neuroimaging." Neuroimage 6(3): 218-229.

Friston, K. J., A. P. Holmes, J. B. Poline, P. J. Grasby, S. C. Williams, R. S. Frackowiak and R. Turner (1995). "Analysis of fMRI time-series revisited." Neuroimage 2(1): 45-53.

Gauthier, C. J., M. Lefort, S. Mekary, L. Desjardins-Crepeau, A. Skimminge, P. Iversen, C. Madjar, M. Desjardins, F. Lesage, E. Garde, F. Frouin, L. Bherer and R. D. Hoge (2015). "Hearts and minds: linking vascular rigidity and aerobic fitness with cognitive aging." Neurobiol Aging 36(1): 304-314.

Gianaros, P. J., F. M. Van Der Veen and J. R. Jennings (2004). "Regional cerebral blood flow correlates with heart period and high-frequency heart period variability during working-memory tasks: Implications for the cortical and subcortical regulation of cardiac autonomic activity." Psychophysiology 41(4): 521-530.

Goebel, R., F. Esposito and E. Formisano (2006). "Analysis of functional image analysis contest (FIAC) data with brainvoyager QX: From single-subject to cortically aligned 
group general linear model analysis and self-organizing group independent component analysis." Hum. Brain Mapp 27(5): 392-401.

Goswami, R., M. F. Frances and J. K. Shoemaker (2011). "Representation of somatosensory inputs within the cortical autonomic network." Neuroimage 54(2): 12111220.

Goswami, R., M. F. Frances, C. D. Steinback and J. K. Shoemaker (2012). "Forebrain organization representing baroreceptor gating of somatosensory afferents within the cortical autonomic network." J. Neurophysiol 108(2): 453-466.

Hagler, D. J., Jr., A. P. Saygin and M. I. Sereno (2006). "Smoothing and cluster thresholding for cortical surface-based group analysis of fMRI data." Neuroimage 33(4): 1093-1103.

Hollander, A. P. and L. N. Bouman (1975). "Cardiac acceleration in man elicited by a muscle-heart reflex." J. Appl. Physiol 38(2): 272-278.

Johnson, N. F., C. Kim, J. L. Clasey, A. Bailey and B. T. Gold (2012). "Cardiorespiratory fitness is positively correlated with cerebral white matter integrity in healthy seniors." Neuroimage 59(2): 1514-1523.

Josefsson, M., X. de Luna, S. Pudas, L. G. Nilsson and L. Nyberg (2012). "Genetic and lifestyle predictors of 15-year longitudinal change in episodic memory." J Am Geriatr Soc 60(12): 2308-2312.

Kennedy, K. M., K. I. Erickson, K. M. Rodrigue, M. W. Voss, S. J. Colcombe, A. F. Kramer, J. D. Acker and N. Raz (2009). "Age-related differences in regional brain volumes: a comparison of optimized voxel-based morphometry to manual volumetry." Neurobiol Aging 30(10): 1657-1676.

Kino, M., V. Q. Lance, A. Shahamatpour and D. H. Spodick (1975). "Effects of age on responses to isometric exercise. Isometric handgrip in noninvasive screening for cardiovascular disease." Am Heart J 90(5): 575-581. 
Kramer, A. F., K. I. Erickson and S. J. Colcombe (2006). "Exercise, cognition, and the aging brain." J Appl Physiol (1985) 101(4): 1237-1242.

Lalande, S., C. P. Sawicki, J. R. Baker and J. K. Shoemaker (2014). "Effect of age on the hemodynamic and sympathetic responses at the onset of isometric handgrip exercise." $\underline{\mathbf{J}}$ Appl Physiol (1985) 116(2): 222-227.

Lee, K., G. Picard, S. D. Beske, G. S. Hwang and J. A. Taylor (2008). "Effects of fitness and age on the response to vagotonic atropine." Auton. Neurosci 139(1-2): 60-67.

Lester, M., L. T. Sheffield, P. Trammell and T. J. Reeves (1968). "The effect of age and athletic training on the maximal heart rate during muscular exercise." Am Heart J 76(3): 370-376.

Macey, P. M., P. Wu, R. Kumar, J. A. Ogren, H. L. Richardson, M. A. Woo and R. M. Harper (2012). "Differential responses of the insular cortex gyri to autonomic challenges." Auton. Neurosci 168(1-2): 72-81.

Mitchell, J. H., D. R. Reeves, Jr., H. B. Rogers, N. H. Secher and R. G. Victor (1989). "Autonomic blockade and cardiovascular responses to static exercise in partially curarized man." J. Physiol 413: 433-445.

Monahan, K. D. (2007). "Effect of aging on baroreflex function in humans." Am J Physiol Regul Integr Comp Physiol 293(1): R3-R12.

Monahan, K. D., F. A. Dinenno, D. R. Seals, C. M. Clevenger, C. A. DeSouza and H. Tanaka (2001). "Age-associated changes in cardiovagal baroreflex sensitivity are related to central arterial compliance." Am. J. Physiol Heart Circ. Physiol 281(1): H284-H289.

Niemann, C., B. Godde and C. Voelcker-Rehage (2014). "Not only cardiovascular, but also coordinative exercise increases hippocampal volume in older adults." Front Aging Neurosci 6: 170. 
Norton, K. N., A. Heinecke and J. K. Shoemaker (2015). "The Neuroprotective Effects of Endurance Training on the Aging Brain." Autonomic Neuroscience: Basic and Clinical 192: 110 .

Norton, K. N., T. A. Luchyshyn and S. J. Kevin (2013). "Evidence for a medial prefrontal cortex-hippocampal axis associated with heart rate control in conscious humans." Brain

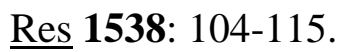

Nyberg, L., M. Lovden, K. Riklund, U. Lindenberger and L. Backman (2012). "Memory aging and brain maintenance." Trends Cogn Sci 16(5): 292-305.

Nyberg, L., A. Salami, M. Andersson, J. Eriksson, G. Kalpouzos, K. Kauppi, J. Lind, S. Pudas, J. Persson and L. G. Nilsson (2010). "Longitudinal evidence for diminished frontal cortex function in aging." Proc Natl Acad Sci U S A 107(52): 22682-22686.

O'Brien, I. A., P. O'Hare and R. J. Corrall (1986). "Heart rate variability in healthy subjects: effect of age and the derivation of normal ranges for tests of autonomic function." Br Heart J 55(4): 348-354.

Ongur, D. and J. L. Price (2000). "The organization of networks within the orbital and medial prefrontal cortex of rats, monkeys and humans." Cereb Cortex 10(3): 206-219.

Oppenheimer, S. M. and D. F. Cechetto (1990). "Cardiac chronotropic organization of the rat insular cortex." Brain Res 533(1): 66-72.

Oppenheimer, S. M., A. Gelb, J. P. Girvin and V. C. Hachinski (1992). "Cardiovascular effects of human insular cortex stimulation." Neurology 42(9): 1727-1732.

Owens, N. C. and A. J. Verberne (2001). "Regional haemodynamic responses to activation of the medial prefrontal cortex depressor region." Brain Res 919(2): 221-231.

Peters, J., M. Dauvermann, C. Mette, P. Platen, J. Franke, T. Hinrichs and I. Daum (2009). "Voxel-based morphometry reveals an association between aerobic capacity and grey matter density in the right anterior insula." Neuroscience 163(4): 1102-1108. 
Prakash, R. S., M. W. Voss, K. I. Erickson, J. M. Lewis, L. Chaddock, E. Malkowski, H. Alves, J. Kim, A. Szabo, S. M. White, T. R. Wojcicki, E. L. Klamm, E. McAuley and A. F. Kramer (2011). "Cardiorespiratory fitness and attentional control in the aging brain." Front Hum Neurosci 4: 229.

Pudas, S., J. Persson, M. Josefsson, X. de Luna, L. G. Nilsson and L. Nyberg (2013). "Brain characteristics of individuals resisting age-related cognitive decline over two decades." J Neurosci 33(20): 8668-8677.

Raz, N., P. Ghisletta, K. M. Rodrigue, K. M. Kennedy and U. Lindenberger (2010). "Trajectories of brain aging in middle-aged and older adults: regional and individual differences." Neuroimage 51(2): 501-511.

Raz, N., F. M. Gunning, D. Head, J. H. Dupuis, J. McQuain, S. D. Briggs, W. J. Loken, A. E. Thornton and J. D. Acker (1997). "Selective aging of the human cerebral cortex observed in vivo: differential vulnerability of the prefrontal gray matter." Cereb. Cortex 7(3): 268-282.

Raz, N., U. Lindenberger, K. M. Rodrigue, K. M. Kennedy, D. Head, A. Williamson, C. Dahle, D. Gerstorf and J. D. Acker (2005). "Regional brain changes in aging healthy adults: general trends, individual differences and modifiers." Cereb. Cortex 15(11): 16761689.

Raz, N. and K. M. Rodrigue (2006). "Differential aging of the brain: patterns, cognitive correlates and modifiers." Neurosci Biobehav Rev 30(6): 730-748.

Ronnlund, M., L. Nyberg, L. Backman and L. G. Nilsson (2005). "Stability, growth, and decline in adult life span development of declarative memory: cross-sectional and longitudinal data from a population-based study." Psychol Aging 20(1): 3-18.

Ruggiero, D. A., S. Mraovitch, A. R. Granata, M. Anwar and D. J. Reis (1987). "A role of insular cortex in cardiovascular function." J. Comp Neurol 257(2): 189-207. 
Russoniello, C. V., Y. N. Zhirnov, V. I. Pougatchev and E. N. Gribkov (2013). "Heart rate variability and biological age: implications for health and gaming." Cyberpsychol Behav Soc Netw 16(4): 302-308.

Schwartz, J. B., W. J. Gibb and T. Tran (1991). "Aging effects on heart rate variation." J Gerontol 46(3): M99-106.

Seals, D. R., J. A. Taylor, A. V. Ng and M. D. Esler (1994). "Exercise and aging: autonomic control of the circulation." Med. Sci. Sports Exerc 26(5): 568-576.

Seliger, V., M. Macek, O. Skranc, J. Horak, J. Piric, P. Handzo, J. Rous and Z. Jirka (1978). "Work capacity of the Czechoslovakian population." Eur J Appl Physiol Occup Physiol 39(3): 155-164.

Sheffield, L. T., J. A. Maloof, J. A. Sawyer and D. Roitman (1978). "Maximal heart rate and treadmill performance of healthy women in relation to age." Circulation 57(1): 7984.

Shoemaker, J. K., K. N. Norton, J. Baker and T. Luchyshyn (2014). "Topic Series: Autonomic responses to exercise: Forebrain organization for autonomic cardiovascular control." Auton. Neurosci.

Talairach, J. and P. Tournoux (1988). Co-Planar Stereotaxic Atlas of the Human Brain. New York, NY, Thieme Medical Publishers Inc.

Tanaka, H., F. A. Dinenno, K. D. Monahan, C. M. Clevenger, C. A. DeSouza and D. R. Seals (2000). "Aging, habitual exercise, and dynamic arterial compliance." Circulation 102(11): 1270-1275.

Tanaka, H. and D. R. Seals (2003). "Invited Review: Dynamic exercise performance in Masters athletes: insight into the effects of primary human aging on physiological functional capacity." J Appl Physiol (1985) 95(5): 2152-2162. 
Varma, V. R., Y. Chuang, G. C. Harris, E. J. Tan and M. C. Carlson (2014). "Lowintensity daily walking activity is associated with hippocampal volume in older adults." Hippocampus.

Verberne, A. J. (1996). "Medullary sympathoexcitatory neurons are inhibited by activation of the medial prefrontal cortex in the rat." Am. J. Physiol 270(4 Pt 2): R713R719.

Voelcker-Rehage, C., B. Godde and U. M. Staudinger (2010). "Physical and motor fitness are both related to cognition in old age." Eur J Neurosci 31(1): 167-176.

Voss, M. W., S. Heo, R. S. Prakash, K. I. Erickson, H. Alves, L. Chaddock, A. N. Szabo, E. L. Mailey, T. R. Wojcicki, S. M. White, N. Gothe, E. McAuley, B. P. Sutton and A. F. Kramer (2013). "The influence of aerobic fitness on cerebral white matter integrity and cognitive function in older adults: results of a one-year exercise intervention." Hum. Brain Mapp 34(11): 2972-2985.

Wesseling, K. H., J. R. Jansen, J. J. Settels and J. J. Schreuder (1993). "Computation of aortic flow from pressure in humans using a nonlinear, three-element model." $\mathrm{J}$ Appl Physiol (1985) 74(5): 2566-2573.

Williamson, J. W. (2010). "The relevance of central command for the neural cardiovascular control of exercise." Exp. Physiol 95(11): 1043-1048.

Williamson, J. W., R. McColl and D. Mathews (2003). "Evidence for central command activation of the human insular cortex during exercise." J. Appl. Physiol (1985. ) 94(5): 1726-1734.

Wong, S. W., N. Masse, D. S. Kimmerly, R. S. Menon and J. K. Shoemaker (2007). "Ventral medial prefrontal cortex and cardiovagal control in conscious humans." Neuroimage 35(2): 698-708.

Wood, K. N., R. Nikolov and J. K. Shoemaker (2016). "Impact of Long-Term Endurance Training Versus Guideline-Based Physical Activity on Brain Structure in Healthy Aging." Frontiers in Aging Neuroscience 8. 
Yasui, Y., C. D. Breder, C. B. Saper and D. F. Cechetto (1991). "Autonomic responses and efferent pathways from the insular cortex in the rat." J. Comp Neurol 303(3): 355374.

Yin, F. C., H. A. Spurgeon, H. L. Greene, E. G. Lakatta and M. L. Weisfeldt (1979). "Age-associated decrease in heart rate response to isoproterenol in dogs." Mech Ageing Dev 10(1-2): 17-25. 


\section{Chapter 6}

\section{General Discussion}

\subsection{Perspectives}

Given the economic, social, and personal burden associated with age-related neural deterioration, identifying cortical sites and patterns of the developing neurologic changes represents a necessary step from which strategies can be developed and tested to prevent declines in structural and functional brain health before they begin. The current observations support a role for sustained cardiorespiratory fitness in the maintenance and preservation of cortical tissue achieved early in life, which may minimize the risk for neurological impairment in senescence and reduce the frailty period of life.

\subsection{Major Findings}

This series of studies examined the potential benefit of sustained cardiorespiratory fitness on brain structure and autonomic function in a healthy human model. Overall, long-term training and high levels of cardiorespiratory fitness elicited, and sustained, greater cortical thickness over a large portion of the brain, with particular emphasis and relevance to the prefrontal cortex. In addition, physical activity preserved activation patterns within the medial prefrontal cortex and delayed the age-related decline in cortical circuitry associated with cardiovascular control. However, life-long exercise training had little effect on the rate of age-related cortical atrophy. In addition, the strength of the relationship between autonomic variables and cortical thickness was determined by age, and was not altered following adjustments for cardiorespiratory fitness. Therefore, the current results illustrate the potent effect of sustained cardiorespiratory fitness to develop cortical reserve beyond that provided by guideline-based activity, but does not eliminate the effect of age on cortical gray matter atrophy.

An initial important observation of this series of studies is the dominant effect of age on autonomic function and brain structure. In our second study, neither life-long exercise training nor guideline-based fitness had an impact on the rate of age-related cortical 
atrophy. Our endurance-trained population showed a significant cortical "reserve" at every age beyond that of the healthy active group, yet the rate of decay with advancing age was still evident, and was not different from our healthy active group. Furthermore, in our third study, we observed that the thickness of the MPFC predicts chronic levels of high HRV and low MSNA; however, this correlation was not affected by inclusion of $\mathrm{VO}_{2 \max }$ in the regression model and was most strongly influenced by age alone.

If these observations reflect deterioration of autonomic cortical structure, the marked agerelated decline and increased variability in the HR responsiveness to IHG becomes a further important observation. Taken together, this series of studies represents not only a continuum of risk for advancing age, but a continuum for fitness and its associated autonomic outcomes (Table 6.1). When compared with previously published data from our laboratory in young healthy, active individuals, the middle and older-aged trained adults in study four demonstrate a diminished HR response to a similar relative IHG tension $(\mathrm{p}<0.005)$. In addition, the older sedentary group from study one demonstrate a further diminished HR response when compared with young ( $\mathrm{p}<0.0001)$. These differences are statistically significant, as determined by an independent group's t-test that contrasted the data from the current studies with those published earlier. This agerelated difference, albeit significant, is not entirely unexpected given what we know about autonomic dysregulation with age. Mechanistically, this IHG protocol is designed to engage exercise-onset reflexive increases in HR that predominantly reflect reduced dominance of parasympathetic control. In this context, the larger the HR response, the greater evidence of efficient parasympathetic control. Therefore, the small HR responses in our older sedentary population, and smaller yet in the coronary artery disease patients, are likely a consequence of age-related impairment of parasympathetic outflow that is further negatively impacted by disease, but may be attenuated with physical fitness. Alternatively, age-dependent changes in the responsiveness of alpha- and betaadrenergic, as well as cardiac muscarinic receptors may also explain this decrease in HR responsiveness and reflex bradycardia (Poller, Nedelka et al. 1997).

Nonetheless, the suppressed HR response in sedentary older adults appears to be consistent with the cortical patterns in response to the IHG task. In our first study, there 
was an absence of deactivation associated with the (lack of) HR response to IHG in the MPFC in older subjects. However, we observed the preservation of expected activity patterns within the MPFC, HC, and bilateral insula in response to both the task, and the HR time course in our active (guideline and trained) middle and older aged groups from study four. These regions were specifically chosen for their known participation in cardiac adjustments to exercise and support the discrete patterns of activation consistently shown in young healthy subjects. Thus, these observations suggest that sustained cardiorespiratory fitness has the capacity to minimize the impact of age on the cortical autonomic circuitry, yet does not completely reverse the age-related neural decline in healthy humans.

Finally, evidence of a dose-response relationship with physical activity appears to exist in the maintenance of cortical mass and cardiovascular control across the middle to older aged period. This pattern raises questions regarding the maximal exercise-induced benefit possible across the adult age span, as well as the appropriate control group for such studies. In our first study, the older sedentary adults did not participate in any physical activity, which was associated with a lower HR response to the IHG task and a lack of cortical activation in expected autonomic regions. When compared to the trained group of the same age, despite a similar $\mathrm{VO}_{2 \max }$, the improved fitness was associated with a larger HR response and preserved cortical response at the MPFC. Furthermore, when compared with the age-matched older sedentary group $(\mathrm{p}<0.05)$, our older trained group showed a preserved HR response equal to that of the middle-aged trained group $(p=0.4)$ suggesting that when physical activity is continued through the early older years, cortical and autonomic function are preserved. Thus, sedentary activity is associated with a significant deterioration of cortical and autonomic function into the older years.

When comparing guideline-based fitness to long-term endurance training, our results from studies two and four indicate that long-term training and higher levels of cardiorespiratory fitness elicit and sustain, greater cortical thickness over a large portion of the brain than those following the age-appropriate guidelines for activity. 
Interestingly, our population of middle and older aged adults, both those following the age appropriate guidelines for activity and those endurance-trained, show no differences in the HR response to the task and a preserved cortical response at the MPFC, despite a significant difference in age and fitness. This result appears to demonstrate a role for continued fitness through the crucial middle to older aged years with respect to preservation of HR control and cortical autonomic patterns, irrespective of activity level. A dose-response relationship between exercise duration/intensity and health-related quality of life has previously been reported, whereby the best outcomes are associated with moderate exercise (Larson, Wang et al. 2006).

However, despite high inter-individual variability, the MPFC factored importantly into the subtraction analyses when correlated with HR illustrating that those subjects with higher fitness (>50\% of age-predicted maximum) had more deactivation than those subjects with lower fitness $(<50 \%)$ in response to the exercise task. Therefore, higher cardiorespiratory fitness achieved through long-term training better preserved the agerelated decline in cortical circuitry associated with cardiovascular control.

Overall, these data suggest that physical activity, beyond the age-appropriate parameters, may be better in terms of cortical structure, but it may only be necessary to follow the minimal recommended guidelines for benefits to be seen with autonomic function. These data engage the debate regarding the health benefits arising from physical fitness versus physical activity (Pogliaghi, Bellotti et al. 2014, Stathokostas, Dogra et al. 2015). In this context, it may be that cortical thickness is associated with the benefits rising from higher fitness whereas autonomic function is sensitive to a lower threshold level of activity.

\subsection{Conclusion}

Taken together, these results form a unique supportive concept towards understanding age-related autonomic impairment. These data illustrate the importance of limbic and forebrain structures in neural control of HR with specific new evidence supporting a link between structural cortical "reserve" and associated cortical function with long-term endurance training. Overall, these findings support the hypothesis of a role for cardiorespiratory fitness to positively alter these networks and brain regions, providing 
new insights into the mechanisms underlying preserved autonomic function and healthy brain aging in adults who engage in long-term regular exercise.

\subsection{References}

Larson, E. B., L. Wang, J. D. Bowen, W. C. McCormick, L. Teri, P. Crane and W. Kukull (2006). "Exercise is associated with reduced risk for incident dementia among persons 65 years of age and older." Ann Intern Med 144(2): 73-81.

Pogliaghi, S., C. Bellotti and D. H. Paterson (2014). ""Tailored" submaximal step test for VO2max prediction in healthy older adults." J Aging Phys Act 22(2): 261-268.

Poller, U., G. Nedelka, J. Radke, K. Ponicke and O. E. Brodde (1997). "Age-dependent changes in cardiac muscarinic receptor function in healthy volunteers." $\mathrm{J}$ Am Coll Cardiol 29(1): 187-193.

Stathokostas, L., S. Dogra and D. H. Paterson (2015). "The independent roles of cardiorespiratory fitness and sedentary time on chronic conditions and Body Mass Index in older adults." J Sports Med Phys Fitness 55(10): 1200-1206. 
Table 6.1 Cortical and physiological results from all studies representing a continuum of risk for advancing age and reduced fitness with associated autonomic outcomes.

\begin{tabular}{|c|c|c|c|c|c|c|}
\hline & $\begin{array}{c}\text { YOUNG } \\
\text { (GUIDELINE) }\end{array}$ & $\begin{array}{l}\text { MIDDLE- } \\
\text { AGED } \\
\text { (GUIDELINE) }\end{array}$ & $\begin{array}{l}\text { MIDDLE- } \\
\text { AGED } \\
\text { (TRAINED) }\end{array}$ & $\begin{array}{c}\text { OLDER } \\
\text { (TRAINED) }\end{array}$ & $\begin{array}{c}\text { OLDER } \\
\text { (SEDENTARY) }\end{array}$ & $\begin{array}{c}\text { CAD } \\
\text { (SEDENTARY) }\end{array}$ \\
\hline $\mathrm{N}$ & 17 & 12 & 41 & 25 & 23 & 17 \\
\hline AGE & $25 \pm 4$ & $58 \pm 9^{*^{+}}$ & $53 \pm 6^{*}$ & $62 \pm 5^{*^{+}}$ & $63 \pm 11^{*^{+}}$ & $59 \pm 9^{*^{+}}$ \\
\hline$\Delta \mathrm{HR}$ & $9 \pm 2$ & $7 \pm 5$ & $5 \pm 5^{*}$ & $6 \pm 4^{*}$ & $4 \pm 2^{*^{\#}}$ & $2 \pm 2^{*}$ \\
\hline $\mathrm{VO}_{2 \max }$ & $49 \pm 8$ & $38 \pm 7^{*^{+}}$ & $55 \pm 10^{*^{\#}}$ & $39 \pm 10^{\star^{+}}$ & $38 \pm 7^{*^{+}}$ & $26 \pm 5^{x^{+}}$ \\
\hline $\begin{array}{c}\text { MPFC } \\
(\downarrow)\end{array}$ & YES & YES & YES & YES & NO & NO \\
\hline IC $(\uparrow)$ & YES & YES & YES & YES & YES & YES \\
\hline $\mathrm{HC}(\downarrow)$ & YES & YES & YES & YES & YES & NO \\
\hline
\end{tabular}

Values are mean $\pm \mathrm{SD}$. $\mathrm{N}=$ sample size; $\Delta \mathrm{HR}=$ change in heart rate to $40 \%$ handgrip task; $\mathrm{VO}_{2 \max }=$ maximal aerobic capacity; $\mathrm{MPFC}=$ medial prefrontal cortex $; \mathrm{IC}=$ insula cortex; HC $=$ hippocampus; $(\downarrow)=$ deactivation; $(\uparrow)=$ activation. $*$ different from young $(\mathrm{p}<0.005) ;+$ different from middle-aged trained $(\mathrm{p}<0.05)$; \# different from older trained $(\mathrm{p}<0.05)$. 


\section{Appendices}

Appendix 1

\begin{tabular}{|c|c|c|c|c|c|}
\hline & TAL X & TAL Y & TAL Z & $\mathbf{r}$ & $\mathbf{p}$ \\
\hline Calcarine Sulcus & -4 & -75 & 15 & -0.689 & 0.00014 \\
\hline Central Sulcus & -35 & -26 & 47 & -0.662 & 0.000311 \\
\hline Cingulate Gyrus & -9 & -45 & 28 & -0.761 & 0.00001 \\
\hline Cingulate Sulcus & -11 & 22 & 28 & -0.742 & 0.000022 \\
\hline Collateral Sulcus & -28 & -35 & -10 & -0.762 & 0.00001 \\
\hline Cuneus & -5 & -77 & 17 & -0.717 & 0.000056 \\
\hline Gyrus Rectus & -8 & 10 & -11 & -0.612 & 0.00116 \\
\hline Inferior Frontal Gyrus & -29 & 50 & 10 & -0.723 & 0.000044 \\
\hline Inferior Frontal Sulcus & -43 & 24 & 30 & -0.676 & 0.00021 \\
\hline Inferior Occipital Gyrus & -28 & -85 & -5 & -0.758 & 0.000012 \\
\hline Inferior Parietal Lobe & -42 & -56 & 39 & -0.720 & 0.000049 \\
\hline Inferior Temporal Gyrus & -48 & -56 & -7 & -0.707 & 0.000079 \\
\hline Inferior Temporal Sulcus & -49 & -6 & -14 & -0.679 & 0.000188 \\
\hline Insula & -32 & 1 & 16 & -0.790 & 0.000003 \\
\hline Intraparietal Sulcus & -26 & -52 & 56 & -0.714 & 0.000062 \\
\hline Lateral Occipitotemporal Gyrus & -40 & -46 & -17 & -0.737 & 0.000027 \\
\hline Lateral Sulcus & -38 & 14 & 12 & -0.761 & 0.00001 \\
\hline Medial Occipitotemporal Gyrus & -11 & -56 & -3 & -0.708 & 0.000075 \\
\hline Medial Prefrontal Cortex & -12 & 42 & 12 & -0.818 & 0.000001 \\
\hline Middle Frontal Gyrus & -27 & 49 & 10 & -0.729 & 0.000036 \\
\hline Middle Occipital Gyrus & -27 & -85 & -4 & -0.675 & 0.000216 \\
\hline Middle Temporal Gyrus & -46 & -1 & -15 & -0.760 & 0.00001 \\
\hline Occipitotemporal Sulcus & -41 & -27 & -17 & -0.672 & 0.000234 \\
\hline Olfactory Sulcus & -7 & 33 & -13 & -0.638 & 0.000608 \\
\hline Orbital Gyri & -18 & 9 & -12 & -0.757 & 0.000012 \\
\hline Orbital Sulci & -16 & 38 & -11 & -0.690 & 0.000133 \\
\hline Parahippocampal Gyrus & -24 & -17 & -21 & -0.693 & 0.000124 \\
\hline Parieto-Occipital Sulcus & -5 & -72 & 35 & -0.632 & 0.0007 \\
\hline Postcentral Gyrus & -49 & -20 & 45 & -0.739 & 0.000024 \\
\hline Postcentral Sulcus & -55 & -18 & 32 & -0.755 & 0.000013 \\
\hline Precentral Gyrus & -37 & -4 & 35 & -0.767 & 0.000008 \\
\hline Precentral Sulcus & -19 & -1 & 57 & 0.730 & 0.000034 \\
\hline Precuneus & -10 & -46 & 27 & -0.770 & 0.000007 \\
\hline Superior Frontal Gyrus & -12 & 42 & 12 & -0.818 & 0.000001 \\
\hline Superior Frontal Sulcus & -21 & 14 & 43 & -0.636 & 0.00063 \\
\hline Superior Occipital Gyrus & -14 & -96 & 9 & -0.747 & 0.000018 \\
\hline Superior Parietal Lobe & -9 & -67 & 44 & -0.662 & 0.000311 \\
\hline Superior Temporal Gyrus & -47 & -9 & -2 & -0.682 & 0.000173 \\
\hline Superior Temporal Sulcus & -52 & -21 & -2 & -0.685 & 0.000156 \\
\hline Supramarginal Gyrus & -52 & -46 & 37 & -0.716 & 0.000056 \\
\hline Transverse Occipital Sulcus & -29 & -69 & 25 & -0.628 & 0.000785 \\
\hline Uncus & -23 & -17 & -21 & -0.697 & 0.000107 \\
\hline
\end{tabular}

Left hemisphere full cortex correlation results on the basis of cortical thickness and burst frequency. 


\begin{tabular}{|c|c|c|c|c|c|}
\hline & TAL $X$ & TAL Y & TAL Z & $\mathbf{r}$ & $\mathbf{p}$ \\
\hline Calcarine Sulcus & -4 & -75 & 15 & -0.727 & 0.000038 \\
\hline Central Sulcus & -35 & -26 & 47 & -0.718 & 0.000053 \\
\hline Cingulate Gyrus & -9 & -46 & 27 & -0.794 & 0.000002 \\
\hline Cingulate Sulcus & -11 & 22 & 28 & -0.773 & 0.000006 \\
\hline Collateral Sulcus & -28 & -35 & -10 & -0.804 & 0.000001 \\
\hline Cuneus & -5 & -77 & 17 & -0.792 & 0.000002 \\
\hline Gyrus Rectus & -4 & 35 & -13 & -0.741 & 0.000022 \\
\hline Inferior Frontal Gyrus & -50 & 18 & 19 & -0.761 & 0.00001 \\
\hline Inferior Frontal Sulcus & -39 & 25 & 24 & -0.725 & 0.000042 \\
\hline Inferior Occipital Gyrus & -28 & -85 & -5 & -0.783 & 0.000004 \\
\hline Inferior Parietal Lobe & -34 & -46 & 41 & -0.730 & 0.000034 \\
\hline Inferior Temporal Gyrus & -48 & -56 & -7 & -0.767 & 0.000008 \\
\hline Inferior Temporal Sulcus & -54 & -25 & -16 & -0.689 & 0.00014 \\
\hline Insula & -32 & 0 & 16 & -0.815 & 0.000001 \\
\hline Intraparietal Sulcus & -19 & -68 & 36 & -0.754 & 0.000013 \\
\hline Lateral Occipitotemporal Gyrus & -40 & -46 & -17 & -0.806 & 0.000001 \\
\hline Lateral Sulcus & -38 & -11 & 18 & -0.780 & 0.000004 \\
\hline Medial Occipitotemporal Gyrus & -11 & -56 & -3 & -0.789 & 0.000003 \\
\hline Medial Prefrontal Cortex & -12 & 42 & 12 & -0.820 & 0.000001 \\
\hline Middle Frontal Gyrus & -27 & 49 & 10 & -0.742 & 0.000022 \\
\hline Middle Occipital Gyrus & -29 & -84 & -4 & -0.716 & 0.000057 \\
\hline Middle Temporal Gyrus & -46 & -1 & -15 & -0.757 & 0.000012 \\
\hline Occipitotemporal Sulcus & -39 & -10 & -24 & -0.708 & 0.000075 \\
\hline Olfactory Sulcus & -7 & 33 & -13 & -0.695 & 0.000115 \\
\hline Orbital Gyri & -18 & 9 & -12 & -0.764 & 0.000009 \\
\hline Orbital Sulci & -16 & 38 & -11 & -0.729 & 0.000035 \\
\hline Parahippocampal Gyrus & -24 & -17 & -21 & -0.717 & 0.000055 \\
\hline Parieto-Occipital Sulcus & -13 & -63 & 48 & -0.705 & 0.000084 \\
\hline Postcentral Gyrus & -5 & -29 & 66 & -0.775 & 0.000005 \\
\hline Postcentral Sulcus & -55 & -18 & 32 & -0.786 & 0.000003 \\
\hline Precentral Gyrus & -46 & 1 & 18 & -0.767 & 0.000008 \\
\hline Precentral Sulcus & -19 & -1 & 57 & 0.749 & 0.000016 \\
\hline Precuneus & -9 & -46 & 27 & -0.794 & 0.000002 \\
\hline Superior Frontal Gyrus & -12 & 42 & 12 & -0.820 & 0.000001 \\
\hline Superior Frontal Sulcus & -22 & 8 & 44 & -0.636 & 0.000631 \\
\hline Superior Occipital Gyrus & -14 & -96 & 9 & -0.791 & 0.000003 \\
\hline Superior Parietal Lobe & -21 & -67 & 30 & -0.704 & 0.000086 \\
\hline Superior Temporal Gyrus & -47 & -9 & -2 & -0.749 & 0.000017 \\
\hline Superior Temporal Sulcus & -52 & -20 & -2 & -0.685 & 0.000156 \\
\hline Supramarginal Gyrus & -46 & -23 & 19 & -0.723 & 0.000045 \\
\hline Transverse Occipital Sulcus & -27 & -70 & 17 & -0.710 & 0.000069 \\
\hline Uncus & -23 & -17 & -21 & -0.719 & 0.00005 \\
\hline
\end{tabular}

Left hemisphere full cortex correlation results on the basis of cortical thickness and burst incidence. 


\begin{tabular}{|c|c|c|c|c|c|}
\hline & TAL X & TAL Y & TAL Z & $\mathbf{r}$ & $\mathbf{p}$ \\
\hline Calcarine Sulcus & -16 & -55 & 6 & 0.458 & 0.000443 \\
\hline Central Sulcus & -34 & -25 & 44 & 0.583 & 0.000003 \\
\hline Cingulate Gyrus & -9 & -43 & 4 & 0.418 & 0.001496 \\
\hline Cingulate Sulcus & -14 & -19 & 39 & 0.509 & 0.000073 \\
\hline Collateral Sulcus & -26 & -67 & -10 & 0.480 & 0.00021 \\
\hline Cuneus & -11 & -94 & 10 & 0.417 & 0.001557 \\
\hline Gyrus Rectus & -5 & 12 & -11 & -0.371 & 0.005315 \\
\hline Inferior Frontal Gyrus & -44 & 37 & -1 & 0.542 & 0.000019 \\
\hline Inferior Frontal Sulcus & -36 & 6 & 37 & 0.573 & 0.000005 \\
\hline Inferior Occipital Gyrus & -27 & -90 & -13 & 0.484 & 0.000179 \\
\hline Inferior Parietal Lobe & -44 & -56 & 22 & 0.420 & 0.001416 \\
\hline Inferior Temporal Gyrus & -36 & 2 & -31 & 0.455 & 0.000478 \\
\hline Inferior Temporal Sulcus & -55 & -51 & -6 & 0.625 & 0 \\
\hline Insula & -27 & 24 & -2 & 0.491 & 0.000141 \\
\hline Intraparietal Sulcus & -29 & -73 & 34 & 0.468 & 0.000316 \\
\hline Lateral Occipitotemporal Gyrus & -36 & 1 & -32 & 0.524 & 0.00004 \\
\hline Lateral Sulcus & -36 & -16 & -3 & 0.528 & 0.000034 \\
\hline Medial Occipitotemporal Gyrus & -18 & -37 & -9 & 0.459 & 0.000429 \\
\hline Medial Prefrontal Cortex & -8 & 53 & 8 & 0.523 & 0.000042 \\
\hline Middle Frontal Gyrus & -32 & 24 & 38 & 0.547 & 0.000016 \\
\hline Middle Occipital Gyrus & -36 & -80 & 10 & 0.393 & 0.002998 \\
\hline Middle Temporal Gyrus & -54 & -50 & -8 & 0.554 & 0.000011 \\
\hline Occipitotemporal Sulcus & -34 & -48 & -16 & 0.341 & 0.010876 \\
\hline Olfactory Sulcus & -5 & 48 & -10 & 0.307 & 0.022668 \\
\hline Orbital Gyri & -27 & 37 & -2 & 0.431 & 0.001015 \\
\hline Orbital Sulci & -27 & 40 & -3 & 0.454 & 0.000504 \\
\hline Parahippocampal Gyrus & -27 & -25 & -17 & -0.331 & 0.0137 \\
\hline Parieto-Occipital Sulcus & -19 & -62 & 18 & 0.576 & 0.000004 \\
\hline Postcentral Gyrus & -35 & -26 & 43 & 0.657 & 0 \\
\hline Postcentral Sulcus & -58 & -17 & 21 & 0.501 & 0.000097 \\
\hline Precentral Gyrus & -51 & -4 & 35 & 0.525 & 0.000039 \\
\hline Precentral Sulcus & -35 & 4 & 36 & 0.602 & 0.000001 \\
\hline Precuneus & -15 & -55 & 7 & 0.508 & 0.000074 \\
\hline Superior Frontal Gyrus & -8 & 53 & 8 & 0.523 & 0.000042 \\
\hline Superior Frontal Sulcus & -19 & 25 & 45 & 0.636 & 0 \\
\hline Superior Occipital Gyrus & -12 & -95 & 9 & 0.401 & 0.002434 \\
\hline Superior Parietal Lobe & -10 & -68 & 44 & 0.517 & 0.000054 \\
\hline Superior Temporal Gyrus & -42 & -27 & 7 & 0.459 & 0.000424 \\
\hline Superior Temporal Sulcus & -50 & -10 & -7 & 0.481 & 0.000203 \\
\hline Supramarginal Gyrus & -52 & -45 & 39 & 0.678 & 0 \\
\hline Transverse Occipital Sulcus & -31 & -77 & 19 & 0.391 & 0.003129 \\
\hline Uncus & -24 & -14 & -20 & 0.179 & 0.191934 \\
\hline
\end{tabular}

Left hemisphere full cortex correlation results on the basis of cortical thickness and baroreflex sensitivity. 


\begin{tabular}{|c|c|c|c|c|c|}
\hline & TAL X & TAL Y & TAL Z & $\mathbf{r}$ & $\mathbf{p}$ \\
\hline Calcarine Sulcus & -19 & -58 & 6 & 0.418 & 0.00186 \\
\hline Central Sulcus & -11 & -32 & 67 & -0.388 & 0.004108 \\
\hline Cingulate Gyrus & -2 & -8 & 28 & 0.481 & 0.000265 \\
\hline Cingulate Sulcus & -9 & 36 & 20 & 0.516 & 0.000076 \\
\hline Collateral Sulcus & -27 & -68 & -12 & 0.445 & 0.000833 \\
\hline Cuneus & -21 & -85 & 24 & 0.474 & 0.000335 \\
\hline Gyrus Rectus & -7 & 11 & -12 & 0.341 & 0.012594 \\
\hline Inferior Frontal Gyrus & -44 & 37 & -1 & 0.484 & 0.000244 \\
\hline Inferior Frontal Sulcus & -39 & 13 & 32 & 0.458 & 0.000555 \\
\hline Inferior Occipital Gyrus & -14 & -88 & -17 & 0.400 & 0.002966 \\
\hline Inferior Parietal Lobe & -33 & -46 & 40 & 0.429 & 0.001364 \\
\hline Inferior Temporal Gyrus & -50 & -13 & -23 & 0.449 & 0.000754 \\
\hline Inferior Temporal Sulcus & -55 & -38 & -11 & 0.349 & 0.010508 \\
\hline Insula & -30 & 11 & 16 & 0.499 & 0.000145 \\
\hline Intraparietal Sulcus & -42 & -54 & 39 & 0.403 & 0.002762 \\
\hline Lateral Occipitotemporal Gyrus & -36 & 1 & -32 & 0.387 & 0.004193 \\
\hline Lateral Sulcus & -37 & -14 & -5 & 0.482 & 0.00026 \\
\hline Medial Occipitotemporal Gyrus & -12 & -87 & -14 & 0.423 & 0.001615 \\
\hline Medial Prefrontal Cortex & -8 & 51 & 8 & 0.458 & 0.000565 \\
\hline Middle Frontal Gyrus & -26 & 43 & 23 & 0.486 & 0.000225 \\
\hline Middle Occipital Gyrus & -30 & -83 & -3 & 0.384 & 0.004544 \\
\hline Middle Temporal Gyrus & -49 & -26 & -1 & 0.484 & 0.000239 \\
\hline Occipitotemporal Sulcus & -46 & -23 & -20 & 0.382 & 0.004807 \\
\hline Olfactory Sulcus & -9 & 27 & -12 & 0.410 & 0.002293 \\
\hline Orbital Gyri & -13 & 17 & -14 & 0.390 & 0.003927 \\
\hline Orbital Sulci & -32 & 47 & -3 & 0.403 & 0.002771 \\
\hline Parahippocampal Gyrus & -33 & 2 & -33 & 0.321 & 0.018904 \\
\hline Parieto-Occipital Sulcus & -8 & -59 & 48 & 0.460 & 0.000537 \\
\hline Postcentral Gyrus & -48 & -17 & 31 & 0.474 & 0.00034 \\
\hline Postcentral Sulcus & -37 & -34 & 40 & 0.485 & 0.000231 \\
\hline Precentral Gyrus & -37 & -14 & 52 & 0.410 & 0.002274 \\
\hline Precentral Sulcus & -33 & -9 & 46 & 0.442 & 0.000929 \\
\hline Precuneus & -12 & -45 & 29 & 0.410 & 0.002286 \\
\hline Superior Frontal Gyrus & -7 & 50 & 38 & 0.493 & 0.000177 \\
\hline Superior Frontal Sulcus & -19 & 25 & 45 & 0.533 & 0.000041 \\
\hline Superior Occipital Gyrus & -22 & -82 & 23 & 0.592 & 0.000003 \\
\hline Superior Parietal Lobe & -9 & -45 & 63 & -0.442 & 0.000919 \\
\hline Superior Temporal Gyrus & -48 & 2 & -6 & 0.404 & 0.002703 \\
\hline Superior Temporal Sulcus & -48 & -25 & -2 & 0.514 & 0.000084 \\
\hline Supramarginal Gyrus & -52 & -45 & 39 & 0.624 & 0.000001 \\
\hline Transverse Occipital Sulcus & -35 & -81 & 24 & 0.418 & 0.001847 \\
\hline Uncus & -23 & -15 & -21 & 0.223 & 0.108889 \\
\hline
\end{tabular}

Left hemisphere full cortex correlation results on the basis of cortical thickness and In-high frequency. 


\begin{tabular}{|c|c|c|c|c|c|}
\hline & TAL X & TAL Y & TAL Z & $\mathbf{r}$ & $\mathbf{p}$ \\
\hline Calcarine Sulcus & -14 & -45 & -1 & 0.279 & 0.043223 \\
\hline Central Sulcus & -34 & -25 & 44 & 0.435 & 0.001145 \\
\hline Cingulate Gyrus & -4 & -23 & 29 & 0.391 & 0.003826 \\
\hline Cingulate Sulcus & -14 & -19 & 39 & 0.414 & 0.002056 \\
\hline Collateral Sulcus & -28 & -38 & -8 & 0.409 & 0.002336 \\
\hline Cuneus & -22 & -84 & 23 & 0.456 & 0.000599 \\
\hline Gyrus Rectus & -5 & 12 & -11 & -0.356 & 0.008793 \\
\hline Inferior Frontal Gyrus & -45 & 35 & -1 & 0.423 & 0.001619 \\
\hline Inferior Frontal Sulcus & -40 & 14 & 33 & 0.471 & 0.00037 \\
\hline Inferior Occipital Gyrus & -46 & -63 & -1 & 0.327 & 0.016955 \\
\hline Inferior Parietal Lobe & -41 & -55 & 38 & 0.358 & 0.008551 \\
\hline Inferior Temporal Gyrus & -52 & 0 & -24 & 0.471 & 0.000366 \\
\hline Inferior Temporal Sulcus & -55 & -48 & -9 & 0.393 & 0.003608 \\
\hline Insula & -34 & -6 & 12 & 0.432 & 0.001246 \\
\hline Intraparietal Sulcus & -22 & -62 & 53 & 0.392 & 0.003672 \\
\hline Lateral Occipitotemporal Gyrus & -36 & 2 & -31 & 0.362 & 0.007818 \\
\hline Lateral Sulcus & -46 & 35 & -1 & 0.438 & 0.001052 \\
\hline Medial Occipitotemporal Gyrus & -14 & -51 & -3 & 0.414 & 0.002055 \\
\hline Medial Prefrontal Cortex & -11 & 42 & 9 & 0.436 & 0.001093 \\
\hline Middle Frontal Gyrus & -26 & 43 & 23 & 0.493 & 0.000174 \\
\hline Middle Occipital Gyrus & -28 & -84 & -2 & 0.296 & 0.031384 \\
\hline Middle Temporal Gyrus & -49 & -26 & -1 & 0.432 & 0.001228 \\
\hline Occipitotemporal Sulcus & -44 & -25 & -18 & 0.288 & 0.036585 \\
\hline Olfactory Sulcus & -14 & 18 & -12 & 0.304 & 0.026875 \\
\hline Orbital Gyri & -13 & 17 & -14 & 0.397 & 0.003287 \\
\hline Orbital Sulci & -41 & 33 & -7 & 0.320 & 0.019603 \\
\hline Parahippocampal Gyrus & -33 & 2 & -33 & 0.312 & 0.023097 \\
\hline Parieto-Occipital Sulcus & -14 & -73 & 44 & 0.413 & 0.002105 \\
\hline Postcentral Gyrus & -36 & -26 & 42 & 0.476 & 0.000317 \\
\hline Postcentral Sulcus & -37 & -34 & 40 & 0.462 & 0.000493 \\
\hline Precentral Gyrus & -9 & -27 & 70 & -0.366 & 0.00704 \\
\hline Precentral Sulcus & -33 & -9 & 46 & 0.476 & 0.000313 \\
\hline Precuneus & -6 & -52 & 10 & 0.347 & 0.010837 \\
\hline Superior Frontal Gyrus & -18 & 24 & 48 & 0.479 & 0.000288 \\
\hline Superior Frontal Sulcus & -19 & 35 & 43 & 0.506 & 0.00011 \\
\hline Superior Occipital Gyrus & -22 & -82 & 23 & 0.521 & 0.000063 \\
\hline Superior Parietal Lobe & -14 & -72 & 45 & 0.440 & 0.000978 \\
\hline Superior Temporal Gyrus & -50 & -35 & 11 & 0.483 & 0.000248 \\
\hline Superior Temporal Sulcus & -48 & -25 & -2 & 0.446 & 0.000817 \\
\hline Supramarginal Gyrus & -52 & -43 & 39 & 0.619 & 0.000001 \\
\hline Transverse Occipital Sulcus & -35 & -80 & 25 & 0.342 & 0.01227 \\
\hline Uncus & -24 & -14 & -20 & 0.158 & 0.258131 \\
\hline
\end{tabular}

Left hemisphere full cortex correlation results on the basis of cortical thickness and SD1. 


\begin{tabular}{|c|c|c|c|c|c|}
\hline & TAL X & TAL Y & TAL Z & $\mathbf{r}$ & $\mathbf{p}$ \\
\hline Calcarine Sulcus & -8 & -73 & 8 & -0.427 & 0.001411 \\
\hline Central Sulcus & -43 & -13 & 32 & 0.463 & 0.000484 \\
\hline Cingulate Gyrus & -6 & 36 & 7 & 0.554 & 0.000017 \\
\hline Cingulate Sulcus & -7 & 37 & 14 & 0.440 & 0.000989 \\
\hline Collateral Sulcus & -27 & -68 & -12 & 0.409 & 0.00234 \\
\hline Cuneus & -22 & -84 & 23 & 0.550 & 0.00002 \\
\hline Gyrus Rectus & -5 & 12 & -11 & -0.336 & 0.01396 \\
\hline Inferior Frontal Gyrus & -45 & 35 & -1 & 0.411 & 0.002253 \\
\hline Inferior Frontal Sulcus & -40 & 14 & 33 & 0.434 & 0.001151 \\
\hline Inferior Occipital Gyrus & -40 & -65 & -11 & 0.382 & 0.004725 \\
\hline Inferior Parietal Lobe & -42 & -56 & 39 & 0.446 & 0.000806 \\
\hline Inferior Temporal Gyrus & -50 & -13 & -23 & 0.462 & 0.00049 \\
\hline Inferior Temporal Sulcus & -55 & -39 & -11 & 0.390 & 0.003897 \\
\hline Insula & -33 & -5 & 12 & 0.420 & 0.001746 \\
\hline Intraparietal Sulcus & -30 & -46 & 53 & 0.454 & 0.000644 \\
\hline Lateral Occipitotemporal Gyrus & -47 & -24 & -20 & 0.472 & 0.000362 \\
\hline Lateral Sulcus & -37 & -14 & -5 & 0.447 & 0.000801 \\
\hline Medial Occipitotemporal Gyrus & -10 & -87 & -15 & 0.371 & 0.006177 \\
\hline Medial Prefrontal Cortex & -6 & 36 & 7 & 0.554 & 0.000017 \\
\hline Middle Frontal Gyrus & -26 & 43 & 23 & 0.525 & 0.000054 \\
\hline Middle Occipital Gyrus & -29 & -83 & -2 & 0.348 & 0.010631 \\
\hline Middle Temporal Gyrus & -48 & -36 & 3 & 0.474 & 0.000333 \\
\hline Occipitotemporal Sulcus & -46 & -23 & -20 & 0.474 & 0.000335 \\
\hline Olfactory Sulcus & -9 & 32 & -12 & 0.293 & 0.03327 \\
\hline Orbital Gyri & -21 & 46 & -4 & 0.440 & 0.000964 \\
\hline Orbital Sulci & -19 & 48 & -4 & 0.434 & 0.001175 \\
\hline Parahippocampal Gyrus & -33 & 2 & -33 & 0.356 & 0.008943 \\
\hline Parieto-Occipital Sulcus & -8 & -59 & 48 & 0.350 & 0.010235 \\
\hline Postcentral Gyrus & -42 & -13 & 31 & 0.467 & 0.000429 \\
\hline Postcentral Sulcus & -37 & -34 & 40 & 0.505 & 0.000116 \\
\hline Precentral Gyrus & -30 & -11 & 46 & 0.361 & 0.007959 \\
\hline Precentral Sulcus & -33 & -9 & 46 & 0.441 & 0.000962 \\
\hline Precuneus & -8 & -56 & 28 & 0.470 & 0.000389 \\
\hline Superior Frontal Gyrus & -10 & 63 & 5 & 0.546 & 0.000024 \\
\hline Superior Frontal Sulcus & -19 & 25 & 45 & 0.495 & 0.000162 \\
\hline Superior Occipital Gyrus & -22 & -82 & 23 & 0.639 & 0 \\
\hline Superior Parietal Lobe & -27 & -46 & 53 & 0.476 & 0.000311 \\
\hline Superior Temporal Gyrus & -51 & -36 & 11 & 0.407 & 0.002517 \\
\hline Superior Temporal Sulcus & -48 & -25 & -4 & 0.478 & 0.000294 \\
\hline Supramarginal Gyrus & -52 & -43 & 39 & 0.578 & 0.000006 \\
\hline Transverse Occipital Sulcus & -36 & -80 & 24 & 0.331 & 0.015412 \\
\hline Uncus & -23 & -16 & -21 & 0.217 & 0.119327 \\
\hline
\end{tabular}

Left hemisphere full cortex correlation results on the basis of cortical thickness and SDNN. 


\begin{tabular}{|c|c|c|c|c|c|}
\hline & TAL X & TAL Y & TAL Z & $\mathbf{r}$ & $\mathbf{p}$ \\
\hline Calcarine Sulcus & -7 & -71 & 8 & -0.443 & 0.00089 \\
\hline Central Sulcus & -43 & -13 & 32 & 0.496 & 0.000162 \\
\hline Cingulate Gyrus & -6 & 36 & 7 & 0.561 & 0.000012 \\
\hline Cingulate Sulcus & -17 & -39 & 40 & 0.420 & 0.001737 \\
\hline Collateral Sulcus & -21 & -84 & -15 & 0.433 & 0.001198 \\
\hline Cuneus & -22 & -84 & 23 & 0.592 & 0.000003 \\
\hline Gyrus Rectus & -5 & 12 & -11 & -0.342 & 0.012231 \\
\hline Inferior Frontal Gyrus & -45 & 35 & -1 & 0.446 & 0.000815 \\
\hline Inferior Frontal Sulcus & -40 & 14 & 33 & 0.437 & 0.001069 \\
\hline Inferior Occipital Gyrus & -41 & -59 & -14 & 0.383 & 0.0047 \\
\hline Inferior Parietal Lobe & -41 & -55 & 38 & 0.500 & 0.000137 \\
\hline Inferior Temporal Gyrus & -36 & 2 & -31 & 0.499 & 0.000141 \\
\hline Inferior Temporal Sulcus & -55 & -38 & -11 & 0.421 & 0.001673 \\
\hline Insula & -35 & 13 & 14 & 0.524 & 0.000056 \\
\hline Intraparietal Sulcus & -27 & -51 & 38 & 0.500 & 0.000137 \\
\hline Lateral Occipitotemporal Gyrus & -36 & 2 & -31 & 0.499 & 0.000141 \\
\hline Lateral Sulcus & -40 & -35 & 19 & 0.466 & 0.000436 \\
\hline Medial Occipitotemporal Gyrus & -12 & -87 & -14 & 0.398 & 0.003203 \\
\hline Medial Prefrontal Cortex & -6 & 36 & 7 & 0.561 & 0.000012 \\
\hline Middle Frontal Gyrus & -26 & 43 & 23 & 0.535 & 0.000037 \\
\hline Middle Occipital Gyrus & -29 & -85 & 1 & 0.392 & 0.003706 \\
\hline Middle Temporal Gyrus & -48 & -26 & -3 & 0.453 & 0.000661 \\
\hline Occipitotemporal Sulcus & -46 & -23 & -20 & 0.501 & 0.000132 \\
\hline Olfactory Sulcus & -9 & 32 & -12 & 0.308 & 0.024758 \\
\hline Orbital Gyri & -21 & 46 & -4 & 0.475 & 0.000321 \\
\hline Orbital Sulci & -19 & 48 & -4 & 0.466 & 0.000436 \\
\hline Parahippocampal Gyrus & -33 & 2 & -33 & 0.346 & 0.01104 \\
\hline Parieto-Occipital Sulcus & -12 & -71 & 47 & 0.416 & 0.001934 \\
\hline Postcentral Gyrus & -43 & -14 & 29 & 0.521 & 0.000063 \\
\hline Postcentral Sulcus & -25 & -39 & 53 & 0.561 & 0.000013 \\
\hline Precentral Gyrus & -31 & -11 & 46 & 0.399 & 0.003081 \\
\hline Precentral Sulcus & -33 & -9 & 46 & 0.592 & 0.000003 \\
\hline Precuneus & -8 & -57 & 29 & 0.435 & 0.001149 \\
\hline Superior Frontal Gyrus & -10 & 63 & 5 & 0.546 & 0.000023 \\
\hline Superior Frontal Sulcus & -19 & 6 & 54 & 0.535 & 0.000037 \\
\hline Superior Occipital Gyrus & -22 & -82 & 23 & 0.675 & 0 \\
\hline Superior Parietal Lobe & -29 & -41 & 49 & 0.529 & 0.000047 \\
\hline Superior Temporal Gyrus & -51 & -36 & 11 & 0.496 & 0.000157 \\
\hline Superior Temporal Sulcus & -48 & -25 & -2 & 0.513 & 0.000085 \\
\hline Supramarginal Gyrus & -52 & -43 & 39 & 0.623 & 0.000001 \\
\hline Transverse Occipital Sulcus & -23 & -77 & 22 & 0.429 & 0.001333 \\
\hline Uncus & -23 & -16 & -21 & 0.209 & 0.132587 \\
\hline
\end{tabular}

Left hemisphere full cortex correlation results on the basis of cortical thickness and total power. 


\begin{tabular}{|c|c|c|c|c|c|}
\hline & TAL X & TAL Y & TAL Z & $\mathbf{r}$ & $\mathbf{p}$ \\
\hline Calcarine Sulcus & -4 & -75 & 15 & -0.632 & 0.000708 \\
\hline Central Sulcus & -34 & -27 & 46 & -0.600 & 0.001525 \\
\hline Cingulate Gyrus & -9 & -45 & 28 & -0.714 & 0.00006 \\
\hline Cingulate Sulcus & -11 & 22 & 28 & -0.736 & 0.000027 \\
\hline Collateral Sulcus & -30 & -34 & -10 & -0.696 & 0.00011 \\
\hline Cuneus & -5 & -77 & 17 & -0.662 & 0.00031 \\
\hline Gyrus Rectus & -6 & 55 & -1 & -0.621 & 0.000921 \\
\hline Inferior Frontal Gyrus & -50 & 18 & 19 & -0.630 & 0.000737 \\
\hline Inferior Frontal Sulcus & -39 & 24 & 25 & -0.633 & 0.000691 \\
\hline Inferior Occipital Gyrus & -28 & -85 & -5 & -0.663 & 0.000308 \\
\hline Inferior Parietal Lobe & -42 & -56 & 39 & -0.685 & 0.000156 \\
\hline Inferior Temporal Gyrus & -39 & 0 & -32 & -0.617 & 0.001029 \\
\hline Inferior Temporal Sulcus & -61 & -27 & -8 & -0.633 & 0.000678 \\
\hline Insula & -32 & 0 & 16 & -0.708 & 0.000075 \\
\hline Intraparietal Sulcus & -26 & -52 & 56 & -0.683 & 0.00017 \\
\hline Lateral Occipitotemporal Gyrus & -40 & -46 & -17 & -0.665 & 0.00029 \\
\hline Lateral Sulcus & -38 & 14 & 12 & -0.668 & 0.00026 \\
\hline Medial Occipitotemporal Gyrus & -11 & -56 & -3 & -0.681 & 0.00018 \\
\hline Medial Prefrontal Cortex & -11 & 42 & 11 & -0.750 & 0.000016 \\
\hline Middle Frontal Gyrus & -43 & 24 & 30 & -0.618 & 0.001004 \\
\hline Middle Occipital Gyrus & -39 & -66 & 14 & -0.636 & 0.00064 \\
\hline Middle Temporal Gyrus & -47 & 1 & -15 & -0.669 & 0.000256 \\
\hline Occipitotemporal Sulcus & -41 & -27 & -17 & -0.610 & 0.001203 \\
\hline Olfactory Sulcus & -7 & 33 & -13 & -0.563 & 0.003379 \\
\hline Orbital Gyri & -18 & 9 & -12 & -0.669 & 0.000253 \\
\hline Orbital Sulci & -16 & 38 & -11 & -0.663 & 0.000304 \\
\hline Parahippocampal Gyrus & -24 & -17 & -21 & -0.575 & 0.002659 \\
\hline Parieto-Occipital Sulcus & -20 & -59 & 13 & -0.601 & 0.001502 \\
\hline Postcentral Gyrus & -49 & -20 & 45 & -0.688 & 0.000145 \\
\hline Postcentral Sulcus & -25 & -43 & 51 & -0.638 & 0.000601 \\
\hline Precentral Gyrus & -7 & -26 & 48 & -0.663 & 0.000302 \\
\hline Precentral Sulcus & -19 & -1 & 57 & 0.707 & 0.000078 \\
\hline Precuneus & -11 & -52 & 31 & -0.697 & 0.000107 \\
\hline Superior Frontal Gyrus & -6 & 37 & 48 & -0.753 & 0.000014 \\
\hline Superior Frontal Sulcus & -13 & 54 & 28 & -0.605 & 0.001341 \\
\hline Superior Occipital Gyrus & -14 & -96 & 9 & -0.645 & 0.0005 \\
\hline Superior Parietal Lobe & -28 & -64 & 43 & 0.641 & 0.000559 \\
\hline Superior Temporal Gyrus & -47 & -9 & -2 & -0.671 & 0.000239 \\
\hline Superior Temporal Sulcus & -52 & -21 & -2 & -0.724 & 0.000044 \\
\hline Supramarginal Gyrus & -52 & -46 & 37 & -0.625 & 0.000838 \\
\hline Transverse Occipital Sulcus & -30 & -72 & 22 & -0.553 & 0.004167 \\
\hline Uncus & -24 & -17 & -21 & -0.575 & 0.002659 \\
\hline
\end{tabular}

Left hemisphere full cortex correlation results on the basis of cortical thickness and total MSNA. 


\begin{tabular}{|c|c|c|c|c|c|}
\hline & TAL X & TAL Y & TAL Z & $\mathbf{r}$ & p \\
\hline Calcarine Sulcus & 15 & -91 & -6 & -0.699 & 0.000101 \\
\hline Central Sulcus & 29 & -28 & 46 & -0.740 & 0.000023 \\
\hline Cingulate Gyrus & 13 & 35 & 18 & -0.759 & 0.000011 \\
\hline Cingulate Sulcus & 12 & 40 & 20 & -0.783 & 0.000004 \\
\hline Collateral Sulcus & 17 & -87 & -15 & -0.841 & 0 \\
\hline Cuneus & 10 & -87 & -1 & -0.743 & 0.000021 \\
\hline Gyrus Rectus & 5 & 24 & -15 & -0.672 & 0.000233 \\
\hline Inferior Frontal Gyrus & 47 & 32 & 6 & -0.839 & 0 \\
\hline Inferior Frontal Sulcus & 40 & 39 & 9 & -0.681 & 0.000176 \\
\hline Inferior Occipital Gyrus & 15 & -88 & -15 & -0.816 & 0.000001 \\
\hline Inferior Parietal Lobe & 46 & -51 & 22 & -0.701 & 0.000094 \\
\hline Inferior Temporal Gyrus & 50 & -11 & -23 & -0.767 & 0.000008 \\
\hline Inferior Temporal Sulcus & 54 & -14 & -19 & -0.709 & 0.000072 \\
\hline Insula & 29 & 17 & 14 & -0.822 & 0 \\
\hline Intraparietal Sulcus & 25 & -62 & 34 & -0.705 & 0.000084 \\
\hline \multicolumn{6}{|l|}{ Lateral Occipitotemporal } \\
\hline Gyrus & 40 & -46 & -15 & -0.747 & 0.000018 \\
\hline Lateral Sulcus & 25 & 13 & -7 & -0.775 & 0.000005 \\
\hline \multicolumn{6}{|l|}{ Medial Occipitotemporal } \\
\hline Gyrus & 14 & -67 & -10 & -0.781 & 0.000004 \\
\hline Medial Prefrontal Cortex & 11 & 50 & 6 & -0.807 & 0.000001 \\
\hline Middle Frontal Gyrus & 37 & 43 & 12 & -0.766 & 0.000008 \\
\hline Middle Occipital Gyrus & 30 & -81 & -3 & -0.707 & 0.000078 \\
\hline Middle Temporal Gyrus & 51 & -51 & 4 & -0.735 & 0.000029 \\
\hline Occipitotemporal Sulcus & 32 & -46 & -14 & -0.727 & 0.000039 \\
\hline Olfactory Sulcus & 14 & 17 & -9 & -0.696 & 0.000111 \\
\hline Orbital Gyri & 26 & 19 & -5 & -0.748 & 0.000017 \\
\hline Orbital Sulci & 32 & 31 & -2 & -0.757 & 0.000012 \\
\hline Parahippocampal Gyrus & 28 & 2 & -34 & -0.733 & 0.000031 \\
\hline Parieto-Occipital Sulcus & 10 & -61 & 52 & -0.798 & 0.000002 \\
\hline Postcentral Gyrus & 18 & -36 & 67 & -0.759 & 0.000011 \\
\hline Postcentral Sulcus & 51 & -19 & 35 & -0.705 & 0.000082 \\
\hline Precentral Gyrus & 48 & -10 & 38 & 0.653 & 0.000398 \\
\hline Precentral Sulcus & 45 & 8 & 26 & -0.705 & 0.000082 \\
\hline Precuneus & 9 & -53 & 29 & -0.760 & 0.000011 \\
\hline Superior Frontal Gyrus & 11 & 50 & 6 & -0.807 & 0.000001 \\
\hline Superior Frontal Sulcus & 23 & 54 & 15 & -0.749 & 0.000017 \\
\hline Superior Occipital Gyrus & 15 & -87 & 15 & -0.763 & 0.000009 \\
\hline Superior Parietal Lobe & 23 & -64 & 33 & -0.723 & 0.000044 \\
\hline Superior Temporal Gyrus & 51 & -18 & 6 & -0.742 & 0.000022 \\
\hline Superior Temporal Sulcus & 43 & -39 & 5 & -0.726 & 0.00004 \\
\hline Supramarginal Gyrus & 50 & -44 & 38 & -0.700 & 0.000098 \\
\hline Transverse Occipital Sulcus & 31 & -74 & 21 & -0.762 & 0.00001 \\
\hline Uncus & 22 & -13 & -18 & -0.520 & 0.00773 \\
\hline
\end{tabular}

Right hemisphere full cortex correlation results on the basis of cortical thickness and burst frequency. 


\begin{tabular}{|c|c|c|c|c|c|}
\hline & TAL X & TAL Y & TAL Z & $\mathbf{r}$ & $\mathbf{p}$ \\
\hline Calcarine Sulcus & 15 & -91 & -6 & -0.720 & 0.00005 \\
\hline Central Sulcus & 29 & -28 & 46 & -0.802 & 0.000001 \\
\hline Cingulate Gyrus & 10 & -5 & 42 & -0.788 & 0.000003 \\
\hline Cingulate Sulcus & 9 & 43 & 22 & -0.786 & 0.000003 \\
\hline Collateral Sulcus & 17 & -87 & -15 & -0.874 & 0 \\
\hline Cuneus & 10 & -87 & -1 & -0.778 & 0.000005 \\
\hline Gyrus Rectus & 5 & 24 & -15 & -0.728 & 0.000036 \\
\hline Inferior Frontal Gyrus & 47 & 32 & 6 & -0.828 & 0 \\
\hline Inferior Frontal Sulcus & 40 & 39 & 9 & -0.699 & 0.000101 \\
\hline Inferior Occipital Gyrus & 15 & -88 & -15 & -0.847 & 0 \\
\hline Inferior Parietal Lobe & 46 & -51 & 21 & -0.674 & 0.000222 \\
\hline Inferior Temporal Gyrus & 50 & -12 & -21 & -0.770 & 0.000007 \\
\hline Inferior Temporal Sulcus & 56 & -33 & -10 & -0.733 & 0.00003 \\
\hline Insula & 29 & 17 & 14 & -0.816 & 0.000001 \\
\hline Intraparietal Sulcus & 25 & -62 & 34 & -0.767 & 0.000008 \\
\hline Lateral Occipitotemporal Gyrus & 40 & -46 & -13 & -0.783 & 0.000004 \\
\hline Lateral Sulcus & 25 & 18 & -7 & -0.761 & 0.00001 \\
\hline Medial Occipitotemporal Gyrus & 10 & -87 & -1 & -0.778 & 0.000005 \\
\hline Medial Prefrontal Cortex & 11 & 50 & 6 & -0.787 & 0.000003 \\
\hline Middle Frontal Gyrus & 37 & 47 & 11 & -0.783 & 0.000004 \\
\hline Middle Occipital Gyrus & 30 & -81 & -3 & -0.714 & 0.000062 \\
\hline Middle Temporal Gyrus & 61 & -37 & -2 & -0.751 & 0.000015 \\
\hline Occipitotemporal Sulcus & 41 & -39 & -17 & -0.760 & 0.00001 \\
\hline Olfactory Sulcus & 14 & 17 & -9 & -0.723 & 0.000044 \\
\hline Orbital Gyri & 23 & 35 & -5 & -0.763 & 0.000009 \\
\hline Orbital Sulci & 32 & 31 & -2 & -0.786 & 0.000003 \\
\hline Parahippocampal Gyrus & 28 & 2 & -34 & -0.715 & 0.000059 \\
\hline Parieto-Occipital Sulcus & 10 & -61 & 52 & -0.856 & 0 \\
\hline Postcentral Gyrus & 20 & -35 & 66 & -0.813 & 0.000001 \\
\hline Postcentral Sulcus & 40 & -31 & 58 & -0.723 & 0.000044 \\
\hline Precentral Gyrus & 30 & -24 & 49 & -0.642 & 0.000542 \\
\hline Precentral Sulcus & 26 & -8 & 48 & -0.742 & 0.000022 \\
\hline Precuneus & 4 & -64 & 29 & -0.717 & 0.000056 \\
\hline Superior Frontal Gyrus & 10 & 53 & 5 & -0.819 & 0.000001 \\
\hline Superior Frontal Sulcus & 23 & 54 & 15 & -0.726 & 0.00004 \\
\hline Superior Occipital Gyrus & 15 & -87 & 15 & -0.763 & 0.000009 \\
\hline Superior Parietal Lobe & 24 & -63 & 33 & -0.751 & 0.000015 \\
\hline Superior Temporal Gyrus & 51 & -16 & 6 & -0.767 & 0.000008 \\
\hline Superior Temporal Sulcus & 43 & -38 & 7 & -0.768 & 0.000007 \\
\hline Supramarginal Gyrus & 40 & -42 & 38 & -0.761 & 0.00001 \\
\hline Transverse Occipital Sulcus & 31 & -74 & 21 & -0.780 & 0.000004 \\
\hline Uncus & 22 & -13 & -18 & -0.541 & 0.005272 \\
\hline
\end{tabular}

Right hemisphere full cortex correlation results on the basis of cortical thickness and burst incidence. 


\begin{tabular}{|c|c|c|c|c|c|}
\hline & TAL $\mathbf{X}$ & TAL Y & TAL Z & $\mathbf{r}$ & p \\
\hline Calcarine Sulcus & 16 & -68 & 6 & 0.420 & 0.001404 \\
\hline Central Sulcus & 24 & -26 & 61 & 0.459 & 0.000425 \\
\hline Cingulate Gyrus & 4 & 9 & 31 & 0.454 & 0.000496 \\
\hline Cingulate Sulcus & 10 & 47 & 19 & 0.423 & 0.001279 \\
\hline Collateral Sulcus & 35 & -13 & -25 & 0.457 & 0.000453 \\
\hline Cuneus & 9 & -89 & 10 & 0.441 & 0.000757 \\
\hline Gyrus Rectus & 6 & 33 & -10 & 0.444 & 0.000684 \\
\hline Inferior Frontal Gyrus & 38 & 40 & 9 & 0.508 & 0.000074 \\
\hline Inferior Frontal Sulcus & 39 & 24 & 22 & 0.492 & 0.000137 \\
\hline Inferior Occipital Gyrus & 20 & -95 & -5 & 0.384 & 0.003785 \\
\hline Inferior Parietal Lobe & 43 & -63 & 34 & 0.496 & 0.000116 \\
\hline Inferior Temporal Gyrus & 46 & -5 & -29 & 0.510 & 0.000069 \\
\hline Inferior Temporal Sulcus & 56 & -18 & -15 & 0.445 & 0.000674 \\
\hline Insula & 31 & -21 & 17 & 0.550 & 0.000013 \\
\hline Intraparietal Sulcus & 23 & -61 & 54 & 0.415 & 0.001641 \\
\hline Lateral Occipitotemporal Gyrus & 36 & -10 & -26 & 0.539 & 0.000022 \\
\hline Lateral Sulcus & 23 & 15 & -10 & 0.597 & 0.000002 \\
\hline Medial Occipitotemporal Gyrus & 8 & -62 & -2 & 0.487 & 0.000164 \\
\hline Medial Prefrontal Cortex & 10 & 49 & 3 & 0.442 & 0.000733 \\
\hline Middle Frontal Gyrus & 25 & 49 & 6 & 0.566 & 0.000007 \\
\hline Middle Occipital Gyrus & 44 & -71 & 3 & 0.456 & 0.000463 \\
\hline Middle Temporal Gyrus & 52 & -7 & -16 & 0.446 & 0.000645 \\
\hline Occipitotemporal Sulcus & 38 & -9 & -25 & 0.432 & 0.000999 \\
\hline Olfactory Sulcus & 12 & 28 & -10 & 0.430 & 0.001044 \\
\hline Orbital Gyri & 20 & 34 & -8 & 0.511 & 0.000068 \\
\hline Orbital Sulci & 29 & 36 & -1 & 0.550 & 0.000014 \\
\hline Parahippocampal Gyrus & 29 & -9 & -23 & 0.486 & 0.000171 \\
\hline Parieto-Occipital Sulcus & 20 & -63 & 20 & 0.688 & 0 \\
\hline Postcentral Gyrus & 36 & -33 & 46 & 0.551 & 0.000013 \\
\hline Postcentral Sulcus & 48 & -23 & 35 & 0.515 & 0.000058 \\
\hline Precentral Gyrus & 25 & -26 & 62 & 0.471 & 0.00028 \\
\hline Precentral Sulcus & 35 & 4 & 29 & 0.450 & 0.000563 \\
\hline Precuneus & 6 & -70 & 39 & 0.479 & 0.000214 \\
\hline Superior Frontal Gyrus & 19 & 42 & 35 & 0.602 & 0.000001 \\
\hline Superior Frontal Sulcus & 21 & 28 & 39 & 0.492 & 0.000137 \\
\hline Superior Occipital Gyrus & 8 & -95 & 3 & 0.373 & 0.005075 \\
\hline Superior Parietal Lobe & 30 & -48 & 51 & 0.447 & 0.000626 \\
\hline Superior Temporal Gyrus & 31 & -21 & 17 & 0.550 & 0.000013 \\
\hline Superior Temporal Sulcus & 45 & -13 & -11 & 0.502 & 0.000095 \\
\hline Supramarginal Gyrus & 57 & -15 & 18 & 0.527 & 0.000036 \\
\hline Transverse Occipital Sulcus & 25 & -70 & 29 & 0.486 & 0.000168 \\
\hline Uncus & 23 & -12 & -20 & 0.350 & 0.008742 \\
\hline
\end{tabular}

Right hemisphere full cortex correlation results on the basis of cortical thickness and baroreflex sensitivity. 


\begin{tabular}{|c|c|c|c|c|c|}
\hline & TAL X & TAL Y & TAL Z & $\mathbf{r}$ & $\mathbf{p}$ \\
\hline Calcarine Sulcus & 17 & -52 & 8 & 0.461 & 0.000518 \\
\hline Central Sulcus & 54 & -6 & 36 & 0.406 & 0.002546 \\
\hline Cingulate Gyrus & 10 & 36 & 12 & $\begin{array}{c}0.488 \\
-\end{array}$ & 0.000211 \\
\hline Cingulate Sulcus & 9 & -46 & 60 & 0.459 & 0.000553 \\
\hline Collateral Sulcus & 17 & -86 & -14 & 0.447 & 0.000795 \\
\hline Cuneus & 9 & -89 & 10 & 0.428 & 0.001406 \\
\hline Gyrus Rectus & 6 & 51 & -7 & 0.372 & 0.006054 \\
\hline Inferior Frontal Gyrus & 47 & 9 & 23 & 0.515 & 0.000079 \\
\hline Inferior Frontal Sulcus & 39 & 23 & 23 & 0.423 & 0.001602 \\
\hline Inferior Occipital Gyrus & 15 & -85 & -12 & 0.442 & 0.000933 \\
\hline Inferior Parietal Lobe & 38 & -59 & 36 & 0.455 & 0.000625 \\
\hline Inferior Temporal Gyrus & 53 & -20 & -19 & 0.412 & 0.002149 \\
\hline Inferior Temporal Sulcus & 58 & -26 & -12 & 0.444 & 0.000855 \\
\hline Insula & 31 & 13 & 7 & 0.560 & 0.000013 \\
\hline $\begin{array}{l}\text { Intraparietal Sulcus } \\
\text { Lateral Occipitotemporal }\end{array}$ & 35 & -56 & 36 & 0.518 & 0.000071 \\
\hline Gyrus & 36 & -9 & -26 & 0.521 & 0.000064 \\
\hline $\begin{array}{l}\text { Lateral Sulcus } \\
\text { Medial Occipitotemporal }\end{array}$ & 39 & -23 & 20 & 0.489 & 0.000202 \\
\hline Gyrus & 20 & -65 & -8 & 0.427 & 0.001442 \\
\hline Medial Prefrontal Cortex & 10 & 48 & 1 & 0.562 & 0.000012 \\
\hline Middle Frontal Gyrus & 26 & 49 & 5 & 0.515 & 0.00008 \\
\hline Middle Occipital Gyrus & 44 & -71 & 3 & 0.372 & 0.006074 \\
\hline Middle Temporal Gyrus & 54 & -47 & 6 & 0.443 & 0.000884 \\
\hline Occipitotemporal Sulcus & 37 & -9 & -26 & 0.516 & 0.000076 \\
\hline Olfactory Sulcus & 13 & 28 & -10 & 0.486 & 0.000224 \\
\hline Orbital Gyri & 14 & 28 & -12 & 0.548 & 0.000022 \\
\hline Orbital Sulci & 14 & 52 & -8 & 0.470 & 0.000379 \\
\hline Parahippocampal Gyrus & 30 & -1 & -34 & 0.314 & 0.021909 \\
\hline Parieto-Occipital Sulcus & 20 & -63 & 20 & 0.514 & 0.000081 \\
\hline Postcentral Gyrus & 42 & -27 & 38 & 0.551 & 0.000019 \\
\hline Postcentral Sulcus & 41 & -36 & 39 & 0.509 & 0.000099 \\
\hline Precentral Gyrus & 8 & -25 & 70 & 0.385 & 0.004462 \\
\hline Precentral Sulcus & 20 & -5 & 59 & 0.459 & 0.000542 \\
\hline Precuneus & 12 & -49 & 33 & 0.449 & 0.000743 \\
\hline Superior Frontal Gyrus & 10 & 48 & 1 & 0.562 & 0.000012 \\
\hline Superior Frontal Sulcus & 25 & 50 & 27 & 0.463 & 0.00048 \\
\hline Superior Occipital Gyrus & 12 & -89 & 11 & 0.511 & 0.000092 \\
\hline Superior Parietal Lobe & 26 & -71 & 21 & 0.404 & 0.002723 \\
\hline Superior Temporal Gyrus & 61 & -31 & 12 & 0.472 & 0.000365 \\
\hline Superior Temporal Sulcus & 45 & -14 & -9 & 0.439 & 0.000999 \\
\hline Supramarginal Gyrus & 55 & -42 & 29 & 0.526 & 0.000053 \\
\hline Transverse Occipital Sulcus & 29 & -70 & 19 & 0.442 & 0.000917 \\
\hline Uncus & 24 & -19 & -19 & 0.333 & 0.014862 \\
\hline
\end{tabular}

Right hemisphere full cortex correlation results on the basis of cortical thickness and In-high frequency. 


\begin{tabular}{|c|c|c|c|c|c|}
\hline & TAL X & TAL Y & TAL Z & $\mathbf{r}$ & $\mathbf{p}$ \\
\hline Calcarine Sulcus & 12 & -98 & -3 & 0.440 & 0.000983 \\
\hline Central Sulcus & 54 & -6 & 36 & 0.416 & 0.001933 \\
\hline Cingulate Gyrus & 8 & 35 & 14 & 0.512 & 0.000088 \\
\hline Cingulate Sulcus & 8 & 50 & 28 & 0.423 & 0.001613 \\
\hline Collateral Sulcus & 34 & -9 & -27 & 0.523 & 0.000058 \\
\hline Cuneus & 21 & -82 & 33 & 0.445 & 0.000841 \\
\hline Gyrus Rectus & 6 & 39 & -15 & 0.387 & 0.004247 \\
\hline Inferior Frontal Gyrus & 27 & 53 & 1 & 0.415 & 0.001994 \\
\hline Inferior Frontal Sulcus & 45 & 35 & 6 & 0.388 & 0.004115 \\
\hline Inferior Occipital Gyrus & 45 & -59 & -7 & 0.354 & 0.009221 \\
\hline Inferior Parietal Lobe & 42 & -64 & 32 & 0.548 & 0.000022 \\
\hline Inferior Temporal Gyrus & 31 & 2 & -33 & 0.404 & 0.002731 \\
\hline Inferior Temporal Sulcus & 57 & -22 & -13 & 0.444 & 0.000876 \\
\hline Insula & 37 & 16 & 14 & 0.582 & 0.000005 \\
\hline Intraparietal Sulcus & 47 & -53 & 33 & 0.479 & 0.000286 \\
\hline \multicolumn{6}{|l|}{ Lateral Occipitotemporal } \\
\hline Gyrus & 35 & -9 & -27 & 0.507 & 0.000107 \\
\hline Lateral Sulcus & 38 & 14 & 13 & 0.643 & 0 \\
\hline \multicolumn{6}{|l|}{ Medial Occipitotemporal } \\
\hline Gyrus & 21 & -67 & -8 & 0.458 & 0.000566 \\
\hline Medial Prefrontal Cortex & 10 & 48 & 1 & 0.549 & 0.000021 \\
\hline Middle Frontal Gyrus & 32 & 1 & 55 & 0.494 & 0.000171 \\
\hline Middle Occipital Gyrus & 29 & -83 & -2 & 0.290 & 0.034953 \\
\hline Middle Temporal Gyrus & 54 & -47 & 6 & 0.474 & 0.000337 \\
\hline Occipitotemporal Sulcus & 37 & -9 & -26 & 0.492 & 0.000184 \\
\hline Olfactory Sulcus & 13 & 29 & -11 & 0.405 & 0.002636 \\
\hline Orbital Gyri & 14 & 27 & -12 & 0.459 & 0.000551 \\
\hline Orbital Sulci & 29 & 36 & -1 & 0.393 & 0.00363 \\
\hline Parahippocampal Gyrus & 31 & -9 & -27 & 0.371 & 0.006203 \\
\hline Parieto-Occipital Sulcus & 11 & -60 & 53 & 0.455 & 0.000613 \\
\hline Postcentral Gyrus & 42 & -28 & 38 & 0.512 & 0.00009 \\
\hline Postcentral Sulcus & 41 & -38 & 39 & 0.526 & 0.000053 \\
\hline Precentral Gyrus & 53 & -5 & 37 & 0.504 & 0.000121 \\
\hline Precentral Sulcus & 44 & 2 & 23 & 0.454 & 0.000645 \\
\hline Precuneus & 8 & -60 & 20 & 0.348 & 0.010699 \\
\hline Superior Frontal Gyrus & 15 & 9 & 57 & 0.581 & 0.000005 \\
\hline Superior Frontal Sulcus & 31 & 1 & 55 & 0.496 & 0.000158 \\
\hline Superior Occipital Gyrus & 24 & -85 & 12 & 0.412 & 0.002169 \\
\hline Superior Parietal Lobe & 9 & -58 & 54 & 0.479 & 0.000289 \\
\hline Superior Temporal Gyrus & 61 & -30 & 11 & 0.588 & 0.000004 \\
\hline Superior Temporal Sulcus & 49 & 2 & -12 & 0.420 & 0.001751 \\
\hline Supramarginal Gyrus & 55 & -42 & 29 & 0.631 & 0 \\
\hline Transverse Occipital Sulcus & 29 & -70 & 19 & 0.348 & 0.010561 \\
\hline Uncus & 22 & -13 & -18 & 0.307 & 0.025303 \\
\hline
\end{tabular}

Right hemisphere full cortex correlation results on the basis of cortical thickness and SD1. 


\begin{tabular}{|c|c|c|c|c|c|}
\hline & TAL X & TAL Y & TAL Z & $\mathbf{r}$ & $\mathbf{p}$ \\
\hline Calcarine Sulcus & 12 & -98 & -3 & 0.453 & 0.000665 \\
\hline Central Sulcus & 36 & -22 & 41 & 0.408 & 0.002393 \\
\hline Cingulate Gyrus & 10 & 36 & 12 & 0.502 & 0.000126 \\
\hline Cingulate Sulcus & 11 & -39 & 49 & 0.456 & 0.000596 \\
\hline Collateral Sulcus & 29 & -32 & -18 & 0.435 & 0.001145 \\
\hline Cuneus & 23 & -78 & 33 & 0.469 & 0.000401 \\
\hline Gyrus Rectus & 6 & 51 & -7 & 0.471 & 0.000369 \\
\hline Inferior Frontal Gyrus & 45 & 11 & 11 & 0.464 & 0.000468 \\
\hline Inferior Frontal Sulcus & 39 & 23 & 23 & 0.387 & 0.00422 \\
\hline Inferior Occipital Gyrus & 15 & -85 & -12 & 0.420 & 0.001723 \\
\hline Inferior Parietal Lobe & 42 & -62 & 35 & 0.506 & 0.000109 \\
\hline Inferior Temporal Gyrus & 44 & -40 & -14 & 0.460 & 0.000527 \\
\hline Inferior Temporal Sulcus & 53 & -21 & -16 & 0.473 & 0.000342 \\
\hline Insula & 34 & -17 & 21 & 0.542 & 0.000028 \\
\hline Intraparietal Sulcus & 29 & -55 & 48 & 0.448 & 0.000763 \\
\hline Lateral Occipitotemporal Gyrus & 41 & -41 & -14 & 0.500 & 0.000135 \\
\hline Lateral Sulcus & 44 & 11 & 10 & 0.488 & 0.000208 \\
\hline Medial Occipitotemporal Gyrus & 18 & -66 & -9 & 0.451 & 0.000704 \\
\hline Medial Prefrontal Cortex & 10 & 49 & 3 & 0.607 & 0.000001 \\
\hline Middle Frontal Gyrus & 24 & 50 & 7 & 0.493 & 0.000178 \\
\hline Middle Occipital Gyrus & 20 & -95 & -5 & 0.364 & 0.007345 \\
\hline Middle Temporal Gyrus & 45 & -15 & -10 & 0.462 & 0.0005 \\
\hline Occipitotemporal Sulcus & 34 & -54 & -14 & 0.450 & 0.000729 \\
\hline Olfactory Sulcus & 13 & 29 & -11 & 0.467 & 0.000422 \\
\hline Orbital Gyri & 14 & 28 & -12 & 0.535 & 0.000037 \\
\hline Orbital Sulci & 14 & 50 & -9 & 0.449 & 0.000745 \\
\hline Parahippocampal Gyrus & 29 & -10 & -23 & 0.396 & 0.003348 \\
\hline Parieto-Occipital Sulcus & 12 & -60 & 53 & 0.518 & 0.000072 \\
\hline Postcentral Gyrus & 42 & -26 & 39 & 0.542 & 0.000028 \\
\hline Postcentral Sulcus & 41 & -36 & 39 & 0.485 & 0.000232 \\
\hline Precentral Gyrus & 8 & -25 & 70 & 0.449 & 0.000754 \\
\hline Precentral Sulcus & 46 & 8 & 24 & 0.431 & 0.001269 \\
\hline Precuneus & 12 & -48 & 33 & 0.441 & 0.00096 \\
\hline Superior Frontal Gyrus & 10 & 49 & 3 & 0.607 & 0.000001 \\
\hline Superior Frontal Sulcus & 22 & 28 & 38 & 0.413 & 0.002098 \\
\hline Superior Occipital Gyrus & 26 & -87 & 9 & 0.467 & 0.000416 \\
\hline Superior Parietal Lobe & 26 & -71 & 21 & 0.452 & 0.00068 \\
\hline Superior Temporal Gyrus & 48 & 4 & -7 & 0.458 & 0.000564 \\
\hline Superior Temporal Sulcus & 45 & -14 & -9 & 0.474 & 0.000332 \\
\hline Supramarginal Gyrus & 56 & -27 & 30 & 0.522 & 0.00006 \\
\hline Transverse Occipital Sulcus & 27 & -70 & 20 & 0.491 & 0.000192 \\
\hline Uncus & 23 & -12 & -20 & 0.304 & 0.027101 \\
\hline
\end{tabular}

Right hemisphere full cortex correlation results on the basis of cortical thickness and SDNN. 


\begin{tabular}{|c|c|c|c|c|c|}
\hline & TAL X & TAL Y & TAL Z & $\mathbf{r}$ & $\mathbf{p}$ \\
\hline Calcarine Sulcus & 12 & -98 & -3 & 0.490 & 0.000194 \\
\hline Central Sulcus & 35 & -21 & 39 & 0.462 & 0.000502 \\
\hline Cingulate Gyrus & 7 & -38 & 38 & 0.473 & 0.000351 \\
\hline Cingulate Sulcus & 11 & -39 & 49 & 0.481 & 0.000267 \\
\hline Collateral Sulcus & 29 & -32 & -18 & 0.469 & 0.000401 \\
\hline Cuneus & 23 & -77 & 34 & 0.466 & 0.000438 \\
\hline Gyrus Rectus & 6 & 51 & -7 & 0.472 & 0.000357 \\
\hline Inferior Frontal Gyrus & 43 & 22 & 20 & 0.445 & 0.00084 \\
\hline Inferior Frontal Sulcus & 39 & 25 & 23 & 0.456 & 0.000608 \\
\hline Inferior Occipital Gyrus & 15 & -85 & -12 & 0.450 & 0.000722 \\
\hline Inferior Parietal Lobe & 43 & -63 & 34 & 0.597 & 0.000002 \\
\hline Inferior Temporal Gyrus & 44 & -40 & -14 & 0.489 & 0.000202 \\
\hline Inferior Temporal Sulcus & 54 & -21 & -14 & 0.559 & 0.000013 \\
\hline Insula & 34 & -17 & 21 & 0.607 & 0.000001 \\
\hline Intraparietal Sulcus & 47 & -53 & 33 & 0.468 & 0.000406 \\
\hline Lateral Occipitotemporal Gyrus & 41 & -40 & -15 & 0.552 & 0.000018 \\
\hline Lateral Sulcus & 38 & 14 & 13 & 0.561 & 0.000012 \\
\hline Medial Occipitotemporal Gyrus & 18 & -66 & -9 & 0.503 & 0.000123 \\
\hline Medial Prefrontal Cortex & 10 & 49 & 3 & 0.591 & 0.000003 \\
\hline Middle Frontal Gyrus & 24 & 50 & 7 & 0.527 & 0.00005 \\
\hline Middle Occipital Gyrus & 30 & -82 & -1 & 0.383 & 0.004669 \\
\hline Middle Temporal Gyrus & 45 & -15 & -10 & 0.528 & 0.000049 \\
\hline Occipitotemporal Sulcus & 37 & -9 & -26 & 0.472 & 0.000365 \\
\hline Olfactory Sulcus & 13 & 29 & -11 & 0.521 & 0.000064 \\
\hline Orbital Gyri & 14 & 28 & -12 & 0.568 & 0.000009 \\
\hline Orbital Sulci & 38 & 37 & -4 & 0.477 & 0.000301 \\
\hline Parahippocampal Gyrus & 29 & -9 & -23 & 0.396 & 0.003361 \\
\hline Parieto-Occipital Sulcus & 12 & -60 & 53 & 0.538 & 0.000033 \\
\hline Postcentral Gyrus & 42 & -28 & 38 & 0.540 & 0.00003 \\
\hline Postcentral Sulcus & 42 & -35 & 39 & 0.484 & 0.000244 \\
\hline Precentral Gyrus & 8 & -25 & 70 & 0.451 & 0.000711 \\
\hline Precentral Sulcus & 24 & -4 & 50 & 0.424 & 0.001538 \\
\hline Precuneus & 11 & -48 & 35 & 0.499 & 0.000143 \\
\hline Superior Frontal Gyrus & 10 & 49 & 3 & 0.591 & 0.000003 \\
\hline Superior Frontal Sulcus & 22 & 28 & 38 & 0.490 & 0.000195 \\
\hline Superior Occipital Gyrus & 24 & -89 & 11 & 0.474 & 0.000339 \\
\hline Superior Parietal Lobe & 17 & -65 & 43 & 0.506 & 0.000112 \\
\hline Superior Temporal Gyrus & 53 & -18 & 7 & 0.465 & 0.000448 \\
\hline Superior Temporal Sulcus & 45 & -14 & -9 & 0.527 & 0.000051 \\
\hline Supramarginal Gyrus & 55 & -42 & 29 & 0.576 & 0.000006 \\
\hline Transverse Occipital Sulcus & 23 & -77 & 34 & 0.466 & 0.000438 \\
\hline Uncus & 23 & -12 & -20 & 0.304 & 0.026957 \\
\hline
\end{tabular}

Right hemisphere full cortex correlation results on the basis of cortical thickness and total power. 


\begin{tabular}{|c|c|c|c|c|c|}
\hline & TAL X & TAL Y & TAL Z & $\mathbf{r}$ & $\mathbf{p}$ \\
\hline Calcarine Sulcus & 11 & -98 & 0 & -0.649 & 0.000454 \\
\hline Central Sulcus & 30 & -28 & 46 & -0.689 & 0.000141 \\
\hline Cingulate Gyrus & 5 & -45 & 10 & -0.709 & 0.000072 \\
\hline Cingulate Sulcus & 9 & 43 & 30 & -0.768 & 0.000007 \\
\hline Collateral Sulcus & 30 & -44 & -9 & -0.760 & 0.000011 \\
\hline Cuneus & 23 & -79 & 32 & -0.728 & 0.000038 \\
\hline Gyrus Rectus & 6 & 26 & -16 & -0.650 & 0.00044 \\
\hline Inferior Frontal Gyrus & 49 & 30 & 4 & -0.792 & 0.000002 \\
\hline Inferior Frontal Sulcus & 46 & 34 & 4 & -0.674 & 0.000221 \\
\hline Inferior Occipital Gyrus & 15 & -88 & -15 & -0.729 & 0.000036 \\
\hline Inferior Parietal Lobe & 42 & -65 & 19 & 0.651 & 0.00042 \\
\hline Inferior Temporal Gyrus & 49 & -8 & -22 & -0.664 & 0.000294 \\
\hline Inferior Temporal Sulcus & 54 & -14 & -19 & -0.627 & 0.000798 \\
\hline Insula & 25 & 17 & -6 & -0.733 & 0.000031 \\
\hline Intraparietal Sulcus & 25 & -62 & 34 & -0.615 & 0.001073 \\
\hline Lateral Occipitotemporal Gyrus & 28 & 2 & -34 & -0.749 & 0.000017 \\
\hline Lateral Sulcus & 25 & 17 & -7 & -0.750 & 0.000016 \\
\hline Medial Occipitotemporal Gyrus & 14 & -67 & -10 & -0.685 & 0.000158 \\
\hline Medial Prefrontal Cortex & 11 & 50 & 6 & -0.743 & 0.000021 \\
\hline Middle Frontal Gyrus & 37 & 47 & 11 & -0.750 & 0.000016 \\
\hline Middle Occipital Gyrus & 30 & -81 & -3 & -0.610 & 0.001199 \\
\hline Middle Temporal Gyrus & 51 & -51 & 4 & -0.636 & 0.000634 \\
\hline Occipitotemporal Sulcus & 33 & -47 & -14 & -0.657 & 0.000362 \\
\hline Olfactory Sulcus & 13 & 26 & -9 & -0.622 & 0.0009 \\
\hline Orbital Gyri & 25 & 17 & -6 & -0.733 & 0.000031 \\
\hline Orbital Sulci & 37 & 44 & -4 & -0.688 & 0.000145 \\
\hline Parahippocampal Gyrus & 28 & 2 & -34 & -0.749 & 0.000017 \\
\hline Parieto-Occipital Sulcus & 10 & -61 & 52 & -0.726 & 0.00004 \\
\hline Postcentral Gyrus & 29 & -34 & 62 & -0.767 & 0.000008 \\
\hline Postcentral Sulcus & 40 & -31 & 58 & -0.628 & 0.00077 \\
\hline Precentral Gyrus & 5 & -22 & 52 & -0.582 & 0.002293 \\
\hline Precentral Sulcus & 18 & -13 & 58 & -0.618 & 0.000997 \\
\hline Precuneus & 8 & -55 & 30 & -0.710 & 0.000069 \\
\hline Superior Frontal Gyrus & 10 & 52 & 6 & -0.768 & 0.000008 \\
\hline Superior Frontal Sulcus & 31 & 9 & 52 & -0.741 & 0.000023 \\
\hline Superior Occipital Gyrus & 24 & -79 & 30 & -0.692 & 0.000128 \\
\hline Superior Parietal Lobe & 23 & -64 & 33 & -0.649 & 0.000443 \\
\hline Superior Temporal Gyrus & 51 & -18 & 6 & -0.674 & 0.000223 \\
\hline Superior Temporal Sulcus & 48 & -43 & 6 & -0.690 & 0.000137 \\
\hline Supramarginal Gyrus & 40 & -42 & 38 & -0.717 & 0.000054 \\
\hline Transverse Occipital Sulcus & 31 & -74 & 21 & -0.671 & 0.000242 \\
\hline Uncus & 22 & -13 & -18 & -0.529 & 0.006551 \\
\hline
\end{tabular}

Right hemisphere full cortex correlation results on the basis of cortical thickness and total MSNA. 


\section{Appendix 2 Ethics Approval}

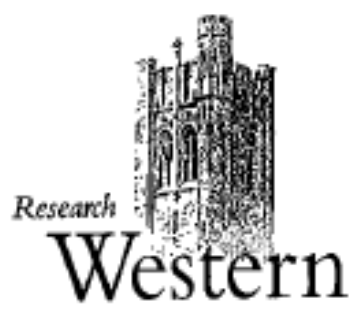

Use of Human Participants - Ethics Approval Notice

Principal Investigator: Dr. Kevin Shoemaker

Review Number: 17810

Review Level: Delegated

Approved Local Adult Participants: 200

Approved Local Minor Participants: 0

Protocol Title: Architecture of Cortical Somatosensory and Autonomic Neural Networks Supporting Muscular

Movement: Impacts of Vascular Disease and Exercise Interventions.

Department \& Institution: Kinesiology, University of Western Ontario

Sponsor: Canadian Institutes of Health Research

Ethics Approval Date: August 12, 2011

Expiry Date: December 31, 2016

Documents Roviewed \& Approved \& Documents Received for Information:

\begin{tabular}{|l|l|l|}
\hline Document Name & Comments & Version Date \\
\hline Revised Letter of Information \& Consent & & \\
\hline Other & Revised recruitment letter & \\
\hline
\end{tabular}

This is to notify you that The University of Western Ontario Research Ethies Board for Health Sciences Research Involving Hurnan Subjects (HSREB) which is organized and operates according to the Tri-Council Policy Statement: Ethical Conduct of Researeh Involving Hamans and the Health Canada/CH Good Clinical Practice Practices: Consolidated Guidelines; and the applicable laws and regulations of Ontario has reviewed and granted aporoval to the above referenced revision(s) or

amesdment(s) on the approval date noted above. The membership of this REB also complies with the membership requirements for REB's as defined in Division 5 of the Food and Drug Regalations.

The ethics approval for this study shall remain valid until the expiry date noted above assuming timely and acceptable responses to the HSREB's periodic requests for surveillance and monitoring information, If you require an updated approval notice prior to thet time you must request it using the UWO Updated Approval Request Form.

Members of the HSREB who are named as investigators in research studies, or deciare a conflict of interest, do not participate in discession related to, nor vote on, such studies when they aro presented to the HSREB.

The Chair of the HSREB is Dr. Joseph Gilbert. The UWO HSREB is registered with the U.S. Department of Health \& Human Services under the IRB registration number IRB 00000940.

\begin{tabular}{|c|c|c|}
\hline \multicolumn{3}{|c|}{ Eutics ORacer to Cuatact for Further Information } \\
\hline 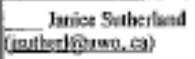 & 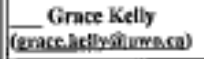 & 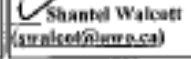 \\
\hline
\end{tabular}




\title{
Curriculum Vitae
}

\author{
EDUCATION AND TRAINING: \\ Jan 2013 - Present PhD Candidate \\ Department of Kinesiology, Integrative Physiology, Neurovascular \\ Research Laboratory \\ The University of Western Ontario, London, ON \\ Thesis: Neuroprotective Effect of Exercise \\ Supervisor: Dr. J Kevin Shoemaker \\ Sept 2008 - 08/2010 Masters of Science \\ Department of Kinesiology, Integrative Physiology, Neurovascular \\ Research Laboratory \\ The University of Western Ontario, London, ON \\ Thesis: Forebrain Cortical Alterations with Hypertension \\ Supervisor: Dr. J Kevin Shoemaker \\ September $2009 \quad$ Invited fMRI Visiting Fellowship \\ Martinos Center for Biomedical Imaging; Boston, Massachusetts \\ Sept $2004-05 / 2008$ \\ Bachelor of Arts; Honors Specialization \\ Department of Kinesiology, Faculty of Health Sciences \\ The University of Western Ontario, London, ON
}

\section{ACADEMIC AWARDS AND ACCOLADES:}

$\begin{array}{ll}2016 & \text { Recognition of Achievement: Outstanding Contribution to Teaching } \\ 2015 & \text { CSEP Oral Presentation Graduate Award Finalist } \\ 2015 & \text { American Autonomic Society Travel Fellowship } \\ 2015 & \text { Advanced Teaching Program } \\ 2015 & \text { Certificate in University Teaching and Learning } \\ 2014 & \text { 3-Minute-Thesis Competition Finalist } \\ 2009 & \text { NSERC Alexander Graham Bell Scholarship - CGS M } \\ 2008 & \text { NSERC Undergraduate Student Research Award } \\ 2008 & \text { The University of Western Ontario Dean's Honor List } \\ 2004 & \text { The University of Western Ontario Scholarship of Distinction }\end{array}$

Value

Certificate

$\$ 500.00$

$\$ 2,000.00$

Certificate

Certificate

University-level

$\$ 17,500.00$

$\$ 4,500.00$

Average $>85 \%$

$\$ 2,500.00$

\section{EXPERIENCE:}

Research Experience

09/2010 - 12/2012

$01 / 2008-09 / 2008$

$09 / 2006-04 / 2008$

Teaching Experience

09/2009 - Present

$02 / 2016-04 / 2016$

\section{Neuroimaging Research Assistant}

Neurovascular Research Laboratory, Department of Kinesiology

The University of Western Ontario, London, ON

Research Assistant

Neurovascular Research Laboratory, Department of Kinesiology

The University of Western Ontario, London, ON

Research Assistant

Exercise Nutrition Laboratory, Department of Kinesiology

The University of Western Ontario, London, ON

Wellness Instructor

IVEY Spencer Leadership Centre, London, ON

Part-Time Faculty, Yoga Instructor 


$\begin{array}{ll}09 / 2013-04 / 2015 & \begin{array}{l}\text { Department of Kinesiology, Western University, London ON } \\ \text { Graduate Bioscience Coordinator } \\ \text { Kinesiology Graduate Students } \\ \text { The University of Western Ontario, London, ON } \\ \text { Invited Guest Lecture, Exercise Physiology }\end{array} \\ 04 / 2014-04 / 2014 & \begin{array}{l}\text { Fanshawe College, London, ON } \\ \text { Teachers Assistant: Clinical Anatomy } \\ \text { The University of Western Ontario, London, ON } \\ \text { Anatomy Instruction for Yoga Teacher Training } \\ \text { Osoyoga Inc, London, ON } \\ \text { Teachers Assistant: Clinical Anatomy } \\ \text { The University of Western Ontario, London, ON } \\ \text { Senior Fitness Instructor } \\ \text { Retired Researchers Association, London, ON }\end{array} \\ 09 / 2009-06 / 2013-05 / 2010-05 / 2010 & \end{array}$

Academic and Administrative Experience

May 2014

April 2014

$09 / 2009-05 / 2010$

$09 / 2009-05 / 2010$

$10 / 2008-08 / 2009$

$01 / 2006-05 / 2007$

$09 / 2005-05 / 2006$

GradCast - CHRW Graduate Student Research Radio; UWO, London, ON Participation on a hiring committee as a graduate student; UWO, London, ON Graduate Students Health Plan Chairperson; UWO, London, ON Levy Funds Masters Representative; Dept. of Kinesiology, UWO, London, ON Graduate Student Health Plan Committee Member; UWO, London, ON Undergraduate Teachers Assistant; Dept. of Anatomy, UWO, London, ON Physiotherapist Assistant; Fowler Kennedy Sports Medicine Clinic; London, ON

RESEARCH PUBLICATIONS AND ACTIVITIES:

Articles Published or Accepted in Refereed Journals

1. KN Wood, R Nikolov, JK Shoemaker. (2016) Impact of Long-Term Endurance Training Versus Guideline-Based Physical Activity on Brain Structure in Healthy Aging. Frontiers in Aging Neuroscience; 8.

2. KN Norton, MB Badrov, CC Barron, N Suskin, A Heinecke, JK Shoemaker. (2015) Coronary Artery Disease Affects Cortical Circuitry Associated with Brain-Heart Integration during Volitional Exercise. J Neurophysiol. 114(2):835-45.

3. JK Shoemaker, KN Norton, J Baker, T Luchyshyn. (2015) Forebrain organization for autonomic cardiovascular control. Invited Review: Autonomic Neuroscience, Basic and Clinical;188:5-9.

4. KN Norton, TA Luchyshyn, JK Shoemaker. (2013) Evidence for a medial prefrontal cortex - hippocampal axis associated with heart rate control in conscious humans. Brain Research;1538:104-15.

5. AM Kiviniemi, S Tiinanen, AJ Hautala, T Seppänen, KN Norton, MF Frances, RP Nolan, HV Huikuri, MP Tulppo, JK Shoemaker. (2010) Low-frequency oscillations in R$\mathrm{R}$ interval and blood pressure across the continuum of cardiovascular risk. Auton Neurosci.; 158(1-2):92-9.

6. M Zamir, KN Norton, A Fleischhauer, MF Frances, R Goswami, CW Usselman, RP Nolan, JK Shoemaker. (2009) Dynamic responsiveness of the vascular bed as a regulatory mechanism in vasomotor control. J Gen Physiol;134(1):69-75.

Papers in Submission

1. KN Wood, MB Badrov, MR Speechley, JK Shoemaker. (2016) Regional cerebral cortical thickness correlates with autonomic outflow. Submitted to Brain Structure Function July 18, 2016: BSAF-D-16-00378. 
2. KN Wood, TA Luchyshyn, JK Shoemaker. (2016) High cardiorespiratory fitness in middle-age preserves decline in cortical circuitry associated with brain-heart integration during volitional exercise. Submitted to J Neurophysiol July 23, 2016: JN-00592-2016.

Reports

1. KN Norton. Analysis of Microvasculature Modeling Parameters. Masters Independent Study; April 2008.

\section{Published Abstracts}

1. KN Norton, A Heinecke, JK Shoemaker (2015) The Neuroprotective Effects of Endurance Training on the Aging Brain. Autonomic Neuroscience: Basic and Clinical, Vol. 192, p110.

2. M Daley, KN Norton, JS Gati, JK Shoemaker. (2015) Single-subject functional parcellation of the human brainstem. Autonomic Neuroscience: Basic and Clinical, Vol. 192, p11-12.

3. KN Norton, JK Shoemaker. (2011) The hippocampal connection to the autonomic network. Autonomic Neuroscience: Basic and Clinical, Vol. 163, Issues 1-2, p50

4. R Goswami, KN Norton, MF Frances, HA Sharma, JK Shoemaker. (2011) Aging is associated with reduced white matter connectivity and fractional anisotropy in cortical autonomic regions. Autonomic Neuroscience: Basic and Clinical, Vol. 163, Issues 1-2, p50-51.

5. KN Norton, M Zamir, MF Frances, CW Usselman, A Fleischauer, JK Shoemaker. (2009) Modeling Forearm Vascular Mechanics in Hypertension. FASEB J. 23:1017.41

6. GJ Hodges, KN Norton, J Chia, KA Zuj, JK Shoemaker. (2009) Distensibility of the common carotid and carotid sinus during lower body negative pressure. FASEB J. 23:957.5.

7. AM Kiviniemi, MP Tulppo, T Seppänen, KN Norton, MF Frances, RP Nolan, HV Huikuri, JK Shoemaker. (2009) Effects of aging, hypertension, and diabetes on lowfrequency arterial pressure oscillations. FASEB J 23:1019.21.

\section{Conference Presentations}

1. KN Norton, A Heinecke, JK Shoemaker. Impact of Long-Term High Level Endurance Training on Brain Structure. Presented at The Saltin International Graduate Course in Clinical and Exercise Physiology; October 2015.

2. KN Norton, A Heinecke, R Nikolov, JK Shoemaker. Impact of Long-term High-level Endurance Training on Brain Structure in Healthy Aging. Presented at CSEP; October 2015.

3. KN Norton, TA Luchyshyn, J Drozd, R Bartha, JK Shoemaker. Impact of Long-Term Marathon Training on Age-Related Neuroanatomical Decline. Presented at ACSM; May 2015.

\section{Conference Posters}

1. KN Norton*, JK Shoemaker. Impact of age and hypertension on forebrain organization associated with heart rate regulation during exercise. Presented at the International Society for Autonomic Neuroscience; September 2011.

2. KN Norton*, TA Luchyshyn, J Drozd, R Bartha, JK Shoemaker. Impact of Long-Term Marathon Training on Age-Related Neuroanatomical Decline. Presented at CSEP; October 2014.

3. KN Norton*, R Goswami, RP Nolan, JK Shoemaker. Cortical Autonomic Alterations with Hypertension. Presented at the $21^{\text {st }}$ International Symposium on the Autonomic Nervous System; Nov 5, 2010.

4. KN Norton*, M Zamir, M Frances, CW Usselman, A Fleischhauer, JK Shoemaker. Mechanical Properties of the Forearm Vasculature in Hypertension. Presented at the Federation for American Societies of Experimental Biology; May 2009. 
5. KN Norton*, M Zamir, MF Flamengo, CW Usselman, A Fleischauer, JK Shoemaker. Mechanical Properties of the Forearm Vasculature in Hypertension. Presented at the Aging and Rehabilitation Geriatric Conference; January 2009. 